\title{
Effect of annealing on the structural and superconducting properties of $\mathrm{FeSe}_{1-x} \mathrm{Te}_{x}$
}

\author{
A dissertation submitted to the \\ Victoria University of Wellington \\ in partial fulfillment of the requirements for the degree of \\ Doctor of Philosophy \\ by \\ David Michael Uhrig \\ born on September 10th, 1989 in Malsch
}

Victoria University of Wellington

2021 



\section{Abstract}

The term 'high-temperature superconductivity' has long been synonymous with copper oxide-based superconductors (cuprates) up until the recent discovery of the iron-based superconductors in 2008. This new family of superconductors exhibits fundamentally interesting properties such as the interplay between magnetism and superconductivity as well as the very recently discovered topological properties of $\mathrm{FeSe}_{1-x} \mathrm{Te}_{x}$. Furthermore, from an application point of view, iron-based superconductors have the potential to become the new norm for low-temperature, high-field applications such as MRI and nuclear fusion. However, some of the post-processing procedures required to obtain high-quality samples, like the annealing process of $\mathrm{FeSe}_{1-x} \mathrm{Te}_{x}$, are yet to be fully understood. This thesis reports on the effect of annealing on the structure and composition of $\mathrm{FeSe}_{1-x} \mathrm{Te}_{x}$ and how they manifest as changes in the superconducting properties. Overall, air annealing is shown to improve the critical temperature and critical current density of $\mathrm{FeSe}_{1-x} \mathrm{Te}_{x}$ for almost all investigated doping concentrations. These improvements are the result of a decrease in excess iron driven by the formation of thin iron oxide layers on exposed surfaces of the crystal. Further analysis suggests that the reduction in the excess iron concentration is largest in the region right underneath the oxide layers. Consequently, the improvement in the superconducting properties is also found to be largest in these regions. In terms of the annealing atmosphere, even in nitrogen and low-vacuum atmospheres, annealing still leads to the formation of an iron oxide layer and an improvement in the superconducting properties due to the presence of residual oxygen. In rare cases, annealing was found to induce asymmetric magnetic hysteresis loops as a result of weak bulk pinning and strong surface pinning. Whilst asymmetric hysteresis loops have occasionally been reported in the cuprates and polycrystalline iron-based superconductors, this work reports the first observation of such behaviour in $\mathrm{FeSe}_{1-x} \mathrm{Te}_{x}$ single crystals. This work has deepened the understanding of the annealing process on the intrinsic properties of $\mathrm{FeSe}_{1-x} \mathrm{Te}_{x}$ and facilitates the study of additional post-processing procedures that will further improve the properties of this family of superconductors. 



\section{Acknowledgments}

I want to acknowledge and thank all people that supported me during the course of this thesis. Special thanks goes to

Shen V ChOng, probably the nicest and most caring supervisor one can have. I really appreciate the freedom and responsibility you gave me whilst still being there whenever I needed support. After more than 3 years of working together, it is hard to express my gratitude in a couple words, but I am happy I joined this project with you as my supervisor.

Grant V M WiLliams for sharing his knowledge and the crucial feedback during discussions. Besides the professional efforts, you and Shen have always made sure that things are not only going well at work but also outside of the direct work environment. Starting all over in a new country is never easy, but you made sure that my start would be as smooth and easy as possible.

GABRiEl Bioletti for being my first point of contact to test any of my crazy ideas. It has been a pleasure working with you.

SARAH SPEnCER for the uplifting attitude and the performance of all SEM measurements presented in this thesis.

The WhOlE STAFF of the Robinson Research Institute for always being supportive and uplifting. In this context, I want to especially thank JEANNIE REDMAN and URsula MuavaE for always having their door open and making sure that I have everything I need.

$\mathrm{Zu}$ guter Letzt möchte ich mich bei meinen Eltern bedanken, die mich bei allem unterstützen was ich mir in meinen Kopf setze... auch wenn ich dazu zum anderen Ende der Welt reisen muss. 



\section{Contents}

$\begin{array}{lll}1 & \text { Introduction } & 1\end{array}$

\begin{tabular}{|lll}
\hline 2 & Theoretical background & 5
\end{tabular}

2.1 Introduction to superconductivity . . . . . . . . . . . . . 5

2.1 .1 Zero electrical resistance $\ldots \ldots \ldots \ldots \ldots$

2.1 .2 Meissner-Ochsenfeld effect . . . . . . . . . . . . . 7

2.1 .3 London equations $\ldots \ldots \ldots \ldots$

2.1 .4 Thermodynamic description . . . . . . . . . . . . . . 11

2.1 .5 Type I and type II superconductor. . . . . . . . . . . . . . . . . 12

2.1 .6 Abrikosov lattice . . . . . . . . . . . . . . . . . . . . . . 15

2.1.7 Vortex dynamics and critical current density in type II superconductors 16

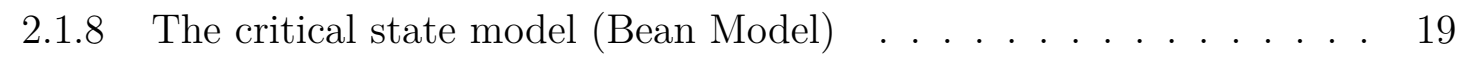

$2.1 .9 \quad$ Anderson flux creep $\ldots \ldots \ldots$. . . . . . . . . . . . . . . . . . . . 24

2.1 .10 Sweep-rate-dependence of MHLs . . . . . . . . . . . . . . . 27

2.2 Iron-based superconductors . . . . . . . . . . . . . . . . . . . . . 28

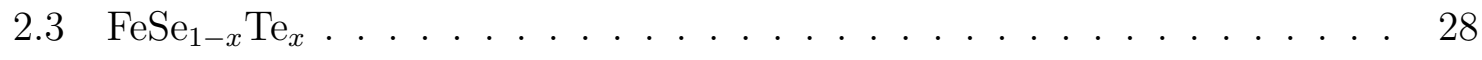

$2.3 .1 \quad$ Crystal structure and excess iron . . . . . . . . . . . . . . . . 29

$2.3 .2 \quad$ Superconducting phase diagram . . . . . . . . . . . . . 30

2.3 .3 Annealing $\ldots \ldots \ldots \ldots . \ldots \ldots$ 
\begin{tabular}{|lll}
\hline 3 & Experimental methods & 35
\end{tabular}

$3.1 \quad$ Sample synthesis $\ldots \ldots \ldots \ldots$. . . . . . . . . . . . . . . . . . . . . . 35

3.2 Annealing $\ldots \ldots \ldots \ldots \ldots$

$3.3 \quad$ Vibrating sample magnetometer $(\mathrm{VSM}) \ldots \ldots \ldots \ldots$

3.4 Magnetic characterisation measurements . . . . . . . . . . . . . . 43

$3.4 .1 \quad$ Zero-field-cooled and field-cooled magnetisation . . . . . . . . . 43

3.4 .2 Magnetic hysteresis loop measurements . . . . . . . . . . . . . . 47

3.5 Raman spectroscopy $\ldots \ldots \ldots \ldots$. . . . . . . . . . . . . . . . . 48

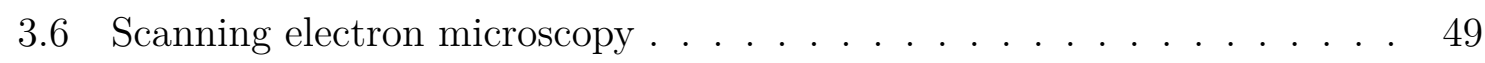

3.6.1 $\quad$ Backscattered electron detection (BSE) . . . . . . . . . . . . . . . 49

$3.6 .2 \quad$ Energy-dispersive X-ray spectroscopy (EDS) . . . . . . . . . . . . . 50

3.6.3 $\quad$ Scanning transmission electron microscopy (STEM) . . . . . . . . . 50

3.7 Depth-profiling via X-ray photoelectron spectroscopy . . . . . . . . . . 51

3.8 X-ray Diffractometry $\ldots \ldots \ldots \ldots \ldots \ldots$. . . . . . . . . . . . . . 52

4 Effect of air annealing on the structure and composition of

$\begin{array}{lc}\mathrm{FeSe}_{1-x} \mathrm{Te}_{x} & 53\end{array}$

4.1 XRD characterisation of as-grown $\mathrm{FeSe}_{1-x} \mathrm{Te}_{x} \quad \ldots \ldots \ldots$. . . . . . 53

4.2 Optical microscopy and SEM-EDS . . . . . . . . . . . . . . . . . . 58

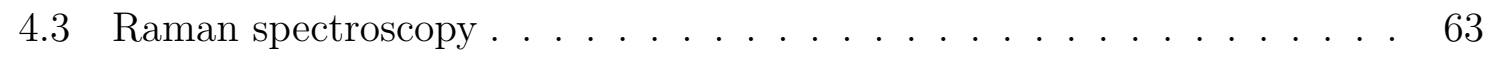

4.4 Cross-sectional Raman spectroscopy and SEM analysis . . . . . . . . . 66

4.4 .1 Raman spectroscopy $\ldots \ldots \ldots$. . . . . . . . . . . . . . 66

$4.4 .2 \quad$ SEM analysis $\ldots \ldots \ldots \ldots$. . . . . . . . . . . . . . . . . . . . .

4.5 Cross-sectional STEM analysis . . . . . . . . . . . . . . . . . 71 
$4.6 \quad$ Depth-profiling X-Ray photoelectron spectroscopy (XPS) analysis . . . 75

4.7 Theoretical considerations on the iron oxide layer thickness . . . . . . . 85

4.7 .1 Constructive surface-interface interference . . . . . . . . . . . . . . 85

4.7.2 Estimate of the iron oxide thickness for complete removal of the interstitial excess iron . . . . . . . . . . . . . . . . . . . . . . 88

4.8 Diffusion model $\ldots \ldots \ldots$. . . . . . . . . . . . . . . . . . . . . . . . . 90

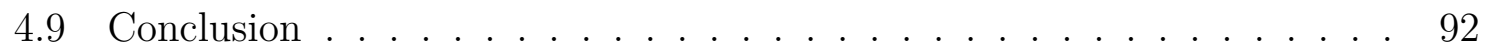

5 Effect of air annealing on the superconducting properties of

$\mathbf{F e S e}_{1-x} \mathbf{T e}_{x}$

$5.1 \quad$ ZFC-FC magnetisation of as-grown and air-annealed $\mathrm{FeSe}_{1-x} \mathrm{Te}_{x} \ldots \ldots$

$5.2 \quad$ MHLs and $J_{c}$ of as-grown and air-annealed $\mathrm{FeSe}_{1-x} \mathrm{Te}_{x} \ldots \ldots$. . . . . . 99

5.3 Thickness-dependent superconducting properties of $\mathrm{FeSe}_{0.35} \mathrm{Te}_{0.65}$. . . . 104

$5.3 .1 \quad$ Optical microscopy $\ldots \ldots \ldots$. . . . . . . . . . . . . . 104

$5.3 .2 \quad$ ZFC-FC susceptibility . . . . . . . . . . . . . 106

$5.3 .3 \quad$ Magnetic hysteresis loops . . . . . . . . . . . . . . . . . . 109

5.3 .4 Critical Current Density $\ldots \ldots \ldots$. . . . . . . . . . 117

5.4 Conclusion . . . . . . . . . . . . . . . . . . . . . . 118

6 Effect of annealing in inert atmospheres on $\mathrm{FeSe}_{1-x} \mathrm{Te}_{x} \quad 121$

$6.1 \quad$ Emergence of a universal superconducting phase diagram . . . . . . . . 122

6.2 Raman analysis of as-grown and annealed $\mathrm{FeSe}_{0.35} \mathrm{Te}_{0.65}$. . . . . . . . . 124

$6.3 \quad$ ZFC-FC susceptibility of as-grown and annealed $\mathrm{FeSe}_{0.35} \mathrm{Te}_{0.65}$. . . . . 125

6.4 Critical current density of as-grown and annealed $\mathrm{FeSe}_{0.35} \mathrm{Te}_{0.65}$. . . . . 126

6.5 Summary . . . . . . . . . . . . . . . . . . . . . . 127 
6.6 Estimation of residual oxygen present in different annealing atmospheres 128

6.7 Conclusion . . . . . . . . . . . . . . . . . . . . . . . 132

7 Asymmetric magnetic hysteresis loops in $\mathrm{FeSe}_{1-x} \mathrm{Te}_{x}$

$7.1 \quad$ Asymmetric magnetic hysteresis loops . . . . . . . . . . . . . . . . . . . 134

7.2 Instability of the asymmetric MHL state $\ldots \ldots \ldots$. . . . . . . . . 137

7.3 Sweep-rate-dependent MHLs . . . . . . . . . . . . . . . . . . . . . . . . 139

7.4 Time-dependent magnetic relaxation . . . . . . . . . . . . . . . . 141

7.5 Sweep-rate-dependent magnetic relaxation . . . . . . . . . . . . . . . . 148

$7.6 \quad$ Pinning model for the asymmetric MHL and relaxation . . . . . . . . . 154

7.7 Asymmetric hysteresis loops in the literature . . . . . . . . . . . . . . . 160

7.7.1 Asymmetric MHLs in polycrystalline samples . . . . . . . . . . . . . 161

7.7.2 Interaction between ferromagnetism and superconductivity . . . . . 162

7.8 Conclusion $\ldots \ldots \ldots \ldots$

\begin{tabular}{lll}
\hline 8 & Conclusion & 165
\end{tabular}

\begin{tabular}{lr}
\hline Publications & 169
\end{tabular}

\begin{tabular}{lr}
\hline Bibliography & 171
\end{tabular} 


\section{Introduction}

The observation of superconductivity in the $\mathrm{BaLaCuO}$ system with a reported superconducting transition temperature of $T_{\mathrm{c}}=30 \mathrm{~K}$ Bed86] dawns the age of high-temperature superconductivity and was quickly followed by the discovery of more copper-oxide based superconductor (cuprates) such as $\mathrm{YBa}_{2} \mathrm{Cu}_{3} \mathrm{O}_{7-x}$ [Wu87, Hik87, Tin96] and $\mathrm{BiSrCaCu}_{2} \mathrm{O}_{x}$ Mae88, with $T_{\mathrm{c}}=93 \mathrm{~K}$ and $T_{\mathrm{c}}=105 \mathrm{~K}$, respectively. With $T_{\mathrm{c}}$ above the boiling point of liquid nitrogen, high critical current densities, $J_{\mathrm{c}}$, and high upper critical fields, $H_{\mathrm{c} 2}$, cuprates instantly created high hopes for the application of superconductors in high-field magnets and superconducting devices [Dew01, Mal05]. As it turned out, those high expectations were met with disappointment due to the complex synthesis procedures of this new class of superconductors and challenging material properties such as highly resistive grain boundaries and high magnetic anisotropy Mal05]. Even 40 years after their initial discovery, cuprates are still extensively researched to overcome some of the barriers for application and commercial wire and devices have only recently become available Mal05.

For a long time, high-temperature superconductivity was thought to be confined to the cuprates. This changed with the discovery of superconductivity in the ironbased compound $\mathrm{LaFeAsO}_{1-\mathrm{x}} \mathrm{F}_{\mathrm{x}}$ approximately 13 years ago [Kam08a. This initial observation sparked interest in the search for other iron-based superconductors and was rapidly followed by the discovery of other superconducting compounds such as $\mathrm{Ba}_{1-\mathrm{x}} \mathrm{K}_{\mathrm{x}} \mathrm{Fe}_{2} \mathrm{As}_{2}$ [Rot08], LiFeAs Wan08, and FeSe [Hsu08]. Similar to the cuprates, the family of iron-based superconductors also shows promising properties for application such as relatively high $T_{\mathrm{c}}$, large $H_{\mathrm{c} 2}$ and $J_{\mathrm{c}}$, and, most of all, reduced anisotropy in comparison to the cuprates [Put10, Shi14, Pal15]. These properties make the ironbased superconductors an ideal candidate for the application in environments that 
require large magnetic fields such as fusion, NMR, MRI, or MAGLEV trains Shi14. However, given the relatively recent discovery, there are still obstacles that need be overcome to reach their full potential for practical application [Put10, Shi14, Pal15].

This thesis focuses on the tellurium-doped iron selenide family of superconductors $\left(\mathrm{FeSe}_{1-x} \mathrm{Te}_{x}\right)$, which represents the structurally simplest form of iron-based superconductors. Since the discovery of superconductivity in the parent compound by $\mathrm{Hsu}$ et al. in 2008 [Hsu08, the $\mathrm{FeSe}_{1-x} \mathrm{Te}_{x}$ family of superconductors has attracted much attention and has been featured in more than 15000 publications. This is largely due to the relative ease of synthesising large single crystals and the emergence of exotic properties, such as, a highly tuneable superconducting transition temperature by ionic liquid gating [Lei16, Kou18] or under hydrostatic pressure [Med09], a very high $T_{\mathrm{c}}$ for single-layer FeSe [Ge14] and, more recently, the discovery of topological properties making this compound the first high-temperature topological superconductor [Zha18, Zha19]. However, the synthesis of $\mathrm{FeSe}_{1-x} \mathrm{Te}_{x}$ invariably leads to the presence of magnetic interstitial excess iron [Bao09, McQ09, Zha09], which has been shown to suppress superconductivity in this family of superconductors [McQ09, Zha09, Lei10, Liu09. As a result, samples of varying quality have led to many properties such as their doping-dependent superconducting phase diagram still being controversial and not fully established even though $\mathrm{FeSe}_{1-\mathrm{x}} \mathrm{Te}_{\mathrm{x}}$ has been subject to extensive research [Sal09, Tae09, Miz10, Bao09, Don11, Kaw12, Sun16, Noj10, Tam16]. In order to further advance the understanding of this family of superconductors and its applications, it is essential to establish reproducible growth and post-processing procedures that produce high-quality samples.

The aim of this thesis is to help fill this knowledge-gap on $\mathrm{FeSe}_{1-x} \mathrm{Te}_{x}$ with a focus on the effect of annealing as a mean of post-processing. The first three result chapters concern the structure of differently doped $\mathrm{FeSe}_{1-x} \mathrm{Te}_{x}$ single crystals before and after annealing in different annealing atmospheres and connect the findings to changes in the superconducting properties. In this context, this thesis tries to answer 
three questions. First, what happens during annealing and how does it lead to an improvement in the superconducting properties? Secondly, how are the changes in structure and consequently the superconducting properties related to the annealing atmospheres? And lastly, are the induced changes limited to the surface of the single crystals or is annealing effective in improving the properties of the whole crystal?

The thesis is structured as follows:

In chapter 2, a general introduction to superconductivity and iron-based superconductors is given. This introduction starts with basic concepts such as the identification of a superconductor based on the zero-resistance- and Meissner-Ochsenfeld state, the introduction of characteristic parameters like the London penetration depth and the Ginzburg-Landau coherence length, and the classification of type I and type II superconductors. Later in the chapter, topic-specific concepts that are relevant for the understanding of this thesis such as the Bean model and Anderson flux creep are introduced. Lastly, a short overview on the recently discovered iron-based superconductors and $\mathrm{FeSe}_{1-x} \mathrm{Te}_{x}$ with a focus on the properties that are relevant to this thesis is given.

Chapter 3 summarises the experimental methods used in this work, the procedure for the synthesis of the single crystals, and basic XRD characterisation. Given the importance of magnetic measurements to this thesis, this chapter also provides a detailed introduction of the working principle of a vibrating sample magnetometer (VSM) and the different measurement modes that were used in this work. Additionally, a brief introduction to scanning electron microscopy (SEM), Raman spectroscopy, and $\mathrm{X}$-ray photoelectron spectroscopy (XPS) is given.

Chapter 4 presents a comprehensive analysis of the structural changes induced in $\mathrm{FeSe}_{1-x} \mathrm{Te}_{x}$ after annealing in air. In the first part, the analysis of the surface of asgrown and air-annealed single crystals using optical microscopy, Raman spectroscopy, and SEM-EDS is presented. After that, the cross sections of two $\mathrm{FeSe}_{0.35} \mathrm{Te}_{0.65}$ single crystals are investigated using Raman spectroscopy, SEM, and STEM analysis. The 
findings from the SEM and STEM analysis of the cross sections are then compared to the findings from depth-profiling X-Ray photoelectron spectroscopy of an as-grown and air-annealed single crystal. To put the experimental results in context, theoretical considerations on the oxidation process are presented. Lastly, the observed oxidation process during annealing is explained based on a proposed diffusion model.

In chapter 5, the effect of air annealing on the superconducting properties of $\mathrm{FeSe}_{1-x} \mathrm{Te}_{x}$ is studied. First, ZFC-FC-magnetisation and magnetic hysteresis loop measurements of four differently doped single crystals are presented. The observed changes in the superconducting properties are then linked to the structural changes observed in chapter 4. In the last part of this chapter, the thickness-dependence of the annealing process is studied by analysing the magnetic properties of a stepwise exfoliated and air-annealed single crystal.

Chapter 6 concerns the effect of annealing in inert atmospheres on the structural and magnetic properties of $\mathrm{FeSe}_{0.35} \mathrm{Te}_{0.65}$. In the first part of this chapter, the superconducting phase diagram of as-grown and annealed $\mathrm{FeSe}_{1-x} \mathrm{Te}_{x}$ based on the literature and this work is presented and analysed. Afterwards, the effect of annealing in air, nitrogen, low-vacuum, and high-vacuum are studied using Raman spectroscopy and magnetisation measurements. Lastly, the experimental results are explained based on theoretical estimates of the residual oxygen concentration that is expected to be present in the different annealing atmospheres.

The last chapter, chapter 7, investigates the asymmetric magnetic hysteresis loops that were observed in some samples. First, the nature of the asymmetric hysteresis loop is introduced and shown to be an intrinsic property of the samples and not the result of external distortions or malfunctions. After this, the pinning behaviour is studied by means of time-dependent and sweep-rate-dependent magnetic relaxation measurements. This is followed by the proposal of a pinning model that combines both bulk and surface pinning to explain the observed experimental results. Lastly, the results are put in context to similar results that were observed in the cuprates and some polycrystalline iron-based superconductors. 


\section{Theoretical background}

This chapter introduces the basic concepts of superconductivity and the theoretical background necessary for the understanding of this thesis. First, a short general introduction on superconductivity, its basic phenomena, and the characteristic parameters is given. In the second part of this chapter, a literature review on the iron-based superconductors and in particular the $\mathrm{FeSe}_{1-x} \mathrm{Te}_{x}$ family of superconductors is presented.

\subsection{Introduction to superconductivity}

The development of the theory of superconductivity was initiated by its discovery in 1911 [Kam11] and is still ongoing. As such, a complete summary of the current state of the theory would extend beyond the scope of this thesis. Instead, the aim of the first part of this chapter is to give a brief introduction to the key phenomena and parameter of superconductivity with a focus on the phenomenological theory of superconductivity. For a more detailed deduction and an introduction to the microscopic BCS-theory [Bar57], the reader is directed to the textbooks by Tinkham [Tin96] and Enss et al. [Ens05].

\subsubsection{Zero electrical resistance}

The two key properties that define a superconductor are zero electrical resistance and perfect diamagnetism (also known as the Meissner-Ochsenfeld effect). The former was first observed by Sir Kamerlingh Onnes in Leiden in 1911 for the case of mercury Kam11]. When he cooled mercury down below a certain temperature, the resistance 
rapidly dropped below the lowest measurable resistance of his experimental setup 1 . This drop in resistivity to zero at a certain temperature is universally observed for all superconducting materials and the temperature at which the drop occurs is defined as the critical temperature, $T_{\mathrm{c}}$.

Table 2.1 shows a summary of the critical temperatures of the elemental superconductors, selected compound superconductors, and several high-temperature superconductors [Ens05, Bed86, Wu87, Mae88, Kam08, Rot08, Hsu08]. As it turns out, about half of all stable elements become superconducting below their respective critical temperature. Interestingly however, some of the best elemental room temperature conductors, such as copper, silver, and gold, have not yet been found to turn

Table 2.1: Critical temperatures of the elemental superconductors, selected compound superconductors, and several high-temperature superconductors under atmospheric pressure [Ens05, Bed86, Wu87, Mae88, Kam08, Rot08, Hsu08.

\begin{tabular}{ll|ll|ll} 
Element & $T_{\mathrm{c}}(\mathrm{K})$ & Element & $T_{\mathrm{c}}(\mathrm{K})$ & Element & $T_{\mathrm{c}}(\mathrm{K})$ \\
\hline $\mathrm{Al}$ & 1.19 & $\mathrm{Nb}$ & 9.2 & $T_{\mathrm{c}}$ & 7.8 \\
$\mathrm{Be}$ & 0.026 & $\mathrm{~Np}$ & 0.075 & $\mathrm{Th}$ & 1.37 \\
$\mathrm{Cd}$ & 0.55 & $\mathrm{Os}$ & 0.65 & $\mathrm{Ti}$ & 0.39 \\
$\mathrm{Ga}$ & 1.09 & $\mathrm{~Pa}$ & 1.3 & $\mathrm{Tl}$ & 2.39 \\
$\mathrm{Hf}$ & 0.13 & $\mathrm{~Pb}$ & 7.2 & $\mathrm{U}$ & 0.2 \\
$\mathrm{Hg}$ & 4.15 & $\mathrm{Re}$ & 1.7 & $\mathrm{~V}$ & 5.3 \\
$\mathrm{In}$ & 3.40 & $\mathrm{Rh}$ & 0.0003 & $\mathrm{~W}$ & 0.012 \\
$\mathrm{Ir}$ & 0.14 & $\mathrm{Ru}$ & 0.5 & $\mathrm{Zn}$ & 0.9 \\
$\mathrm{La}$ & 4.8 & $\mathrm{Sn}$ & 3.75 & $\mathrm{Zr}$ & 0.55 \\
$\mathrm{Mo}$ & 0.92 & $\mathrm{Ta}$ & 4.39 & &
\end{tabular}

\begin{tabular}{ll|ll|ll} 
Compound & $T_{\mathrm{c}}(\mathrm{K})$ & Compound & $T_{\mathrm{c}}(\mathrm{K})$ & Compound & $T_{\mathrm{c}}(\mathrm{K})$ \\
\hline $\mathrm{Nb}_{3} \mathrm{Sn}$ & 18.1 & $\mathrm{Nb}_{3} \mathrm{Ge}$ & 23.2 & $\mathrm{MgB}_{2}$ & 39
\end{tabular}

\begin{tabular}{ll|ll} 
High- $T_{\mathrm{c}}($ Cuprates $)$ & $T_{\mathrm{c}}(\mathrm{K})$ & High- $T_{\mathrm{c}}($ Iron-based $)$ & $T_{\mathrm{c}}(\mathrm{K})$ \\
\hline $\mathrm{Ba}_{x} \mathrm{La}_{5-x} \mathrm{Cu}_{5} \mathrm{O}_{5}$ & 30 & $\mathrm{La}\left(\mathrm{O}_{1-x} \mathrm{~F}_{x}\right) \mathrm{FeAs}$ & 26 \\
$\left(\mathrm{Y}_{0.6} \mathrm{Ba}_{0.4}\right)_{2} \mathrm{CuO}_{4-\delta}$ & 93 & $\mathrm{Ba}_{1-x} \mathrm{~K}_{x} \mathrm{Fe}_{2} \mathrm{As}_{2}$ & 38 \\
$\mathrm{BiSrCaCu}_{2} \mathrm{O}_{x}$ & 105 & FeSe & 8
\end{tabular}

\footnotetext{
${ }^{1}$ Later persistent current experiments have shown that the currents would persist for more than $10^{5}$ years indicating an actual zero resistivity state Qui62, Ens05, Tin96.
} 
superconducting. Overall, the critical temperature of all elemental superconductors is very low with $\mathrm{Nb}$ having the highest $T_{\mathrm{c}}$ of $9.2 \mathrm{~K}$.

Compound superconductors exhibit higher critical temperatures of up to $39 \mathrm{~K}$ as in the case of $\mathrm{MgB}_{2}$, but the real revolution came with the discovery of the so-called high-temperature superconductors. As hinted at by their name, these compounds turn superconducting at 'high' temperatures with some having critical temperatures above $100 \mathrm{~K}$, which is above the boiling temperature of liquid nitrogen $(77 \mathrm{~K})$ [Dew01, Ens05, Tin96]. To this day, there are two main groups of high-temperature superconductors: the cuprates and the iron-based superconductors. The former were discovered in 1986 by Bednorz and Mueller and are based on copper oxide compounds [Bed86]. The latter, which are the focus of this thesis, were only recently discovered by the Hosono Group in 2006 [Kam06, Kam08a] and have since been puzzling the scientific community as they combine superconductivity and magnetism, which before have been thought to exclude each other [Joh10].

\subsubsection{Meissner-Ochsenfeld effect}

The second key property of a superconductor is the Meissner-Ochsenfeld effect, which distinguishes a superconductor from a mere ideal conductor (zero electrical resistance). The difference between a superconductor and a conceptional ideal conductor is typically illustrated by the thought experiment depicted in figure 2.1. First, a small magnetic field is applied at a temperature above the critical temperature. At this point, the magnetic field penetrates both the ideal conductor and the superconductor equally as they are still in a normal conducting state. Eddy currents are induced in both to oppose the change in flux [Gri08], but because of the electrical resistance, they eventually decay.

When the ideal conductor is cooled below the critical temperature, the magnetic fields continues to penetrate the sample. On the contrary, the superconductor fully expels the magnetic field acting as a perfect diamagnet. This unexpected expulsion 


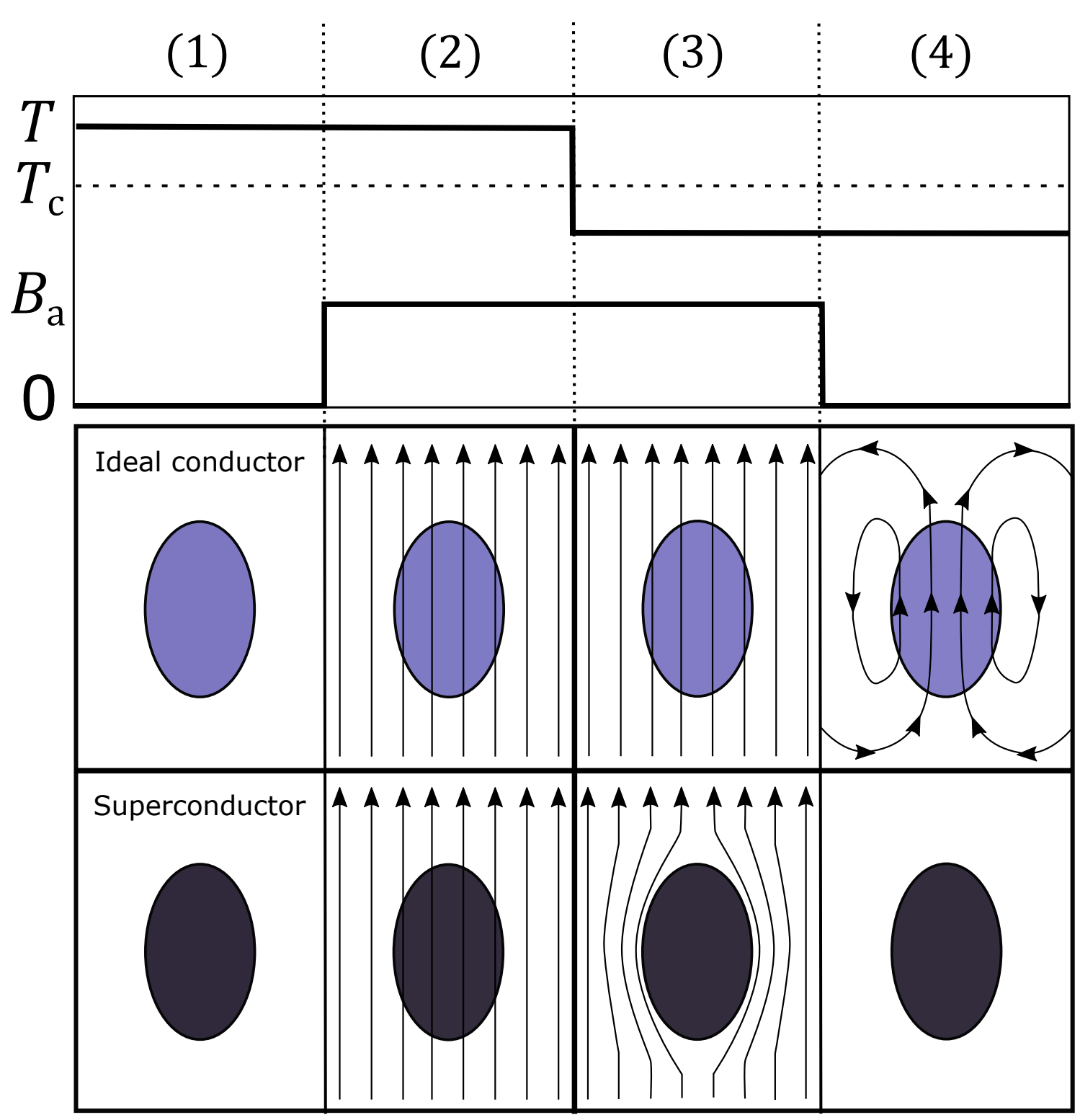

Figure 2.1: Schematic of a thought experiment on the behaviour of a superconductor versus an ideal conductor for a specific cooling and applied magnetic field, $B_{\mathrm{a}}$, cycle. This schematic has been adapted from [Ens05].

of small external magnetic fields was discovered by Meissner and Ochsenfeld in 1933 Mei33 and is therefore commonly referred to as the Meissner-Ochsenfeld effect. As was shown later [Bar57, Tin96, Ens05, Dew01, this effect is a consequence of the superconducting state: the energy cost of expelling the magnetic field is smaller than the energy gain due to the condensation in the superconducting state.

The differences between an ideal conductor and a superconductor are further illus- 
trated in the last step, where the applied magnetic field is turned back to zero. In the case of the ideal conductor, the change in the magnetic flux density induces screening currents that act against the change according to Faraday's law Gri08]. Because the ideal conductor has no electrical resistance, the screening currents conserve the flux in the sample indefinitely. The superconductor, on the other hand, was not penetrated by magnetic flux in the first place due to its perfect diamagnetism. Thus, no change in flux occurs and no screening currents are induced. The whole cycle is reversible as a result of the superconducting phase transition [Tin96, Ens05, Dew01]. As stated by Dew-Hughes, "the importance of this discovery of the Meissner effect to the understanding of superconductivity cannot be overemphasised" Dew01]. It is the key indicator that differentiates between an ideal conductor and an actual superconductor, and enabled the development of a thermodynamic description of the superconducting state [Gin50, Tin96, Ens05, Dew01].

\subsubsection{London equations}

One of the first electromagnetic descriptions of superconductivity was introduced by the London brothers, Heinz and Fritz London, in 1935 [Lon35]. Their model not only inherited the two key features of zero resistance and perfect diamagnetism, but also predicted the exponential decay of external magnetic fields in a thin surface sheath described by the London penetration depth, $\lambda_{\mathrm{L}}$. The first London equation can be derived from the Drude model of conductivity by setting the collision time of the charge carrier to $\tau=\infty 2$. The resulting equation describes perfect conductivity through the relation between the electric field, $\boldsymbol{E}$, and the change in current density, $\boldsymbol{J}$, with time:

$$
\boldsymbol{E}=\frac{\partial}{\partial t}\left(\frac{m}{n_{\mathrm{s}} e^{2}} \boldsymbol{J}\right)=\frac{\partial}{\partial t}(\Lambda \boldsymbol{J})
$$

where $m$ is the mass of the charge carrier, $e$ the elemental charge, $n_{s}$ the superconducting charge carrier density, and $\Lambda=m / n_{\mathrm{s}} e^{2}$, a phenomenological parameter.

\footnotetext{
${ }^{2}$ Technically, this derivation is not rigorous [Tin96, Ens05]. However, it does convey the relevant ideas necessary for the understanding of this thesis.
} 
This equation is denoted as 'acceleration equation' in the original London paper as it describes the proportionality of the electric field to the change in the critical current density rather than the critical current density itself as in the case of normal conductors (Ohm's law) [Lon35, Ens05, Tin96]. From this, it follows that stationary, persistent currents can run in a superconductor without the presence of an electric field.

The second London equation relates the magnetic field, $\boldsymbol{B}$, to the curl of the current density

$$
\boldsymbol{B}=-\Lambda(\nabla \times \boldsymbol{J})
$$

When inserting the second Maxwell equation, $\nabla \times \boldsymbol{B}=\mu_{0} \boldsymbol{J}$, into equation 2.2 one obtains

$$
\begin{aligned}
\boldsymbol{B} & =-\Lambda\left(\nabla \times\left(\frac{\nabla \times \boldsymbol{B}}{\mu_{0}}\right)\right) \\
& =-\frac{\Lambda}{\mu_{0}}(\nabla \times(\nabla \times \boldsymbol{B})) \\
& =-\frac{\Lambda}{\mu_{0}}\left(\nabla(\nabla \cdot \boldsymbol{B})-\nabla^{2} \boldsymbol{B}\right) .
\end{aligned}
$$

Since $\nabla \cdot \boldsymbol{B}=0$ (Maxwell equation, no magnetic monopoles) Gri08, equation 2.3 can further be simplified and rearranged to

$$
\nabla^{2} \boldsymbol{B}=\frac{\mu_{0}}{\Lambda} \boldsymbol{B}=\frac{\boldsymbol{B}}{\lambda_{\mathrm{L}}^{2}}
$$

where $\lambda_{\mathrm{L}}$ is the London penetration depth. For the case of a superconductor occupying the half space for $x>0$ and vacuum for $x<0$ and a magnetic field applied along the $z$-direction (see figure 2.2, this Poisson-like equation can be simplified and rearranged to the one-dimensional Poisson equation

$$
\frac{\partial^{2}}{\partial x^{2}} B_{z}-\frac{B_{z}}{\lambda_{\mathrm{L}}^{2}}=0
$$




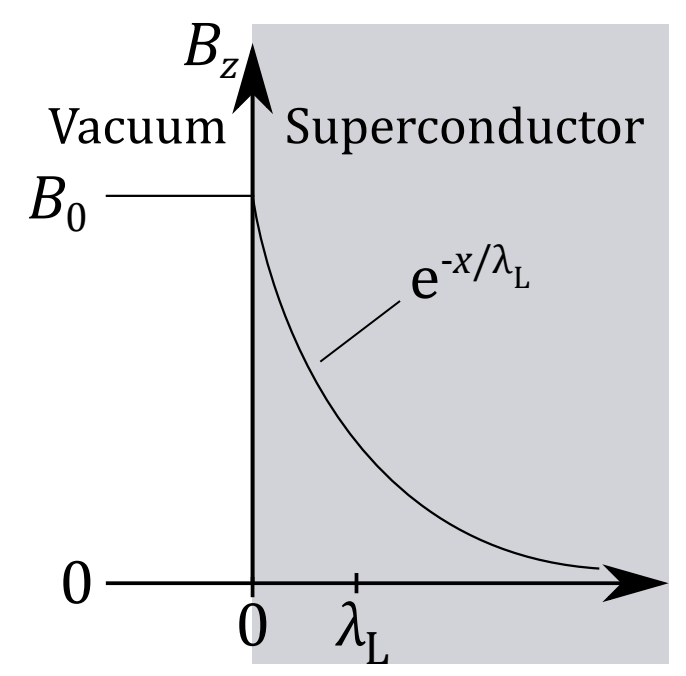

Figure 2.2: Magnetic field profile inside a superconductor as described by the London model. This schematic has been adapted from [Ens05].

which is solved by the ansatz

$$
B_{z}=B_{0} \mathrm{e}^{-x / \lambda_{\mathrm{L}}}
$$

This exponential decay of the magnetic field can further be extended to the exponential decay of the critical current density via the aforementioned Maxwell relation $\nabla \times \boldsymbol{B}=\mu_{0} \boldsymbol{J}$ Gri08]. For the case of the half-space superconductor described above, this leads to

$$
J_{y}=\frac{B_{0}}{\lambda_{\mathrm{L}}} \mathrm{e}^{-x / \lambda_{\mathrm{L}}}
$$

Therefore, both the magnetic field and the critical current decay exponentially from the interface over the characteristic length scale of the London penetration depth. Figure 2.2 depicts the exponential decay of the magnetic field inside a superconductor following equation 2.6.

\subsubsection{Thermodynamic description}

In 1950, Ginzburg and Landau introduced the idea of a complex pseudo-wavefunction $\psi$ as an order parameter to describe the superconducting state within Landau's the- 
ory of second-order phase transitions and relate the order parameter to the superconducting carrier density:

$$
n_{\mathrm{s}}=|\psi(x)|^{2}
$$

[Gin50, Tin96]. In the context of this theory, the characteristic distance over which the wave function can transition from the superconducting state to the normal state, the coherence length $\$^{3}$, is introduced. This parameter is important as it relates to how quickly (spatially) a superconductor can change between the normal- and superconducting state. As will be shown later, depending on the value of $\xi$ and $\lambda_{\mathrm{L}}$, a superconductor behaves very differently.

\subsubsection{Type I and type II superconductor}

Based on Ginzburg-Landau (GL) theory, it can be shown that there are two types of superconductor, type I and type II [Abr57, Abr73, Ens05, Tin96]. As was first shown by Abrikosov, this categorisation results from the interface energy between the superconducting and non-superconducting phase, which is either positive or negative depending on the ratio of the penetration depth to the coherence length. This ratio is typically referred to as the Ginzburg-Landau parameter

$$
\kappa=\frac{\lambda}{\xi}
$$

Depending on the value of $\kappa$ and the resulting sign of the interface energy, a superconductor is either considered a type I or type II superconductor:

$$
\begin{aligned}
& \kappa>\frac{1}{\sqrt{2}}-\text { Type I } \\
& \kappa<\frac{1}{\sqrt{2}}-\text { Type II . }
\end{aligned}
$$

\footnotetext{
${ }^{3}$ It is worth mentioning that a similar concept of the coherence length was also independently introduced by Pippard Pip53. However, in this thesis, the coherence length always refers to the GL-coherence length.
} 

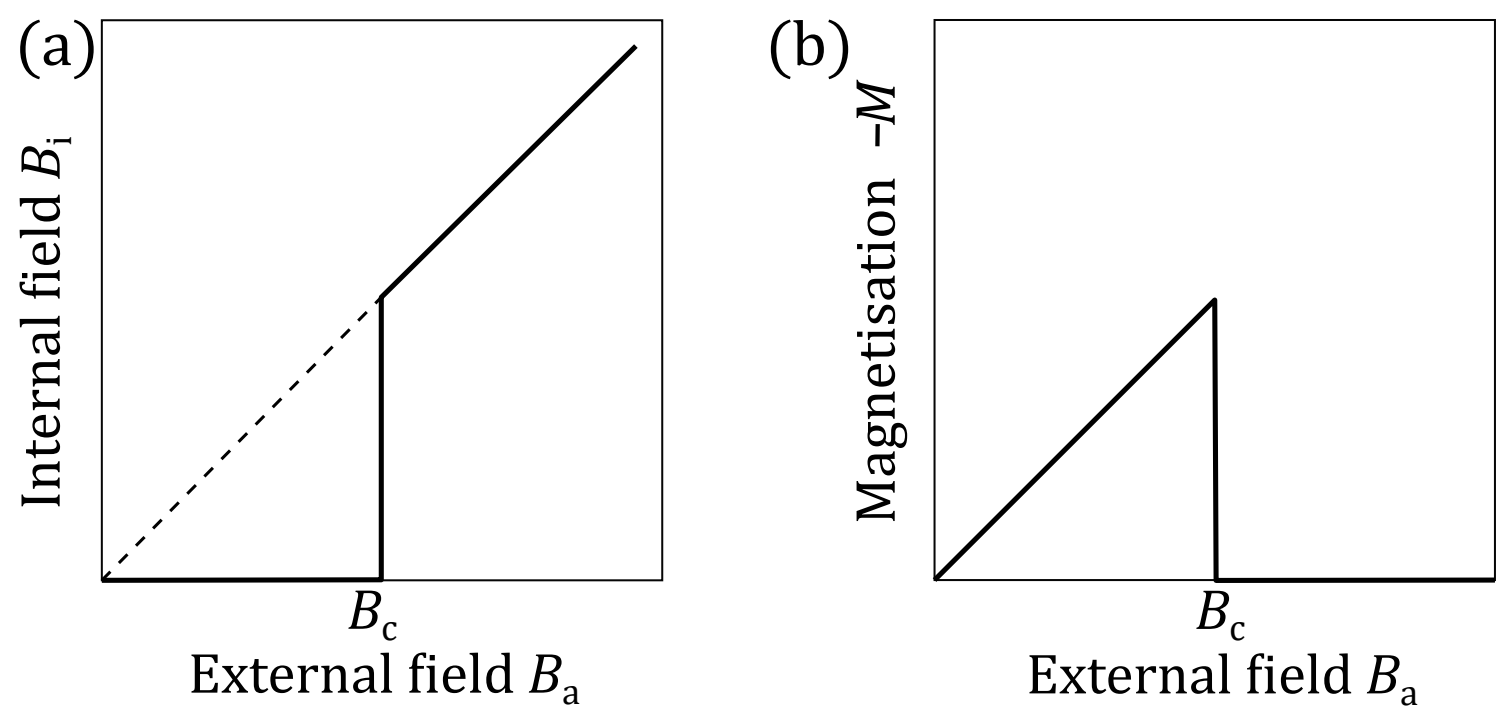

Figure 2.3: (a) Internal field, $B_{\mathrm{i}}$, and (b) negative magnetisation, $-M$, versus applied external field $B_{\mathrm{a}}$ for an ideal type I superconductor without demagnetisation. At first, the magnetic field is expelled until the critical field, $B_{\mathrm{c}}$, is reached. At fields above $B_{\mathrm{c}}$, superconductivity is lost and the magnetic field penetrates the superconductor.

As will be shown in the following sections, type I and type II superconductors exhibit severe differences in their superconducting and magnetic properties.

\section{Type I superconductors}

In a type I superconductor, the penetration depth is smaller than the coherence length and an energy penalty is associated with superconducting/nonsuperconducting interfaces. As a consequence, the magnetic field is expelled until a certain threshold value $\left(B_{\mathrm{c}}\right)$, at which the energy necessary to expel the magnetic field exceeds the energy gain of the superconducting state, is reached 4 The superconductor acts as a perfect diamagnet and exhibits the Meissner-Ochsenfeld effect until the applied field exceeds $B_{\mathrm{c}}$ and superconductivity is suppressed. The resulting internal field and magnetisation profile versus the external field of an ideal type I superconductor are depicted in figure 2.3 .

Most elemental superconductors are of type I and it was long believed that there are

\footnotetext{
${ }^{4}$ It should be noted that due to demagnetisation effects, an intermediate state is observed for non-ellipsoidal samples. More on this can be found in [Ens05, Tin96].
} 

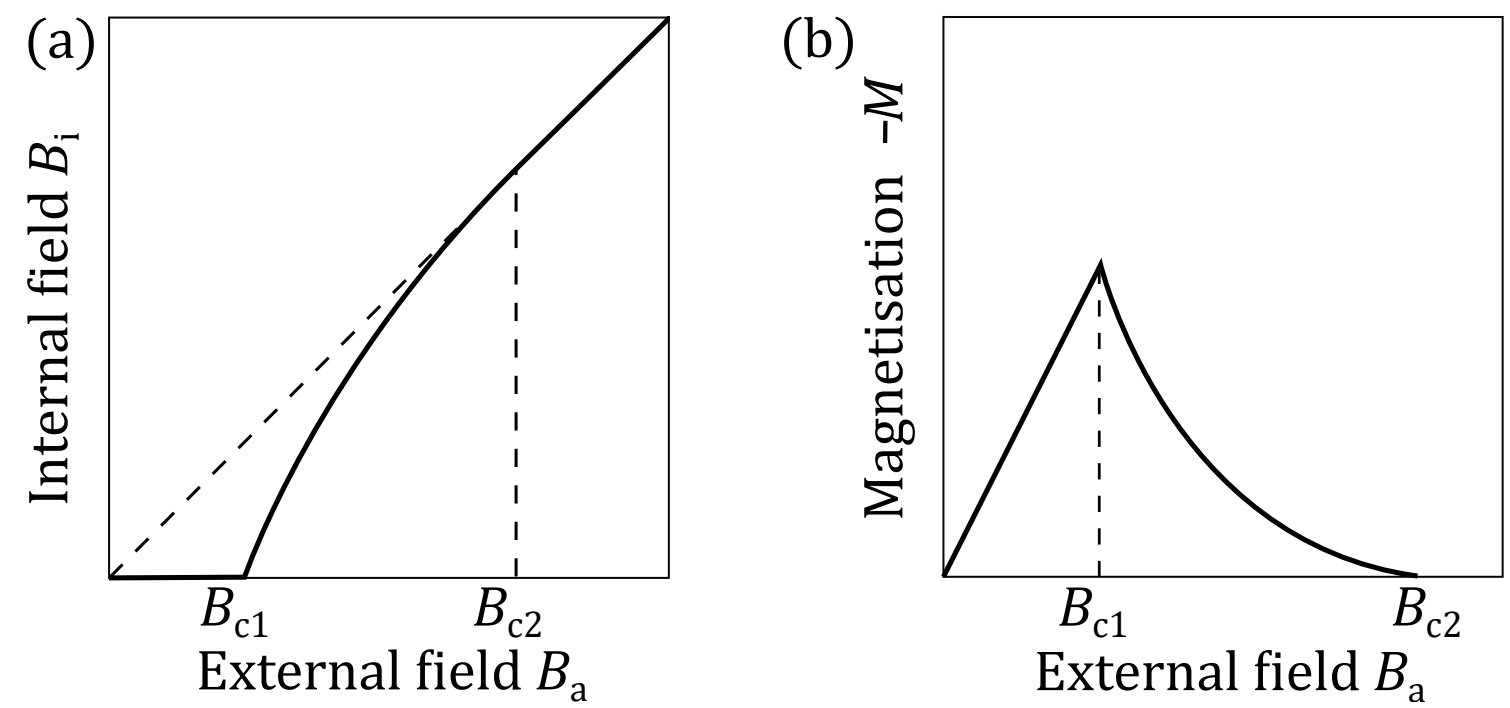

Figure 2.4: (a) Internal field, $B_{\mathrm{i}}$, and (b) negative magnetisation, $-M$, versus applied external field $B_{\mathrm{a}}$ for an ideal type II superconductor without demagnetisation. At first, the magnetic field is expelled until the lower critical field, $B_{\mathrm{c} 1}$, is reached. From there, magnetic flux lines penetrate the superconductor increasing the internal field and reducing the diamagnetic magnetisation until the upper critical field, $B_{\mathrm{c} 2}$, is reached and superconductivity is suppressed.

no superconductors with $\kappa<0.1$ [Dew01], which is one of the reasons for the late discovery of type II superconductors Abr73].

\section{Type II superconductors}

The behaviour of a superconductor significantly changes when the formation of non-superconducting areas is associated with a negative interface energy. As a result, it becomes energetically favourable to have the magnetic field penetrate the superconductor in form of magnetic flux lines, also known as vortices [Abr57, Abr73, Ens05, Tin96]. Figure 2.4(a) shows the internal field versus the externally applied field for a type II superconductor. At low applied fields, the magnetic field is still expelled as mandated by the Meissner-Ochsenfeld effect. However, after surpassing a certain threshold, the lower critical field $B_{\mathrm{c} 1}$, magnetic flux starts to enter the sample. This process continues until the whole sample is driven into the normal conducting state at the upper critical field, $B_{\mathrm{c} 2}$. This is further illustrated by the magnetisation versus applied field plot shown in figure 2.4(b). At first, the 

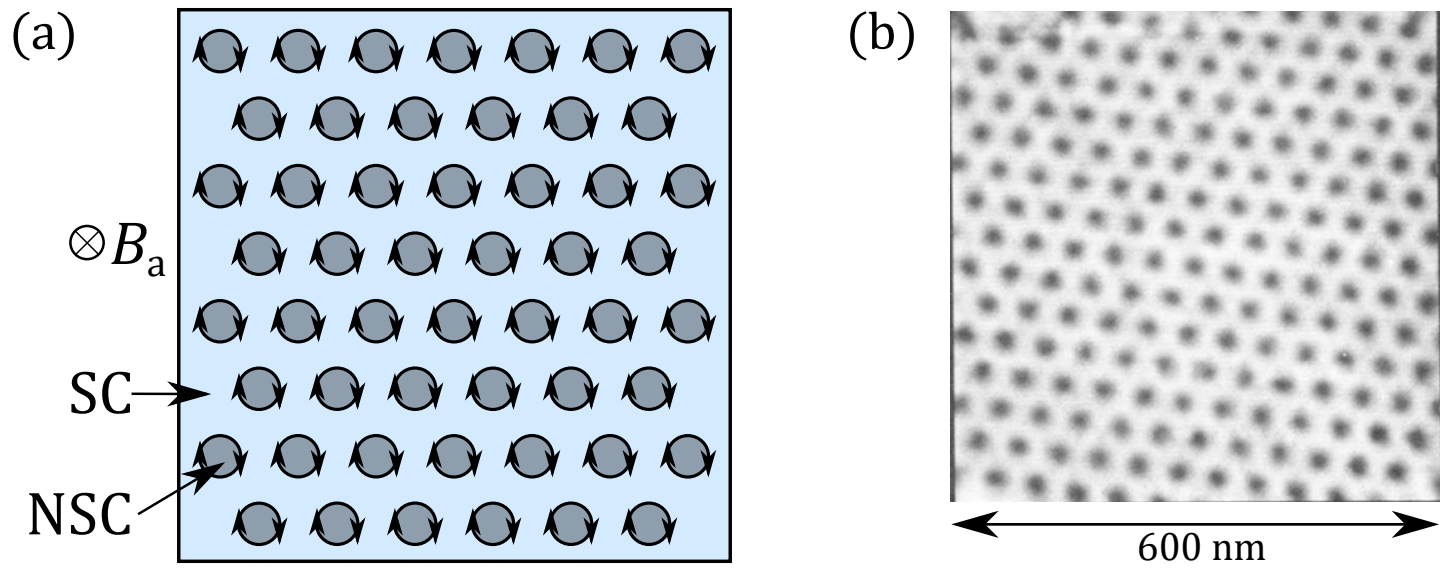

Figure 2.5: (a) Schematic of a type II superconductor penetrated by magnetic flux lines in the form of an Abrikosov lattice. The superconducting regions (SC) and nonsuperconducting (NSC) vortex cores are depicted in light blue and grey, respectively. (b) Experimental observation of the Abrikosov flux lattice by scanning tunneling microscopy (adapted from [Hes89]).

superconductor exhibits perfect diamagnetism until $B_{\mathrm{c} 1}$ is reached. After that, magnetic flux lines penetrate into the sample reducing the diamagnetic response until they are fully covering the superconductor at $B_{\mathrm{c} 2}$ and superconductivity is lost. All high-temperature superconductors and most compound superconductors are of type II. In terms of application, they are more promising due to their significantly larger critical field, which allows for the fabrication of coils that can produce magnetic fields of up to $45 \mathrm{~T}$ [Ugl19, Hah19].

This thesis is for the most part concerned with the properties of $\mathrm{FeSe}_{1-x} \mathrm{Te}_{x}$, which is classified as a type II superconductor. Because of this, the following sections are focused on the behaviour of type II superconductor.

\subsubsection{Abrikosov lattice}

Using GL theory, Abrikosov showed that due to the negative surface energy of type II superconductors, magnetic flux lines penetrate into the superconductor and form a specific energetically favoured lattice Abr57, Abr73]. This vortex lattice is often referred to as Abrikosov lattice or structure. Figure 2.5 shows a schematic diagram (a) and an actual scanning tunneling microscope image (b) [Hes89] of such an Abrikosov 


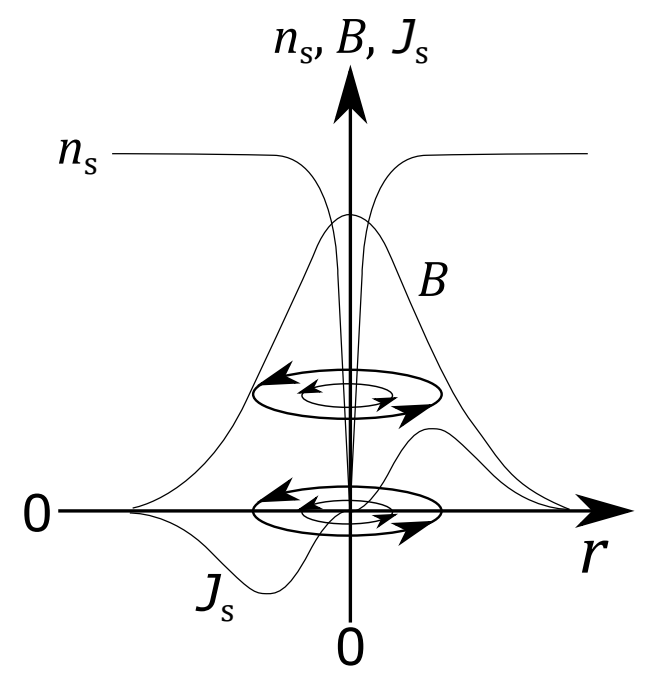

Figure 2.6: Evolution of the superconducting charger carrier density, $n_{\mathrm{s}}$, the magnetic field, $B$, and the superconducting current density, $J_{\mathrm{s}}$, in a magnetic flux line. This figure was adapted from Ens05.

lattice. It is worth noting, that this lattice is however rarely exhibited in many real type II samples as it requires a perfect, ideal, defect free sample [Ens05]. Typically, defects distort the lattice structure due to flux pinning, which will be introduced in the next section.

The structure and change in the physical properties of a cross-section of a magnetic flux line is depicted in figure 2.6. The core of a flux line is non-superconducting and has a width on the order of the coherence length. Around the core, a superconducting current circulates to conserve the magnetic flux - hence their name, magnetic vortices. One cannot miss the similarity of these vortices to the vortices of superliquid helium [Ens05]. In fact, it was the discovery of vortices in helium that led Abrikosov to publish his - at the time thought to be controversial - work Abr57, Abr73] .

\subsubsection{Vortex dynamics and critical current density in type II supercon- ductors}

The energy gap of the superconducting state and the formation of cooper pairs described by Bardeen-Cooper-Schrieffer (BCS) theory [Coo56, Bar57] dictate an upper limit for the superconducting current [Tin96, Dew01]. Specifically, once the kinetic 
energy of the cooper pair exceeds the cooper pair binding energy, the electrons 'depair' and superconductivity is lost [Tin96, Dew01]. This maximum current is therefore often denoted as the depairing current, $J_{\mathrm{d}}$, and is proportional to the energy gap, $\Delta$, and the Fermi velocity $v_{\mathrm{F}}$ :

$$
J_{\mathrm{d}}=\frac{n q \Delta}{m v_{\mathrm{F}}}
$$

Typical values of the depairing current are on the order of $10^{8} \mathrm{~A} / \mathrm{cm}^{2}$ [Dew01]. However, this limit is rarely reached in real type II superconductors [Dew01]. As introduced in section 2.1.5, magnetic flux lines penetrate into the superconductor once the lower critical field is exceeded. When a transport current is applied, these flux lines are affected by the Lorentz force density

$$
\boldsymbol{f}_{\mathrm{L}}=\rho \boldsymbol{E}+\boldsymbol{J} \times \boldsymbol{B}
$$

This force density accelerates the flux lines perpendicular to $\boldsymbol{J}$ and $\boldsymbol{B}$ with the resulting movement of the flux lines inducing an electric field parallel to the transport current density.

Based on this, type II superconductors should not be able to carry resistanceless currents unless a force acting against the Lorentz force were to prevent the acceleration of the vortices. Luckily, inhomogeneities in parameters such as $\lambda_{\mathrm{L}}, \xi, T_{\mathrm{c}}$, or $B_{\mathrm{c}}$ lead to so-called flux pinning [Ens05, Tin96, Dew01]. The resulting pinning force is what prevents the flux flow in type II superconductors and enables high, resistanceless transport currents for applications such as high-field magnets. In fact, almost all real samples exhibit flux pinning and it is very challenging to synthesise a sample without any flux pinning. This is due to the fact that any defect such as impurities, grain boundaries, secondary phases, or basically any structural defect can act as a pinning centre.

Figure 2.7 qualitatively illustrates flux pinning for the simple case of a single vortex (red bar) and a single non-superconducting defect site (grey circle). As is the case 

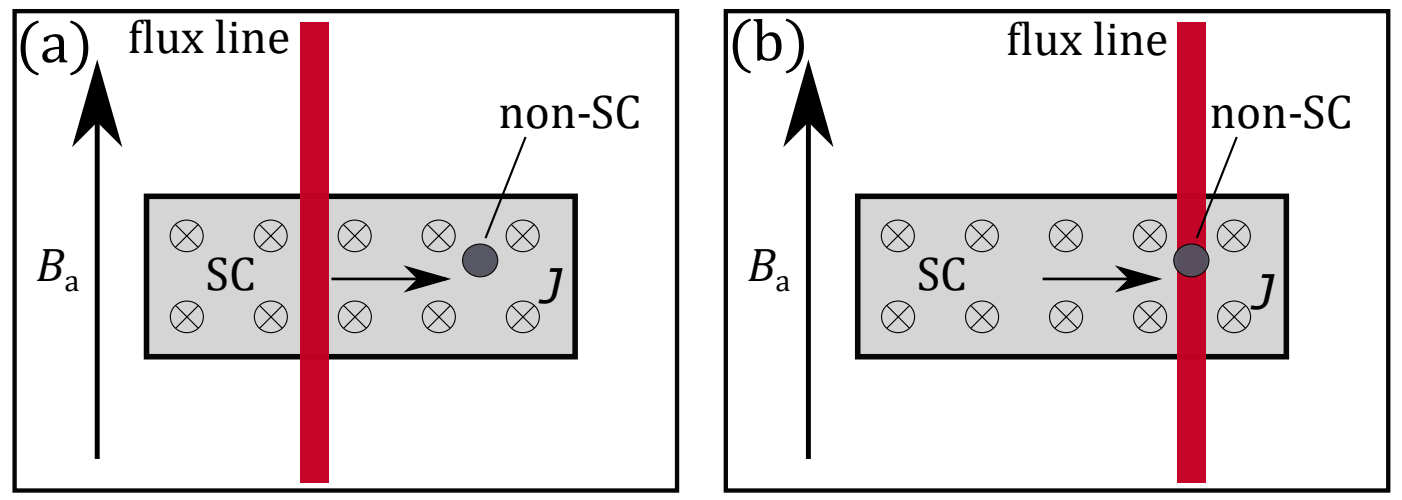

Figure 2.7: Schematic to illustrate flux pinning of a single magnetic vortex (red bar) on a non-superconducting (non-SC) defect. At first (a), the vortex is accelerated perpendicularly to $B$ and $J$ and moves towards the non-superconducting defect. In (b), the normal conducting core covers the non-superconducing defect and further movement is prevented by the energy barrier that the vortex would have to overcome.

for everything in nature, every system always strives for the lowest energy state. In a type I superconductor, this lowering of energy is obtained by transitioning from the non-superconducting state to the superconducting state and expelling all magnetic field up to the critical field $B_{\mathrm{c}}$. In a type II superconductor, the scenario is similar, but due to the small coherence length in comparison to the penetration depth, it becomes favourable to let some magnetic flux penetrate in form of flux lines. The area of the normal conducting core of each flux line therefore represents an energy penalty equal to the volume times the condensation energy density. If there is an area inside the sample that is already non-superconducting to begin with, it becomes energetically favourable to centre a vortex on this defect. This is illustrated in figure 2.7. At first (a), the vortex is being pushed perpendicularly to the transport current density, $\boldsymbol{J}$, by the Lorentz force until it reaches the non-superconducting defect site (b). Any further motion away from this defect site now comes with an energy penalty: the vortex is pinned.

The above gave a brief introduction to flux pinning for the simple case of a single flux line and a single non-superconducting defect. In general, flux pinning is a very complicated topic that is still heavily researched especially because of its tremendous importance for the application of superconductors. For example, the shape and 
dimensionality of the defects play a crucial role as it can lead to strong anisotropic behaviour depending on the orientation of the transport current in relation to the defect. Another important factor is the distribution of the defects sites and the elasticity of the vortices. Lastly, depending on the penetration depth and coherence length of the superconductor, vortex-vortex interaction becomes increasingly important at higher fields. For a more detailed description of flux pinning, the reader is directed to the work of Senoussi [Sen92] and Blatter et al. [Bla94], which discuss flux pinning in great depth.

\subsubsection{The critical state model (Bean Model)}

Based on equation 2.13, the Lorentz force density is proportional to the transport current density, $\boldsymbol{J}$. Therefore, with increasing transport current, the Lorentz force will eventually overcome the pinning force. This is what determines the critical current density, $J_{\mathrm{c}}$, in type II superconductors and is typically one or two orders of magnitudes below the aforementioned depairing current, $J_{\mathrm{d}}$ [Dew01, Tin96]. The point at which the Lorentz force density equals the pinning force density

$$
f_{\mathrm{L}}=\boldsymbol{f}_{\mathrm{p}}
$$

is often referred to as the critical state first described by Bean in 1962 [Bea62, Bea64. Instead of describing the complicated microscopic pinning behaviour of a superconductor, Bean's model directly relates the macroscopic magnetisation of the superconductor to $J_{\mathrm{c}}$ [Bea62, Bea64]. The main assumptions of Bean's model are that there is a limiting critical current that a superconductor can carry and that any electromotive force will induce this full current [Bea62, Bea64]. Furthermore, the original Bean model assumes that the critical current density is independent of the magnetic field, $J_{\mathrm{c}}(B)=J_{\mathrm{c}}=$ constant, leading to a constant vortex gradient. Based on this, the magnetic field (a) and current density (b) profile of a superconducting slab of thickness $d$ in an increasing parallel applied field can be described by the 
(a)

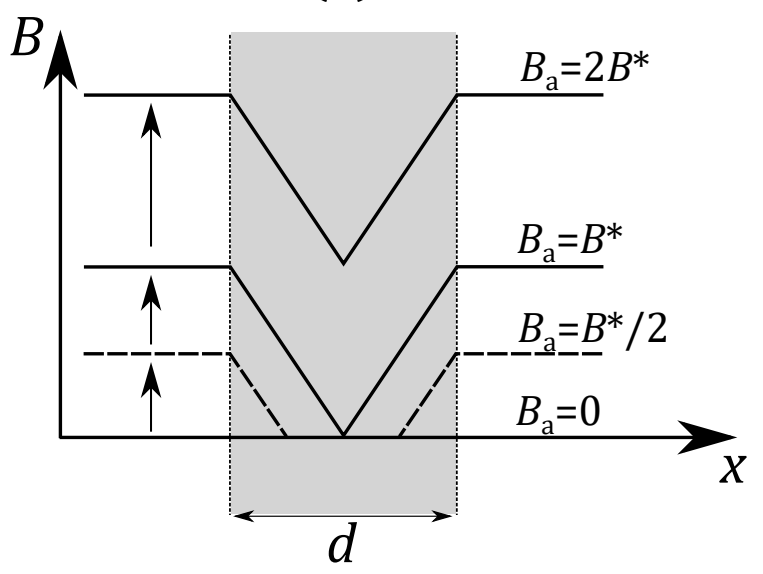

(b)

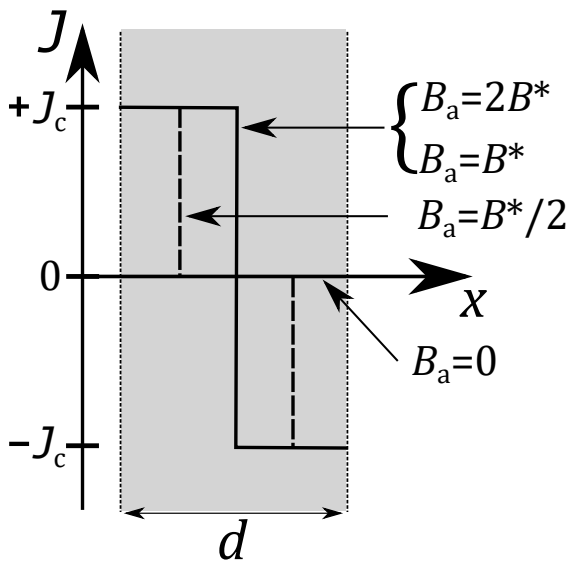

Figure 2.8: (a) Magnetic field profile, $B(x)$, according to Bean's model for the case of a slab of thickness $d$ in increasing field, $B_{\mathrm{a}}$. (b) Current density in the slab as a result of the magnetisation gradient at different applied fields.

Bean model as depicted in figure 2.8.

Starting at low fields above $B_{\mathrm{c} 1}\left(B_{\mathrm{a}}=B^{*} / 2\right.$, dashed line $)$, flux starts to penetrate into the sample with the centre of the sample remaining flux free. The established flux gradient leads to supercurrents flowing in the part of the sample that is penetrated by the flux with the centre not carrying any current. As the external field is increased, the flux continues to penetrate deeper into the sample until it reaches the centre and the whole sample is magnetised $\left(B_{\mathrm{a}}=B^{*}\right)$. Now, the current runs in the whole sample with a magnitude proportional to the flux gradient. Further increases in the applied magnetic field $\left(B_{\mathrm{a}}>B^{*}\right)$ do not change the flux gradient and, equally, the current density.

When the external field is now decreased, flux starts to exit the sample from the edges and the sign of the flux gradient changes (figure 2.9(a)), $B_{\mathrm{a}}>0$ ). Consequently, the current in the region close to the edge now runs in the opposite direction following the sign change of the flux gradient. This is depicted in figure 2.9(b) for $B_{\mathrm{a}}=B^{*}$. At this field, the current density in the inner part of the sample is still running in the same direction as the flux gradient in that region remains unchanged. Interestingly, when the external field is further decreased to $B_{\mathrm{a}}=0$, flux is still trapped in the 
(a)

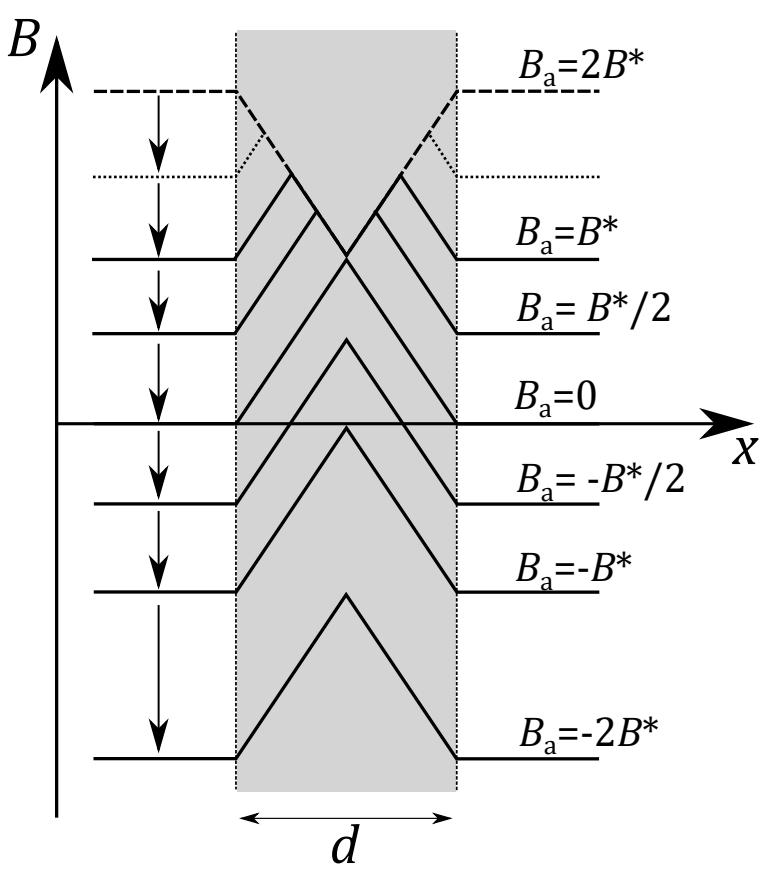

(b)

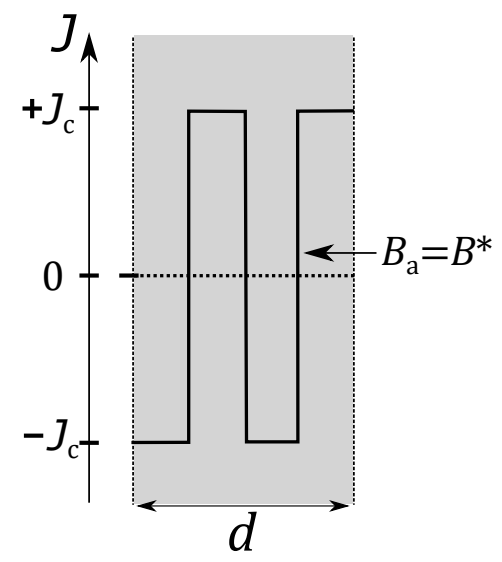

(c)

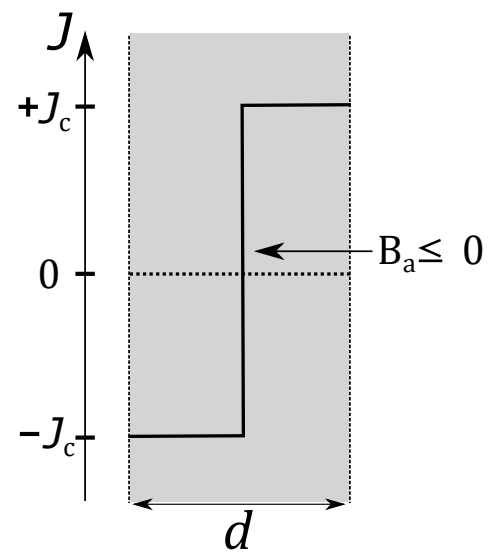

Figure 2.9: (a) Magnetic field profile, $B(x)$, according to Bean's model for the case of a slab of thickness $d$ in decreasing field, $B_{\mathrm{a}}$. (b) Current density in the slab as a result of the magnetisation gradient at $B_{\mathrm{a}}=B^{*}$. (c) Current density in the slab as a result of the magnetisation gradient at $B_{\mathrm{a}} \leq B^{*}$.

superconductor and hence currents remain running in the superconductor (figure 2.9 (c)). This phenomenon of trapped flux is, for example, used in bulk magnets that can trap magnetic fields of up to $17.6 \mathrm{~T}$ [Dur18].

When the field is increased but with opposite sign, flux again enters from the edges of the sample. This flux is of opposite polarisation as the trapped flux in the centre and therefore leads to flux annihilation at the interface. Eventually, at $B_{\mathrm{a}}=-B^{*}$, the trapped flux is fully removed and the whole sample is penetrated by flux of the same polarity. It is worth noting though, that the current density does not change during this process as the flux gradient remains unchanged.

Figure 2.10 depicts the resulting magnetic hysteresis loop (MHL) based on the Bean 


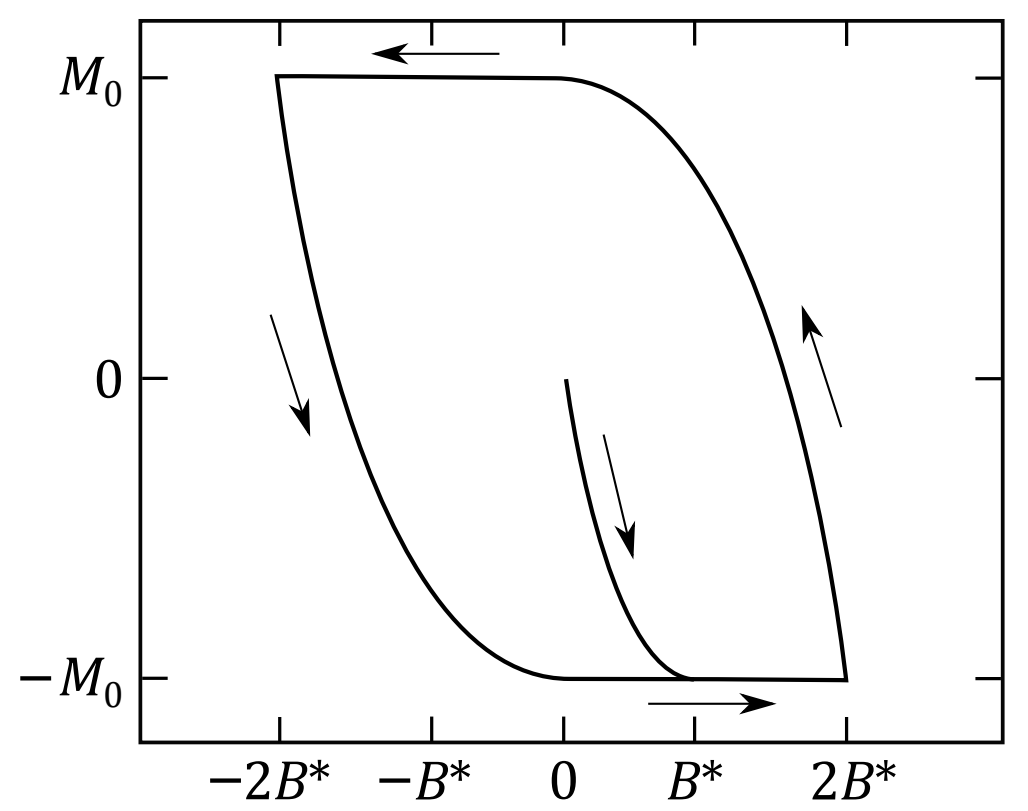

Figure 2.10: Theoretical magnetic hysteresis loop based on the Bean Model (adapted from Shantsev et al. [Sha99]).

model for the characteristic fields introduced in figure 2.8 and 2.9 . Once a field $B_{\mathrm{a}}>B^{*}$ is applied, the magnetisation reaches its maximum absolute value, $\left|-M_{0}\right|$. Further increases in the applied magnetic field do not change the magnetisation. When the field is now reduced, flux starts to exit the sample from the edges, a flux gradient of opposite sign forms, and the magnitude of the magnetisation decreases. Eventually, once the opposite-sign flux gradient has penetrated more than $50 \%$ of the superconductor, the magnetisation again increases reaching a fully reversed flux gradient at $B_{\mathrm{a}}=0$. The sample is now magnetised with opposite polarity $M=M_{0}$. It is worth noting that the magnetisation $M_{0}$ is the average magnetisation of the whole superconductor. Given that the origin of the magnetisation of a superconductor is the aforementioned flux gradient within the sample, one must always be aware of the difference between the local magnetisation that is a function of the position and the orientation of the applied field and the average magnetisation of the whole sample that is measured in typical SQUID or VSM measurements.

The initial model proposed by Bean undeniably oversimplifies the hysteresis cycle of type II superconductors. For instance, the assumption of a magnetic-field- 


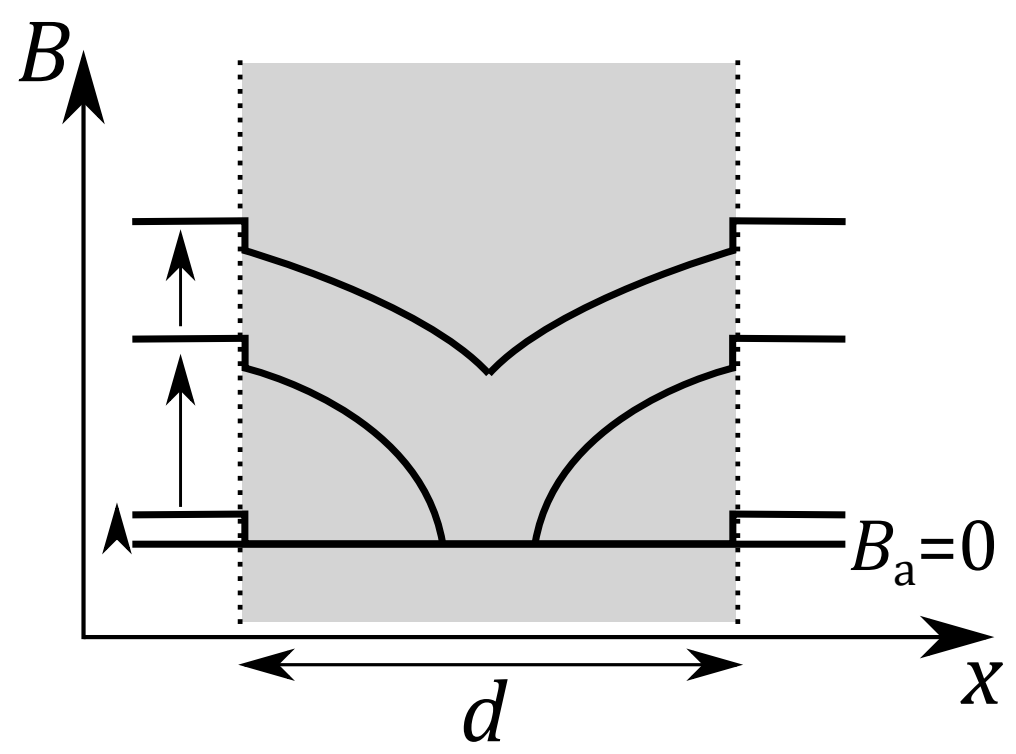

Figure 2.11: Magnetic field profile, $B(x)$ of a type II superconductor of width $d$ when taking into account surface effects and a magnetic-field-dependent $J_{\mathrm{c}}(B)$.

independent critical current density is essentially never correct for real superconductor. This field-dependency of $J_{c}$ was taken into account by Kim et al. Kim63 and Fietz et al. [Fie64 who described $J_{c}$ as functions of $B$ and two positive parameters $J_{\mathrm{c} 0}$ and $B_{0}$ :

$$
\begin{aligned}
& J_{\mathrm{c}}=\frac{J_{c 0}}{1+B / B_{0}} \quad \text { (Kim Model) } \\
& J_{\mathrm{c}}=J_{c 0} \cdot \exp \left(-B / B_{0}\right) \quad \text { (Fietz Model) }
\end{aligned}
$$

Furthermore, Bean's model neglects surface effects that, for instance, lead to a barrier for flux entry Cam01]. The resulting magnetisation profile for the more realistic behaviour is depicted in figure 2.11.

Nevertheless, the Bean model is still of extreme importance as it allows for the estimation of the critical current density based on magnetic measurement. Specifically, one can estimate the critical current density based on the width of the hysteresis loop and the geometry of the sample. In the case of a thin slab with the smallest 
dimension parallel to the applied field, $J_{\mathrm{c}}$ can be estimated as

$$
J_{\mathrm{c}}(B)=\frac{20 \Delta M(B)}{a(1-a / 3 b)}
$$

where $a$ is the width of the sample, $b$ the length of the sample with $b>a$, and $\Delta M(B)$ is the difference in magnetisation between the magnetisation in increasing- $\left(M_{\text {inc }}(B)\right)$ and decreasing applied field $\left(M_{\mathrm{dec}}(B)\right)$ at a given applied field of the hysteresis loop $\Delta M(B)=\left|M_{\text {inc }}(B)-M_{\text {dec }}(B)\right|[$ Bea62, Bea64, Joh95].

\subsubsection{Anderson flux creep}

In 1962, Anderson And62, And64 expanded on the idea of the critical state and showed that at $T>0 \mathrm{~K}$, the critical state is not a static state but of dynamic nature due to thermally activated flux creep. Specifically, magnetic flux lines or typically flux bundles jump over the activation barrier, $U$, with a jump rate of

$$
\nu=\nu_{0} \cdot \exp \left(-U / k_{\mathrm{B}} T\right)
$$

where $v_{0}$ is the attempt frequency [Bea69]. In the simple Anderson-Kim model, the activation barrier, $U$, is described with a linear $J$-dependence

$$
U=U_{0}\left(1-\frac{J}{J_{0}}\right)
$$

where $U_{0}$ is the activation barrier in absence of a driving force and $J_{0}$ the current den-

sity at which the barrier vanishes [Bea69, Yes96]. From this follows the logarithmic decay of the current density with time

$$
J=J_{\mathrm{c} 0}\left(1-\frac{k_{\mathrm{B}} T}{U_{0}} \ln \left(t / t_{0}\right)\right)
$$

where $t_{0}$ is the effective attempt time [Yes96, Ges89, Fei89]. Figure 2.12 shows the original data of Kim et al. who measured the decay of the persistent currents in a 


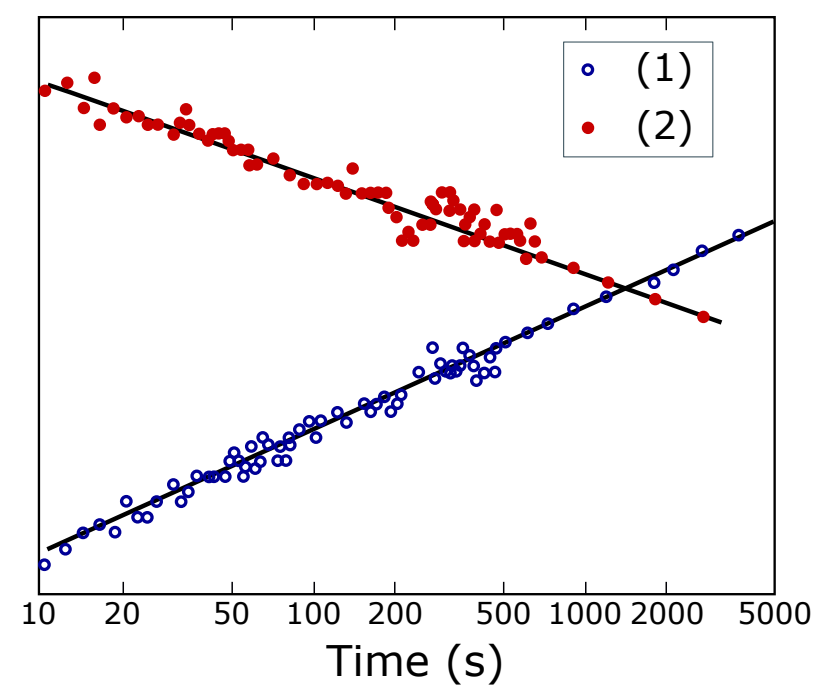

Figure 2.12: Decay rate of persistent currents induced in a Nb-Zr tube. Data (1) was taken at the shielding portion of the magnetisation curve and (2) on the trapping portion. This figure is adapted from Kim et al. Kim62].

Nb-Zr tube with time [Kim62]. As vividly shown in the graph, both the ascending(1) and descending (2) applied field branch exhibit a logarithmic decay of the current density with time as described by equation 2.20

In general, the measurement of the relaxation of $J(t)$ or equally $M(t)$ is of special interest since it contains information on the activation barrier of a compound through equation 2.20 .

$$
S \equiv \frac{1}{M} \frac{\mathrm{d} M}{\mathrm{~d} \ln (t)}=\frac{\mathrm{d} \ln (M)}{\mathrm{d} \ln (t)}=\frac{\mathrm{d} \ln (J)}{\mathrm{d} \ln (t)}=\frac{-k_{\mathrm{B}} T}{U_{0}-k_{\mathrm{B}} T \ln \left(t / t_{0}\right)}
$$

By measuring the decay of the magnetisation with time and fitting the slope, the effective barrier, $U_{\text {eff }}=U_{0}-k_{\mathrm{B}} T \ln \left(t / t_{0}\right)$ can directly be extracted from the data. In the case $U_{0} \gg k_{\mathrm{B}} T \ln \left(t / t_{0}\right)$, the slope can be used to estimate the activation barrier that relates to the pinning properties of the sample.

\section{Surface and bulk relaxation}

There are two types of pinning that are most prominent in the samples investigated in this thesis: surface- and bulk pinning. As a result, there are also two characteristic 

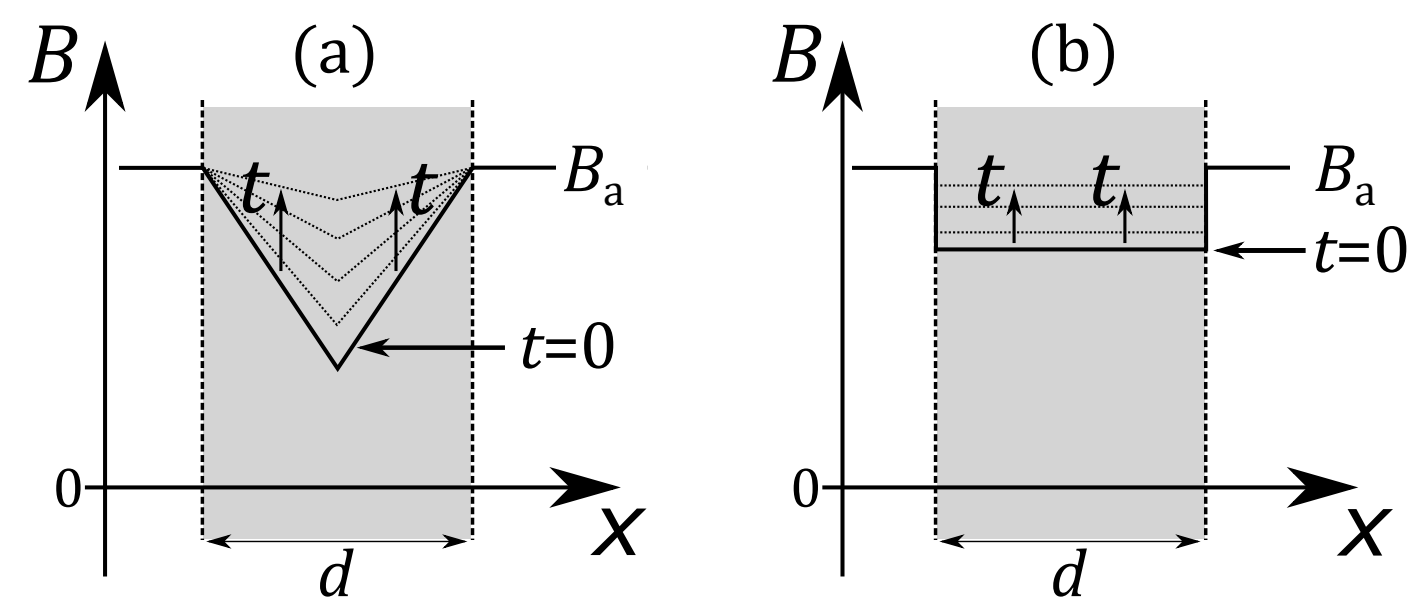

Figure 2.13: Magnetic relaxation profile for Bean-like, bulk pinning (a) and surface pinning (b) Bur93].

relaxation processes for the two different pinning mechanisms. This is best illustrated by the schematic of solely bulk- (a) or surface relaxation (b) shown in figure 2.13. For simplicity and ease of understanding, the bulk magnetisation gradient is assumed to be constant as described by the original Bean model [Bea62, Bea64].

In the case of bulk relaxation (a), the initial magnetisation gradient $(t=0)$ is largest and continuously decreases with time due to flux creep. The physical origins of bulk pinning are typically defects in the bulk of the sample. For instance, earlier mentioned non-superconducting regions can act as pinning centres if their size is comparable to the coherence length of the superconductor and thus the vortex core size. For surface pinning, one possible origin is the so-called Bean-Livingston surface barrier [Bea64a]. This barrier arises from the competing attracting force due to mirror vortices as dictated by the surface boundary condition and the repelling force due to screening currents [Bea64a, Yes96]. This type of barrier has been shown to be more prominent in high-temperature superconductors [Kon92, Yes96] and is thus also of relevance to this thesis. The magnetisation profile due to a surface barrier exhibits a very sharp drop at the surface boundary of the superconductor. With time, the magnetisation is reduced due to the flux creeping over the surface barrier as depicted in figure 2.13(b).

In general, it is important to note that a reduction of the magnetisation with time 
due to flux creep is only present in the case of type II superconductors, which exhibit an irreversible magnetisation, $M_{\text {irr }}$, as a results of flux pinning. For instance, screening currents that oppose the change in field in the Meissner-Ochsenfeld state do not exhibit magnetic relaxation and the resulting magnetisation is considered part of the reversible equilibrium magnetisation, $M_{\mathrm{eq}}$ [Sen91, Che93, Yes96]. Consequently, relaxation measurement can also help to identify the origin of the observed magnetisation. In the case of negligible surface pinning, the two contributions can be isolated from MHL measurements as

$$
\begin{aligned}
& M_{\mathrm{eq}}=\frac{M_{\mathrm{dec}}+M_{\mathrm{inc}}}{2} \\
& M_{\mathrm{irr}}=\frac{M_{\mathrm{dec}}-M_{\mathrm{inc}}}{2}
\end{aligned}
$$

[Yes96]. Because flux creep was first investigated in the context of low-temperature superconductors and before the discovery of high-temperature superconductors [And62, And64, Kim62, the effect was initially found to be minute and the currents could be thought of as being 'quasi' persistent [Kim62, Tin96, Yes96]. However, for high-temperature superconductors, flux creep is increasingly important because of the small coherence length and the higher operating temperatures [Dew88, Yes88].

\subsubsection{Sweep-rate-dependence of MHLs}

One of the main consequences of flux creep is that the magnetisation measured in a MHL measurement is not only dependent on the history of the sample and the applied magnetic field, but also on the effective time of the measurement, $M(B, t)$. Specifically, the time between the last change in field and the rate at which the applied field is changed, $K=\partial B / \partial t$, relates to the extend of the relaxation. Therefore, the critical current density determined by MHL measurements using the Bean model is always a function of time as well. In practice, this means that it is vital to consider the measurement time when comparing $J_{\mathrm{c}}$ among different reports. This has been demonstrated in the literature [Pus90, Sun92] and is nicely illustrated by the 
experimental work of Senoussi et al. for the case of a YBCO sample [Sen92]. The faster the applied field was swept, the larger the measured magnetisation and thus the estimated $J_{c}$. Consequently, the lowest magnetisation is measured in persistentfield measurements in which the field is first stabilised before every measurement. Because of this, one must always take great care when comparing the absolute values of $J_{\mathrm{c}}$ obtained from MHL measurements in the literature.

\subsection{Iron-based superconductors}

Since the discovery of superconductivity in the cuprates [Bed86], high-temperature superconductivity was thought of as a synonym for cuprates. This changed after the observation of superconductivity in the iron-based compound LaOFeP by the Hosono group in 2006 [Kam06]. The critical temperature of the first member of the family of iron-based superconductor was still low with $T_{\mathrm{c}}=4 \mathrm{~K}$. However, this was quickly improved upon with the discovery of superconductivity in $\mathrm{LaFeAsO}_{1-x} \mathrm{~F}_{x}$, which exhibited a $T_{\mathrm{c}}$ of $26 \mathrm{~K}$ [Kam08] that could even be increased to $43 \mathrm{~K}$ by applying $4 \mathrm{GPa}$ of pressure [Joh15]. At this point, $43 \mathrm{~K}$ was the highest reported $T_{\mathrm{c}}$ outside the copper-oxide based superconductors. This observation quickly sparked interest in iron-based compounds and was rapidly followed by the discovery of several superconducting iron-based compounds such as $\mathrm{Ba}_{1-x} \mathrm{~K}_{x} \mathrm{Fe}_{2} \mathrm{As}_{2}$ [Rot08, LiFeAs Wan08, and FeSe [Hsu08]. Figure 2.14 shows a brief historical timeline of the rapid discoveries of additional members of the iron-based superconductor family following the initial discovery in 2006.

\section{$2.3 \quad \mathrm{FeSe}_{1-x} \mathrm{Te}_{x}$}

This thesis is focused on the structural and superconducting properties of $\mathrm{FeSe}_{1-x} \mathrm{Te}_{x}$, which is commonly referred to as the 11-type of the iron-based superconductor family [Joh15]. Therefore, a more detailed introduction to the properties 


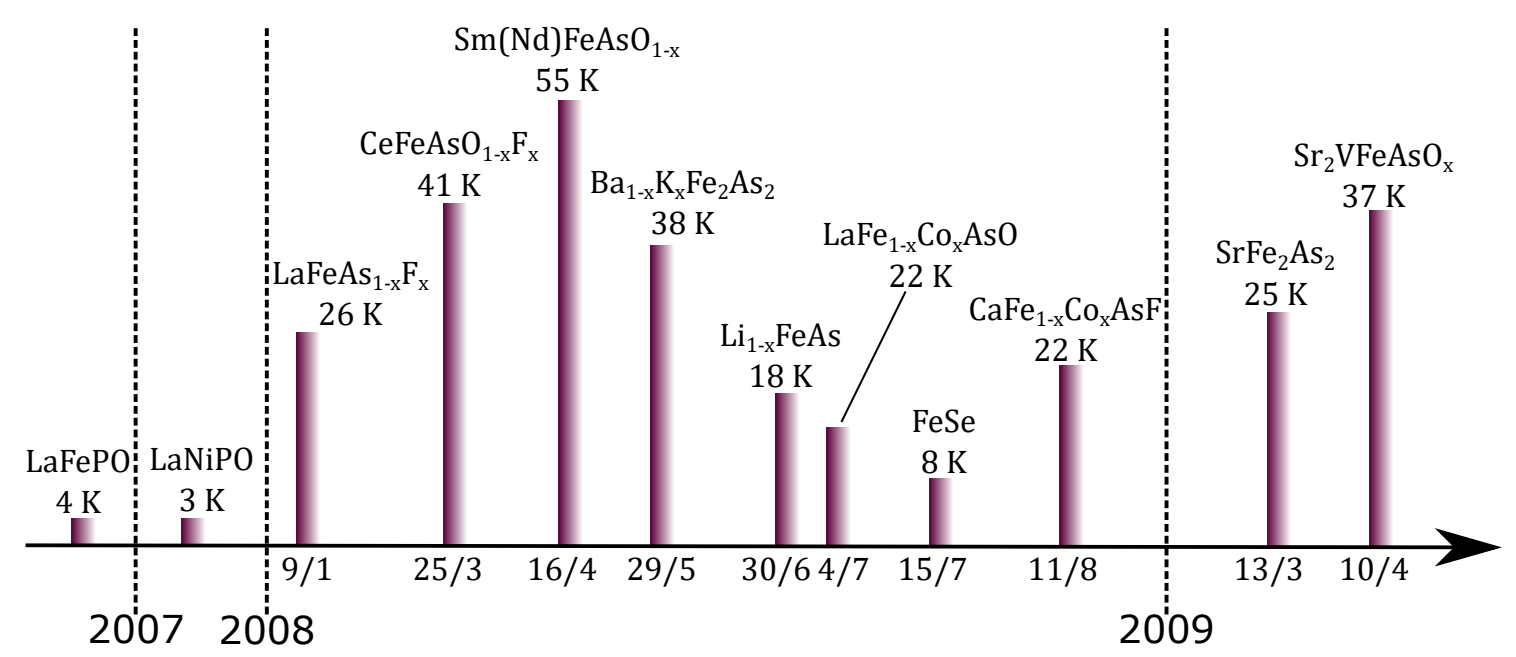

Figure 2.14: Brief historical timeline of the rapid discoveries of several iron-based superconductors following the initial discovery of LaFePO in 2006. This figure is adapted from [Joh15.

of $\mathrm{FeSe}_{1-x} \mathrm{Te}_{x}$ will be given below. First, the crystal structure and the inevitable presence of interstitial excess iron in this compound is discussed. Following this, a Te-doping-dependent superconducting phase diagram based on four reports from the literature is presented. Lastly, an introduction to annealing, the main focus of this thesis, and the current state of the literature is given.

\subsubsection{Crystal structure and excess iron}

Figure 2.15(a) shows the crystal structure of $\mathrm{FeSe}_{1-x} \mathrm{Te}_{x}$. The structure is characterised by a layered, anti-PbO-type structure (Space group No.129, P4/nmm) build of $\mathrm{Fe}(\mathrm{Fe}(1)$, yellow spheres) and Se or Te atoms (grey spheres). Additionally, the synthesis of this compound also invariably leads to the presence of strongly magnetic, interstitial excess iron [Fru75, Bao09, Liu09, Zha09] depicted by the purple spheres $(\mathrm{Fe}(2))$ in the figure. This results from the need for excess iron for the formation of the anti-PbO-type phase in the parent compound (see $\beta$-phase in figure 2.15(b) Oka91]) and was recently verified for the superconducting phase by Bao et al. using neutron powder diffraction [Bao09]. In general, obtaining the right phase of $\mathrm{FeSe}_{1-x} \mathrm{Te}_{x}$ is quite challenging and requires a properly tuned synthesis temperature- and time- 

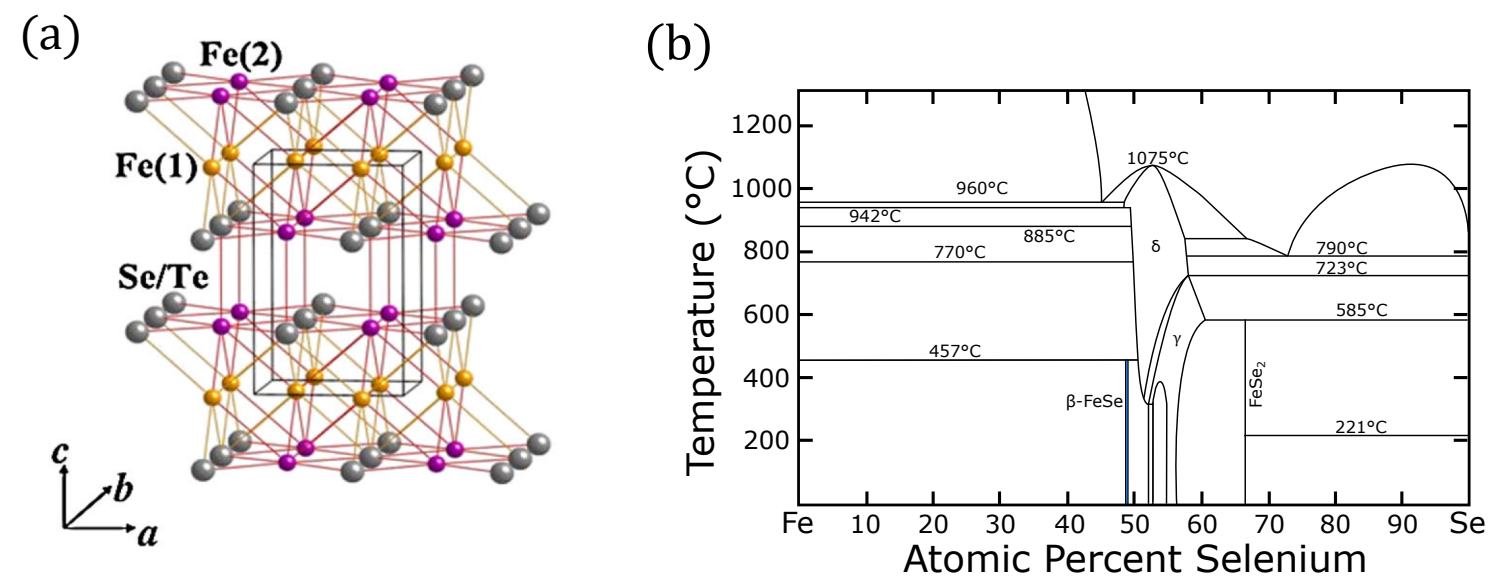

Figure 2.15: (a) Drawing of the layered crystal structure of $\mathrm{FeSe}_{1-x} \mathrm{Te}_{x}$. The grey spheres depict the Se and Te atoms, the yellow spheres, Fe(1) atoms, and the purple spheres interstitial excess iron, $\mathrm{Fe}(2)$ (this figure is adapted from [Bao09]). (b) Phase Diagram of FeSe (adapted from [Oka91]). The superconducting anti-PbO phase ( $\beta$ phase, light blue) only forms with excess iron in a small temperature window below $457^{\circ} \mathrm{C}$.

profile. This is best illustrated in the phase diagram of the parent compound shown in 2.15(b), where the small synthesis temperature and Se concentration window of the superconducting $\beta$-phase is marked in light blue. Besides the right temperature, the formation of the superconducting phase also requires a small amount of excess iron. As will be shown in this thesis, the presence of excess iron plays a critical part in the magnetic and superconducting properties of this compound and differentiates this family of iron-based superconductors from the other families.

\subsubsection{Superconducting phase diagram}

The superconducting phase diagram of tellurium doped $\mathrm{FeSe}_{1-x} \mathrm{Te}_{x}$ has been investigated independently by several authors [Hu12a, Sun16, Kaw12, Noj10 as summarised in figure 2.16. Note that for ease of understanding and because of the doping-dependence of this compound, the phase diagram is not plotted as function of the tellurium doping $(x)$ but the selenium concentration $(1-x)$.

Overall, the phase diagram shows a large variance in the reported $T_{\mathrm{c}}$ for a given selenium concentration. In the case of Noji et al., even the $T_{\mathrm{c}}$ of several samples from 


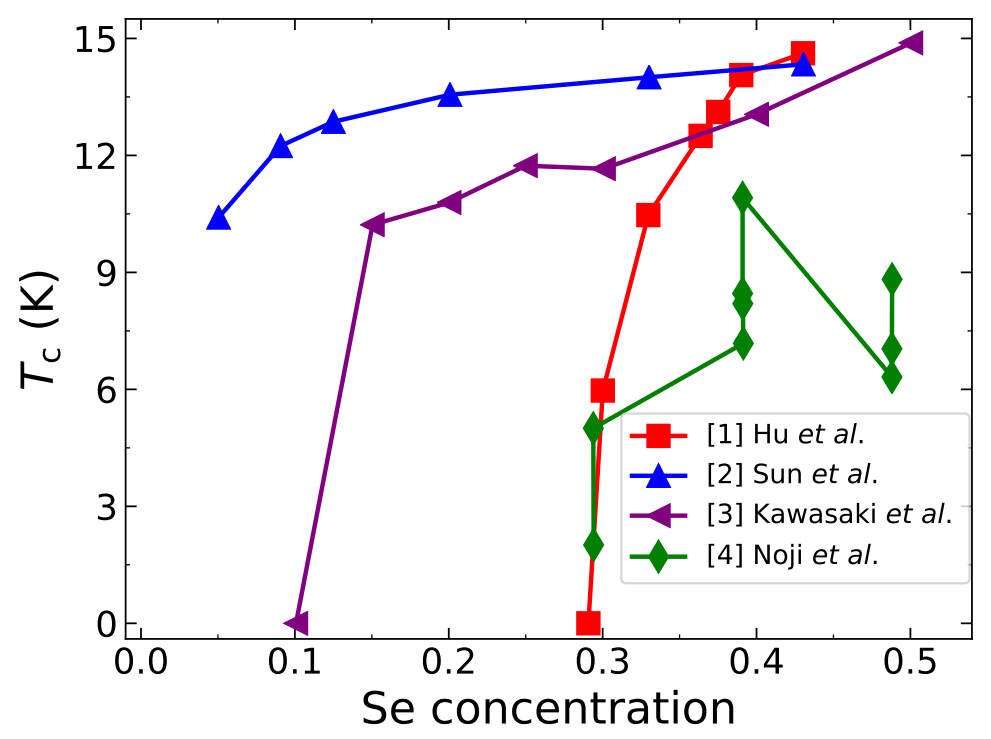

Figure 2.16: Plot of $T_{\mathrm{c}}$ versus the Se concentration for as-grown $\mathrm{FeSe}_{1-x} \mathrm{Te}_{x}$ single crystals from the literature [Hu12a, Sun16, Kaw12, Noj10.

the same report varies between $7.2 \mathrm{~K}$ and $10.9 \mathrm{~K}$. Nevertheless, there is a general upward trend of $T_{\mathrm{c}}$ with increasing selenium concentration from non-superconducting FeTe to $\mathrm{FeSe}_{0.5} \mathrm{Te}_{0.5}$ with a maximum $T_{\mathrm{c}}$ of up to $15 \mathrm{~K}$ as reported by Kawasaki et al. Kaw12. Higher selenium concentrations are typically avoided since the superconducting properties start to worsen when increasing the selenium concentration past $\mathrm{FeSe}_{0.5} \mathrm{Te}_{0.5}$. Additionally, there is a miscible gap for selenium concentrations between 0.7 and 0.95 that prevents the formation of a homogenous crystal structure because of phase separation [Miz10]. The initially discovered undoped, parent compound, FeSe, has a $T_{\mathrm{c}}$ of $8 \mathrm{~K}$ [Hsu08] (not shown in the figure).

The large variance in $T_{c}$ observed in this family of superconductors is likely due to the aforementioned interstitial excess iron, which has been shown to suppress superconductivity in this compound by several authors McQ09, Zha09, Lei10, Liu09, Don11]. More specifically, different synthesis conditions have most likely resulted in differences in the interstitial excess iron concentration and thus differences in the superconducting properties between the different reports. 


\subsubsection{Annealing}

Annealing typically refers to a process in which the sample is heated up to a temperature below the melting point, but high enough to promote the movement of out-ofplace atoms to a lattice spot where they belong and thus improving the homogeneity and crystallinity of the sample [Lau14]. Most often, annealing is performed in vacuum or an inert atmosphere to prevent any reaction of the sample with the annealing atmosphere. For example, the presence of oxygen in the annealing atmosphere may cause the formation of oxides on the sample.

The effect of annealing on $\mathrm{FeSe}_{1-x} \mathrm{Te}_{x}$ single crystals has also been studied by several authors. Improvements in the superconducting properties have been reported for a variety of annealing atmospheres such as oxygen [Kaw12, Sun13d], iodine [Rod11, Sun13d], chalcogens [Kos13, Sun13a, Sun13b], pnictogens [Zho14, Hu12a, Sun13d, air [Don11], and under vacuum [Noj10, Kaw12, Sun13d]. The observed improvement of the superconducting properties has been attributed to several processes by different authors, for instance a more homogeneous distribution of Se and Te after vacuum annealing [Noj10, intercalated oxygen acting as a hole donor compensating the electrons of the interstitial excess iron [Kaw12, oxygen speeding up a phase transition from the tetragonal $\mathrm{PbO}$-type phase to the hexagonal NiAs-type phase [Hu12b], or most prominently a reduction in interstitial excess iron [Don11, Sun14, Tam16, Rod11, Zho14].

The removal of excess iron in $\mathrm{Fe}_{1+y} \mathrm{Se}_{0.4} \mathrm{Te}_{0.6}$ after annealing in oxygen was investigated in detail by Sun et al. in 2014 Sun14. By studying the cross section of as-grown and oxygen-annealed single crystals via scanning tunneling microscopy (STM), they were able to show that oxygen annealing largely reduces the amount of excess iron in the sample. Figure 2.17 shows their STM images of the as-grown (a) and oxygen-annealed sample (b,c). The as-grown sample exhibits several bright white spots indicative of excess iron. After oxygen annealing, the number and brightness of the white spots is greatly reduced indicating a reduced excess iron concentra- 

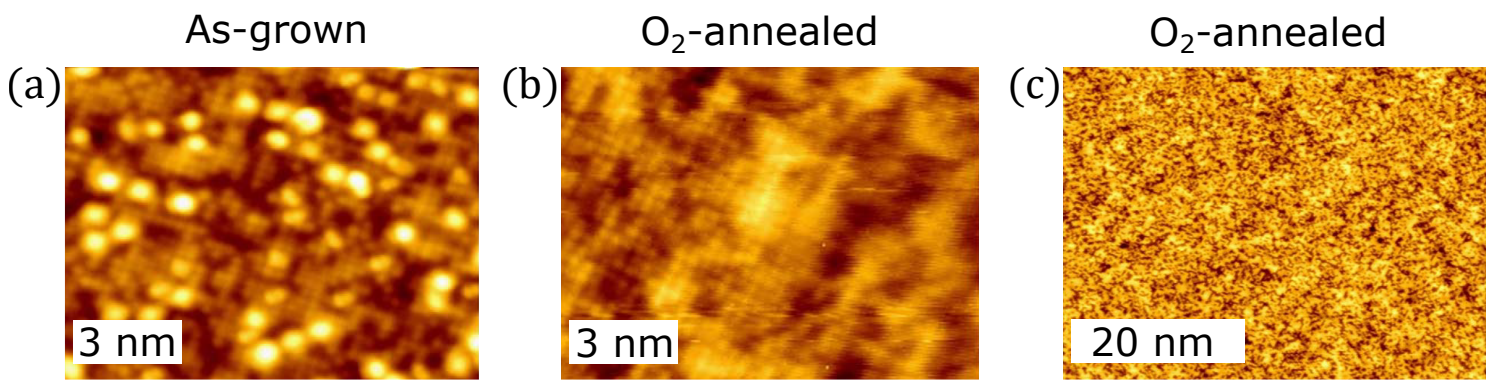

Figure 2.17: STM map of as-grown (a) and oxygen-annealed (b,c) $\mathrm{FeSe}_{0.4} \mathrm{Te}_{0.6}$ single crystals. This figure is adapted from [Sun14].

tion. This was not only the case for the region depicted in the figure but for several different regions of their sample [Sun14].

Overall, the effect of annealing on the superconducting properties of $\mathrm{FeSe}_{1-x} \mathrm{Te}_{x}$ has been reasonably well studied in the literature [Hu12a, Sun16, Kaw12, Noj10, Sun19]. However, there are contradicting reports on the effect of annealing in different atmospheres [Hu12a, Noj10, Sun13d]. Furthermore, only a few reports analyse the structural changes that lead to the observed improvement in the superconducting properties [Hu12b, Lin15, Yam16]. For instance, the formation of a blue surface layer on the surface of the single crystals after air annealing has been mentioned in several reports [Don11, Sun14, Don19], but it is rarely analysed. Moreover, the superconducting properties are often studied using magnetisation measurements. This type of measurements probes the properties of the whole sample. However, it remains unclear, or even unlikely, that the annealing process changes the properties equally throughout the sample. This motivates a thorough analysis of the structural changes with respect to the sample 's surface and bulk to further our understanding of the annealing process and the resulting changes in the superconducting properties. 



\section{Experimental methods}

In this chapter, the procedure for the synthesis of $\mathrm{FeSe}_{1-x} \mathrm{Te}_{x}$ ingots and the experimental methods used to characterise the structure and magnetic properties of the extracted single crystals are introduced. First, the crystal synthesis and X-ray diffractometry (XRD) characterisation of the as-grown single crystals is presented. Thereafter, a detailed general introduction to vibrating sample magnetometers (VSM), the VSM module for the Quantum Design ${ }^{1}$ physical property measurement system (PPMS), and the specific measurement types is given. Lastly, Raman spectroscopy, scanning electron microscopy (SEM), and X-Ray photoelectron spectroscopy (XPS), which were used for the structural characterisation of the samples, are briefly introduced.

\subsection{Sample synthesis}

For the synthesis of $\mathrm{FeSe}_{1-x} \mathrm{Te}_{x}$, stoichiometric quantities of $\mathrm{Fe}$ (99.5\%), Se (99.999\%), and Te (99.9999\%) were ground, mixed, and pressed into a pellet. The pellet was then placed inside a small quartz tube, which sat inside a larger quartz tube, before being evacuated and sealed. The grinding, mixing, and pelletising of the samples were all carried out in an ultra-pure argon gas-filled glovebox. To synthesise the $\mathrm{FeSe}_{1-x} \mathrm{Te}_{x}$ single crystals, the pellet was first heated to $450^{\circ} \mathrm{C}$ at $2{ }^{\circ} \mathrm{C} / \mathrm{min}$ and kept at that temperature for 4 hours. It was then heated to $1000^{\circ} \mathrm{C}$ and dwelled for 24 hours before being slowly cooled down to room temperature at $1^{\circ} \mathrm{C} / \mathrm{min}$. This heating scheme was adapted from the literature [Sal09, Miz10, Tsu11, Mah15] and optimised according to the conditions of our furnace. A schematic diagram that illustrates the temperature-time profile of the synthesis is shown in figure 3.1 .

\footnotetext{
${ }^{1}$ Quantum Design Inc., 10307 Pacific Center Court, San Diego, CA 92121, USA.
} 


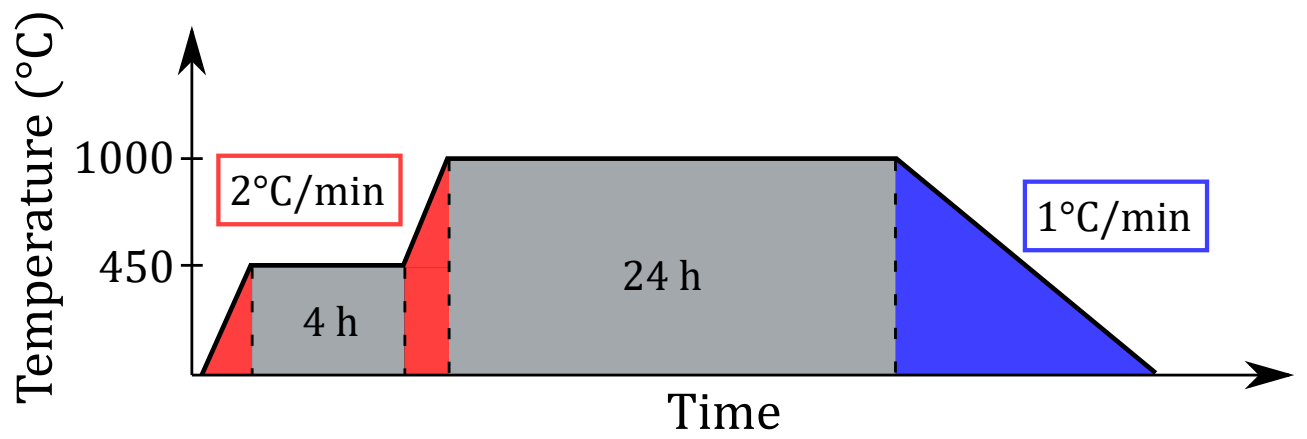

Figure 3.1: Heating scheme used for the synthesis of $\mathrm{FeSe}_{1-x} \mathrm{Te}_{x}$.

Figure 3.2 shows images of two ingots obtained following the synthesis described above. Both ingots display a shiny, reflective surface indicative of crystalline structure. From these ingots, single crystalline samples were extracted by cleaving along the ab-plane of the layered $\mathrm{FeSe}_{1-x} \mathrm{Te}_{x}$ crystal structure. The single crystals are then cut into rectangular shape for further analysis. Images of four cut single crystalline samples are depicted in figure 3.3 . In the course of this thesis, more than 100 samples have been prepared from several different $\mathrm{FeSe}_{1-x} \mathrm{Te}_{x}$ batches this way. The dimensions of the samples were measured by taking an optical microscope image and using an imaging processing software to read out the dimensions. In some cases, where the samples were too thin to be measured optically, the thickness was estimated based on the density, the measured mass, and the two other dimensions. A comparison of this procedure with the direct, optical thickness measurements of the samples where both methods could be used shows good agreement between the two methods $(\Delta c<5 \%)$.

\subsection{Annealing}

As introduced in section 2.3.3, annealing has shown promising results in improving the superconducting properties of $\mathrm{FeSe}_{1-x} \mathrm{Te}_{x}$ single crystals. For this reason, the effect of annealing is investigated in great detail in this thesis. The annealing temperature and time of the annealing process was chosen based on previous reports in the literature Noj10, Don11, Kaw12, Sun13d] and slightly adjusted based on ini- 

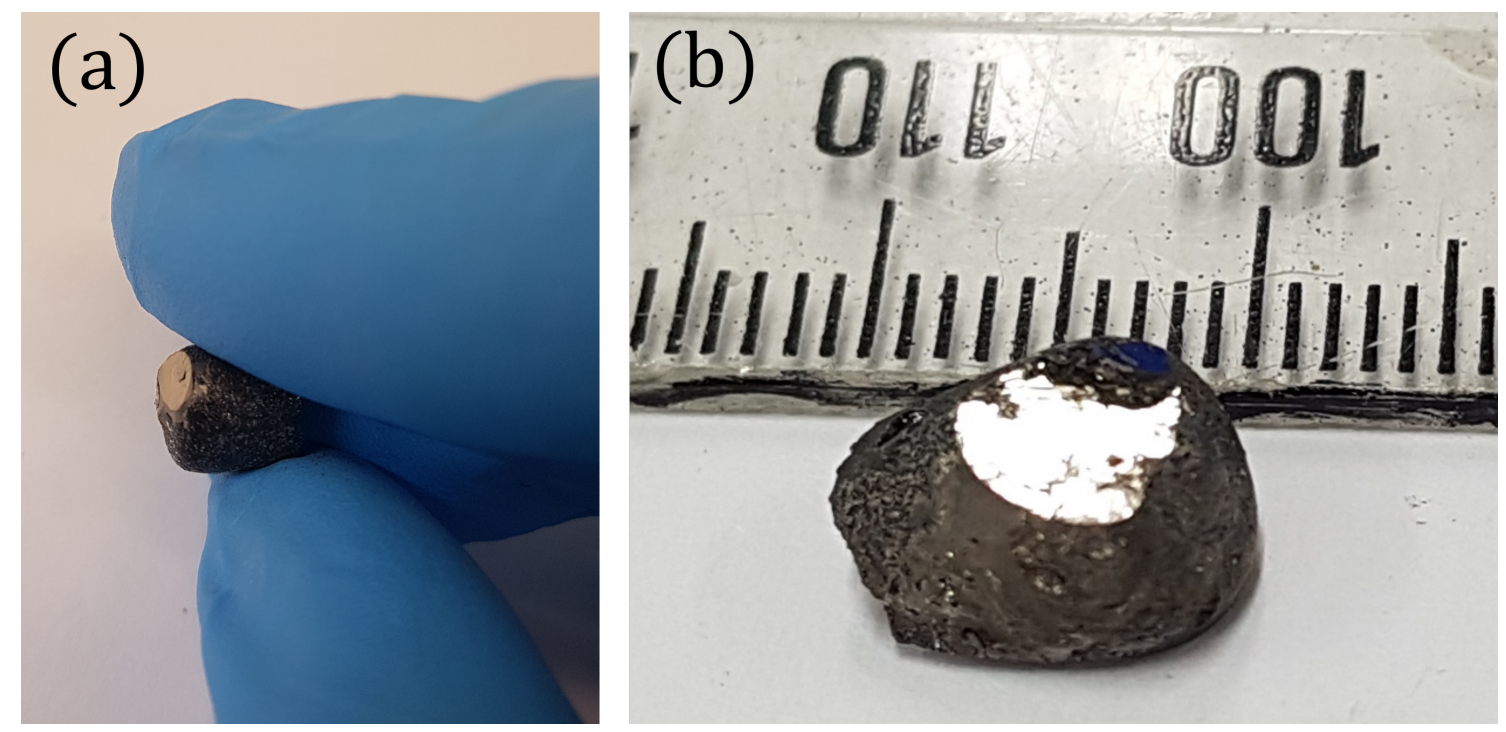

Figure 3.2: Pictures of two $\mathrm{FeSe}_{1-x} \mathrm{Te}_{x}$ ingots taken directly after the synthesis. For demonstration, a small part of the ingot has been cleaved off to show the reflective crystalline surface.
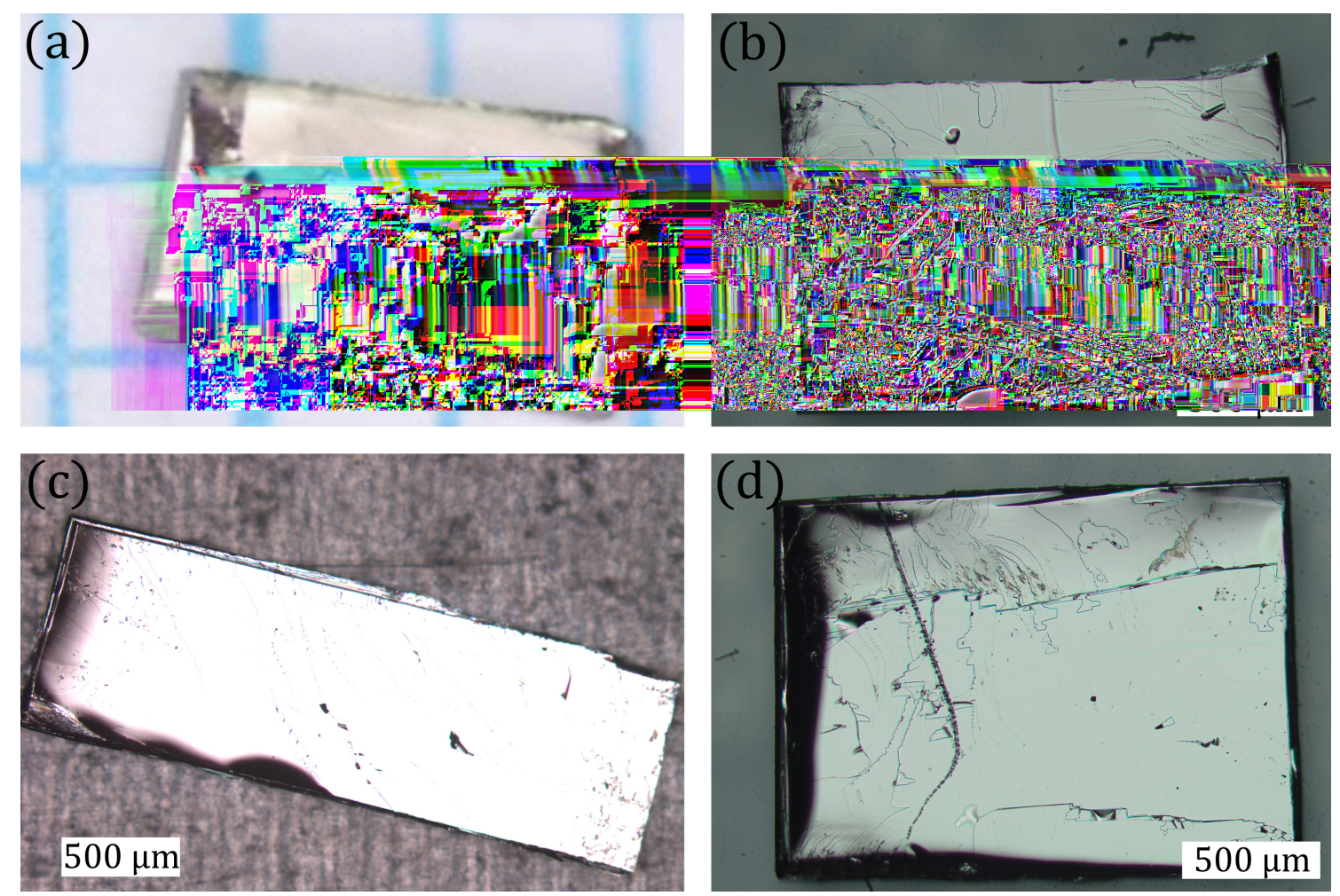

Figure 3.3: Pictures of four $\mathrm{FeSe}_{1-x} \mathrm{Te}_{x}$ single crystalline samples after they have been cut into shape. 
tial results of this work. In this context, the annealing temperature was chosen in order for the sample to not be destroyed [Sun14, but still high enough to promote diffusion and potentially chemical reactions. In regards to the annealing time, the improvement in the superconducting parameters was found to dwindle for longer annealing times and was thus chosen to be of practicable length that still induces optimal improvements. It is worth noting that the annealing temperature and time are connected in a sense that changing one of them will also change the optimal value of the other.

For the main annealing procedure, simply referred to as air annealing later in the text, a single crystal sample is placed inside a tube furnace that is continuously flushed with dry air [Boc1]. The furnace is then heated to $300^{\circ} \mathrm{C}$ within 1 hour and kept at this temperature for another 2 hours. After this, the furnace is shut off to slowly cool down.

In the case of vacuum and nitrogen annealing, further preparations steps are required. The furnace is first vacuum baked and purged several times before starting the annealing of the sample. This additional step reduces the amount of residual air and water inside the tube. After the baking and purging, the furnace is filled with the annealing atmosphere of interest for the actual annealing of the sample. Different to the previously introduced air annealing, the furnace is not continuously flushed, but sealed before the annealing to obtain a controlled nitrogen or vacuum environment.

\subsection{Vibrating sample magnetometer (VSM)}

Fundamentally, a vibrating sample magnetometer is based on electromagnetic induction. A sample is moved through a detection coil, which changes the magnetic flux in the enclosed area of the detection coil. This change in magnetic flux induces an electric field and thereby induces a measurable voltage that is proportional to the magnetic moment of the sample. 


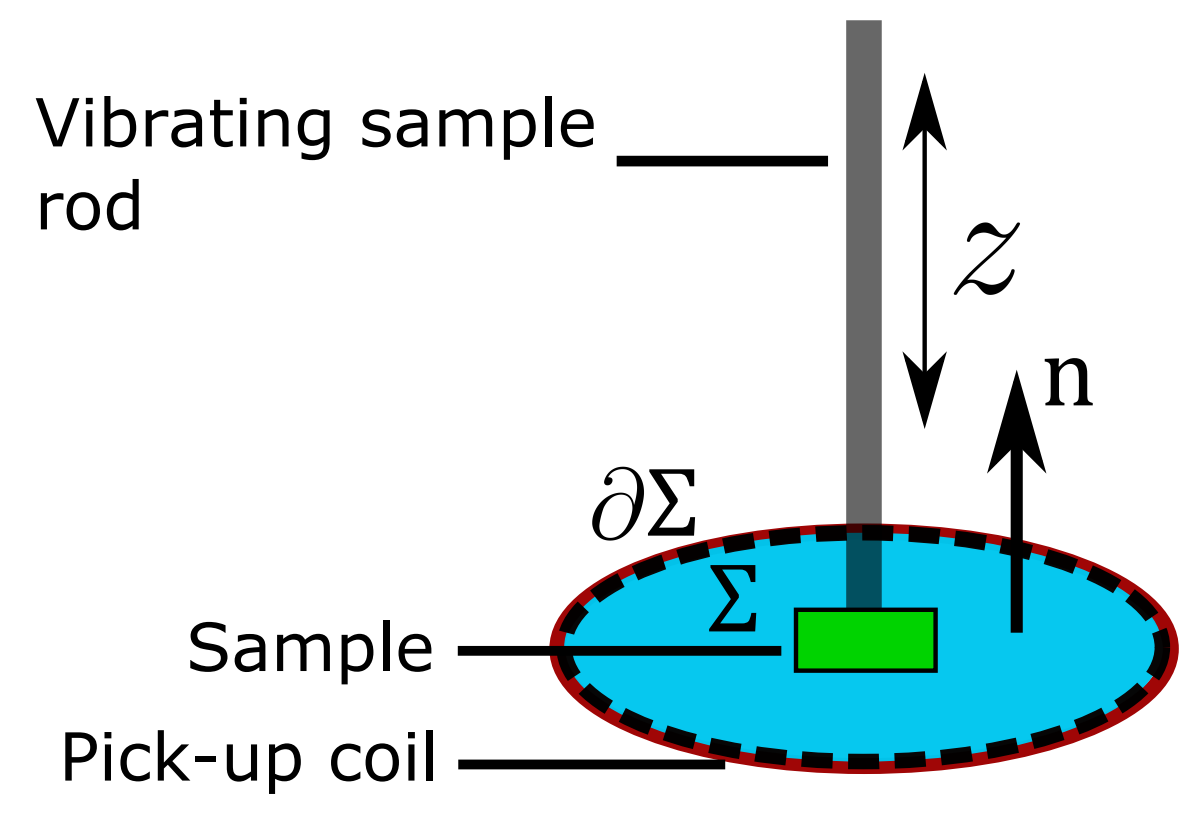

Figure 3.4: Schematic of a typical VSM setup. The area enclosed by the pick-up coil, $\Sigma$, is marked in light blue, its contour, $\partial \Sigma$, by the black dashed line, and $\boldsymbol{n}$ depicts the normal vector of the area $\Sigma$.

In general, electromagnetic induction is described by Faraday's law of induction

$$
\oint_{\partial \Sigma} \boldsymbol{E} \cdot \mathrm{d} \boldsymbol{l}=-\int_{\Sigma} \frac{\partial \boldsymbol{B}}{\partial t} \cdot \mathrm{d} \boldsymbol{A}
$$

where $\boldsymbol{E}$ is the electric field that is integrated along a contour $\partial \Sigma$ and $\partial \boldsymbol{B} / \partial t$ is the change in magnetic induction with time that is integrated over the surface $\Sigma$ [Gri08]. If one considers the basic geometry of a typical VSM setup as shown in figure 3.4 . the path of the pick-up coil of the setup can be chosen as the contour sigma. In this scenario, the area $\Sigma$ is time independent and the derivative can be put in front of the integral as such

$$
\oint_{\partial \Sigma} \boldsymbol{E} \cdot \mathrm{d} \boldsymbol{l}=-\frac{\mathrm{d}}{\mathrm{d} t} \int_{\Sigma} \boldsymbol{B} \cdot \mathrm{d} \boldsymbol{A} .
$$

Furthermore, the electric field is parallel to the contour $\partial \Sigma$ so the left side of equation 3.2 simply represents an induction voltage, $V_{\text {ind }}$. Similarly, the orientation of the relevant magnetic field is parallel to the normal of the area $\Sigma$. Therefore, the integral 
of $\boldsymbol{B}$ over the area can be rewritten as the magnetic flux $\Phi$. Equation 3.1 then simplifies to

$$
V_{\text {ind }}=-\frac{\mathrm{d} \Phi}{\mathrm{d} t}
$$

This equation can be extended by $\mathrm{d} z / \mathrm{d} z$ to obtain the following equation:

$$
V_{\text {ind }}=-\frac{\mathrm{d} \Phi}{\mathrm{d} z} \frac{\mathrm{d} z}{\mathrm{~d} t}
$$

where $z$ is the position of the sample in the $z$-direction. The term on the right, $\mathrm{d} z / \mathrm{d} t$, describes the change in position of the sample with time, which is typically set by the experimentalist. The term on the left, $\mathrm{d} \Phi / \mathrm{d} t$, mainly depends on the magnetic moment of the sample, $m$, the area enclosed by the detection coil $\Sigma$ and a coupling factor, $C$, which depends on the geometry of the detection setup and is typically calibrated using a reference sample. From this, the detected voltage of a sinusoidally oscillating sample is described by

$$
V_{\text {ind }}(t)=(C m \Sigma)(\omega \sin (\omega t))
$$

where $\omega$ is the circular frequency of the sample movement Qua08. It is worth noting that since typically an external magnetic field is applied during the measurement, this field will also contribute to the magnetic flux in the surface $\Sigma$. However, if the field is constant in time and homogenous in space, it will not induce a voltage in the detection coil.

All magnetisation measurements reported in this thesis were performed on a Quantum Design ${ }^{2}$ Physical Property Measurement system (PPMS) with VSM option. A picture of the actual experimental setup including the PPMS itself is shown in figure 3.5. This system allows for measurements at temperatures ranging from $2 \mathrm{~K}$ to $400 \mathrm{~K}$ and applied magnetic fields from $-9 \mathrm{~T}$ to $9 \mathrm{~T}$. The VSM option can resolve changes in magnetisation of less than $10^{-6} \mathrm{emu}$ at a sample rate of $1 \mathrm{~Hz}$ Qua08.

\footnotetext{
${ }^{2}$ Quantum Design Inc., 10307 Pacific Center Court, San Diego, Ca92121, USA.
} 


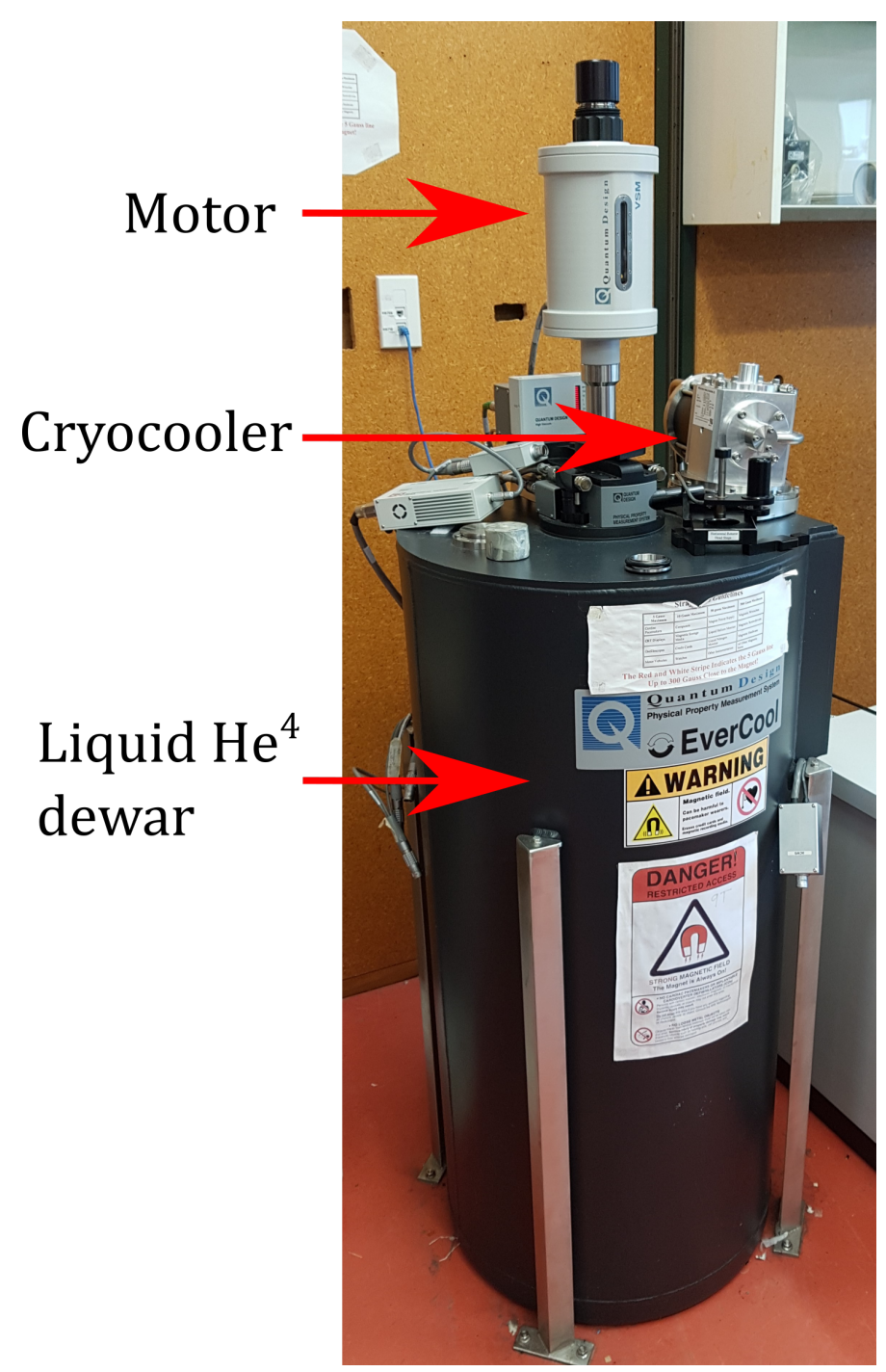

Figure 3.5: Picture of the PPMS with VSM used for all magnetisation measurements presented in this work.

As mentioned above, the measurement of the magnetic moment is based on a calibration that depends on the dimensions of the sample that is being measured. Therefore, a size-dependent correction might be necessary depending on the sample dimensions. The samples measured in this thesis are typically thinner than $1 \mathrm{~mm}$ and less than $2 \mathrm{~mm}$ in length and width. Based on the manufacturer's calibration Qua08, this leads to a deviation from the true moment of less than $0.5 \%$. This small deviation is insignificant and will be neglected for the analysis of the magnetisation in the following chapters. 


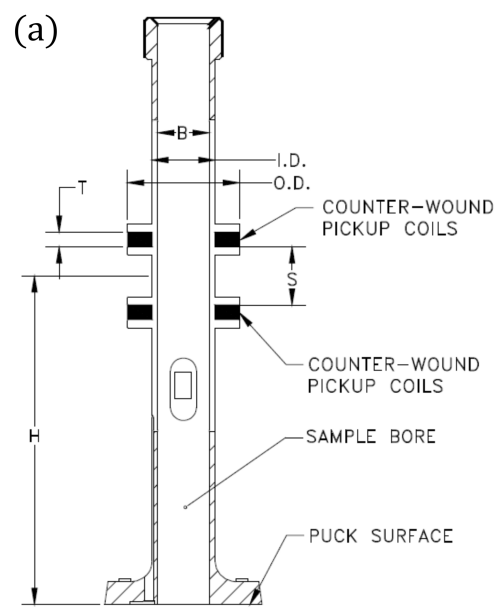

(b)

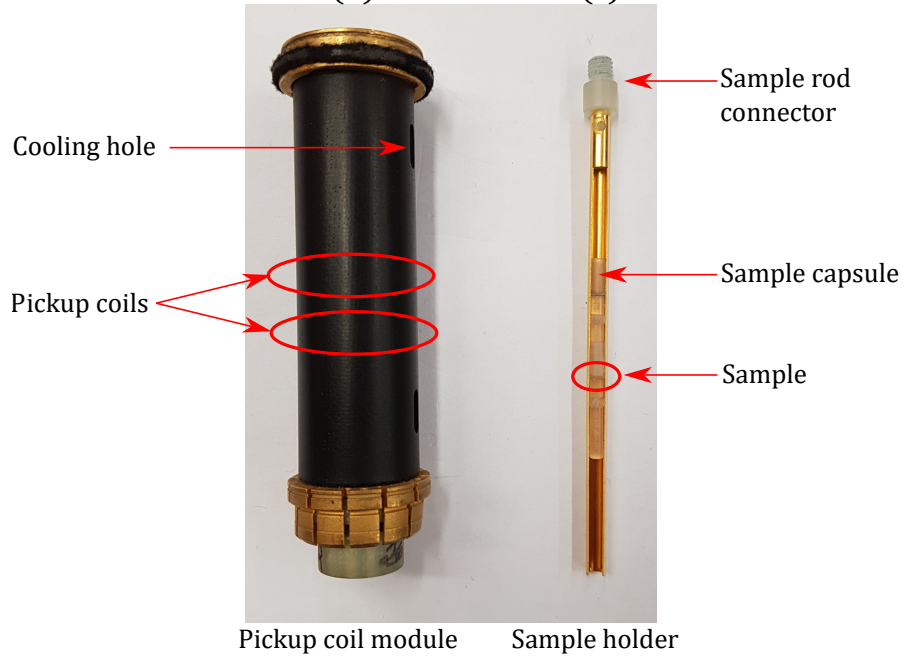

Figure 3.6: (a) Schematic drawing of the pickup coil setup (reprinted from manual Qua08). (b) Picture of the actual pickup coil module. (c) Picture of the sample holder.

Figure 3.6 shows a schematic drawing of the pickup coil (a) Qua08 and a picture of the actual pickup coil module (b) and sample holder (c). Contrary to the simple schematic to illustrate the basic working principle of a VSM introduced in figure 3.4. the pickup coil of this setup is not a single coil but a first-order gradiometer. For this first-order gradiometric setup, two coils are wound in opposite orientation to each other. Because of this, changes in flux that are homogenous in space are suppressed as they induce opposite voltages in the two coils. Such changes in flux can result from any external parasitic field or simply due to fluctuations in the applied magnetic field and would otherwise distort the measurement.

The samples itself are mounted in a small sample capsule placed inside a sample holder made of Au-coated copper. Specifically, the sample is lightly pressed between two capsules to fix it in place and prevent any movement that would otherwise jeopardise the measurements. The capsule itself is then pressed into the copper holder as shown in figure 3.6(c). The whole copper holder is connected to a sample rod, which is oscillated by the VSM motor on top of the PPMS as shown in figure 3.5 . 


\subsection{Magnetic characterisation measurements}

In this thesis, two types of magnetisation measurements were performed: temperature-dependent zero-field-cooled/field-cooled (ZFC/FC) and magnetic hysteresis loop (MHL) measurements. In the following two sections, a detailed introduction to these types of measurements and the exact experimental procedure will be given.

\subsubsection{Zero-field-cooled and field-cooled magnetisation}

Zero-field-cooled (ZFC) and field-cooled (FC) magnetisation measurements are a vital tool to investigate the properties of superconducting samples. As suggested by the name, a ZFC measurement is performed by first cooling the sample in 'zero' field and then applying a small field and measuring the magnetic moment with increasing temperature.

In this work, the sample chamber and magnet were first 'degaussed' at room temperature by applying a field of 10000 Oe and then oscillating it to 0 Oe at which the superconducting magnet was quenched. This procedure reduces the remnant field in the sample chamber to a minimum and will from now on be considered as zero field. In reality, there is still a remnant field on the order of a couple Oersted, which can be corrected for by using a second external magnetometer to measure the remnant field in the sample chamber and by applying a respective opposing field. However, in the framework of this thesis, the lowest field is at least an order of magnitude larger than the expected remnant field and therefore such corrections are not necessary.

Following the degaussing, the sample was cooled down to the lowest reachable temperature of the PPMS, $2 \mathrm{~K}$, a field of 50 Oe was applied, and the temperature was increased stepwise and stabilised at every temperature step before measuring the magnetic moment. Once a temperature that is several Kelvin above the critical temperature of the sample is reached, the sample is cooled down in the applied field to $2 \mathrm{~K}$ 


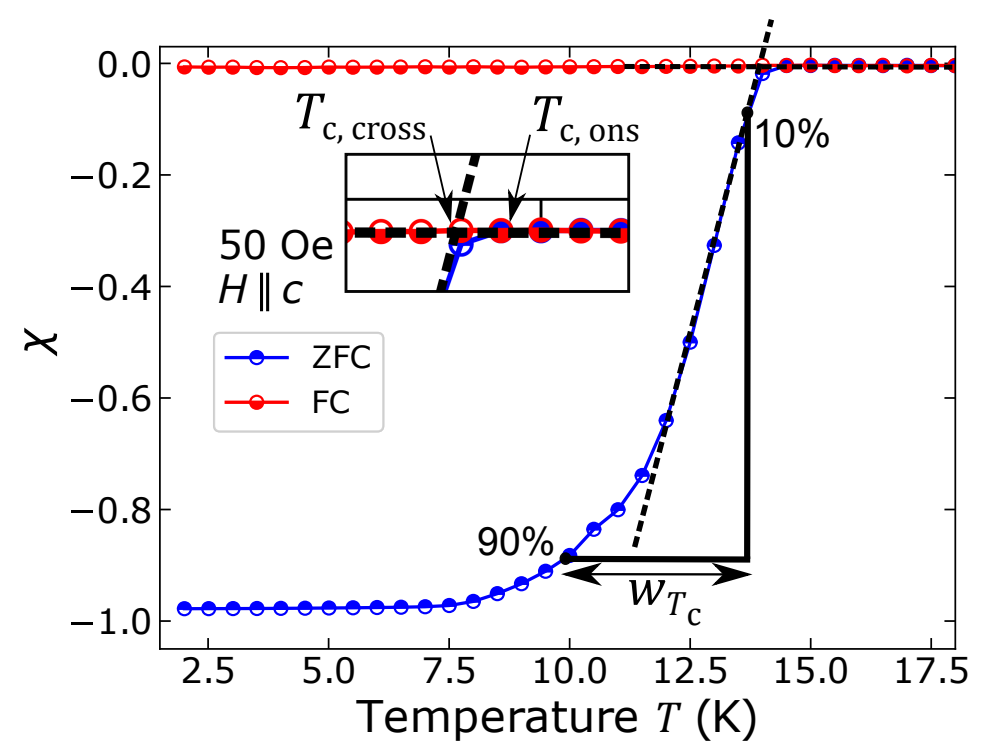

Figure 3.7: Typical ZFC (blue)-FC (red) susceptibility measurement for the estimation of the critical temperature, $T_{\mathrm{c}}$, the superconducting transition width, $w_{T \mathrm{c}}$, and the superconducting shielding fraction. $T_{\mathrm{c}, \text { ons }}$ refers to the onset- $T_{\mathrm{c}}$ and $T_{\mathrm{c}, \mathrm{cross}}$ to the crossover- $T_{\mathrm{c}}$. This measurement was performed on one of the later introduced air-annealed $\mathrm{FeSe}_{0.35} \mathrm{Te}_{0.65}$ single crystals.

(FC measurement). Again, the temperature was decreased stepwise and stabilised at every measurement temperature before measuring the magnetic moment.

Figure 3.7 shows a typical ZFC-FC magnetic susceptibility that was taken on one of the crystals synthesised in this work. From this measurement, three main characteristics of a superconductor can be extracted: the critical temperature, the superconducting transition width, and the superconducting shielding fraction.

\section{Critical temperature}

There are two prominent ways to extract the critical temperature from ZFC-FC measurements. The first is the so-called crossover- $T_{\mathrm{c}}, T_{\mathrm{c}, \mathrm{cross}}$. For this, one linear function is fitted to the steepest region of the superconducting transition of the ZFC measurement and another one to the normal state temperature-independent susceptibility for temperatures greater than $T_{\mathrm{c}}$ before the diamagnetic drop starts (see black dashed lines in figure 3.7). $T_{\mathrm{c}, \text { cross }}$ is then defined as the intersection of 
the two linear fits. The second commonly used technique is the so-called onset- $T_{\mathrm{c}}$, $T_{\mathrm{c}, \text { ons }}$. As suggested by the name, $T_{\mathrm{c}, \text { ons }}$ is defined as the temperature of the onset of the superconducting transition. In this thesis, $T_{\mathrm{c}, \text { ons }}$ was chosen to determine $T_{\mathrm{c}}$ for the following reason. First of all, $T_{\mathrm{c}, \text { ons }}$ is relatively independent of the transition width of superconducting transition in comparison to $T_{\mathrm{c}, \text { cross }}$. Secondly, the region of the transition that is to be fitted for $T_{\mathrm{c}, \text { cross }}$ is typically arbitrarily chosen and can be challenging for non-linear transitions, which technically all are.

\section{Superconducting transition width}

The superconducting transition width, $w_{T \mathrm{c}}$, is self-evidently defined as the width of the superconducting transition. As commonly estimated in the literature Che08, Kih10, Gha16, $w_{T \mathrm{c}}$ is calculated as the temperature difference between the temperature at which the magnetisation or magnetic susceptibility reaches $10 \%$ and $90 \%$ of the absolute value at the lowest measured temperature. That is if the magnetisation or magnetic susceptibility plateau at the lowest measured temperature. If the signal is not reaching a plateau at the lowest measured temperature, $w_{T c}$ can be seen as a lower boundary of the actual transition width.

\section{Superconducting shielding fraction}

Another characteristic of a superconductor is the diamagnetic response to external magnetic fields. Neglecting demagnetisation effects and assuming samples that are much larger than the magnetic penetration depth, a fully superconducting sample is expected to exhibit perfect diamagnetism $\chi=-1$ in a ZFC measurement. Perfect diamagnetism is therefore often used as a mean to quantify the quality and superconducting volume fraction of a superconducting sample [Wat07, Kit08]. However, this is typically incorrect, as a fully superconducting surface sheath will shield the bulk of the sample from the external field and thus lead to a magnetic susceptibility of -1 independent of the bulk properties of the sample. Therefore, a perfect 
diamagnetic response in the ZFC measurement only shows that the surface is perfectly superconducting, which of course is still useful information. Similarly, the ratio between the susceptibility at a temperature above $T_{\mathrm{c}}$ and the lowest measured temperature in a FC-measurements is often cited as Meissner fraction [Cam91]. However, this is only true for a perfect defect-free superconductor. For real superconducting samples, strong flux pinning typically leads to a reduction in the Meissner fraction to a value close to zero [Cam91. In that sense, the FC susceptibility is rather a measure of the pinning strength of the superconductor rather than the superconducting volume fraction.

\section{Demagnetisation correction}

It is worth noting that, depending on the sample dimensions and the orientation of the applied magnetic field, the measured magnetisation is largely influenced by demagnetisation effects. For example, a thin slab sample with the field applied perpendicular to the slab's face, which is one of the most common measurement modes and also the main mode used in this thesis, is heavily influenced by demagnetisation. For instance, demagnetisation effects might lead to what seems to be a broad superconducting transition when in fact it is a result of demagnetisation and the measured susceptibility will always be smaller than the actual susceptibility. Therefore, correct interpretation of low-field ZFC-FC measurements critically depends on the demagnetisation correction of the magnetic susceptibility, $\chi$.

For this reason, all ZFC-FC measurements presented in this thesis were demagnetisation-corrected based on the sample geometry and the numerically calculated demagnetisation factors given by Prozorov and Kogan [Pro18]. For simplicity and readability, the demagnetisation corrected susceptibility, $\chi_{\text {corr }}$, is simply referred to as $\chi$ in this thesis. 


\subsubsection{Magnetic hysteresis loop measurements}

For the magnetic hysteresis loop (MHL) measurements, the sample was first zero-field cooled to the temperature of interest. The applied field was then increased from zero field to the highest applied positive field, back to zero, increased again to the highest negative field, back to zero, and increased one more time to intermediate positive fields. Given the initial screening of the applied magnetic field in superconductors for fields below $B_{c 1}$, this last step to intermediate positive applied fields for a second time is necessary to obtain a full critical-state hysteresis loop. Unless otherwise stated, the magnetic moment was measured at stabilised applied fields for every acquired data point. This point is of high significance, as the magnitude of the measured magnetic moment of a superconductor is strongly dependent on the way it was measured (see again section 2.1.10). Additionally, measuring the MHL only with fast magnetic field sweeping rates can also prevent the discovery of interesting phenomena such as the asymmetric MHLs presented later in chapter 7.

Using equation 2.17, $J_{\mathrm{c}}$ of a sample can be estimated based of a MHL measurement and the sample dimensions. Because of this, MHL measurements are vital in the characterisation of superconductors. However, as for most measurements, it is important to remove any parasitic background signal that would otherwise falsify the $J_{\mathrm{c}}$ estimate. In this case, the critical part of the estimation is the width of the MHL, that is the difference between the magnetisation of the increasing- and decreasing applied field branch, $\Delta M=\left|M_{\text {inc }}(B)-M_{\text {dec }}(B)\right|$. To properly correct for the background, it is thus necessary to correct the background of $M_{\text {inc }}(B)$ and $M_{\mathrm{dec}}(B)$ individually. This is illustrated in figure 3.8 for a sample with a strong ferromagnetic background. First, the uncorrected, original data is split into the two branches $M_{\text {inc }}(B)$ (dark blue full circles) and $M_{\mathrm{dec}}(B)$ (light blue full circles). After this, the non-superconducting background is estimated by another MHL measurement that is performed just above the critical temperature of the sample. Similarly to the original data, the background is also split into the two branches and then 


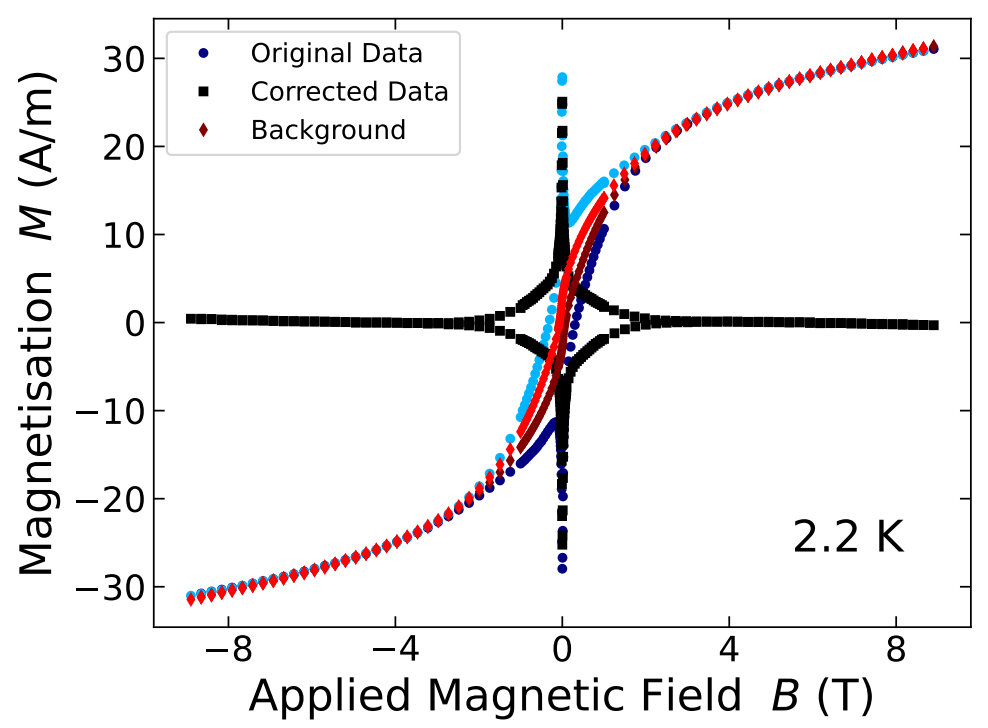

Figure 3.8: MHL of a $\mathrm{FeSe}_{0.35} \mathrm{Te}_{0.65}$ sample measured at $2.2 \mathrm{~K}$. The figure shows the original data (dark- and light blue full circles), the background (red and brown diamonds), and the background corrected data in black squares.

subtracted from the respective branch of the original data. The resulting corrected data (black solid squares) now shows the expected MHL of a superconductor without dia-, para-, or ferromagnetic background. It is worth mentioning, that for the estimate of $J_{\mathrm{c}}$, it is technically not necessary to remove the dia- or paramagnetic background since only a hysteretic background contributes to $\Delta M$. However, a full background subtraction is still useful because it typically illustrates the quality of the background correction based on the resulting superconducting hysteresis loop. In this case here, the corrected data displays a nice superconducting MHL typical of a superconductor with a low irreversibility field.

\subsection{Raman spectroscopy}

Raman spectroscopy is based on the inelastic scattering of light discovered by Raman et al. in 1928 [Ram28] (awarded a Nobel price in 1930). Intense monochromatic light is focused on the sample of interest and the scattered light is detected. Most of the photons are elastically scattered (Rayleigh scattering), but a small portion is 
inelastically scattered (Raman scattering) resulting in a energy shift of the scattered photon that depends on the vibrational states of the material. By detecting the number and energy of the scattered photons, a Raman spectrum is obtained that is characteristic to the material in question. The Raman analysis presented in this thesis was performed on a LabRam that is equipped with a $633 \mathrm{~nm}$ HeNe-laser and a notch filter to suppress the Rayleigh-line.

\subsection{Scanning electron microscopy}

Scanning electron microscopy (SEM) is based on a focused electron beam that is scanned across the sample surface of interest. In doing so, the electrons interact with the surface in various ways that can be used to infer back on the sample characteristics and properties. For this work, three types of interaction and their detection are of special interest: backscattered electron detection (BSE), the detection of X-rays that are emitted from the specimen (EDS), and the detection of transmitted electrons (STEM). In the following, a short introduction to the SEM methods relevant to this thesis is given. A more detailed introduction can be found in the comprehensive textbook on SEM by Goldstein et al. Gol18.

\subsubsection{Backscattered electron detection (BSE)}

BSE contains information on both the topography of the sample as well as the composition. This analysis is based on the detection of electrons of the original electron beam that are backscattered from the sample. One of the main characteristics of the backscattered electron signal is the atomic number dependence ( $Z$-dependence) of the backscatter cross section [Gol18]. This can be described in terms of the backscatter coefficient

$$
\eta=N_{\mathrm{BSE}} / N_{\mathrm{B}}
$$


where $N_{\mathrm{BSE}}$ is the number of backscattered electrons and $N_{\mathrm{B}}$ the number of the beam electrons. The larger the atomic number, the larger is $\eta$, and thus the more electrons get detected at the backscatter detector. The contrast of the resulting backscatter image of the probed surface can then be used to make inferences on the composition of the sample. However, for a correct $Z$-contrast analysis, the sample surface has to be finely polished as differences in the surface topography can also lead to differences in number of detected backscattered electrons.

\subsubsection{Energy-dispersive X-ray spectroscopy (EDS)}

EDS is based on the X-rays that are emitted from the specimen when an excited atom relaxes into a lower energy state and is a powerful tool to investigate the chemical composition of a sample. In short, the incident electron beam of the electron microscope leads to the ejection of electrons of the atoms of the sample. When the ejected electron is of a lower energy state than other electrons of the respective atom, one of these higher-level electrons will relax into the lower energy state. This process can lead to the emission of an X-ray that is characteristic to the electronic energy levels of the element of which is was emitted from. This technique can either be used to quantify the elemental composition of the whole probed surface or to test for inhomogeneity by scanning the surface of the sample and mapping the elemental composition of each spot individually.

\subsubsection{Scanning transmission electron microscopy (STEM)}

Scanning transmission electron microscopy is based on the detection of beam electrons that transmit through the sample. For this technique to work, the sample has to be thin enough for the electrons to pass through. In this work, this was achieved by first mechanically polishing the samples to a thin disc and then milling them further using an argon-ion mill. Similar to the $Z$-contrast in BSE, the brightness in annular dark-field STEM also depends on the atomic number of the elements present 
in the probed area [Nel08, Gol18].

All SEM measurements presented in this thesis were performed under vacuum on a FEI $]^{3}$ Nova NanoSEM 450 equipped with an BSE, EDS, and STEM detectors.

\subsection{Depth-profiling via X-ray photoelectron spectroscopy}

A powerful method to determine the chemical composition of the surface of a material is X-ray photoelectron spectroscopy. This method is based on the photoelectric effect and the energy levels of the electrons of the sample in question. When the sample is irradiated with a monochromatic X-ray beam, electrons are excited, kicked out of their atomic orbits, and their kinetic energy measured by an energy-sensitive detector. The energy and intensity of the detected electrons, that is specific to the elements present in the sample and the chemical nature of the core electrons, is then used to characterise the compound in question. For a more detailed introduction to XPS, the reader is directed to the Handbook of X-ray photoelectron spectroscopy by Moulder et al. Mou92].

The penetration depth of typical XPS X-rays is on the order of 1 to 10 micrometre Mou92]. However, the mean free path of the excited electrons in a solid is on the order of nanometres. Because of this, XPS is extremely surface sensitive only probing the top layers of a sample surface [Mou92]. In order to probe the chemical composition deeper inside the sample, XPS spectroscopy is often combined with milling techniques such as argon-ion beam milling. This way, it is possible to create depth profiles of the chemical composition of a sample.

The measurements presented in this work were performed using a Kratos 4 Axis DLD XPS spectrometer that is equipped with an argon ion gun. First, an XPS spectrum of the untreated surface was taken. Then, the surface was milled off in steps for a specified time with spectra being taken after each milling step.

\footnotetext{
${ }^{3}$ Thermo Fisher Scientific Inc., Waltham, Massachusetts, USA.

${ }^{4}$ Kratos Analytical Ltd., Trafford Wharf Road, Manchester, UK.
} 


\subsection{X-ray Diffractometry}

$\mathrm{X}$-ray diffraction (XRD) is a key tool to investigate the crystals structure of a material. The basic principle of this method is based on Bragg's law, which describes the interference condition of X-rays that are scattered from a material surface [Bra13]. This interference is either constructive or destructive depending on the crystal structure of the material, the wavelength of the material, and the angle between the incident X-ray and the sample surface. For a detailed introduction to XRD, the reader is referred to Waseda et al. [Was11.

For this thesis, two types of scans were performed: powder XRD and sample surface $\mathrm{XRD}$. The scan method employed for both types is often referred to as $2 \theta$-scan. That is, the $\mathrm{X}$-ray source and the $\mathrm{X}$-ray detector are set to the same angle in respect to the sample surface and then increased from a starting angle $\left(\theta=2.5^{\circ} \mathrm{C}\right)$ to a maximum angle $\left(\theta=45^{\circ} \mathrm{C}\right)$. In the case of powder XRD, several single crystals are ground to a powder. Assuming an optimal powder average (no preferred orientation of the finely ground crystal grains), the obtained diffractogram contains reflections of all crystalline orientations of the ground crystals. In the case of sample surface XRD, the single crystal is not ground and only the surface of the sample is scanned. Thus, only the reflections of the crystal surface can be observed. For the measurements presented in this thesis, a Bruker ${ }^{5}$ D8 was employed.

\footnotetext{
${ }^{5}$ Bruker Corporation, 40 Manning Road, Billerica, Ma 01821, USA.
} 


\section{Effect of air annealing on the structure and composition of $\mathrm{FeSe}_{1-x} \mathrm{Te}_{x}$}

To analyse and understand air annealing induced changes in the superconducting properties of $\mathrm{FeSe}_{1-x} \mathrm{Te}_{x}$, it is vital to first examine the changes in the chemical composition and structure as well as their implications. In this chapter, a comprehensive analysis of the structure of as-grown and air-annealed $\mathrm{FeSe}_{1-x} \mathrm{Te}_{x}$ single crystals is presented. First, the crystal structure of the as-grown crystals is analysed by XRD analysis. Thereafter, the structure and chemical composition of the surface of asgrown and air-annealed single crystals is analysed using optical microscopy, scanning electron microscopy (SEM), energy-dispersive X-ray spectroscopy (EDX), and Raman spectroscopy. Thereafter, the bulk properties are probed using cross-sectional Raman spectroscopy, SEM, scanning transmission electron microscopy (STEM), and depth-profiling X-ray photoelectron spectroscopy (XPS). The experimental findings are then reinforced by theoretical considerations on the oxidation process and the iron oxide layer thickness. Lastly, a diffusion model to describe the formation of the iron oxide layers and the overall annealing process is proposed.

\subsection{XRD characterisation of as-grown $\mathrm{FeSe}_{1-x} \mathrm{Te}_{x}$}

During the course of this work, several batches of differently doped $\mathrm{FeSe}_{1-x} \mathrm{Te}_{x}$ single crystals were synthesised. However, the results presented in this thesis are only based on crystals from one $\mathrm{FeSe}_{0.5} \mathrm{Te}_{0.5}$ batch, one $\mathrm{FeSe}_{0.35} \mathrm{Te}_{0.65}$ batch, and two samples from another work $\left(\mathrm{FeSe}_{0.15} \mathrm{Te}_{0.85}\right.$ and $\left.\mathrm{FeSe}_{0.65} \mathrm{Te}_{0.35}\right)$. The purity of the $\mathrm{FeSe}_{0.35} \mathrm{Te}_{0.65}$ batch and the $\mathrm{FeSe}_{0.5} \mathrm{Te}_{0.5}$ batch was checked using powder XRD. For this, several large crystals of each batch were ground for 30 minutes using mortar and piston. The powder was then placed on a quartz slide and XRD measurements were performed on 


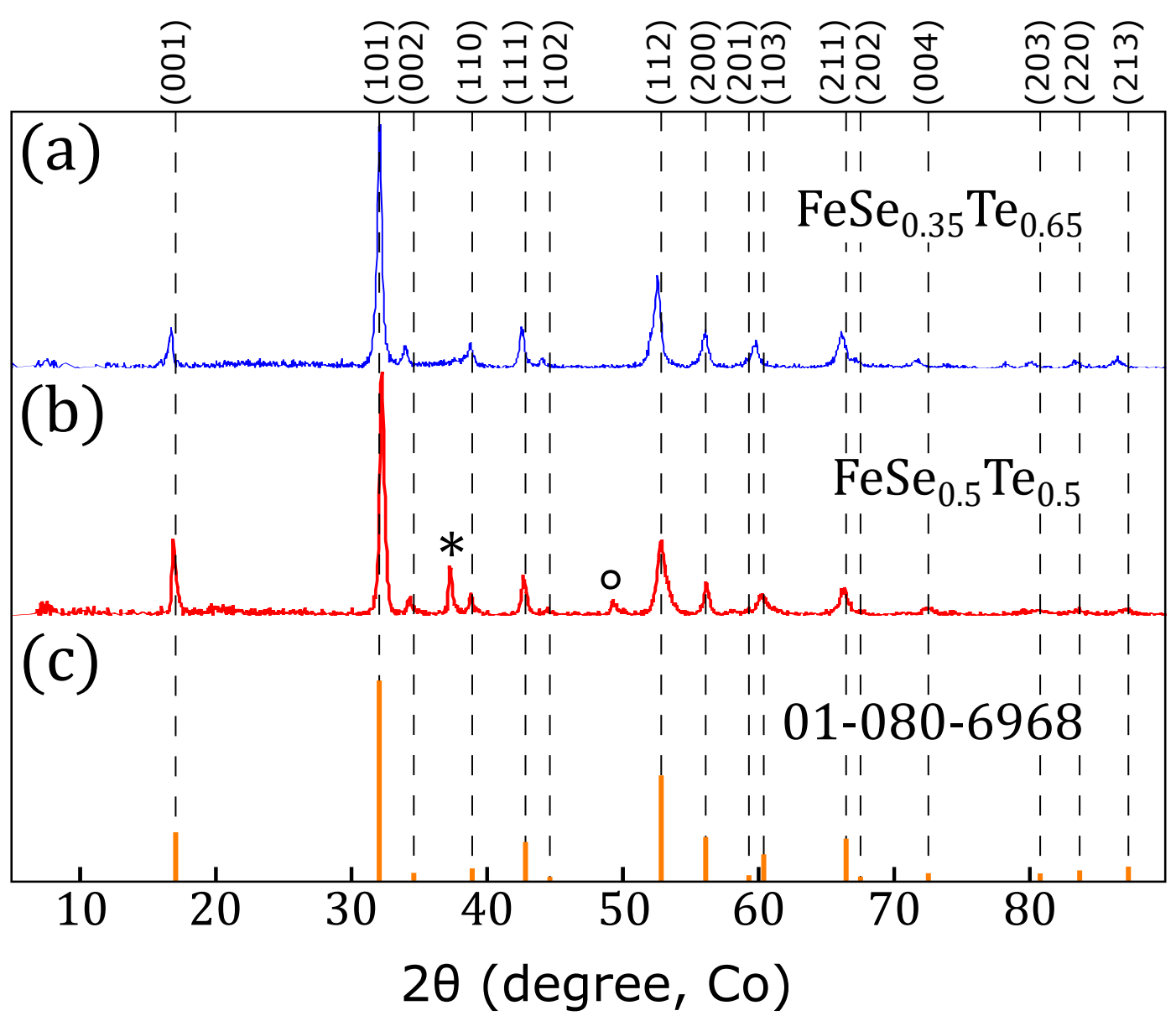

Figure 4.1: Powder XRD diffractrogram of $\mathrm{FeSe}_{0.35} \mathrm{Te}_{0.65}$ (a), $\mathrm{FeSe}_{0.5} \mathrm{Te}_{0.5}$ (b), and reference pattern 01-080-6965 $\left(\mathrm{FeSe}_{0.4} \mathrm{Te}_{0.6}\right)$. The ${ }^{*}$-reflection in (b) can be assigned to the (111) peak of $\mathrm{FeTe}_{2}$ (00-0051-1158, 01-071-6143).

the powder using a Bruker ${ }^{1} \mathrm{D} 8$ that is equipped with a cobalt anode $(\lambda=1.789 \AA)$. The crystals selected for grinding and consecutive XRD analysis were selected in the same way as any of the later analysed samples in the other sections and chapters to obtain a representative estimate of the purity.

The powder XRD diffractogram of $\mathrm{FeSe}_{0.35} \mathrm{Te}_{0.65}$ shown in figure 4.1 (a) displays sharp reflection peaks that match the reference patterns (01-084-7367, 01-080,6968) of the tetragonal P4/nmm phase of iron selenide telluride (see figure 4.1(c)). Furthermore, there are no additional reflections that would indicate the presence of additional impurity phases. The results therefore suggest a high-quality and high-purity batch

\footnotetext{
${ }^{1}$ Bruker Corporation, 40 Manning Road, Billerica, Ma 01821, USA.
} 
of single crystalline $\mathrm{FeSe}_{0.35} \mathrm{Te}_{0.65}$. Almost all results presented later in this thesis were obtained from crystals extracted from this $\mathrm{FeSe}_{0.35} \mathrm{Te}_{0.65}$ batch.

Overall, the diffractogram of the $\mathrm{FeSe}_{0.5} \mathrm{Te}_{0.5}$ batch shown in figure 4.1(b) is fairly similar to that of $\mathrm{FeSe}_{0.35} \mathrm{Te}_{0.65}$. The reflections are slightly shifted due to the change in doping and the resulting change in the lattice parameters, but the main reflection peaks are still sharp and match with the reference patterns for the superconducting, tetragonal $\mathrm{P} 4 / \mathrm{nmm}$ phase. However, there are two small impurity reflection peaks. The first impurity peak at $2 \theta=37.3^{\circ}$ can be indexed to the $\mathrm{FeTe}_{2}$ (111)-peak (000051-1158, 01-071-6143). The origin of the second impurity peak at $49.3^{\circ}$ is unknown. Based on the size of the $\mathrm{FeSe}_{1-x} \mathrm{Te}_{x}$ reflections in relation to the impurity reflections, the $\mathrm{FeSe}_{1-x} \mathrm{Te}_{x}$ phase is still dominating overall. The $\mathrm{FeTe}_{2}$ impurity phase and the other impurity phase are most likely part of the flux that remained on some of the crystals of this batch. This is reinforced by the single crystal surface XRD of a flux-free crystal that did not exhibit any $\mathrm{FeTe}_{2}$ reflections (see figure 4.2(c)). For this reason, extra care was taken in selecting a crystal with no flux for the magnetic analysis presented later in chapter 5.

Rietveld refinement was performed on both powder XRD diffractograms using the FullProf suite Rod93]. In both cases, the amplitudes of the different reflections were found to slightly deviate from the reference patterns. More specifically, the (001) reflections were larger than expected. This is not too surprising since the amplitude of the different reflections of a powder diffractrogram are based on a perfect powder average: all grains are randomly orientated without any orientational preferences. Since both powder samples were obtained by picking and grinding small single crystals that are naturally $c$-axis oriented, perfect powder average was not achieved. Instead, the (001) reflections are slightly overrepresented. Nevertheless, the lattice parameters are determined by the position of the different reflections and can therefore still be accurately estimated. The resulting estimates for the lattice parameters of the $\mathrm{FeSe}_{0.35} \mathrm{Te}_{0.65}$ and $\mathrm{FeSe}_{0.5} \mathrm{Te}_{0.5}$ batch are summarised in table 4.1. The $a$ and $c$ lattice parameter of both batches generally match the lattice 
Table 4.1: Lattice parameter of $\mathrm{FeSe}_{1-x} \mathrm{Te}_{x}$ derived from powder and single crystal surface XRD of this work and the literature [Sal09, Tsu11, Sun16, Tae09].

\begin{tabular}{l|l|l|l|l} 
& \multicolumn{2}{|l|}{ Powder XRD } & \multicolumn{2}{l}{ Single Crystal Surface } \\
& $a(\AA)$ & $c(\AA)$ & $a(\AA)$ & $c(\AA)$ \\
\hline $\mathrm{FeSe}_{0.15} \mathrm{Te}_{0.85}$ & $3.8047(5)$ & $6.112(1)$ & & 6.217 \\
$\mathrm{FeSe}_{0.35} \mathrm{Te}_{0.65}$ & $3.8064(7)$ & $6.052(2)$ & & 6.084 \\
$\mathrm{FeSe}_{0.5} \mathrm{Te}_{0.5}$ & & & 6.050 \\
$\mathrm{FeSe}_{0.65} \mathrm{Te}_{0.35}$ & 3.815 & 6.248 & & \\
\hline $\mathrm{FeSe}_{0.1} \mathrm{Te}_{0.9}[$ Sal09] & 3.810 & 6.165 & & \\
$\mathrm{FeSe}_{0.25} \mathrm{Te}_{0.75}[$ Sal09] & 3.803 & 6.118 & & \\
$\mathrm{FeSe}_{0.33} \mathrm{Te}_{0.67}[$ Sal09] & 3.815 & 6.069 & & \\
$\mathrm{FeSe}_{0.5} \mathrm{Te}_{0.5}[$ Sal09] & 3.8011 & 6.0409 & & \\
\hline $\mathrm{FeSe}_{0.5} \mathrm{Te}_{0.5}[$ Tsu11] & & & & 6.239 \\
\hline $\mathrm{FeSe}_{0.12} \mathrm{Te}_{0.88}[$ Sun16] & & & & 6.125 \\
$\mathrm{FeSe}_{0.33} \mathrm{Te}_{0.67}[$ Sun16] & & & & 6.068 \\
$\mathrm{FeSe}_{0.43} \mathrm{Te}_{0.57}[$ Sun16] & & & &
\end{tabular}

parameters of similarly doped samples in the literature [Sal09, Tsu11, Sun16]. The slight variations between different reports and this work result from small doping differences and differences in the amount of interstitial excess iron. This is best illustrated by the lattice parameters reported by Taen et al. for the as-grown state and after annealing [Tae09]. After annealing, the excess iron concentration is reduced and so is the $c$ lattice parameter. Lastly, it is worth noting, that the difference in doping mostly manifests in a change in the $c$ lattice parameter; $a$ remains mostly unaffected.

Figure 4.2 shows XRD diffractograms of the surface of whole, as-grown $\mathrm{FeSe}_{1-x} \mathrm{Te}_{x}$ crystals for $\mathrm{x}=0.35, \mathrm{x}=0.5, \mathrm{x}=0.65$ and $\mathrm{x}=0.85$. The diffractograms of all dopings display four sharp (001) reflection peaks. This suggests a high degree of crystallinity and that the crystal structure of the samples is nicely aligned with the $c$-axis of the crystals. Of all dopings, only $\mathrm{FeSe}_{0.5} \mathrm{Te}_{0.5}$ exhibits one additional reflection peak marked with *. This reflection matches the (101) reflection of $\mathrm{FeSe}_{0.5} \mathrm{Te}_{0.5}$. Therefore, it is likely that this reflection results from a layer that was exfoliated due to the weak, 


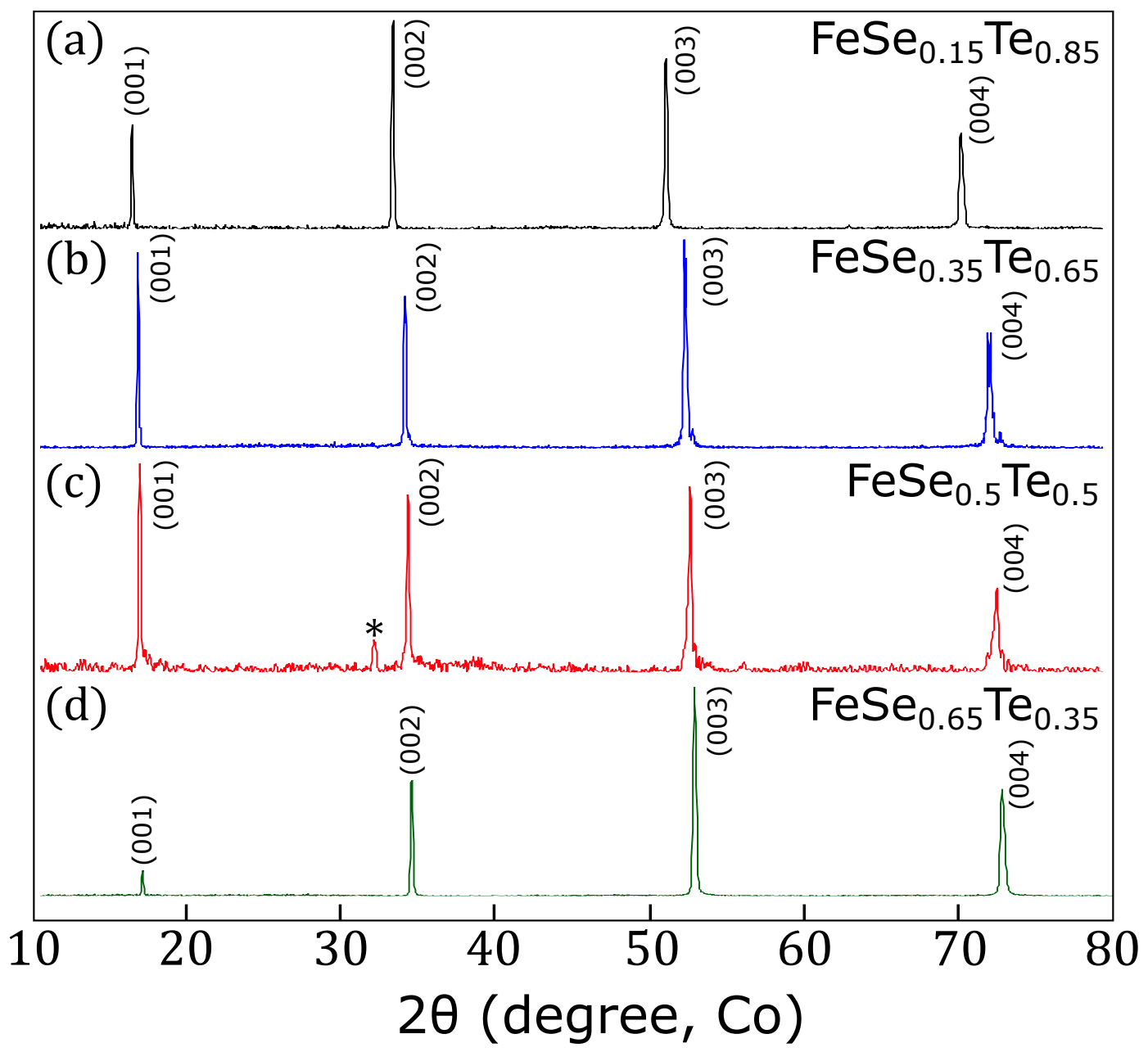

Figure 4.2: XRD diffractograms of the surface of whole, as-grown $\mathrm{FeSe}_{1-x} \mathrm{Te}_{x}$ single crystals. All four diffractograms display four sharp (001) reflections. The *-reflection of the $\mathrm{FeSe}_{0.5} \mathrm{Te}_{0.5}$ sample can be matched with the (101) peak of $\mathrm{FeSe}_{0.5} \mathrm{Te}_{0.5}$.

layered structure of this compound and rotated to match the (101) orientation.

The position of the reflection peaks slightly shifts with doping: the higher the selenium concentration, the higher the reflection angle. Since the (001) reflection positions are inversely related to the $c$ lattice parameter, this indicates a decrease in $c$ with increasing selenium concentration. This is expected, since selenium and tellurium occupy the same lattice position and the ionic radius of selenium is smaller than that of tellurium. The actual estimates of the $c$ lattice parameter from the single crystal surface XRD are summarised in table 4.1 together with the powder $\mathrm{XRD}$ results and reports from the literature. Overall, the $c$ lattice parameters from 

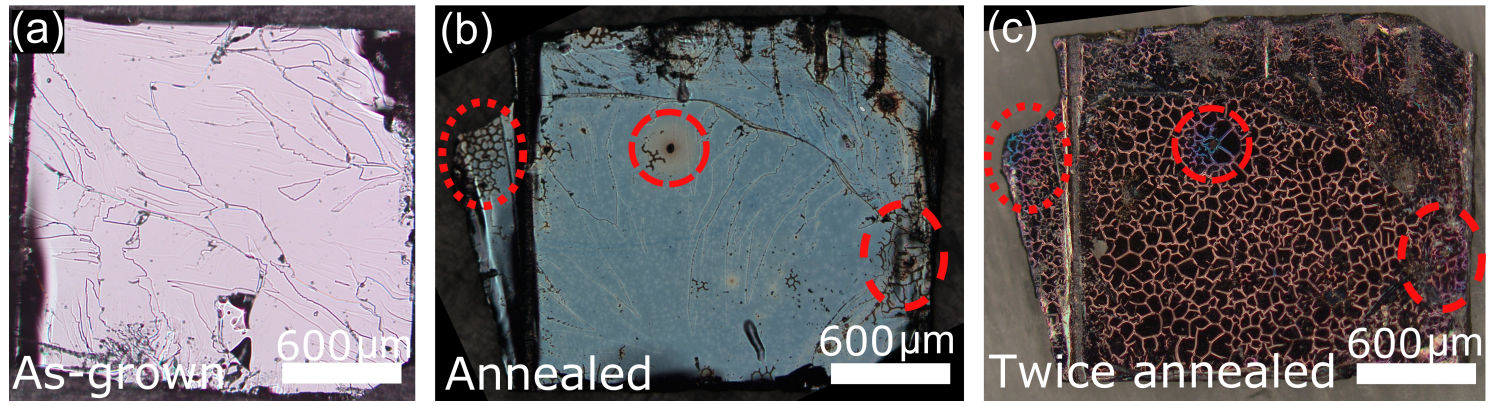

Figure 4.3: Optical microscope images of a thin as-grown $\mathrm{FeSe}_{0.35} \mathrm{Te}_{0.65}$ sample (a), after single annealing (b), and after annealing twice (c). After the first annealing, the surface turned light blue and small peculiar web-like features started to form on some parts of the sample (marked by red circles). After annealing for a second time, the entire surface of the sample was covered by the web-like structures. Interestingly, these features were already visible around defect sites after the first annealing (dashed circles in (b)), but grew denser and changed colour with further annealing.

this work match the results from similarly doped samples reported in the literature and follow the same selenium-concentration-dependence. There are some slight deviations, but as pointed out by Taen et al. [Tae09], there are always slight variations in the doping and lattice parameter of different crystals from the same batch. Therefore, small variations are inevitable. These variations within a batch are also likely to have led to the small difference between the $c$ lattice parameter from the powderand single crystal XRD of $\mathrm{FeSe}_{0.35} \mathrm{Te}_{0.65}$ from this work.

\subsection{Optical microscopy and SEM-EDS}

Figure 4.3 shows optical microscopy images of a thin as-grown $\mathrm{FeSe}_{0.35} \mathrm{Te}_{0.65}$ sample (a), after air annealing once (b), and after air annealing twice (c). The surface of the as-grown sample is black and reflective as is typically observed for single crystals of this compound [Sal09, Vie10, Mah15, Sun14] and other iron-based superconductors such as $\mathrm{BaFe}_{2} \mathrm{As}_{2}$ [Rei14]. After the first annealing, the colour of the surface changed from black to light blue. This change in colour has been observed for all air-annealed samples prepared in this work and is commonly reported for this family of superconductors [Don11, Sun14, Don19]. Additionally, web-like structures, that have not 

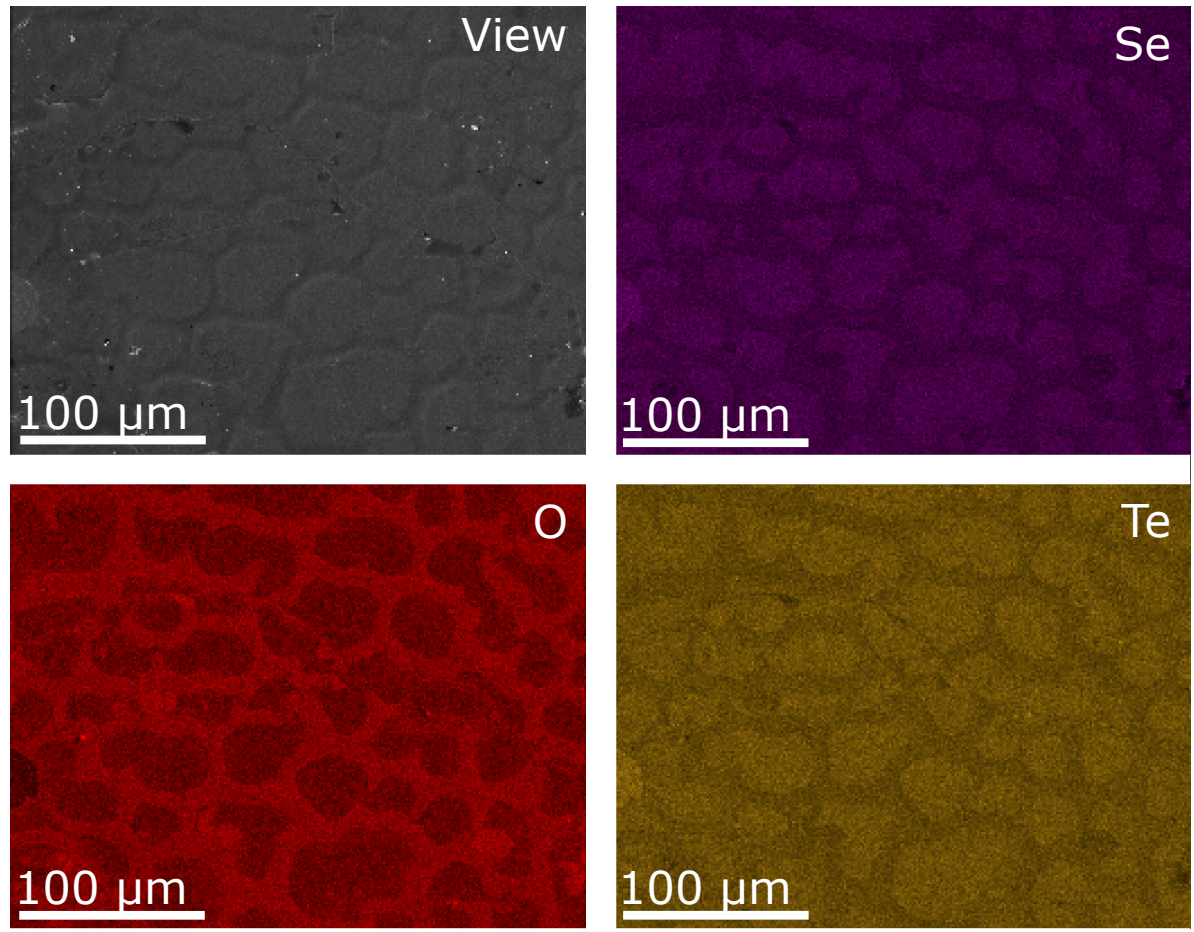

Figure 4.4: EDS mapping of the twice annealed $\mathrm{FeSe}_{0.35} \mathrm{Te}_{0.65}$ sample shown in figure 4.3(c). The figure shows the field of view (View) and the elemental EDS mapping for tellurium $(\mathrm{Te})$, selenium $(\mathrm{Se})$, and oxygen $(\mathrm{O})$.

been reported in the literature before [Don11, Sun14, Don19], formed on the surface of some samples such as the air-annealed sample shown in figure 4.3(b), where the web-like structures started to form in some areas of the sample marked with red circles. After further annealing, the web-like structures continued to spread covering the whole sample as shown in figure 4.3(c). Interestingly, the web-free surface changed back to a black colour similar to the surface of the as-grown sample.

There is strong evidence to suggest that the formation of these web-structures on the surface depends on the annealing time, the sample thickness, and the presence of defect sites. The latter two are best illustrated in figure 4.3(b), where the weblike structure already started to appear around defect sites (red dashed circles) and a thin layer on the side (red dotted circle) after the first annealing whilst the remaining surface of the sample was still free of this web-like structure. Annealing for a second time was necessary for the propagation of the web-like structure over the entire surface of the sample with the web-like structure in the circled areas changing colour 


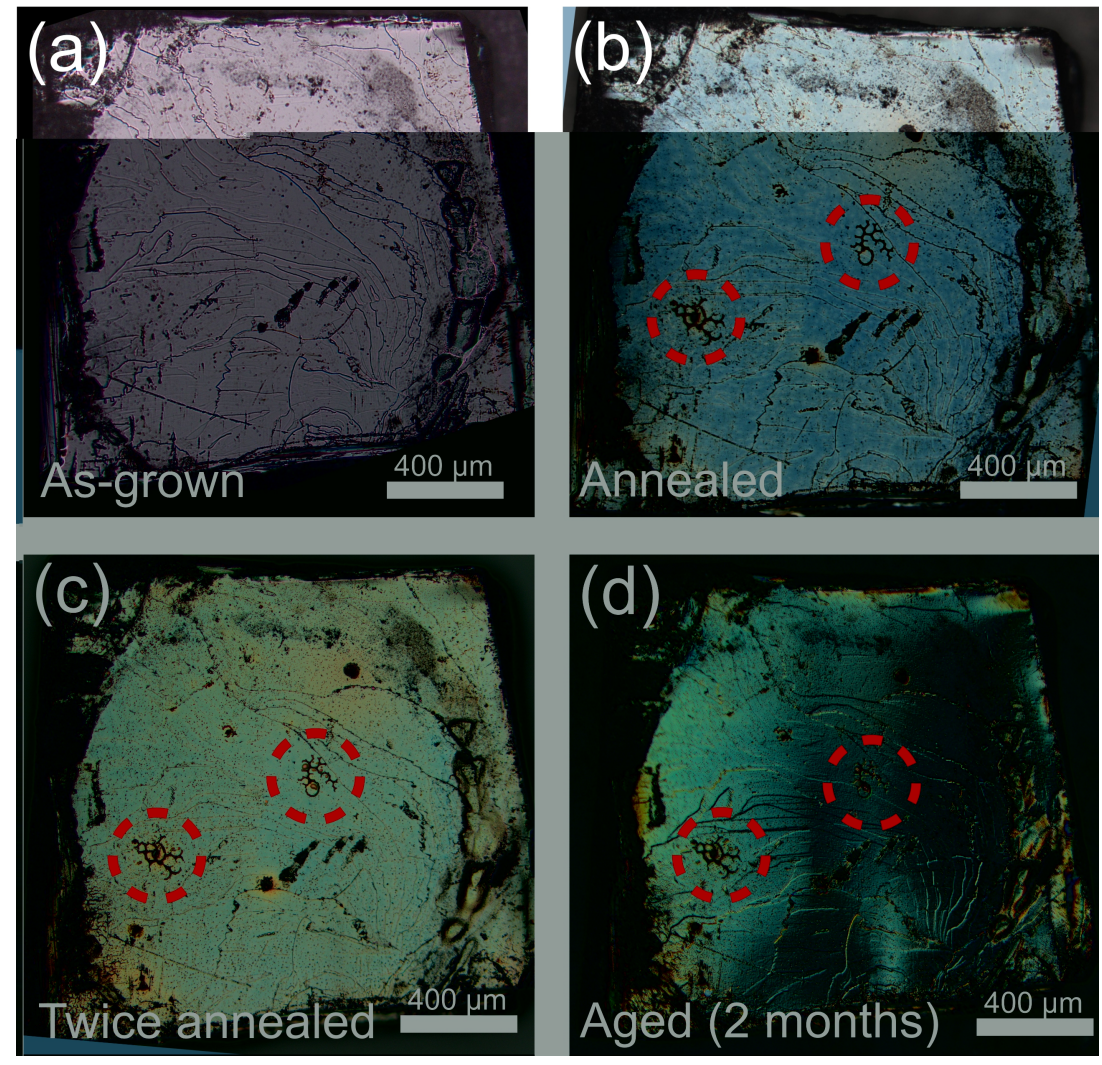

Figure 4.5: Optical microscope images of a thick as-grown $\mathrm{FeSe}_{0.35} \mathrm{Te}_{0.65}$ sample (a), after annealing once (b), after annealing twice (c), and after an additional two months of aging (d). After the first annealing, the surface turned light blue and small peculiar weblike features started to form on some parts of the sample (marked by red circles). After annealing for a second time the web-like structure did not propagate on the whole surface. Even after two months of aging, the web-like structure remained unchanged.

from a golden colour to shades of purple (see figure 4.3(c)).

Figure 4.4 shows the SEM field of view (View) and three elemental EDS mappings for tellurium (Te), selenium (Se), and oxygen (O) of a section of the twice annealed sample of figure 4.3 (c). The oxygen mapping indicates that the whole surface contains oxygen with the web-like structure itself having a higher oxygen concentration in comparison to the rest of the sample. This is vividly demonstrated by the pronounced web-like pattern in the oxygen map and its similarity to the pattern seen in the field of view. On the other hand, the selenium and tellurium concentration is lower in the web-like structure compared to the surrounding surface. The two findings together therefore suggest that the web-like structure consists of iron oxide 

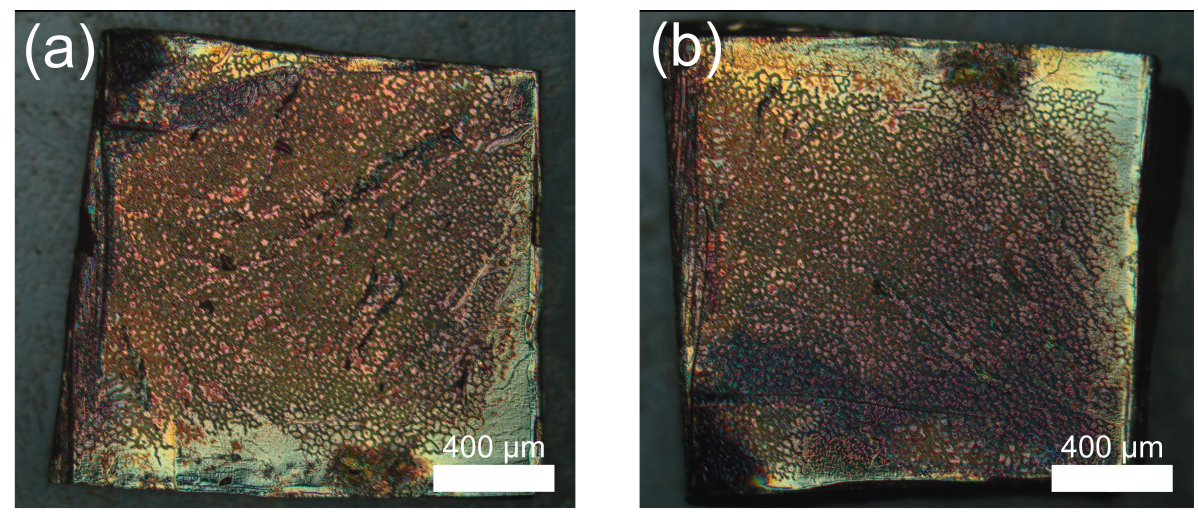

Figure 4.6: Optical microscope images of two inner surfaces of the cleaved, thick, aged sample (figure 4.5(d)). Both inner surfaces are almost fully covered by the web-like structure. The web-structure-free surfaces are light blue.

species.

In addition to the thin sample shown in figure 4.3, a sample with identical length and width, but eight times the thickness, which has been cleaved off from the same crystal as the sample shown in figure 4.3, has been analysed. Similar to the thin sample, annealing of the thick sample has also led to the formation of a blue surface layer and web-like structures around defect sites (see figure 4.5(b)). However, the web structure did not fully propagate throughout the whole surface of the sample after annealing for a second time (c) or an additional 2 months of aging (d). This again reinforces that the sample thickness plays a critical part in the formation of the surface oxides.

The analysis presented thus far has been focused on the effect of annealing on the surface of the investigated samples. However, the question arises if this oxidation process only takes place on the surface of the sample or if it might in fact also take place in the bulk of the sample. By coincidence, whilst picking up the aged, thick sample shown in figure 4.5(d), it broke into two pieces as if it had been nicely cleaved along the ab-plane. The two inner surfaces of this accidentally 'cleaved' $\mathrm{FeSe}_{0.35} \mathrm{Te}_{0.65}$ sample are shown in figure 4.6. Both surfaces are almost completely covered by the web-like structure with the web-structure-free surface being light blue and reflective. This observation is intriguing, as it shows that the inner part of a sample can also 

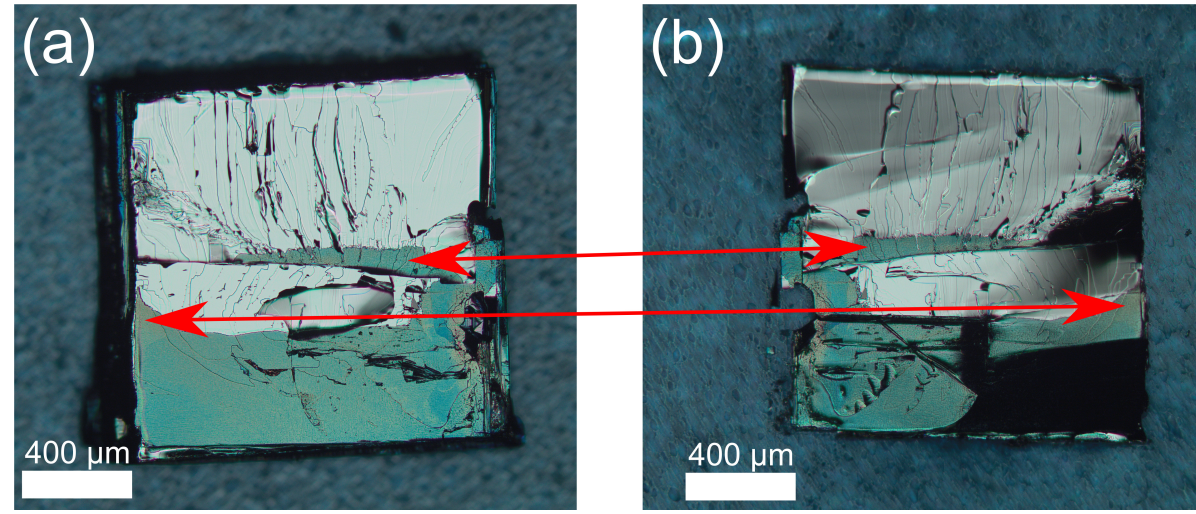

Figure 4.7: Optical microscope images of the two inner surfaces of a cleaved, air-annealed $\mathrm{FeSe}_{0.35} \mathrm{Te}_{0.65}$ sample. Both surfaces are partially oxidised: The bottom half is oxidised, but the top is not. Furthermore, both surfaces are oxidised symmetrically as indicated by the red arrows.

oxidise under certain conditions due to the weakly-bound, layered crystal structure of $\mathrm{FeSe}_{1-x} \mathrm{Te}_{x}$ (see section 2.3.1 in the theoretical background chapter). The synthesis and post-processing of the samples inevitably lead to small microcracks or defects that allow oxygen to diffuse into the sample between the weakly-bound layers using the gap created by the defects. Consequently, the oxygen present at the interface of the exposed inner surfaces during annealing reacts with the surface iron of the sample analogous to the oxidation on the outer surfaces.

The formation of iron oxides inside the sample has not only been observed for the sample shown in figure 4.6, but was observed in several annealed samples with varying levels of oxidation. This is illustrated by the two matching inner surfaces of another cleaved, air-annealed sample shown in figure 4.7 (regions of symmetry are marked with red arrows). In this case, only about half the sample's surface is oxidised: The lower part of both surfaces is oxidised, but the top part remains oxygen free. This insinuates that the oxygen diffused in through a gap-defect on the bottom side of the sample, forming iron oxides on both surfaces. However, the top part was still too tightly bound for the oxygen to diffuse through.

The above analysis shows that not only the outer surfaces of $\mathrm{FeSe}_{1-x} \mathrm{Te}_{x}$ oxidise during air annealing, but that exposed inner surfaces can also oxidise whenever 

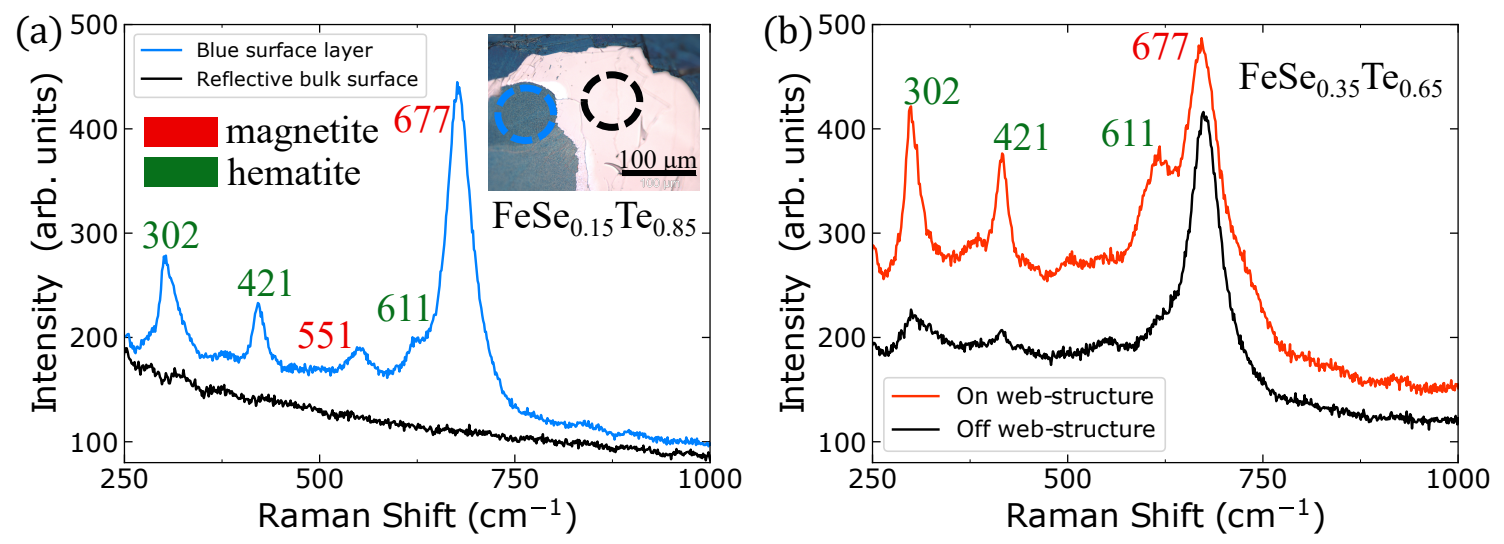

Figure 4.8: (a) Raman spectra of the blue surface layer (blue curve) and the reflective bulk surface beneath (black curve) of an air-annealed $\mathrm{FeSe}_{0.15} \mathrm{Te}_{0.85}$ single crystal at room temperature. The optical microscope picture in the inset shows the region where the spectra for the blue surface layer (blue dashed circle) and the bulk beneath (black dashed circle) were taken. (b) Raman spectra of the twice annealed $\mathrm{FeSe}_{0.35} \mathrm{Te}_{0.65}$ sample of figure 4.3 (c) for the web-structure and off the web-structure.

oxygen is able to diffuse into the sample via defects or gaps. As illustrated by figure 4.6 and 4.7 , the extend of the oxidation of the inner surfaces can vary depending on the extend of the gap. However, it is important to note that even though iron oxide is found to form in the inner region of the sample, it still remains to be shown if this only applies to exposed surfaces including those caused by defects or if the inner bulk of the sample itself is being oxidised at the same time.

\subsection{Raman spectroscopy}

The inset of figure 4.8(a) shows an optical microscope picture of an air-annealed $\mathrm{FeSe}_{0.15} \mathrm{Te}_{0.85}$ single crystal. Similar to the $\mathrm{FeSe}_{0.35} \mathrm{Te}_{0.65}$ samples discussed above, a blue layer formed on the surface of the $\mathrm{FeSe}_{0.15} \mathrm{Te}_{0.85}$ sample after air annealing. To investigate the chemical composition of the blue surface layer in comparison to the bulk beneath, a part of the blue surface layer was removed and Raman spectra were taken within the respective circled areas shown in the inset of figure 4.8(a). It is worth noting that even though the microscope was focused on the blue surface layer, the bulk surface beneath is still in focus. Given the depth of field of the microscope 
at this magnification, this insinuates an upper limit of the iron oxide layer thickness on the order of several micrometers.

Figure 4.8(a) shows the Raman spectra of the blue surface layer (blue curve) and the bulk beneath (black curve) for the air-annealed $\mathrm{FeSe}_{0.15} \mathrm{Te}_{0.85}$ single crystal taken at room temperature. For the blue surface layer, two sets of characteristic Raman peaks at $302 \mathrm{~cm}^{-1}, 421 \mathrm{~cm}^{-1}, 611 \mathrm{~cm}^{-1}$ and at $551 \mathrm{~cm}^{-1}, 677 \mathrm{~cm}^{-1}$ are observed. These Raman modes can be assigned to hematite $\left(\alpha-\mathrm{Fe}_{2} \mathrm{O}_{3}\right)$ and magnetite $\left(\mathrm{Fe}_{3} \mathrm{O}_{4}\right)$, respectively [DeF97, She03, Cha05, Pra16]. The Raman modes of $\mathrm{FeSe}_{1-x} \mathrm{Te}_{x}$ are expected below $300 \mathrm{~cm}^{-1}$ [Xia09, Oka11] and therefore were not observed in the measured range. A summary of the Raman modes is given in table 4.2 .

Contrary to the spectrum of the blue surface, the spectrum of the surface beneath the blue layer shows no Raman modes in the measured range (neither hematite nor magnetite). This shows that air annealing only led to the formation of magnetite and hematite on the surface of the sample with the bulk beneath being free of iron oxide. However, it should be noted that the blue layer might also contain other compounds that appear outside of the measurement range. For instance, the formation of $\mathrm{FeTe}_{2}$, which Raman modes appear below $200 \mathrm{~cm}^{-1}$ [Lut91, was observed by Tamegai et al. for oxygen-annealed $\mathrm{FeSe}_{0.4} \mathrm{Te}_{0.6}$ samples [Tam16].

Figure 4.8(b) shows the Raman spectra of the previously introduced twice annealed $\mathrm{FeSe}_{0.35} \mathrm{Te}_{0.65}$ sample (see figure 4.3(c)) for the web-structure and the surrounding

Table 4.2: Raman modes of magnetite [DeF97, hematite [DeF97, $\mathrm{FeSe}_{0.3} \mathrm{Te}_{0.7}$ [Xia09, and $\mathrm{FeSe}_{0.4} \mathrm{Te}_{0.6}$ Oka11 from the literature in comparison to the blue surface layer of figure $4.8(\mathrm{a})$.

\begin{tabular}{r|c|c|c|c|c|c|c} 
Compound & \multicolumn{7}{|c}{ Wavenumber $\left(\mathrm{cm}^{-1}\right)$} \\
\hline Magnetite [DeF97] & & & & & & 550 & 677 \\
\hline Hematite [DeF97] & & & 299 & 410 & 612 & & \\
\hline $\mathrm{FeSe}_{0.3} \mathrm{Te}_{0.7}[$ Xia09] & 159 & 196 & & & & & \\
\hline FeSe $_{0.4} \mathrm{Te}_{0.6}$ [Oka11] & 158 & 202 & & & & & \\
\hline Blue surface layer & & & 302 & 421 & 611 & 551 & 677
\end{tabular}


surfaces. Both the web-structure and the surrounding surfaces contain magnetite and hematite, similar to that observed in figure 4.8(a) for $\mathrm{FeSe}_{0.15} \mathrm{Te}_{0.85}$. However, the Raman spectrum of the surrounding web-structure-free surfaces exhibits predominately magnetite modes and only weak hematite peaks in comparison to the spectrum taken on the web-like structure. This insinuates larger amounts of hematite, where the iron is in a higher oxidation state $\left(\mathrm{Fe}^{3+}\right.$ versus $\mathrm{Fe}^{2+}$ and $\mathrm{Fe}^{3+}$ in magnetite), for the web structure compared to the surrounding surfaces. This result aligns well with the EDS analysis in figure 4.4, that shows a higher oxygen concentration for the web-like structure. It is worth noting, the web-structure spectrum (figure 4.8(b), red curve) also exhibits a small shoulder peak to the right of the $677 \mathrm{~cm}^{-1}$ magnetite peak. This shoulder peak is indicative of small amounts of maghemite $\left(\gamma-\mathrm{Fe}_{2} \mathrm{O}_{3}\right)$ [Jub10, Wil18], which was only observed for the web-like structure.

Overall, the Raman analysis shows that annealing $\mathrm{FeSe}_{1-x} \mathrm{Te}_{x}$ single crystals in air leads to the formation of magnetite and hematite on exposed surfaces of the sample. The layer beneath the blue surface layer of remains free of iron oxides. In some cases, a hematite-rich web-like structure formed on top of the blue surface layer during air annealing, which is likely to be related to the thickness of the sample, annealing conditions and the types of defects in the sample. Nonetheless, the web-structure could not reliably be reproduced and the exact origin still remains unclear at this point.

The oxidation of the inner surface of the cleaved samples shown in figure 4.6 and 4.7 might at first seem contradicting to the claim that the oxide layer only forms on the surface of the sample and not the bulk beneath. However, as explained above, the oxidation of inner surfaces is a result of the weakly-bound layered structure of $\mathrm{FeSe}_{1-x} \mathrm{Te}_{x}$, which promotes the formation of gap-defects through which oxygen diffuses into the sample. The formation of iron oxides was therefore also limited to the surfaces with direct contact to air or, more precisely, oxygen and not the bulk of the sample itself. Overall, the Raman analysis compliments the notion that the formation of iron oxides during annealing is limited to regions, where the surface 
of the sample is exposed and in direct contact with oxygen. This point is further reinforced by the XPS results presented later in section 4.6

\subsection{Cross-sectional Raman spectroscopy and SEM analysis}

In this section, the results from Raman spectroscopy and SEM analysis of the cross section of a $\mathrm{FeSe}_{0.35} \mathrm{Te}_{0.65}$ single crystal are analysed. This allows the investigation of not only the surface layers and underneath, but also regions further into the single crystals. Specifically, changes in the chemical composition of the subsurface and further into the bulk are analysed to determine the extend of the oxidation process and the thickness of the iron oxide layer. The thickness of the iron oxide layer is especially interesting, as it is directly proportional to the amount of iron that has been extracted from the bulk of the sample.

\subsubsection{Raman spectroscopy}

Figure 4.9 shows an optical microscope image of the cross section of an air-annealed $\mathrm{FeSe}_{0.35} \mathrm{Te}_{0.65}$ single crystal. The crystal was first embedded in epoxy, cured, and then mechanically polished to obtain a smooth surface for the cross-sectional Raman spectroscopy and SEM analysis. The embedding in epoxy is essential, as polishing would otherwise lead to exfoliation along the a-b plane due to the layered nature of this compound. This can still be seen in the area on the right side of the crystal marked by the red ellipse (see figure 4.9), where the crystal was already split in two before the potting process and the gap was consequently filled with the epoxy. To avoid confusion, it is worth noting that the 'lines' on the surface of the sample are not related to the orientation of the a-b plane or the general crystal orientation but are simply polishing scratches created during the mechanical polishing. The actual crystal orientation is indicated by the arrow pointing along the $\mathrm{c}$ axis of the crystal.

Figure 4.10(a) shows an enlargement of the microscope image of figure 4.9 for the area 


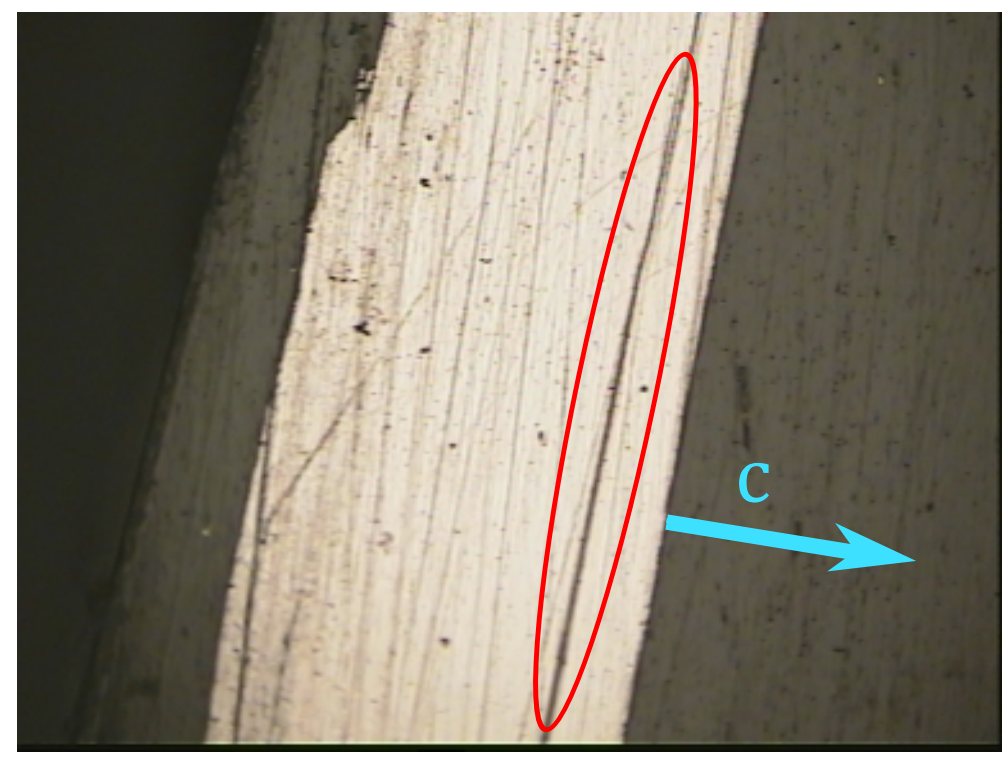

Figure 4.9: Optical microscope image of the cross section of an epoxy potted air-annealed $\mathrm{FeSe}_{0.35} \mathrm{Te}_{0.65}$ single crystal prepared for Raman spectroscopy and SEM analysis. The area marked by the red ellipse highlights a region, where the crystal was split. The light blue arrow indicates the c axis of the crystal structure.

of the cross section that was analysed by Raman spectroscopy. The star markers, which colours are linked to the colours of the spectra in figure 4.10(b), indicate the positions at which a spectrum was taken. For ease of comparison, the spectra have been normalised and shifted along the y-axis. The Raman spectrum at the bottom of figure 4.10(b) (dark blue) represents the first spectrum taken at the left side of the sample and the spectrum at the top (dark red) relates to the last spectrum taken at the right side of the sample. Selected, characteristic, and non-normalised spectra are later shown in figure 4.11 for a more detailed analysis.

Overall, Raman modes of magnetite and hematite are not uniformly detected throughout the cross section of the sample, but only in a few areas. Based on the analysis of the previous section, this is expected as iron oxide was shown to only form on the top and bottom surface of the sample or on surfaces exposed to oxygen by gaps in the layered structure. In addition and contrary to the previous analysis, some spectra also indicate the presence of small amounts of FeO, wuestite [DeF97]. Given that wuestite has never been observed in any other sample, the formation of 

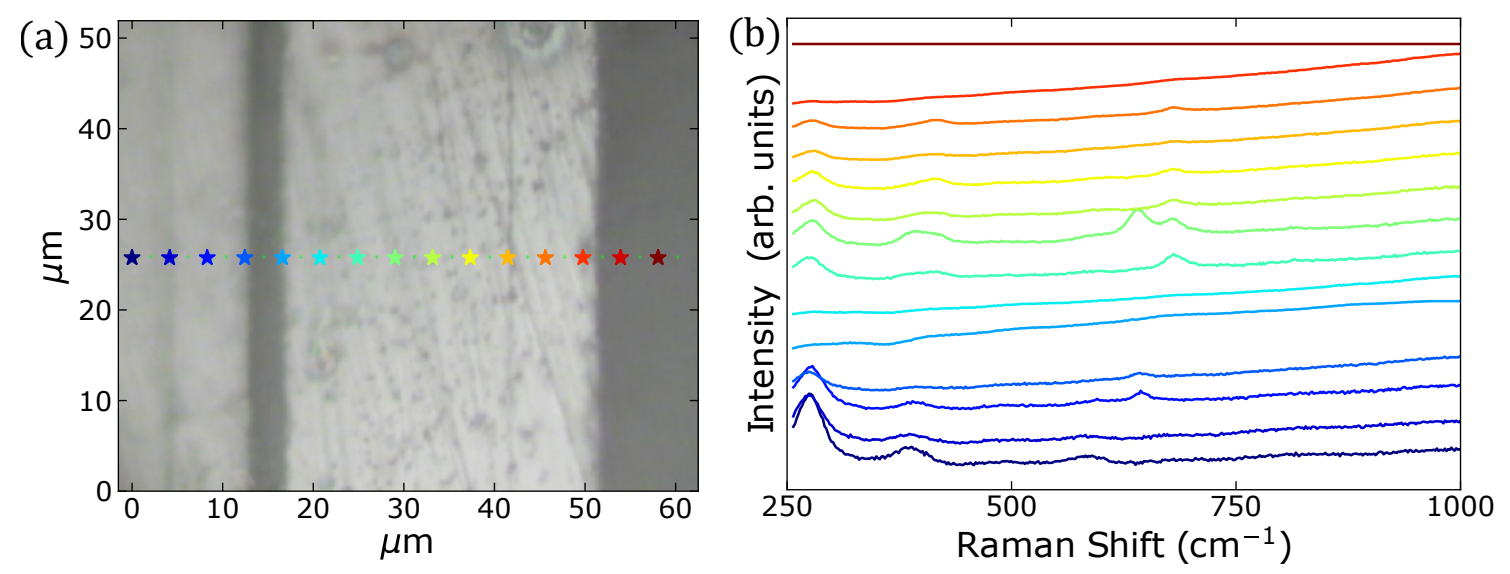

Figure 4.10: (a) Optical microscope image of the cross section of an air-annealed $\mathrm{FeSe}_{0.35} \mathrm{Te}_{0.65}$ single crystal. The differently coloured stars indicate the position at which a given Raman spectrum was taken. (b) Raman spectra taken at different positions of the crystal's cross section. The colour of the different spectra correlates to the colour of the star markers shown in (a).

it is surprising and likely to be a result of the cross section preparation. One possible explanation would be the oxidation of the freshly polished surface right after the polishing or during the Raman analysis. As has been shown by Faria et al., the laser of a Raman spectrometer itself can induce the formation of iron oxides or the transition from one oxidation state to another [DeF97]. Therefore, the combination of the freshly polished surface with the Raman spectroscopy might have led to the formation of, or transition to wuestite.

In this context, it should be noted that the peak size in figure 4.10 (b) might be misleading, as the intensity of each spectrum has been normalised to the maximum intensity of a given spectrum for ease of comparison and to fit in one overview graph. However, due to the fluorescent nature of the epoxy in which the sample is embedded, spectra taken close to epoxy have an increased background and thus exhibit a smaller peak height relative to the background. This is best illustrated by the dark red spectra in figure 4.10 (b) that is fully saturated and the spectrum beneath that barely shows any peaks due to large fluorescent background. As a result, some spectra with significant background appear to have smaller peaks and, on the contrary, spectra with low background, such as the first two blue spectra, 

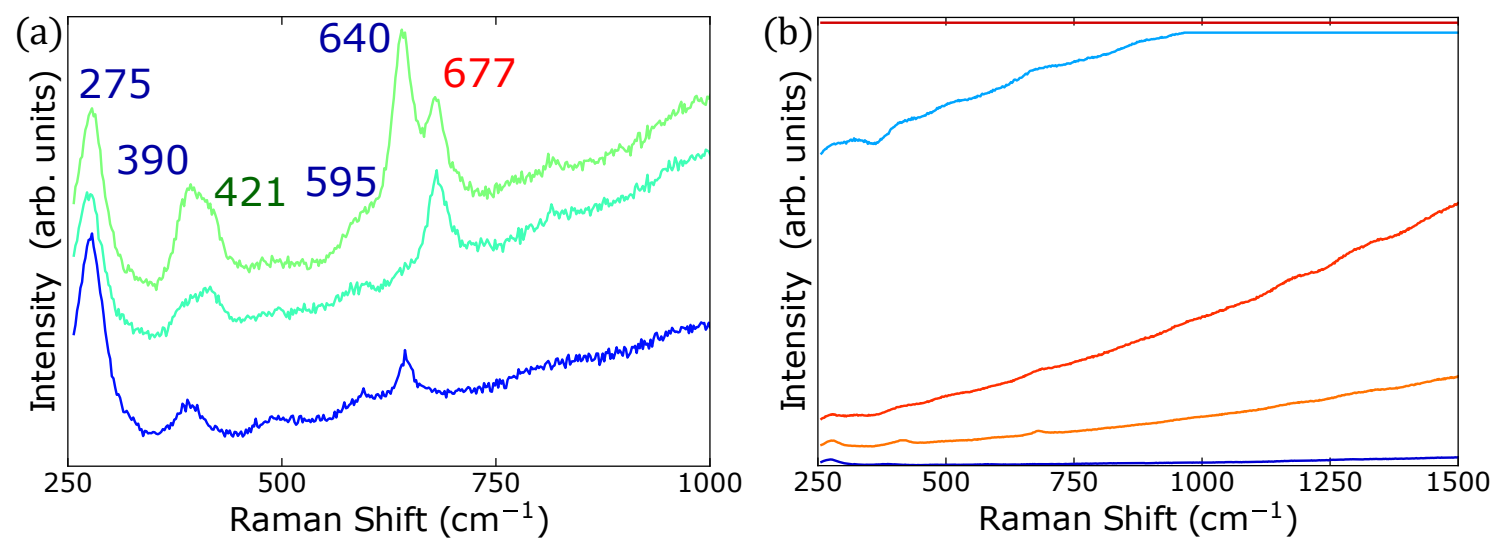

Figure 4.11: (a) Non-normalised Raman spectra for three characteristic areas of the cross section (see figure 4.10(a)). The Raman modes shown in the graph are magnetite $\left(677 \mathrm{~cm}^{-1}\right)[]$, hematite $\left(421 \mathrm{~cm}^{-1}\right)$, and wuestite $\left(275 \mathrm{~cm}^{-1}, 390 \mathrm{~cm}^{-1}, 595 \mathrm{~cm}^{-1}\right.$, and $\left.640 \mathrm{~cm}^{-1}\right)$ [DeF97]. (b) Non-normalised Raman spectra of areas with significant fluorescent background and low background (dark blue).

appear to have large peaks. This is however not related to the amount of iron oxide in that area, but rather due to the normalisation and the fluorescent background.

Figure 4.11 shows two selections of characteristic spectra from the overview shown in figure $4.10(\mathrm{~b})$. The colour of the spectra is again representative of the position of the measurement as marked in figure 4.10(a), however, the spectra here have not been normalised. The spectra shown in figure 4.11(a) exhibit two characteristic sets of Raman peaks that are repeatedly found in the cross-sectional spectra. The blue spectrum exhibits peaks at $275 \mathrm{~cm}^{-1}, 390 \mathrm{~cm}^{-1}, 595 \mathrm{~cm}^{-1}$, and $640 \mathrm{~cm}^{-1}$. These peaks are, to some extent, seen in most spectra and are the Raman modes of wuestite [DeF97]. The cyan spectrum above also shows these peaks, however, some peaks are almost indistinguishable from the background. Additionally, the cyan spectrum also exhibits a pronounced peak at $421 \mathrm{~cm}^{-1}$ and $677 \mathrm{~cm}^{-1}$, which are Raman modes of hematite and magnetite, respectively. The mixture of the different oxides is best illustrated in the light green spectrum. The two Raman modes at $640 \mathrm{~cm}^{-1}$ and $677 \mathrm{~cm}^{-1}$ of wuestite and magnetite are both pronounced. Furthermore, the peak around $400 \mathrm{~cm}^{-1}$ is broadened as a result of the mixing of the wuestite and the hematite peak $\left(390 \mathrm{~cm}^{-1}\right.$ and $\left.421 \mathrm{~cm}^{-1}\right)$. 
Figure 4.11(b) illustrates the significant background due to the fluorescent epoxy as showcased by the orange to red spectra on the right side of the sample and the spectra taken at the sample gap at $15 \mu \mathrm{m}$ (see figure 4.10(a)). Starting with the orange to dark red spectra, the background continuously increases when moving closer to the epoxy and, in fact, completely saturated the detector in case of the last two spectra taken on the epoxy (see figure 4.10(a)). Similarly, the light blue spectrum taken at the epoxy-filled gap in the middle of the cross section $(15 \mu \mathrm{m})$, is also partially saturated. The magnitude of this background is best illustrated by comparison to the dark blue, low-background spectrum taken in the bulk region of the sample. Unfortunately, this background due to fluorescence prevents a detailed analysis of areas close to the epoxy, such as the surface iron oxide layer that is expected at the interface between the sample and the epoxy, and thus warrants the investigation by other means.

\subsubsection{SEM analysis}

To complement the cross-sectional Raman analysis above, SEM imaging was carried out on the same $\mathrm{FeSe}_{0.35} \mathrm{Te}_{0.65}$ crystal cross section. Figure 4.12 shows a backscatter detector SEM image of the sample cross section at the surface interface. As introduced in chapter 3.6. the brightness of an area of the image is determined by the atomic number of the element(s) in that area: the brighter it is, the larger the atomic number [Llo87].

The image in figure 4.12 exhibits three characteristic features: a rough dark surface layer with a thickness averaging around $100 \mathrm{~nm}$, a bright layer of slightly larger thickness underneath, and the remaining bulk of the sample. Based on the Z-contrast of the backscatter image, the dark layer must consist of elements with the lowest atomic number compared to the rest of the sample. Therefore, this layer is most likely to be the surface iron oxide layer with Fe (26) and $\mathrm{O}(8)$ having lower atomic numbers compared to Se (34) and Te (52). The bright layer beneath this oxide layer 


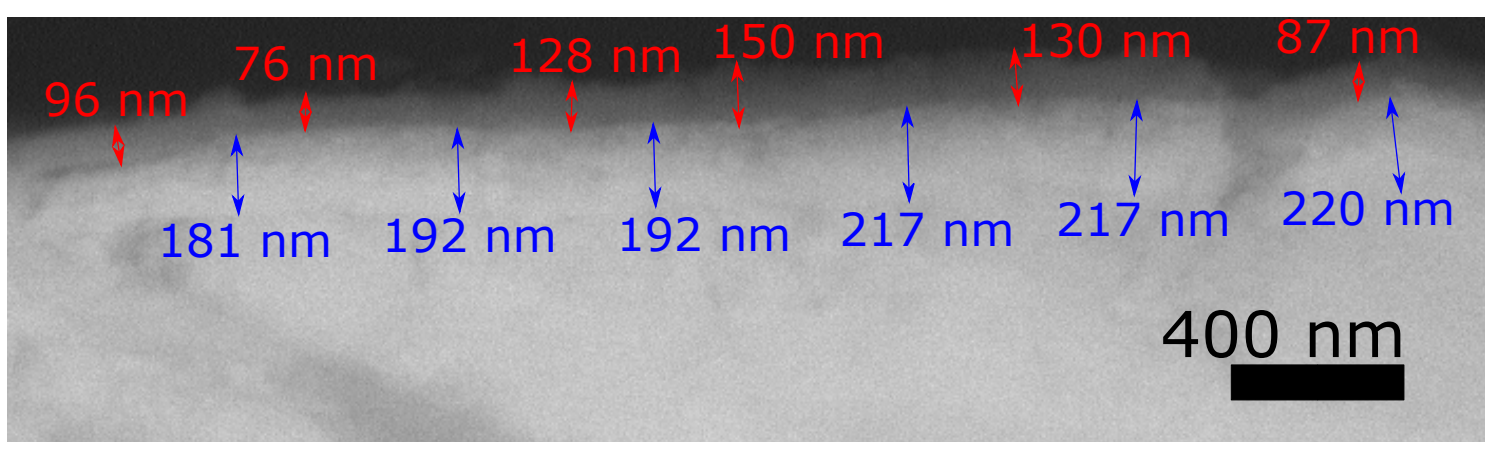

Figure 4.12: Backscatter SEM image of the cross section of an air-annealed $\mathrm{FeSe}_{0.35} \mathrm{Te}_{0.65}$ sample. The brightness of an area of the image is related to the atomic number of the elements in that area: the brighter it is, the larger the atomic number [Llo87].

is surprising and has not been reported before. Given the sample consist of Fe, Se, and Te, with Fe being the element with the lowest atomic number of the three, the increased brightness of this layer indicates an increased Se and Te concentration and a lower Fe concentration compared to the bulk beneath this 'depletion layer'. Further into the sample, the brightness of the bulk is again reduced, indicating an increase in the Fe content relative to the Se and Te concentrations.

These results align well with the formation of a thin iron oxide layer on the surface of $\mathrm{FeSe}_{1-x} \mathrm{Te}_{x}$ single crystals. Iron is drawn out of the sample and reacts with the oxygen at the surface of the sample during annealing. This leads to a reduction in the amount of iron within the sample and therefore a relative increase of the selenium and tellurium concentration. Interestingly, as indicated by the bright layer, this process is limited in depth. The iron is only drawn out from the region right underneath the iron oxide and not from the whole sample. Intuitively, this is reasonable as iron is not expected to be able to diffuse from deep inside to the surface of the sample at these temperatures [Lau14].

\subsection{Cross-sectional STEM analysis}

Extending on the investigation of the previous section, the cross section of a different air-annealed $\mathrm{FeSe}_{0.35} \mathrm{Te}_{0.65}$ sample was analysed using scanning transmission electron 


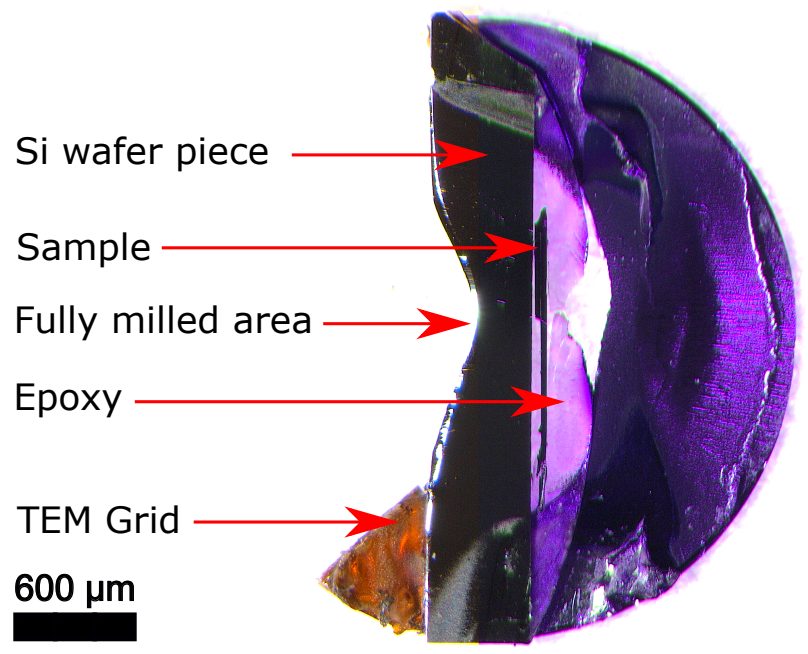

Figure 4.13: Optical microscope image of the epoxy embedded $\mathrm{FeSe}_{0.35} \mathrm{Te}_{0.65}$ single crystal. The sample was fixed on a TEM grid together with a piece of silicon wafer for stability and then ion milled to obtain a sample thickness suited for STEM.

microscopy (STEM). This allows for higher resolution analysis of the cross section and furthermore acts as a consistency check between different samples and sample preparation processes. Similar to the previous sample, this sample was first glued on a silicon wafer piece for stability, embedded in epoxy, and mechanically polished to a thin slide. However, the sample has not been analysed by Raman spectroscopy to avoid changes in the surface chemistry due to the Raman laser and instead has been fixed to a TEM grid and milled to a thickness suitably for STEM in an added last step using an argon ion mill.

The whole slide assembly on the TEM grid after argon ion milling is shown in figure 4.13. The two dips, one on the epoxy and sample side and the other on the Si wafer side, indicate the areas that have been fully milled by the argon ion beam. Further into the sample and the wafer, an extremely thin cross section of the sample suitable for STEM remains.

Figure 4.14 shows a high-magnification, annular dark-field STEM image of the surface region of the air-annealed crystal. Similar to the SEM analysis of the other sample presented in the previous section, the surface of this sample also exhibits a 


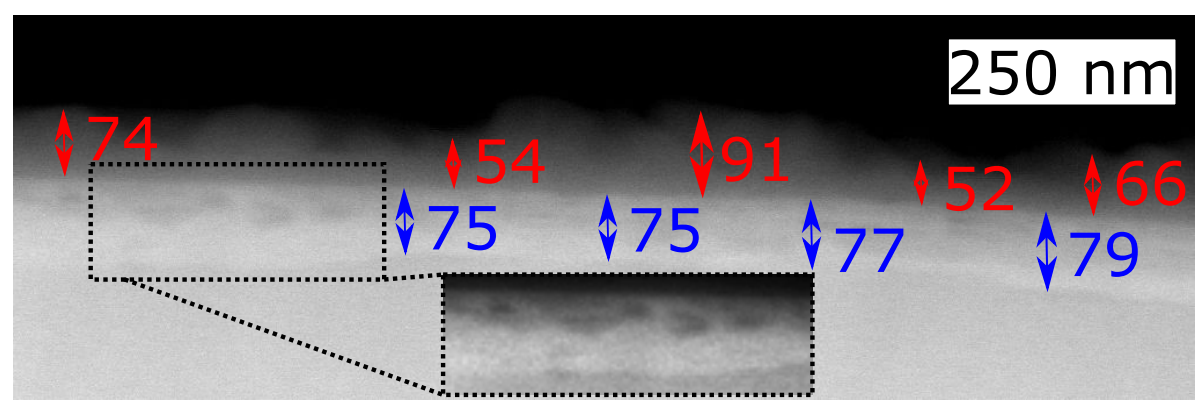

Figure 4.14: High-magnification, annular dark-field STEM image of the cross section of an air-annealed $\mathrm{FeSe}_{0.35} \mathrm{Te}_{0.65}$ sample. The brightness of an area of the image is related to the atomic number of the elements in that area: the brighter it is, the larger the atomic number [Nel08].

rough dark layer on the topmost surface and a brighter layer of similar thickness just underneath. Based on the Z-contrast [Nel08] and analogous to the SEM analysis, the dark layer is likely to consist of iron oxides. The bright layer underneath is slightly brighter than the bulk of the sample, which insinuates an iron deficient $\mathrm{Fe}_{y-\delta} \mathrm{Se}_{0.35} \mathrm{Te}_{0.65}$ layer. Overall, both the iron deficient and the iron oxide layer of this sample are thinner than that of the SEM sample but still on the same order of magnitude. This might be the result of slightly varying surface conditions of the sample and the weak contrast in the backscatter SEM image compared to the STEM image.

On another note, both the STEM and SEM analysis show a large variance in the oxide layer thickness compared to the iron-deficient layer with almost constant thickness. This insinuates preferential oxidation of some parts of the surface compared to others. One possible explanation for this could be the existence of defects through which the interstitial excess iron can diffuse more easily in the out-of-plane direction and through the layered structure [Lau14]. The resulting iron concentration gradients in the ab-planes are then quickly balanced out due to the fast in-plane diffusion [Lau14]. Because of this, the removal of excess iron and the thickness of the iron-depleted layer is relatively homogenous even with a varying iron oxide formation. This notion is elaborated in more detail in the proposed diffusion model presented in section 4.8 . On a closer look, the grey-scale and contrast-adjusted enlargement of the bright layer 


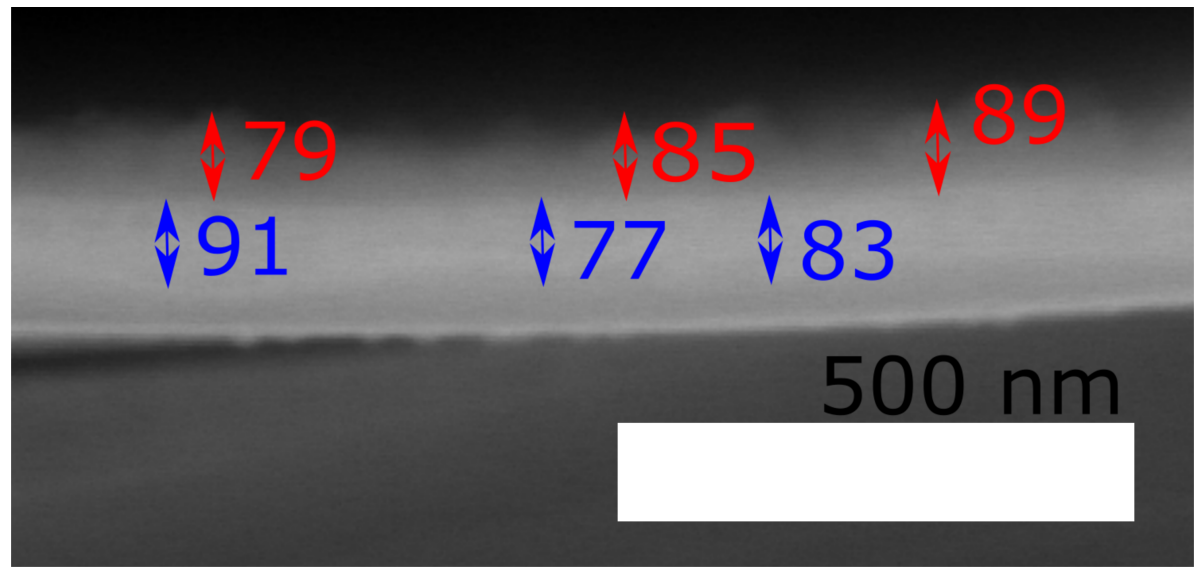

Figure 4.15: High-magnification, annular dark-field STEM image of an exfoliated surface layer of the air-annealed $\mathrm{FeSe}_{0.35} \mathrm{Te}_{0.65}$ sample. The brightness of an area of the image is related to the atomic number of the elements in that area: the brighter it is, the larger the atomic number Nel08.

(see inset of figure 4.14) shows 'dark pockets' in the upper region of the bright layer. Based on the brightness, these pockets are likely to consist of iron oxide. Similar to the argument of the previous section, this is likely to be caused by small, localised defects in the crystal structure. These defects enable oxygen to diffuse into the upper sub-surface region and react with the iron to form pockets of iron oxide. However, there is no evidence of oxide pockets in the lower regions of the bright layer and beneath.

Figure 4.15 shows a high-magnification STEM image of an exfoliated surface layer. Due to the very low thickness of the sample and the two dimensional nature of $\mathrm{FeSe}_{0.35} \mathrm{Te}_{0.65}$, the heating effect of the electron beam of the electron microscope alone was able to exfoliate a surface layer. This again illustrates, that even the characterisation of the surface structure itself can alter the surface. Still, the exfoliated layer shows a rough dark layer on the top and a bright layer underneath, similar to that observed for the other surface of the same sample in figure 4.14 and the SEM analysis of the previous sample in figure 4.12 . 

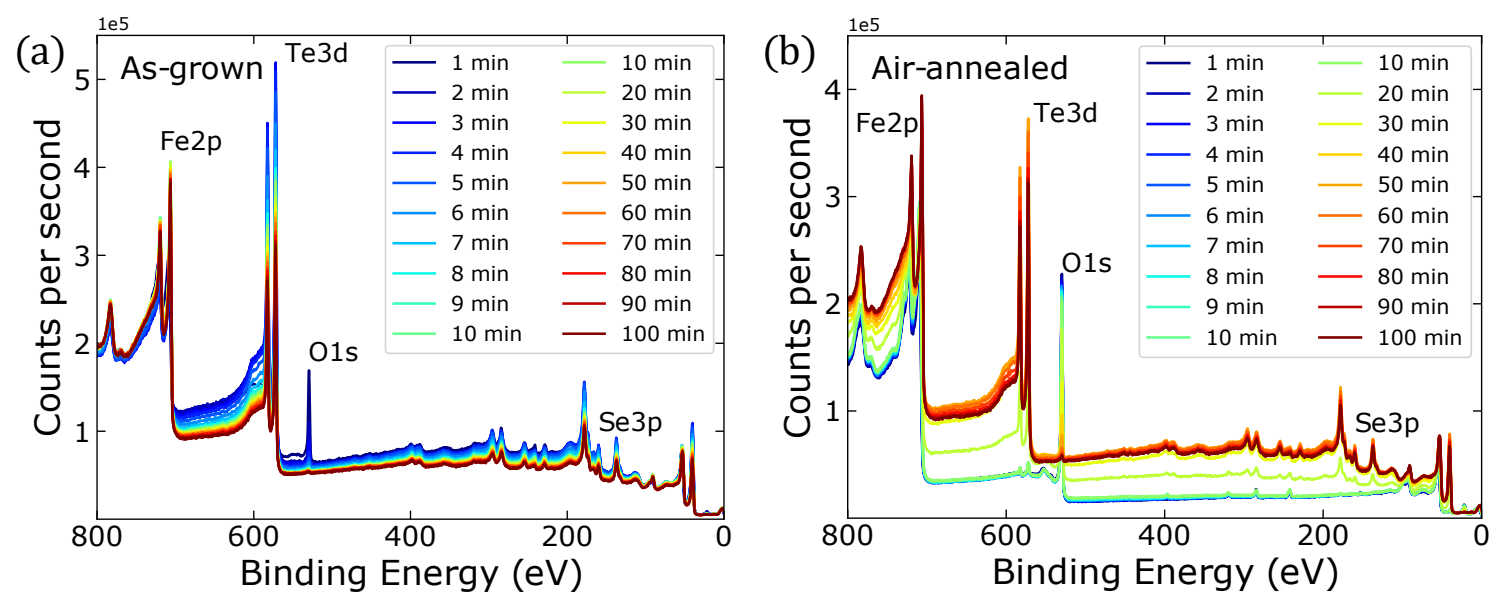

Figure 4.16: XPS spectra of the as-grown (a) and the air-annealed (b) $\mathrm{FeSe}_{0.35} \mathrm{Te}_{0.65}$ single crystal. The colour is representative of the ion-beam milling time that is proportional to the analysed depth of the sample. Characteristic XPS peaks of $\mathrm{Fe}, \mathrm{Te}, \mathrm{Se}$, and $\mathrm{O}$ are marked in both graphs.

\subsection{Depth-profiling X-Ray photoelectron spectroscopy (XPS) analysis}

The backscatter SEM and STEM analysis presented in the previous sections indicates an iron oxide thickness around $100 \mathrm{~nm}$ and the presence of an iron-deficient layer just beneath the iron oxide. However, the analysis is solely based on the Zcontrast of backscatter SEM and annular dark-field STEM and thus warrants further complementary analysis to confirm the composition of these different layers.

In this section, the results from depth-profiling X-Ray photon spectroscopy (XPS) analysis of one as-grown and one air-annealed $\mathrm{FeSe}_{0.35} \mathrm{Te}_{0.65}$ single crystal are presented. Using an in-situ argon ion beam, the samples were milled stepwise and XPS spectra were taken after every milling step to obtain a composition depth-profile of the two samples.

Figure 4.16 shows two sets of XPS survey scans of the as-grown (a) and the airannealed (b) $\mathrm{FeSe}_{0.35} \mathrm{Te}_{0.65}$ single crystal over the whole milling range. Both samples were ion milled following the same time steps, milling pressure, energy, and current to obtain a comparable depth profile. Although quantitative conclusions cannot be 

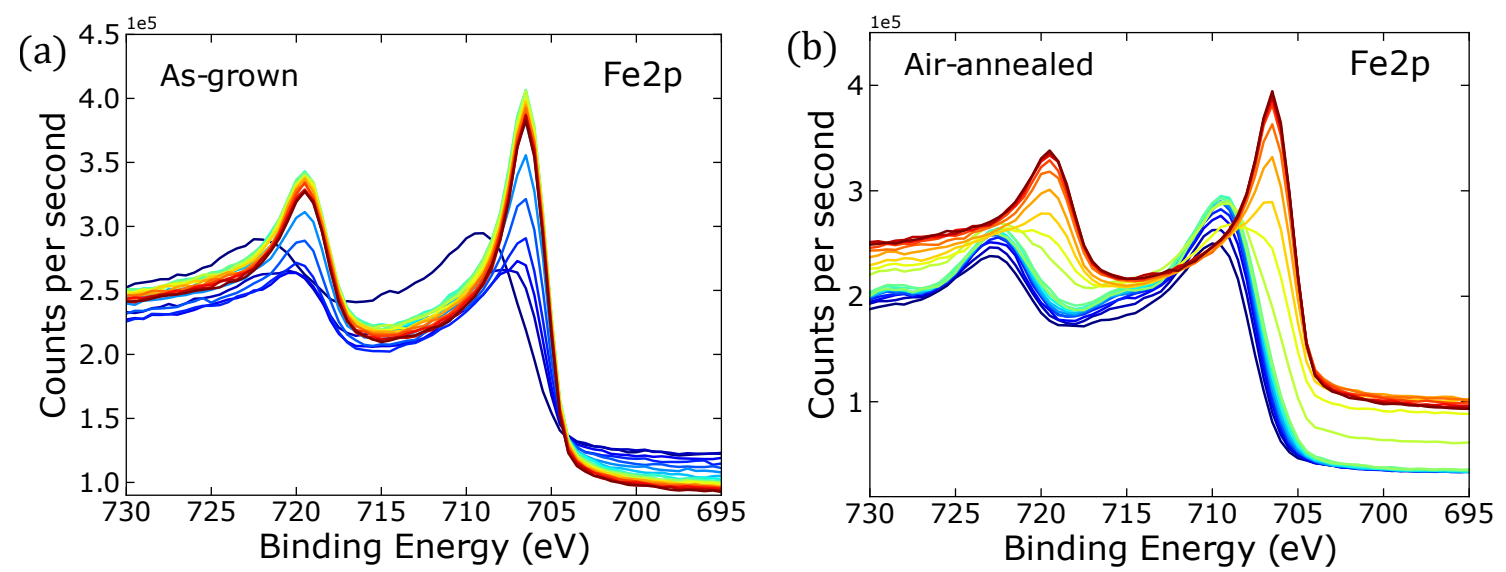

Figure 4.17: Enlargement of the Fe2p XPS peaks of an as-grown (a) and an air-annealed (b) $\mathrm{FeSe}_{0.35} \mathrm{Te}_{0.65}$ single crystal. The colour is representative of the ion-beam milling time as indicated in figure 4.16 .

extracted from the whole set of survey spectra stacked on top of each other over the whole range, some qualitative differences between the as-grown and the air-annealed sample are revealed. Overall, the spectra of the as-grown sample display little change for all milling times. On the other hand, the spectra of the air-annealed sample change significantly from low milling times (blue colours) to high milling times (red colours). This is best illustrated by the oxygen peak that is more pronounced in the spectra of the air-annealed sample in comparison to the as-grown sample, where the O1s peak vanishes after a few minutes of milling. In general, this behaviour is expected, as the annealing process has already been shown to change the composition of the near-surface region in the earlier sections of this chapter. For a more detailed analysis on how the concentration of each element evolves with depth, an enlargement of the four characteristic XPS peaks ( $\mathrm{Fe} 2 \mathrm{p}, \mathrm{Te} 3 \mathrm{~d}, \mathrm{O} 1 \mathrm{~s}$ and $\mathrm{Se} 3 \mathrm{p}$ ) is plotted in figure 4.17 to figure 4.20

Figure 4.17 shows an enlargement of the XPS Fe2p region for the as-grown (a) and air-annealed (b) sample. Again, the colour of the different spectra is representative of the milling time from 1 minute of milling in dark blue to dark red for a total of 100 minutes of milling. Starting with the as-grown sample, the spectrum after 1 minute of milling (dark blue) exhibits broad peaks centred at $709.2 \mathrm{eV}$ and $722.2 \mathrm{eV}$. These 
broad peaks are indicative of a combined composition of several oxidation states of iron that lead to a superposition of several slightly shifted peaks. Based on the Raman analysis above, the surface (1 minute of milling) is likely to consist of both hematite and magnetite. The respective Fe ion binding energies are $709.7 \mathrm{eV}$ and $722.7 \mathrm{eV}$ for $\mathrm{Fe}^{2+}$ ions and $711.1 \mathrm{eV}$ and $724.2 \mathrm{eV}$ for $\mathrm{Fe}^{3+}$ ions [Gra96, Gra98, Fuj99, which matches the observed peak position. With increasing milling time of up to 7 minutes of milling, the two peaks continuously become sharper and shift towards lower binding energies centred at $706 \mathrm{eV}$ and $720 \mathrm{eV}$. These peak positions are similar to that of $\mathrm{FeSe}_{1-x} \mathrm{Te}_{x}$ [Tel12], which are found at $706.8 \mathrm{eV}$ and $719.8 \mathrm{eV}$ for $\mathrm{Fe} 2 \mathrm{p}_{3 / 2}$ and $\mathrm{Fe} 2 \mathrm{p}_{1 / 2}$, respectively. This result indicates that the surface oxide is being milled off and the Fe2p binding energies shift from the oxide state to the more metallic $\mathrm{FeSe}_{1-x} \mathrm{Te}_{x}$ state. Deeper into the sample, that is for milling times over 7 minutes, the peak position and width remain unchanged.

For the air-annealed sample, a qualitatively similar behaviour is observed. However, it is not only the first spectrum that shows broadened Fe $2 \mathrm{p}_{3 / 2}$ and $\mathrm{Fe} 2 \mathrm{p}_{1 / 2}$ peaks, but these broadened peaks were observed even after up to 10 min of milling. Moreover, the spectra taken after several minutes of milling exhibit characteristic shake-up satellite peaks of $\mathrm{Fe}^{2+}$ at about $715 \mathrm{eV}$ and $728 \mathrm{eV}$ Gra96, Gra98, Fuj99. These satellites are due to Fe ions left in an excited state, which lowers the kinetic energy of the detected photoelectron by a couple $\mathrm{eV}$ and thus leads to a higher estimate of the binding energy for the satellite peaks [Mou92]. The first couple spectra, however, do not exhibit these satellite peaks. This indicates that the iron oxide composition changes with depth. At first, the satellites are washed out by the superposition of the $\mathrm{Fe}^{2+}$ and $\mathrm{Fe}^{3+}$ satellites [Gra96, Gra98, Fuj99, but a relative reduction in $\mathrm{Fe}^{3+}$ ions after several minutes of milling exposes the satellite peaks of $\mathrm{Fe}^{2+}$.

At longer cumulative milling times, the two Fe2p peaks become increasingly sharper and shift towards lower binding energies, centred at $706 \mathrm{eV}$ and $720 \mathrm{eV}$. However, the transition to sharper peaks for the air-annealed sample is stretched out over more than 70 minutes of milling time compared to just 7 minutes for the as-grown 

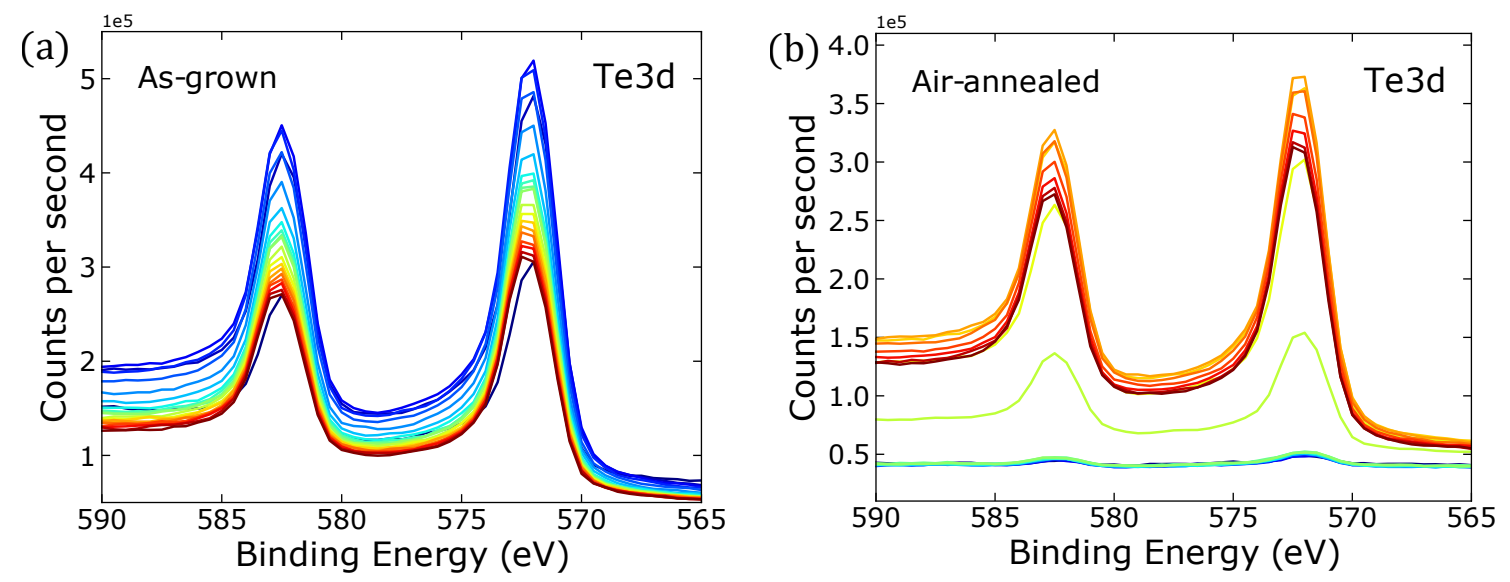

Figure 4.18: Enlargement of the XPS Te3d spectra of an as-grown (a) and an air-annealed (b) $\mathrm{FeSe}_{0.35} \mathrm{Te}_{0.65}$ single crystal. The colour is representative of the ion-beam milling time as indicated in figure 4.16 .

sample. For milling times over 70 minutes, the XPS peak position and width remain unchanged.

In general, the argon ion beam milling rate depends on several sample-specific factors such as the sample composition, surface topography, and density. Unfortunately, because of the limited sample size and measurement equipment availability, the milling rate could not specifically be determined for the as-grown and air-annealed samples presented here. However, using similar milling parameters, the milling rate for $\mathrm{Ta}_{2} \mathrm{O}_{5}$ has previously been calibrated to be on the order of $1 \mathrm{~nm}$ per minute. Assuming a similar milling rate of $1 \mathrm{~nm}$ per minute for the $\mathrm{FeSe}_{0.35} \mathrm{Te}_{0.65}$ sample, the thickness of the iron oxide layer of the annealed sample would be on the order of $70 \mathrm{~nm}$. Given the crude approximation, this estimate matches very well with the STEM results of the surface layer reported in the previous section. For the as-grown sample, the iron oxide layer would therefore just be a couple nanometre thick. This is also consistent with the XPS detection depth. Since both tellurium and selenium are detected in the first spectrum, the iron oxide thickness must be smaller than the XPS detection depth, which is also estimated to be on the order of several nanometres.

The enlargement of the XPS Te3d peaks for the as-grown (a) and air-annealed (b) sample are shown in figure 4.18. Similar to the Fe2p peaks, the Te3d peaks are 
also split in two peaks due to spin orbit splitting with the $\mathrm{Te}_{3} \mathrm{~d}_{5 / 2}$ and $\mathrm{Te}_{3} 3 \mathrm{~d}_{3 / 2}$ peak at $573 \mathrm{eV}$ and $583 \mathrm{eV}$, respectively [Tel12]. However, there are no satellite peaks associated with the Te3d level and the peak positions remain unchanged for all spectra. This suggests that the tellurium does not form multiple phases with different oxidation states. Specifically, there is no evidence for the formation of tellurium oxide, which peaks are expected around $576 \mathrm{eV}$ [Gar81].

For the as-grown sample, the peak height intensity of the XPS Te3d peaks is the lowest for the first spectrum, but increases rapidly with milling time reaching a maximum peak height after two minutes of milling. This correlates well with the evolution of the Fe2p peaks above. The first couple nanometres of the sample consist predominantly of iron oxide and therefore the tellurium signal is very low. After a couple minutes of milling, the oxide is removed and the tellurium peak quickly rises. It is worth noting though, that the tellurium peak is again reduced after three minutes of milling. This is surprising, as it insinuates that the tellurium content is higher close to surface and reduced going deeper into the sample, seemingly approaching a stable level.

The Te3d XPS peaks of the air-annealed sample show a qualitatively similar behaviour, however, the changes happen deeper in the sample. The spectra taken between $1 \mathrm{~min}$ and $20 \mathrm{~min}$ of milling only show minute Te peak intensities. This matches the result of the Fe2p peaks that showed that the surface of the sample consists predominately of iron oxide and revealed a transition from the oxide covered surface to an oxide-free surface after 20 minutes of milling time. Additionally, the peak height intensity reaches a maximum value just after the iron oxide has been milled off after 40 minutes of milling. After that, the peak height intensity again decreases slightly before leveling out, similar to what was observed in the as-grown sample.

Figure 4.19 shows the O1s XPS peak at $529.5 \mathrm{eV}$ for the as-grown (a) and air-annealed sample (b). The peak height intensity of the O1s peak decreases rapidly for the asgrown sample and completely disappears after 7 minutes of milling. This is directly 

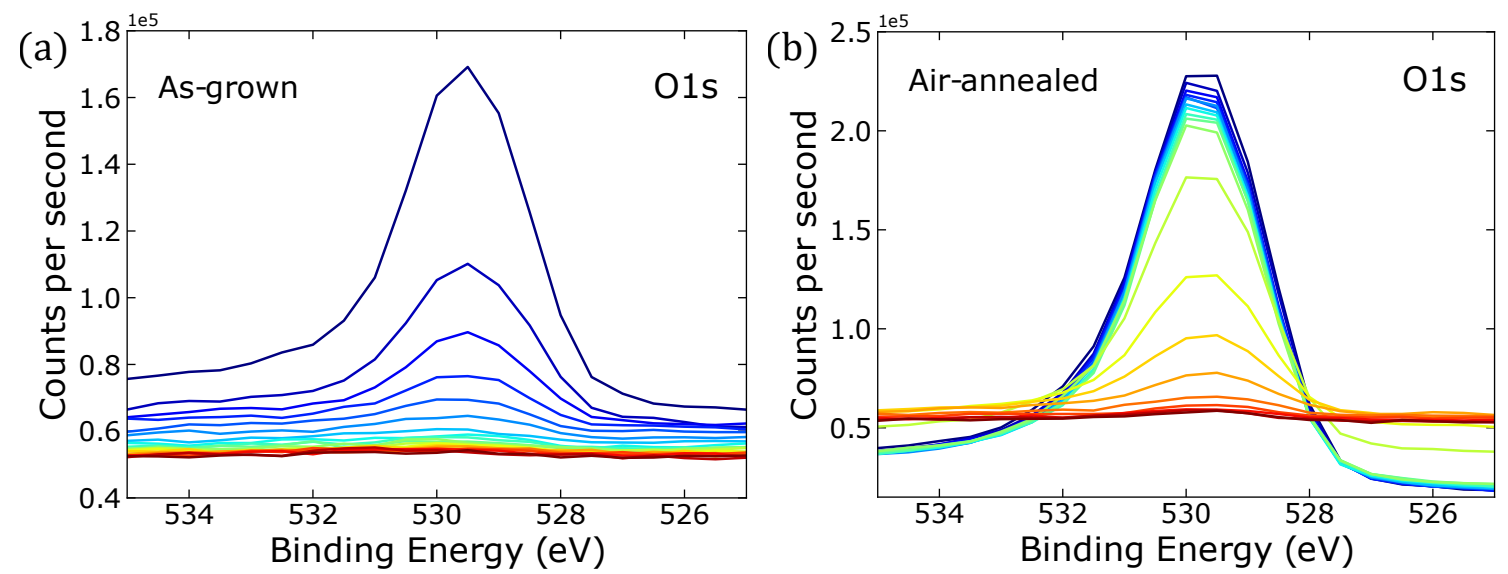

Figure 4.19: Enlargement of the XPS O1s spectra of an as-grown (a) and an air-annealed (b) $\mathrm{FeSe}_{0.35} \mathrm{Te}_{0.65}$ single crystal. The colour is representative of the ion-beam milling time as indicated in figure 4.16

related to the presence of iron oxides on the surface due to the exposure to air during transportation and the presence of oxygen in the storage desiccator. It will later be shown in the chapter 6 that even a $\mathrm{N}_{2}$ (oxygen $<5 \mathrm{ppm}$ ) or, analogously, Ar atmosphere contains enough residual oxygen to oxidise the surface of $\mathrm{FeSe}_{1-x} \mathrm{Te}_{x}$.

In contrast, the air-annealed sample was heat treated at $300^{\circ} \mathrm{C}$, which accelerated the oxidation process and promoted the diffusion of oxygen deeper into the sample. The oxygen content is still the highest in the near-surface region, but the O1s peak remains visible even after 70 minutes of milling, which relates to around $70 \mathrm{~nm}$. On a side note, one notices that the spectra for milling times above 30 minutes (orange to red colour) show an elevated background. This is due to the increased Se and Te content at this depth that leads to an increased background at higher binding energies. Some of the Se and Te photoelectrons undergo inelastic scattering, which leads to an increased level of background at binding energies higher than the peak energy Mou92].

Unfortunately, the most prominent selenium XPS peak, Se3d, overlaps with the Fe3p peak making it hard to separate the two contributions. Therefore, the second most prominent peak, Se3p, was chosen for the analysis. Still, because of the relatively low selenium concentration in this sample in comparison to the iron and tellurium 

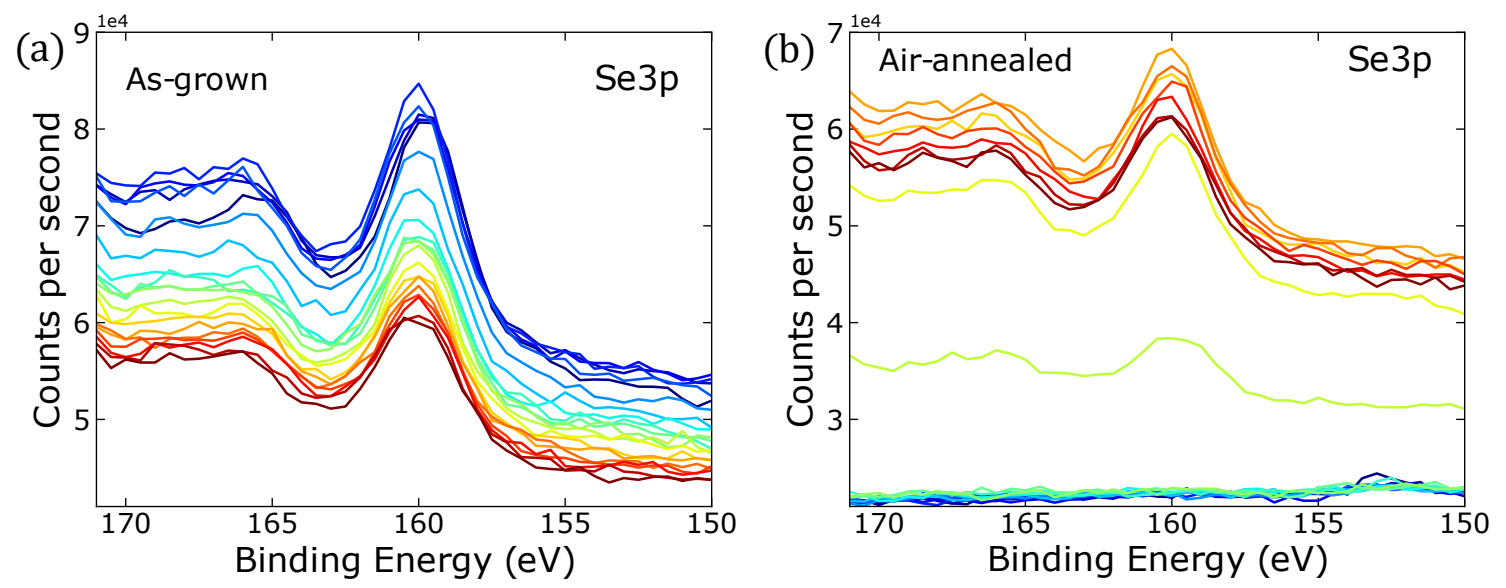

Figure 4.20: Enlargement of the XPS Se3p spectra of an as-grown (a) and an air-annealed (b) $\mathrm{FeSe}_{0.35} \mathrm{Te}_{0.65}$ single crystal. The colour is representative of the ion-beam milling time as indicated in figure 4.16 .

concentration (nominal concentration $\mathrm{FeSe}_{0.35} \mathrm{Te}_{0.65}$ ), the high RSF factor, and consequently low photoemission cross section, the Se3p peak only exhibits a very small signal as can be seen in the enlarged spectra shown in figure 4.20 for the as-grown (a) and air-annealed (b) sample. In fact, the Se $3 \mathrm{p}_{1 / 2}$ peak can hardly be distinguished from the background.

In spite of this, a quick qualitative analysis still shows that the behaviour is practically identical to the behaviour observed for Te. The as-grown sample first shows a slight increase for the first minutes of milling when the iron oxide surface layer was removed. After that, the peak height decreases slightly and then effectively stays constant. The spectra of the air-annealed sample show no Se peaks for the first 10 minutes of milling but then increase to a constant value after 50 to 60 minutes of milling. This is again in good agreement with the behaviour of the Te3d peak height with milling time.

To quantify the qualitative behaviour described above, the peak areas of the Fe2p, Te3d, O1s, and Se3p levels were fitted using CasaXPS2. First, the background of each peak region was subtracted using a Shirley function and the peaks were fitted using a sum of pseudo-Voigt functions. The fitting region boundaries and constraints

\footnotetext{
${ }^{2}$ CasaXPS, Casa Software Ltd., 5 Grosvenor Terrace, Teignmouth,TQ14 8NE, United Kingdom
} 


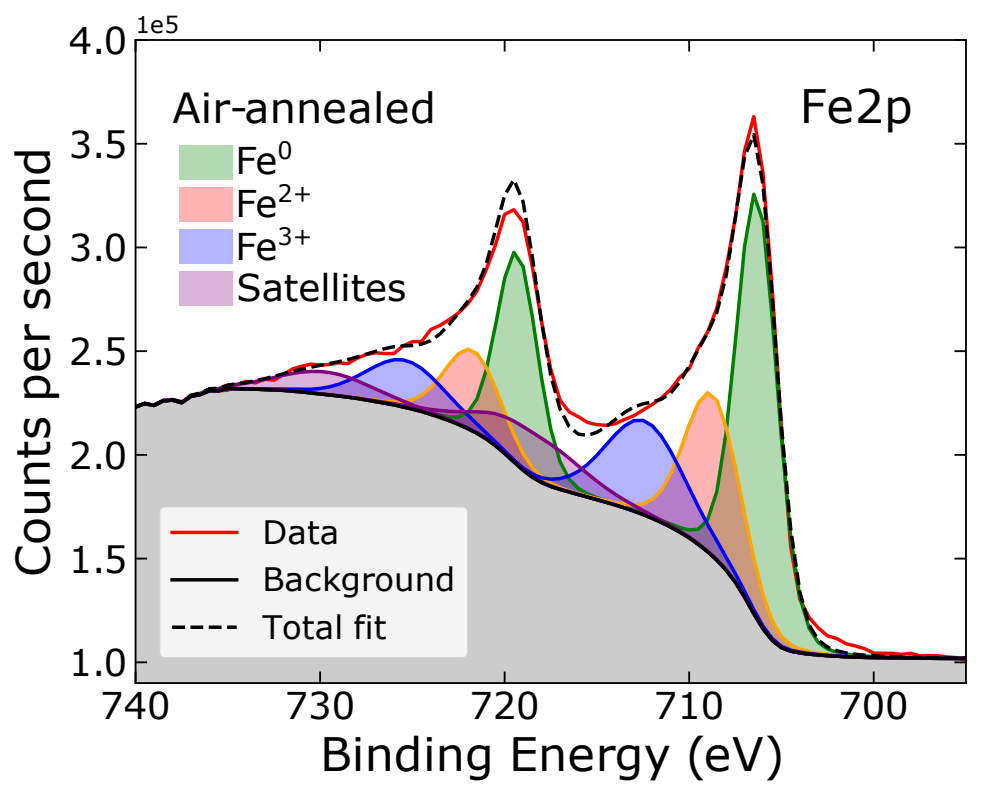

Figure 4.21: Enlargement of the Fe2p peaks of the XPS spectra of the air-annealed $\mathrm{FeSe}_{0.35} \mathrm{Te}_{0.65}$ single crystal after 30 minutes of milling. The two peaks were fitted using a Shirley background and 8 different pseudo-Voight functions.

of the XPS peaks of the different elements were chosen to be identical for all spectra taken at different milling time and for both the as-grown and air-annealed sample. More specifically, the starting value of a peak binding energy was set to the literature value for the specific species [Gra96, Gra98, Fuj99, Tel12]. The total area of each peak was then divided by the RSF, and normalised to the total RSF-corrected area of all elements. For ease of comparison, the concentration of each element has been normalised to their maximum value to better illustrate the qualitative changes in concentration between the different elements on the same scale.

Figure 4.21 shows an exemplary fit of the Fe2p peaks that were fit using 8 pseudoVoigt functions. The three different $\mathrm{Fe}$ oxidation states $\left(\mathrm{Fe}^{0}, \mathrm{Fe}^{2+}\right.$, and $\left.\mathrm{Fe}^{3+}\right)$ each contribute each least two peaks Gra96, Gra98, Fuj99. In the case of $\mathrm{Fe}^{2+}$ and $\mathrm{Fe}^{3+}$, there are additional satellite peaks and, technically, the same oxidation state can also exhibit slight variations in the peak position depending on the structure, e.g. tetrahedral $\mathrm{Fe}^{3+}$ vs octahedral $\mathrm{Fe}^{3+}$ in magnetite Fuj99. This leads to at least 10 deconvoluted peaks fitted onto the two observed peaks in the measured spectrum. In 
addition, the Fe species present also changes at different layers of the sample adding further complication to the fitting. Due to this complexity, fitting of the Fe peaks with the different oxidation states is extremely difficult. The fit results including an estimate of the concentration of the different oxidation states for this example are shown in table 4.3. However, fitting many peaks on two observable peaks adds a level of arbitrariness that limits the reliability of the relative concentrations. For this reason, the relative concentration of the single oxidation states is not analyzed in further detail, but the whole area, which is proportional to the total concentration, is considered.

Table 4.3: Summary of the Fe2p core level binding energies of $\mathrm{Fe}^{0}, \mathrm{Fe}^{2+}, \mathrm{Fe}^{3+}$ and the relative concentrations derived from the peak fits shown in figure 4.21. However, the relative concentrations can only be considered rough estimates due to the wide range of peak-fit combinations that are supported by the data.

\begin{tabular}{r|c|c|c} 
XPS core-level & oxidation state & Binding energy & relative concentration (\%) \\
\hline $\mathrm{Fe} 2 \mathrm{p}_{3 / 2}$ & $\mathrm{Fe}^{0}$ & $706 \mathrm{eV}$ & 0.51 \\
\hline $\mathrm{Fe} 2 \mathrm{p}_{1 / 2}$ & $\mathrm{Fe}^{0}$ & $719 \mathrm{eV}$ & 0.49 \\
\hline $\mathrm{Fe} 2 \mathrm{p}_{3 / 2}$ & $\mathrm{Fe}^{2+}$ & $708 \mathrm{eV}$ & 0.27 \\
\hline $\mathrm{Fe} 2 \mathrm{p}_{1 / 2}$ & $\mathrm{Fe}^{2+}$ & $722 \mathrm{eV}$ & 0.27 \\
\hline $\mathrm{Fe} 2 \mathrm{p}_{3 / 2}$ & $\mathrm{Fe}^{3+}$ & $712 \mathrm{eV}$ & 0.22 \\
\hline $\mathrm{Fe} 2 \mathrm{p}_{1 / 2}$ & $\mathrm{Fe}^{3+}$ & $725 \mathrm{eV}$ & 0.22
\end{tabular}

Overall, the complexity of the Fe peak fits and the limited spectral resolution due to time constraint on hiring the instrument, limit the accuracy of the compositional quantification. However, the results presented below still illustrate the relative changes in elemental composition with milling time or, analogously, depth.

Figure 4.22 shows the normalised tellurium, selenium, oxygen, and iron concentration after different milling times for the as-grown (a) and air-annealed (b) sample. For the as-grown sample, all changes in concentration occurred below 30 minutes of milling time. Deeper into the sample, the concentration profiles level off to the composition of the as-grown $\mathrm{FeSe}_{0.35} \mathrm{Te}_{0.65}$ crystal. The oxygen concentration profile diminishes rapidly to almost zero within the first 10 minutes of milling. Interestingly, as the 

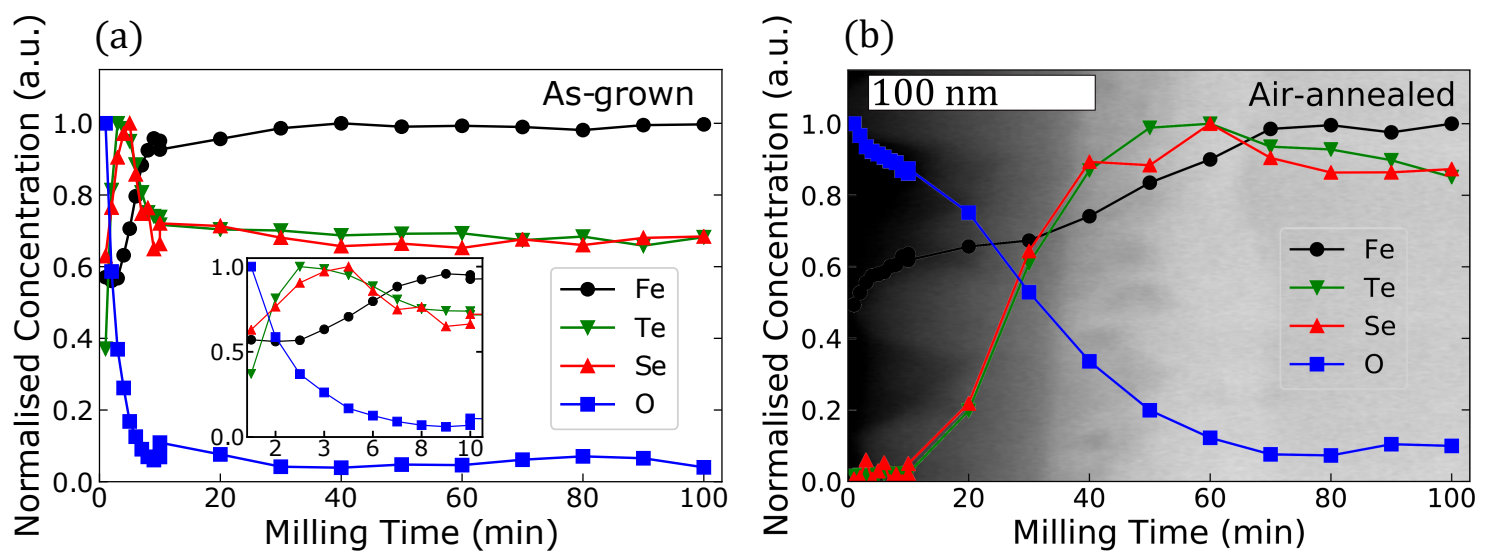

Figure 4.22: Normalised concentration profile of $\mathrm{Fe}, \mathrm{Te}, \mathrm{Se}$, and $\mathrm{O}$ obtained by XPS for the as-grown (a) and the air-annealed (b) $\mathrm{FeSe}_{0.35} \mathrm{Te}_{0.65}$ single crystal. The background in (b) shows the scaled cross-sectional STEM image of figure 4.14 .

oxygen concentration decreases, both the tellurium and selenium concentration go through a maximum that coincides with a reduced iron concentration compared to deeper inside the sample. This suggests that there is a layer beneath the oxide layer, where the tellurium and selenium to iron ratio is highest or in other words the amount of iron is reduced compared to the bulk of the sample. These results match those obtained from the SEM and STEM analysis presented in section 4.4.2 and 4.5, respectively. The iron in the sub-surface region was drawn out to react with oxygen creating an iron-deficient or equivalently tellurium- and selenium-rich layer immediately underneath the iron oxide layer.

Even though the elemental concentration profiles of the air-annealed sample might, at first glance, appear different compared to the as-grown state, they actually exhibit similar changes in concentration just on a different depth scale. This similarity is best illustrated by the enlargement of the concentration profile of the as-grown sample for milling times below 10 minutes shown in the inset of figure 4.22 (a). The main difference between the two lies in the penetration depth of the oxygen and the resulting changes in the other elemental concentrations. During the air annealing of the sample at elevated temperatures, the oxygen diffuses deeper into the sample and consequently concentration changes of iron, tellurium and selenium are also stretched 
to higher milling times.

The oxygen concentration is again largest closest to the surface and continuously decreases with increasing milling time to an approximately constant level after $70 \mathrm{~min}$ of milling. As suggested by the peak position of the Fe2p peaks, the oxygen in this region reacted with iron to form iron oxides. In fact, in the surface region below 10 minutes of milling, there is no tellurium or selenium, which agrees with the formation of the blue iron oxide surface layer detected by Raman analysis above. Both the tellurium and selenium concentration exhibit a broad maximum around $50 \mathrm{~min}$ of milling at which the iron level is still increasing. In analogy to the asgrown sample, this suggests a region with reduced iron concentration compared to the bulk. Beyond 60 min of milling, the concentrations of all detected elements start to plateau. This is again similar to what was observed in the as-grown sample.

Overall, both the as-grown and air-annealed sample suggest an area with reduced iron concentration compared to the bulk of the sample just underneath the surface iron oxide layer. This aligns well with the results from the SEM and STEM analysis shown in section 4.4 .2 and 4.5 . To further illustrate the conformity, the STEM image of the previous section has been superimposed as the background of the XPS graph of figure 4.22 (b). The changes in Z-contrast of the STEM image align nicely with the changes in the Te, Se, Fe and $\mathrm{O}$ concentrations as determined by XPS.

\subsection{Theoretical considerations on the iron oxide layer thick- ness}

\subsubsection{Constructive surface-interface interference}

The experimental investigation of air-annealed $\mathrm{FeSe}_{1-x} \mathrm{Te}_{x}$ single crystals has shown that air annealing leads to the formation of iron oxide on any exposed surface. As a result, the surface colour changes from black in the as-grown state to blue after air annealing. This was observed for all samples investigated in this work and has also 


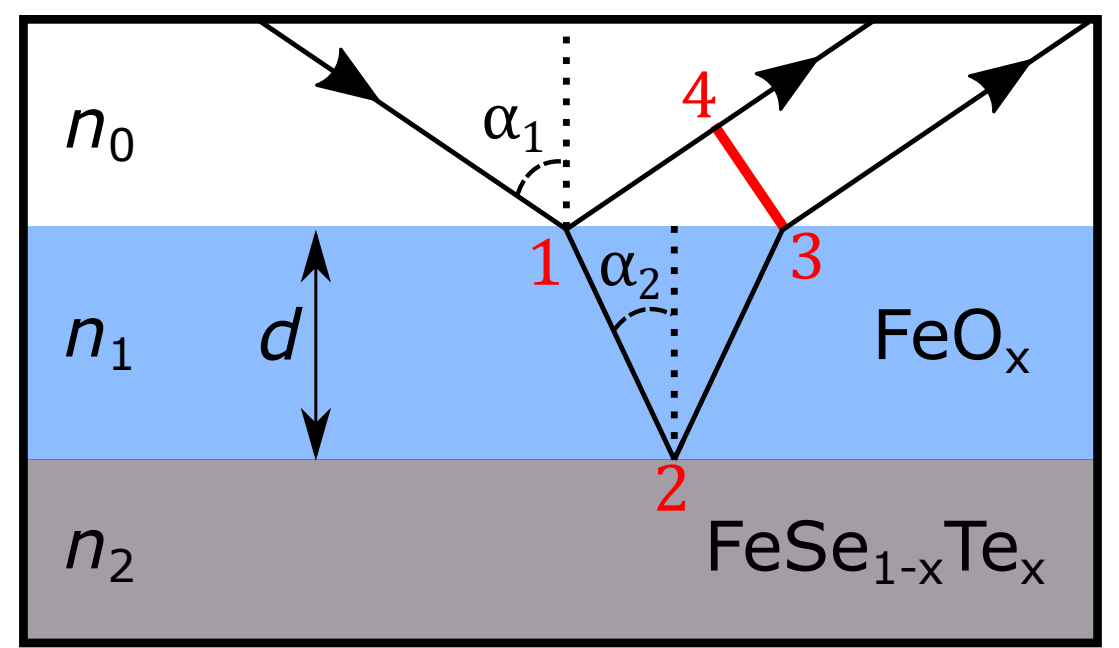

Figure 4.23: Schematic of the proposed constructive interference at the surface of the air-annealed $\mathrm{FeSe}_{1-x} \mathrm{Te}_{x} . n_{0}$ is the refractive index of air, $n_{1}$ the refractive index of the iron oxide layer and $n_{2}$ the refractive index of $\mathrm{FeSe}_{1-x} \mathrm{Te}_{x}$. The dashed lines serve as visual aid for the refraction angles $\alpha_{1}$ and $\alpha_{2}$.

been reported by several authors [Don11, Sun14, Don19]. However, iron oxide itself is not blue. Consequently, the blue colour is likely to be the result of constructive interference at the surface interface from which one can estimate the thickness of this oxide layer.

Figure 4.23 shows a simplified sketch of the surface region of an air-annealed $\mathrm{FeSe}_{1-x} \mathrm{Te}_{x}$ single crystal that illustrates the constructive interference of the incoming light. In general, constructive interference occurs when the path difference, $\Delta s$, is an integer multiple of the wavelength of the incident light, $\lambda$. As illustrated by the schematic diagram in figure $4.23, \Delta s$ is given by

$$
\Delta s=n_{1}\left(s_{12}+s_{23}\right)-n_{0} s_{14}
$$

where $s_{12}$ is the path from 1 to $2, s_{23}$ is the path from 2 to $3, s_{14}$ is the path from 1 to 4 , and $n_{0}$ and $n_{1}$ are the refractive indices of air and $\mathrm{FeO}_{x}$, respectively. The individual paths are given as

$$
s_{12}=s_{23}=\frac{d}{\cos \left(\alpha_{2}\right)}
$$


and

$$
s_{14}=2 d \tan \left(\alpha_{2}\right) \sin \left(\alpha_{1}\right)
$$

where $\alpha_{1}$ and $\alpha_{2}$ are the reflection angles at the respective surface shown in figure 4.23 and $d$ is the thickness of the oxide layer. Substituting $s_{12}, s_{23}$ and $s_{14}$ into equation 4.1 and inserting Snell's law, $n_{1} \sin \left(\alpha_{1}\right)=n_{2} \sin \left(\alpha_{2}\right)$ leads to

$$
\begin{aligned}
\Delta s & =2 n_{1} d / \cos \left(\alpha_{2}\right)-\left(2 n_{0} d / \tan \left(\alpha_{2}\right) \sin \left(\alpha_{1}\right)\right. \\
& =2 n_{1} d\left(1 / \cos \left(\alpha_{2}\right)-\tan \left(\alpha_{2}\right) \sin \left(\alpha_{1}\right)\right) \\
& =2 n_{1} d / \cos \left(\alpha_{2}\right)\left(1-\sin ^{2}\left(\alpha_{2}\right)\right) \\
& =2 n_{1} d \cos \left(\alpha_{2}\right)
\end{aligned}
$$

For an incident angle of $0^{\circ}$, the condition for constructive interferences simplifies to

$$
\Delta s=m \lambda=2 n_{1} d
$$

where $\lambda$ is the wavelength of the incident light, and $m$ any positive integer greater than 0. Given that the crystal's surface appears blue, the condition must be fulfilled for blue light, which wavelength is in the range of $400 \mathrm{~nm}$ to $500 \mathrm{~nm}$ (from dark blue to light blue). The Raman analysis in section 4.3 has shown that the surface layer predominantly consists of magnetite and hematite. Assuming a full magnetite layer, with the refractive index of magnetite being $n_{1}=2.42$, the thickness of the magnetite layer, $d$, can be estimated using the condition for constructive interference

$$
d=\frac{m \lambda}{2 n_{1}}
$$

For the first harmonic, $m=1$, this leads to a thickness between $82 \mathrm{~nm}$ and $103 \mathrm{~nm}$ from light blue to dark blue. This simple estimate agrees well with the thickness that was obtained by the SEM, STEM, and XPS analysis. 


\subsubsection{Estimate of the iron oxide thickness for complete removal of the interstitial excess iron}

In this section, a 'Gedankenexperiment' (English: thought experiment) that relates the thickness of an iron oxide layer to the amount of (interstitial) excess iron that has been drawn out from the bulk of the sample is proposed:

If (air) annealing were to fully remove all excess iron from a crystal, what would the thickness of the resulting iron oxide layer be?

The number of Fe atoms in a $\mathrm{FeSe}_{1-x} \mathrm{Te}_{x}$ single crystal, $N_{\text {sample, }}^{\mathrm{Fe}}$ is related to the volume of the sample as follows

$$
N_{\text {sample }}^{\mathrm{Fe}}=N_{\text {unit,FeSeTe }}^{\mathrm{Fe}} \frac{V_{\text {sample }}}{V_{\text {unit,FeSeTe }}},
$$

where $N_{\text {unit,FeSeTe }}^{\mathrm{Fe}}$ is the number of iron atoms per unit cell of $\mathrm{FeSe}_{1-x} \mathrm{Te}_{x}, V_{\text {unit,FeSeTe }}$ the volume of the unit cell, and $V_{\text {sample }}$ the total sample volume. Assuming that the excess iron does not greatly alter the volume of the unit cell, it can be estimated by

$$
N_{\text {excess }}^{\mathrm{Fe}}=R_{\text {excess }} N_{\text {sample }}^{\mathrm{Fe}}
$$

with $R_{\text {excess }}$ being the relative amount of excess iron. In analogy to equation 4.7 , the amount of iron in the iron oxide surface layer is related to the volume by

$$
N_{\mathrm{FeO} x}^{\mathrm{Fe}}=N_{\text {unit, } \mathrm{FeO}_{x}}^{\mathrm{Fe}} \frac{V_{\mathrm{FeO}_{x}}}{V_{\mathrm{unit}_{\text {, } \mathrm{FeO}}}},
$$

where $N_{\mathrm{unit}}^{\mathrm{Fe}}, \mathrm{FeO}_{x}$ is the number of iron atoms per unit cell of $\mathrm{FeO}_{x}, V_{\text {unit, } \mathrm{FeO}_{x}}$ the volume of a unit cell and $V_{\mathrm{FeO}_{x}}$ the volume of the iron oxide layer. For complete removal of excess iron within the sample, the amount of excess iron in the as-grown sample has to equal the amount of $\mathrm{Fe}$ in the oxide, $N_{\text {excess }}^{\mathrm{Fe}}=N_{\mathrm{FeO}_{x}}^{\mathrm{Fe}}$. Plugging in equation 4.8 and 4.9 and solving for the volume of the oxide layer leads to 


$$
V_{\mathrm{FeO}_{x}}=R_{\text {excess }} V_{\text {sample }} \frac{V_{\text {unit,FeO }} x}{V_{\text {unit,FeSeTe }}} \frac{N_{\text {unit }}^{\mathrm{Fe}}}{N_{\text {unit, } \mathrm{FeO}_{x}}^{\mathrm{Fe}}}
$$

The amount of excess iron in as-grown $\mathrm{FeSe}_{1-x} \mathrm{Te}_{x}$ single crystals has been reported to vary between $2 \%$ and $14 \%$ [Bao09, Vie10, Hu11, Sun14, Sun14, Sun19]. This variance is a result of different synthesis conditions and different characterisation methods [Bao09, Vie10, Hu11, Sun14, Sun14, Sun19]. Assuming the formation of only hematite and magnetite, as was shown in section 4.3 , the theoretical iron oxide volume is estimated for the two boundary cases of $2 \%$ and $14 \%$ excess iron and the formation of $100 \%$ hematite and $100 \%$ magnetite. Table 4.4 shows the estimated volume for all four cases as percentage of the total sample volume and the resulting thickness based on typical sample dimensions of $1 \mathrm{~mm} \times 1 \mathrm{~mm} \times 0.1 \mathrm{~mm}$, assuming the oxidation is confined to the bottom and top surfaces.

Table 4.4: Relative volume of the iron oxide layer and thickness for typical sample dimensions for $2 \%$ and $14 \%$ excess iron for the formation of magnetite and hematite.

\begin{tabular}{r|c|c|c|c} 
Excess Iron & $2 \%$ & $2 \%$ & $14 \%$ & $14 \%$ \\
\hline Iron Oxide & Magnetite & Hematite & Magnetite & Hematite \\
\hline Relative iron oxide volume[\%] & 1.1 & 1.2 & 7.9 & 8.1 \\
\hline Thickness [nm] & 570 & 580 & 3950 & 4050
\end{tabular}

This simple estimation immediately shows that full removal of the excess iron by oxidation of only the top- and bottom surfaces is highly unlikely. Even in the case of just $2 \%$ initial excess iron, the surface layer would have to be about $600 \mathrm{~nm}$ thick, which is 6 times the thickness of the experimentally determined thickness by SEM, STEM, XPS, and the interference analysis discussed above. The combination of the two results therefore suggests that full removal of excess iron is only feasible when the oxidation of the sample is not limited to the top and bottom surface but any surface that is exposed to oxygen as suggested by the microscopy images in figures 4.6 and 4.7. Having said that, it is unclear if it is actually necessary to fully remove the excess iron to improve the superconducting properties of $\mathrm{FeSe}_{1-x} \mathrm{Te}_{x}$. However, 
a reduction in the excess iron concentration by a mere $0.35 \%$, as would be the case for top- and bottom surface oxidation only, does seem unlikely to have a significant effect on the superconducting properties of the whole $\mathrm{FeSe}_{1-x} \mathrm{Te}_{x}$ crystal.

\subsection{Diffusion model}

Based on the results presented above, a diffusion model is proposed to explain the observed formation of an iron oxide surface layer as well as the resulting reduction of excess iron within the sample. The general idea of the model is depicted at different characteristic times in figure 4.24 for the case of annealing in an oxygen-containing atmosphere. The figure shows three two-dimensional $\mathrm{FeSe}_{1-x} \mathrm{Te}_{x}$ layers with gaps in the layer to symbolise lattice defects such as grain boundaries, cracks or twinboundaries. The inclusion of these defects is necessary to account for the formation of, for example, the web-like structures on the surface as presented in section 4.2 . The spheres represent the different elements, that is, iron (brown), selenium and tellurium (blue), interstitial iron (red), and the oxygen of the annealing atmosphere (green). The dashed red circles represent previously occupied interstitial (iron) sites. The grey background qualitatively illustrates the interstitial iron concentration from high to low as grey to white, respectively.

At $t=t_{0}$, the sample is at room temperature in the initial as-grown state and a substantial amount of interstitial iron is distributed throughout the sample. When the temperature is increased $\left(t=t_{1}\right)$, the interstitial excess iron close to the surface starts to react with oxygen to form iron oxides. This leads to a reduction in the iron concentration near the surface and results in an iron concentration gradient that drives more interstitial excess iron from deeper in the sample towards the surface. At $300^{\circ} \mathrm{C}$, which is the temperature that all the samples in this study are annealed at, this process continues to draw more excess iron from inside the sample and the iron oxide surface layer continues to grow as depicted at $t=t_{2}$. At $t=t_{\text {final }}$, the $\mathrm{FeO}_{x}$ layer has become thick enough to act as a diffusion barrier preventing the 

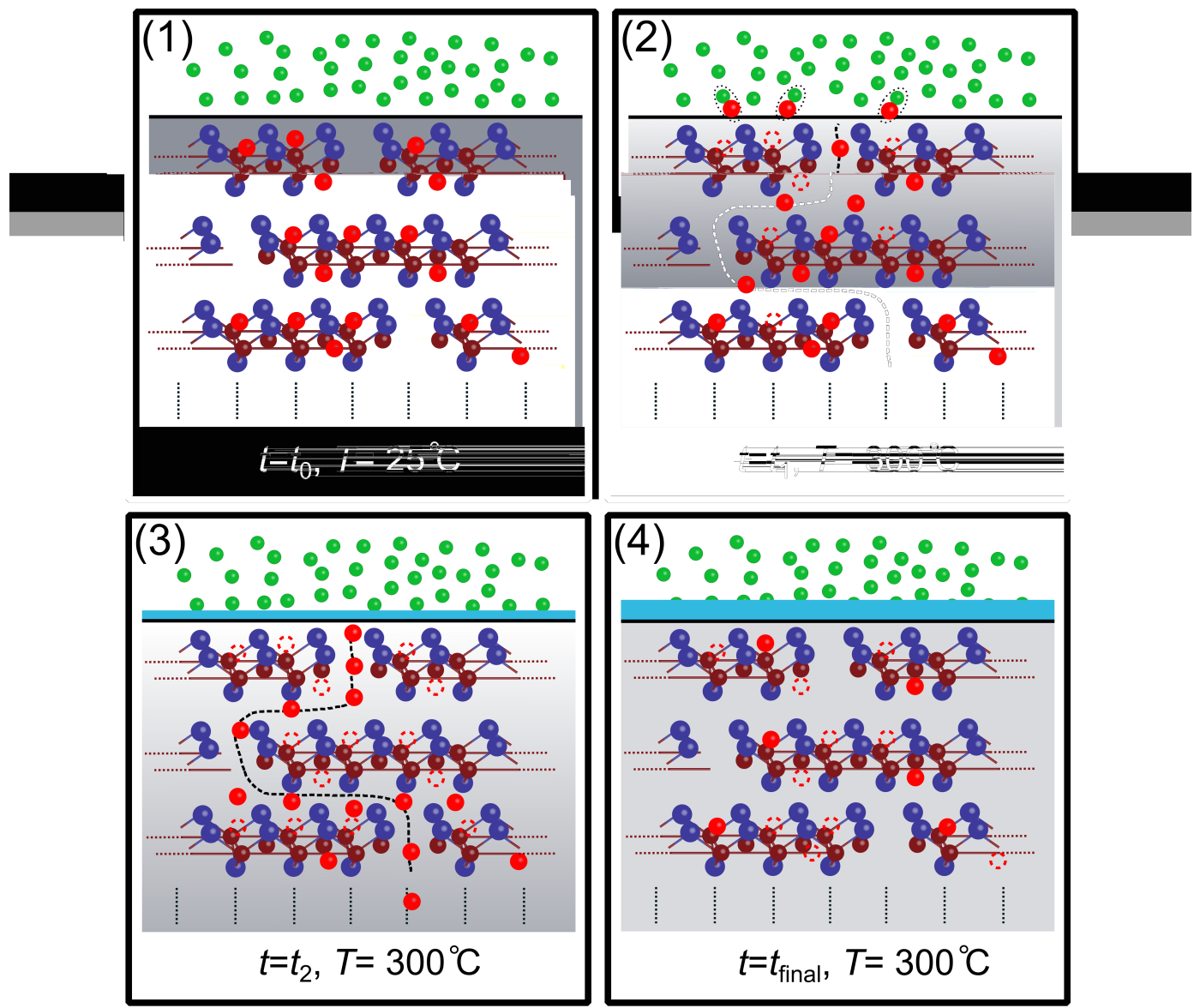

- Selenium/Tellurium • Lattice Iron •Interstitial Iron • Oxygen $=$ Iron Oxide $100 \%$

$0 \%$

interstitial iron concentration

Figure 4.24: Schematic drawing of the proposed model for the diffusion of interstitial iron towards the surface. The schematics depict four characteristic stages at different times in the annealing process: (1) before annealing, (2) the start of the annealing process, (3) after a certain elapsed time $\left(t_{2}>t_{1}\right)$, and lastly (4) after the annealing. The grey background symbolises the interstitial excess iron concentration at the respective depth: the darker the grey, the higher the iron concentration and vice versa.

external oxygen from further reacting with the iron on the exposed surfaces of the sample. The remaining interstitial excess iron within the sample cannot react with the oxygen and the concentration gradient, hence the chemical potential, starts to decrease as the sample reaches a steady state. In this final state, the interstitial iron is again uniformly distributed throughout the surface region of the sample, but the 
total amount of interstitial excess iron within this surface region is depleted due to the formation of the iron oxide on the surface. This is the iron depleted subsurface layer as presented in the STEM and XPS images and plots.

In this model, the diffusion of the interstitial iron is characterised by two diffusion paths: in-plane diffusion and inter-plane diffusion. In-plane diffusion is expected to be very fast due to the weakly-bound two-dimensional layered structure of $\mathrm{FeSe}_{1-x} \mathrm{Te}_{x}$, however, inter-plane diffusion through the layers is not. Therefore, the preferential diffusion through defects that act as 'fast paths' determine the interplane diffusion [Lau14. Specifically, the iron atoms diffuse in-plane until they reach a defect that allows for fast inter-plane diffusion [Lau14. These preferential diffusion paths align with the formation of patterns on the surface as shown in figures 4.3, 4.4, and 4.5. Overall, the key requirement for our model is that the annealing atmosphere contains an element for the excess iron to react with during annealing. The iron reactant could be oxygen, as in our case, or other elements that form iron compounds. This is consistent with the observed formation of iron compounds and the related improvement in the superconducting properties when annealing in $\mathrm{Te}$ [Cha05, Pra16], Se [Oka11, S Oka11] or O [Sun19], air [Don11], and, based on the results presented in chapter 6, also in the case of $\mathrm{N}_{2}$ or low vacuum annealing due to the presence of residual oxygen.

\subsection{Conclusion}

In this chapter, the effect of air annealing on the structural properties and chemical composition of $\mathrm{FeSe}_{1-x} \mathrm{Te}_{x}$ single crystals was presented. Based on optical microscopy and Raman spectroscopy, it was shown that iron oxide forms not only on the top- and bottom surface but any surface exposed to air during annealing. The thickness of this surface iron oxide layer was found to be on the order of $100 \mathrm{~nm}$. Additionally, it was shown that an iron-deficient layer of similar thickness is formed underneath the iron oxide layer using cross-sectional SEM, STEM and depth-profiling 
XPS analysis. To support the experimental findings, theoretical considerations on the iron oxide thickness and the associated excess iron reduction were presented. These considerations reinforce an iron oxide layer on the order of $100 \mathrm{~nm}$ and furthermore illustrate that the oxidation of the top and bottom surface alone do not suffice to substantially reduce the amount of excess in the sample. Lastly, a diffusion model was proposed to explain the change in surface composition and the formation of structures such as the web-like structure and the iron oxide pockets. 



\section{Effect of air annealing on the superconducting properties of $\mathrm{FeSe}_{1-x} \mathrm{Te}_{x}$}

In this chapter, the effect of air annealing on the superconducting properties of $\mathrm{FeSe}_{1-x} \mathrm{Te}_{x}$ single crystals is discussed. First, zero-field-cooled (ZFC) and field-cooled (FC) magnetic susceptibility and magnetic hysteresis loop measurements of four differently doped $\mathrm{FeSe}_{1-x} \mathrm{Te}_{x}$ samples in the as-grown state and after air annealing are presented. The observed changes in the superconducting properties are then linked to the changes in the surface chemistry and structure as presented in chapter 4. In the last section of this chapter, the superconducting properties of a stepwise exfoliated, air-annealed $\mathrm{FeSe}_{0.35} \mathrm{Te}_{0.65}$ single crystal are investigated. The aim of this later work is to examine if the largest excess iron reduction in the near-surface region as shown in section 4.6 also translates into the largest improvement in the superconducting properties in that region compared to the bulk of the sample.

\subsection{ZFC-FC magnetisation of as-grown and air-annealed $\mathrm{FeSe}_{1-x} \mathrm{Te}_{x}$}

Figure 5.1 shows the demagnetisation-corrected ZFC-FC magnetic susceptibility of four differently doped $\mathrm{FeSe}_{1-x} \mathrm{Te}_{x}$ single crystals in the as-grown state and after air annealing. To take sample geometry- and size-dependent demagnetisation effects into account, the magnetic susceptibility was corrected following Prozorov et al. as described in detail in experimental section 3.4.1 [Pro18].

Starting with the as-grown state of the sample with the lowest selenium concentration, $\mathrm{FeSe}_{0.15} \mathrm{Te}_{0.85}$ (see figure 5.1(a)), the ZFC- and FC-susceptibility are indistinguishable and exhibit no diamagnetic shielding. This insinuates that the as- 
(a)

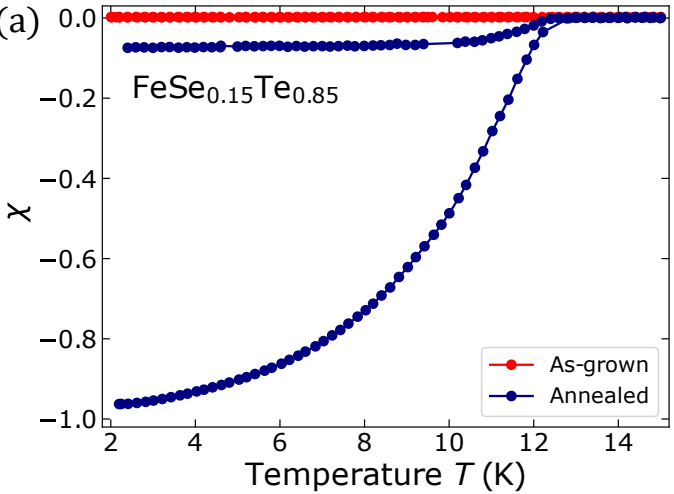

(c)

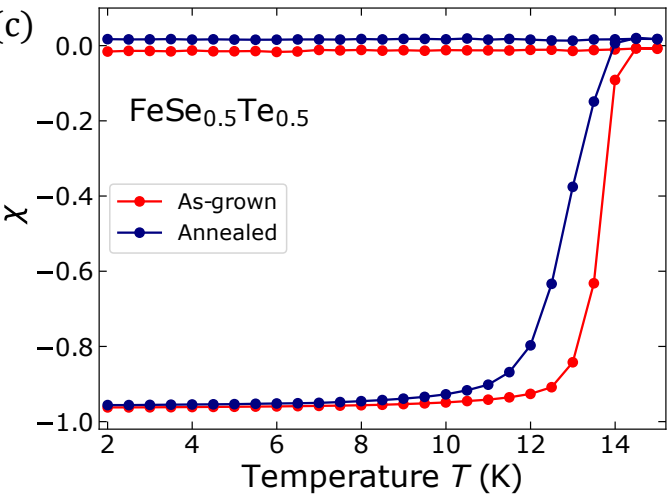

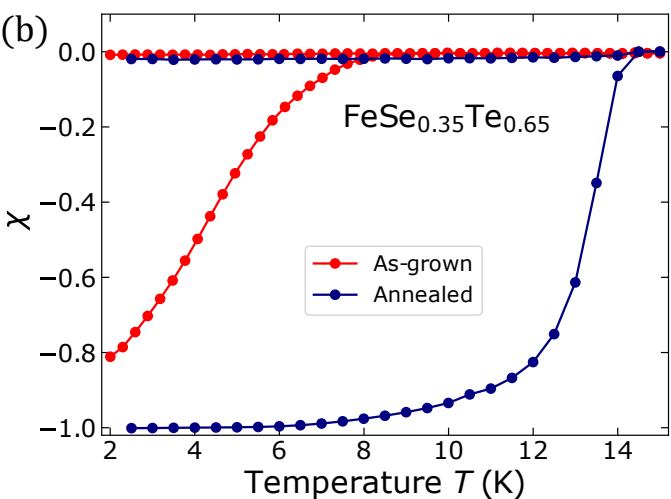

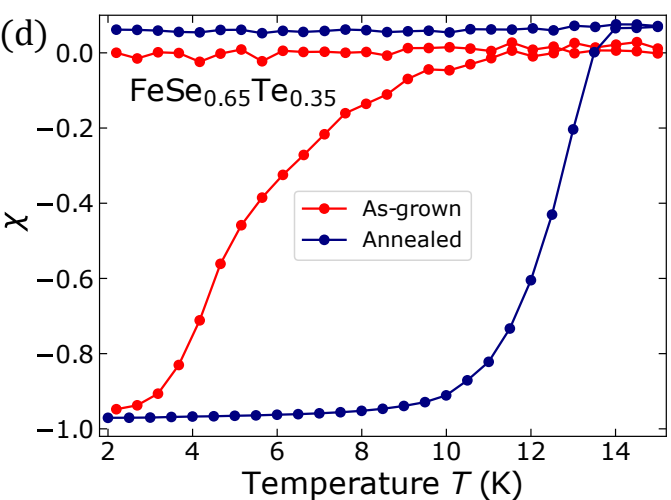

Figure 5.1: Plot of the zero-field-cooled (ZFC) and field-cooled (FC) magnetic susceptibility against temperature for as-grown and air-annealed $\mathrm{FeSe}_{1-x} \mathrm{Te}_{x}$ single crystals with $\mathrm{x}=\{0.85(\mathrm{a}), 0.65(\mathrm{~b}), 0.5(\mathrm{c}), 0.35(\mathrm{~d})\}$. The susceptibility was corrected for demagnetisation effects following Prozorov et al. [Pro18].

grown state of the $\mathrm{FeSe}_{0.15} \mathrm{Te}_{0.85}$ crystal is not superconducting, which aligns with reports of similarly doped as-grown crystals from other studies in the literature Noj10, Hu12a, Kaw12. It is only after air annealing that superconductivity is induced with $T_{\mathrm{c}}=12.8 \mathrm{~K}$. However, the superconducting transition remains very broad with the susceptibility just starting to plateau at $2 \mathrm{~K}$ and not exhibiting full diamagnetic shielding at the lowest measured temperature, $\chi(2 \mathrm{~K})>-1$. Furthermore, in contrast to the other dopings, the FC magnetic susceptibility also exhibits a superconducting transition with a diamagnetic signal of $\chi(2 \mathrm{~K}) \approx-0.1$. This diamagnetic signal is the result of the Meissner effect that leads to the expulsion of magnetic flux from the superconductor (see section 2.1.2). However, the Meissner effect is typically suppressed in dirty type II superconductor due to the pinning of the magnetic flux lines. More specifically for strong pinning and consequently large $J_{\mathrm{c}}$, the Meissner 
effect is essentially fully suppressed [Cle93, Cam91]. This is for example the case for all other dopings. Thus, the appearance of a Meissner effect and a diamagnetic susceptibility of $\chi(2 \mathrm{~K}) \approx-0.1$ insinuates weak flux pinning and potentially a small $J_{\mathrm{c}}$ for this doping.

The $\mathrm{FeSe}_{0.35} \mathrm{Te}_{0.65}$ sample (figure 5.1(b)) already exhibits a superconducting transition in the as-grown state. However, the fact, that the magnetic susceptibility has not plateaued at $2 \mathrm{~K}$ and only reaches a value of $\chi(2 \mathrm{~K}) \approx-0.8$, suggests that the sample is not fully superconducting. Furthermore, the broad superconducting transition, which stretches from $T_{\mathrm{c}}=9 \mathrm{~K}$ to below the lowest measured temperature of $2 \mathrm{~K}$, indicates the presence of weak links or that the sample is not homogeneously superconducting, that is, $T_{\mathrm{c}}$ varies throughout the sample. After air annealing, $T_{\mathrm{c}}$ increased to $14 \mathrm{~K}$, the transition width decreased, and the sample shows perfect diamagnetic shielding $\chi(2 \mathrm{~K}) \approx-1$.

Similar behaviour to the $\mathrm{FeSe}_{0.35} \mathrm{Te}_{0.65}$ sample is observed in the $\mathrm{FeSe}_{0.65} \mathrm{Te}_{0.35}$ sample shown in figure 5.1(d). The as-grown sample exhibits a very broad transition, a low $T_{\mathrm{c}}$, and the magnetic susceptibility just barely starts to plateau at $2 \mathrm{~K}$. After annealing, the transition is sharpened, $T_{\mathrm{c}}$ is increased to $13.5 \mathrm{~K}$, and the sample shows full diamagnetic shielding from around $8 \mathrm{~K}$ downwards.

In contrast to the three aforementioned samples, the optimally doped sample, $\mathrm{FeSe}_{0.5} \mathrm{Te}_{0.5}$, already displays a sharp transition, a high $T_{\mathrm{c}}$, and full diamagnetic shielding in the as-grown state. In fact, at this doping, the superconducting transition is slightly broadened and $T_{\mathrm{c}}$ slightly decreased after air annealing. However, the air-annealed sample still exhibits a constant diagmagnetic susceptibility just short of $\chi(2 \mathrm{~K}) \approx-1$ similar to the as-grown state. Given the almost constant diamagnetic response in the temperature range from $6 \mathrm{~K}$ to $2 \mathrm{~K}$, it is safe to assume that the sample actually exhibits perfect diamagnetism in both the as-grown and air-annealed state. The deviation from -1 is likely to be the result of demagnetisation and the related uncertainty of its correction [Pro18]. The slight broadening of the transition after air-annealing suggests the formation of weak links or defects and will be further 


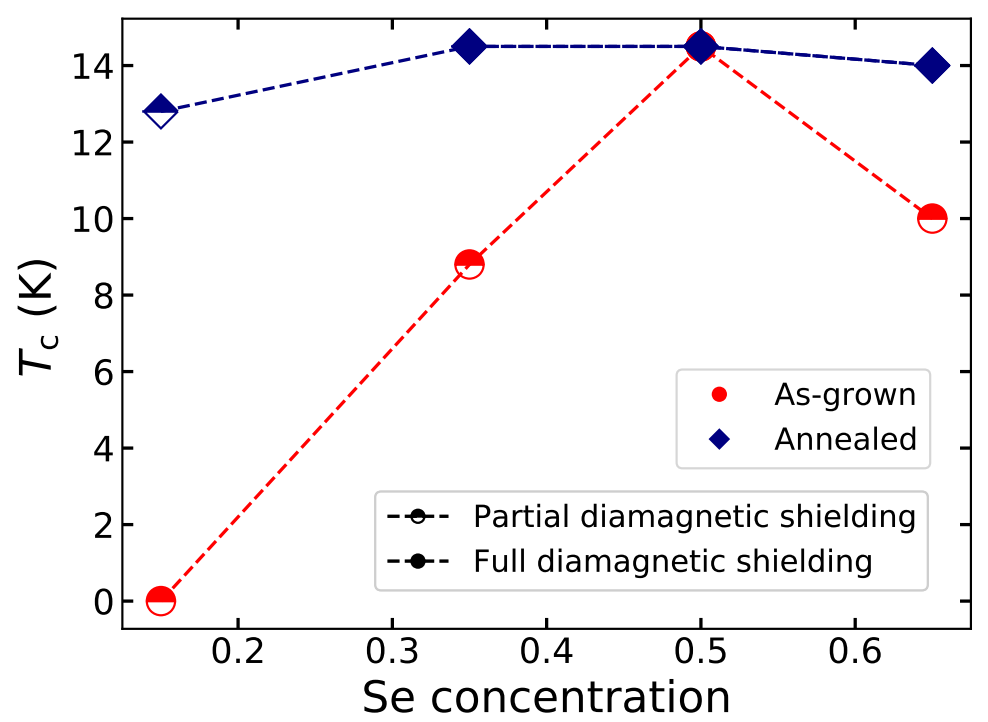

Figure 5.2: Critical temperature, $T_{\mathrm{c}}$, versus selenium concentration for as-grown (red spheres) and air-annealed (blue diamonds) $\mathrm{FeSe}_{1-x} \mathrm{Te}_{x}$. Samples exhibiting full diamagnetic shielding $(\chi \approx-1)$ are plotted as solid symbols and samples that only show partial diamagnetic shielding $(\chi>-1)$ are plotted as half-filled symbols.

analysed in the context of $J_{\mathrm{c}}$ in the following section 5.2 . Overall, the behaviour of the optimally doped sample is in contrast to all other dopings, for which only the air-annealed samples exhibit full diamagnetic shielding.

The critical temperatures of the four analysed selenium concentrations are summarised in figure 5.2 for the as-grown and air-annealed state. Before air annealing, the critical temperature between the different doping levels varied from nonsuperconducting to $14.5 \mathrm{~K}$. Furthermore, only the optimally doped samples exhibited perfect diamagnetic shielding. All other samples were not fully superconducting in the as-grown state. After air annealing, $T_{\mathrm{c}}$ of all samples, except for the optimally doped sample, increased and the variance in $T_{\mathrm{c}}$ between the differently doped samples was reduced to $12.8 \mathrm{~K}$ to $14.5 \mathrm{~K}$. Moreover, samples of all doping levels, except $\mathrm{FeSe}_{0.15} \mathrm{Te}_{0.85}$ which is just starting to plateau, exhibit full diamagnetic shielding in the air-annealed state.

In the previous chapter, it was shown that air annealing leads to the formation of iron oxide on any exposed surface (see for instance figure 4.7). During this forma- 
tion, interstitial excess iron, which suppresses superconductivity in this family of superconductors McQ09, Liu09, Zha09, Don11, is withdrawn from the $\mathrm{FeSe}_{1-x} \mathrm{Te}_{x}$ phase. As a result, the superconducting transition width, $T_{\mathrm{c}}$, and the diamagnetic shielding of almost all samples is improved after air-annealing.

\subsection{MHLs and $J_{c}$ of as-grown and air-annealed FeSe $_{1-x} \mathbf{T e}_{x}$}

Figure 5.3 shows the background-corrected magnetic hysteresis loops (MHLs) at $2.2 \mathrm{~K}$ for the same differently doped samples in the as-grown state and after air annealing. To reduce the background signal from the sample capsule and other potential parasitic contributions, the background was corrected following the procedure introduced in experimental section 3.4.2.

Besides the $\mathrm{FeSe}_{0.15} \mathrm{Te}_{0.85}$ sample, all samples exhibit essentially symmetric MHLs in the as-grown state and after air annealing. However, the MHLs of the as-grown $\mathrm{FeSe}_{0.35} \mathrm{Te}_{0.65}$ and $\mathrm{FeSe}_{0.65} \mathrm{Te}_{0.35}$ samples are small and close before reaching the highest applied magnetic field. After air annealing, the size of the hysteresis loops is greatly increased and even at the highest applied field, the magnetisation remains almost constant for the $\mathrm{FeSe}_{0.35} \mathrm{Te}_{0.65}$ sample and even increases slightly for the $\mathrm{FeSe}_{0.65} \mathrm{Te}_{0.35}$ sample. The latter is known as the second-magnetisation peak or 'fishtail effect' [Jir97]. The optimally doped sample, $\mathrm{FeSe}_{0.5} \mathrm{Te}_{0.5}$, already exhibits a large hysteresis loop up to the highest measured field in the as-grown state. However, the width of the loop is still slightly increased after air annealing.

Overall, the results from the MHL measurements of $\mathrm{FeSe}_{0.35} \mathrm{Te}_{0.65}$ and $\mathrm{FeSe}_{0.65} \mathrm{Te}_{0.35}$ are in good agreement with the ZFC-FC susceptibility measurements presented in the previous section 5.1. The as-grown samples are only weakly superconducting, that is a low $T_{\mathrm{c}}$, large transition width, and only partial diamagnetic shielding. This insinuates that the samples are not fully superconducting and only small parts of the sample contribute to the magnetic signal. As a result, both samples only exhibit small hysteresis loops in the as-grown state. After air annealing, $T_{\mathrm{c}}$ is increased, the 
(a)

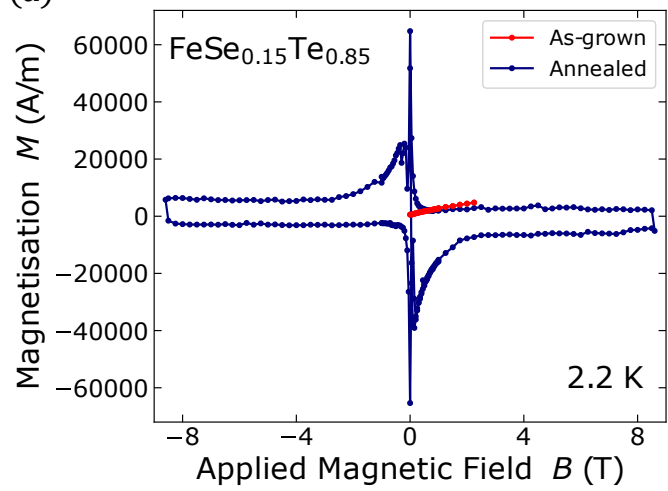

(c)

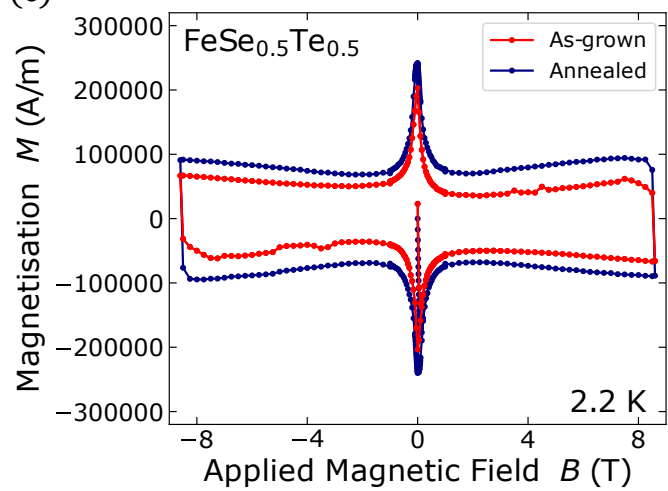

(b)

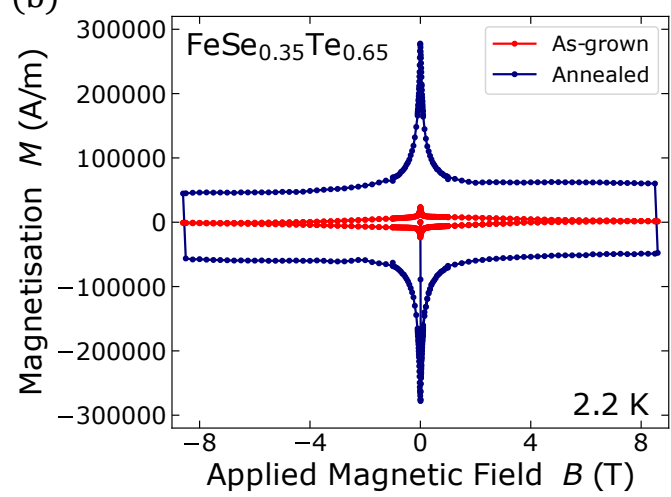

(d)

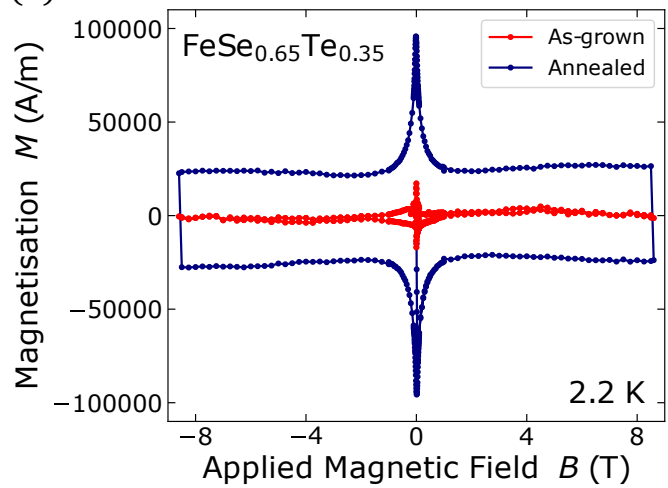

Figure 5.3: Magnetic hysteresis loops of as-grown and air-annealed $\mathrm{FeSe}_{1-x} \mathrm{Te}_{x}$ single crystals for four different dopings, $\mathrm{x}=\{0.85(\mathrm{a}), 0.65(\mathrm{~b}), 0.5(\mathrm{c}), 0.35(\mathrm{~d})\}$, taken at $2.2 \mathrm{~K}$. The magnetisation was corrected using the background correction introduced in section 3.4 .2 .

transition width is reduced, and the samples show full diamagnetic shielding. These changes together with the large increase in the size of the magnetic hysteresis loops suggest an increase in the superconducting volume fraction of both $\mathrm{FeSe}_{0.35} \mathrm{Te}_{0.65}$ and $\mathrm{FeSe}_{0.65} \mathrm{Te}_{0.35}$ after air annealing. One could also argue that the increase in the magnetisation is the result of increased pinning. However, given the fact that the magnetisation increased by more than one order of magnitude, which is considerably larger than any increase achieved by the introduction of artificial pinning centres in this compound [Eis17, Tam12, increased pinning as sole reason seems unlikely.

Interestingly, air annealing, which had a negative effect on the ZFC-FC susceptibility of $\mathrm{FeSe}_{0.5} \mathrm{Te}_{0.5}$, still has a positive effect on the MHL. Since the ZFC-FC measurements do not suggest an increase in the superconducting volume fraction after air 
annealing and given that the increase is smaller than in the case of $\mathrm{FeSe}_{0.35} \mathrm{Te}_{0.65}$ and $\mathrm{FeSe}_{0.65} \mathrm{Te}_{0.35}$, the increase in magnetisation in this case is more likely to be driven by an increase in pinning. This also aligns with the fact that the increase is not as large and more comparable to increases obtained by introduction of artificial pinning centres [Eis17, Tam12].

Coming back to the underdoped $\mathrm{FeSe}_{0.15} \mathrm{Te}_{0.85}$ sample, the as-grown sample has been shown to be non-superconducting in the previous section and therefore does not exhibit a diamagnetic signal in the MHL measurement. After air annealing, the sample shows a diamagnetic signal at low fields and a superconducting hysteresis loop. However, the size of the magnetic hysteresis loop is considerably smaller for this sample compared to the other samples with different selenium concentrations. This is in agreement with the weak pinning as suggested by the diamagnetic signal in the FC susceptibility measurement (see figure 5.1(a)). Moreover, contrary to the other samples, the MHL is not symmetric for increasing and decreasing applied magnetic field, but almost perfectly asymmetric. More specifically, the magnetisation for decreasing applied magnetic fields is largely reduced to almost zero at both positive and negative fields compared to the case of increasing fields. On a closer look, a small asymmetry in the MHL can also be seen in the as-grown $\mathrm{FeSe}_{0.5} \mathrm{Te}_{0.5}$ sample. The effect might not be as large, but the magnetisation for increasing fields is slightly larger and, opposite to the magnetisation for decreasing fields, exhibits no flux jumps. The latter has also been observed in other asymmetric MHLs that are later discussed in chapter 7 in the context of a comprehensive analysis of the asymmetric MHLs.

So far, almost all research has been focused on the effect of annealing on $T_{\mathrm{c}}$, but not a lot of effort has been channelled to investigate the effect of annealing on the critical current density for differently doped $\mathrm{FeSe}_{1-x} \mathrm{Te}_{x}$ single crystal Noj10, Don11, Kaw12, Hu12a, Sun16]. Figure 5.4 presents a $J_{c}$ versus $B$ summary plot for as-grown and air-annealed $\mathrm{FeSe}_{1-x} \mathrm{Te}_{x}$ single crystals for four different selenium concentrations. Overall, air annealing the samples resulted in an increase in $J_{\mathrm{c}}$ for all doping levels $(\mathrm{x}=\{0.35,0.5,0.65,0.85\})$. For $\mathrm{x}=0.65$ and $\mathrm{x}=0.35, J_{\mathrm{c}}$ 


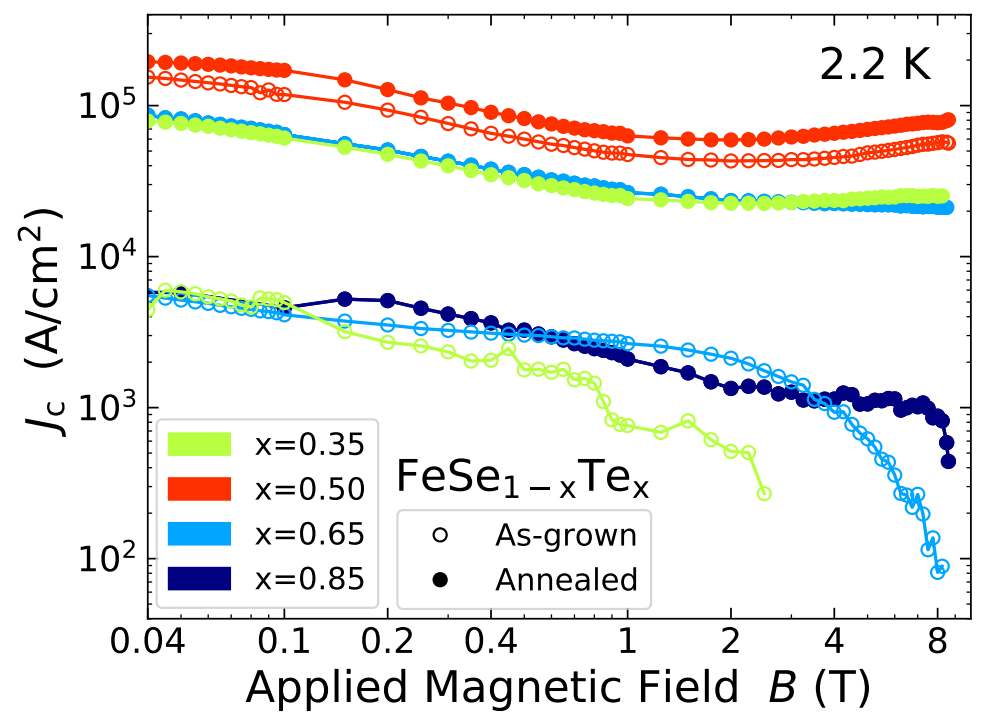

Figure 5.4: Critical current density, $J_{\mathrm{c}}$, versus applied magnetic field, $B$, of the as-grown $\mathrm{FeSe}_{1-x} \mathrm{Te}_{x}$ single crystals and after air annealing for different Se concentrations at $2.2 \mathrm{~K}$.

increased by more than one order of magnitude for all applied fields and only slowly decreases with increasing field. In fact, the $\mathrm{FeSe}_{0.65} \mathrm{Te}_{0.35}$ sample even exhibits a small second magnetisation peak with $J_{\mathrm{c}}$ increasing at high fields above $4 \mathrm{~T}$. As previously mentioned, the large increase in $J_{c}$ observed for both $\mathrm{FeSe}_{0.35} \mathrm{Te}_{0.65}$ and $\mathrm{FeSe}_{0.65} \mathrm{Te}_{0.35}$ suggests an increase in the superconducting volume fraction. Excess iron, which suppresses superconductivity McQ09, Liu09, Zha09, Don11, was extracted from the $\mathrm{FeSe}_{1-x} \mathrm{Te}_{x}$ phase by reacting with the oxygen during air annealing and thus the superconducting volume was increased. In the case of the optimally doped sample, $\mathrm{FeSe}_{0.5} \mathrm{Te}_{0.5}, J_{\mathrm{c}}$ of the as-grown sample was already relatively high, but air annealing still led to a $40 \%$ increase in $J_{\mathrm{c}}$ at all applied fields. Furthermore, similar to the air-annealed $\mathrm{FeSe}_{0.65} \mathrm{Te}_{0.35}$ sample, the sample exhibits a second magnetisation peak in the as-grown and air-annealed state with $J_{\mathrm{c}}$ increasing for fields above $4 \mathrm{~T}$. The increase in $J_{\mathrm{c}}$ after air annealing shows that even though $T_{\mathrm{c}}$ was slightly decreased (see ZFC-FC susceptibility shown in figure 5.1), $J_{c}$ can still be enhanced by air annealing. Contrary to $T_{\mathrm{c}}, J_{\mathrm{c}}$ in type II superconductors is typically not limited by the intrinsic limit of the superconductor at which the kinetic energy of the Copper pairs would exceed the condensation energy, known as depairing current, 
but rather by the combined pinning strength of the pinning centres that prevent the movement of magnetic flux (see section 2.1.7 in chapter 2). Hence, one possible explanation would be that air annealing introduced additional defects that act as flux pinning centres. For example, regions with reduced $T_{\mathrm{c}}$ or non-superconducting regions have a detrimental effect on the ZFC-magnetisations, however, they act as additional pinning centres to prevent flux movement and can increase $J_{\text {c }}$.

Another possible cause could be an increase in the superconducting volume fraction of the sample. In the case here, the as-grown sample already showed a sharp transition and a full superconducting screening fraction. However, that does not guarantee that the whole sample was superconducting. It is likely that only the surface was fully superconducting whilst the bulk was just partially superconducting. After annealing in air, the superconducting volume was increased, which consequently contributed to the observed increase in $J_{c}$. This explanation also aligns with the structural characterisation presented in the previous section. As suggested by the XPS analysis of an as-grown single crystal, atmospheric oxygen might have reacted with the iron close to the surface and hence created a layer with reduced excess iron just underneath. This reduction in excess iron on the surface created a fully superconducting layer, which led to the full diamagnetic shielding displayed in the ZFC susceptibility of the as-grown state. However, opposite to the air-annealed state, the reduction of excess iron in the as-grown state was limited to the very surface region leaving the bulk beneath in an excess-iron-rich, weakly or non-superconducting state.

Lastly, it should be noted that the critical current density presented here is slightly lower than in some of the reported values for similar doping in the literature [Sun19]. However, as shown in section 3.4.2, the $J_{\mathrm{c}}$ presented here was extracted from MHLs that were measured at persistent fields. This leads to a decrease in the measured magnetisation due to flux creep that is less pronounced when sweeping magnetic fields quickly as is typically performed in most reports in the literature. 


\subsection{Thickness-dependent superconducting properties of $\mathrm{FeSe}_{0.35} \mathrm{Te}_{0.65}$}

The structural analysis presented in chapter 4 has shown that air annealing leads to the formation of thin iron oxide layers on the top-, bottom-, and exposed inner surfaces of $\mathrm{FeSe}_{1-x} \mathrm{Te}_{x}$. Iron is drawn from the sample to react with the oxygen and as a result the amount of excess iron in the $\mathrm{FeSe}_{1-x} \mathrm{Te}_{x}$ phase is reduced. However, the amount of excess iron is not homogeneously reduced throughout the sample. Instead, the reduction is largest in the close vicinity of the oxidised surfaces. This was shown in the SEM, STEM, and XPS analysis in the previous chapter (see sections 4.4.2, 4.5, and 4.6). Excess iron is known to be a key factor in suppressing superconductivity in this family of superconductor. The suggestion of local reduction of excess iron rather than throughout the whole sample therefore warrants the systematic investigation of the superconducting properties of different regions of an air-annealed sample.

In this section, the magnetic and superconducting properties of a stepwise exfoliated $\mathrm{FeSe}_{0.35} \mathrm{Te}_{0.65}$ single crystal are investigated for different annealing and exfoliation states. Similar to the previous sections, the as-grown sample was first characterised and then air-annealed and characterised again to probe the properties of the whole sample. After this, the sample's thickness was reduced by stepwise exfoliating the surface layers. After every exfoliation, the remaining sample's dimensions and superconducting properties were measured before the next exfoliation. In the last step, the thin exfoliated sample was annealed in air for a second time.

\subsubsection{Optical microscopy}

Figure 5.5 shows microscope images of the investigated sample at four different states from the as-grown state (a) to two different exfoliated states after air annealing (b, c), and lastly after the second air annealing of the remaining, thin, exfoliated sample. The as-grown sample was $194 \mu \mathrm{m}$ thick and shows a black, reflective surface that is 
(a)

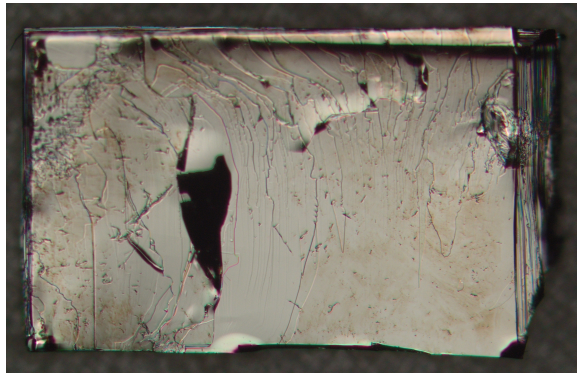

(c)

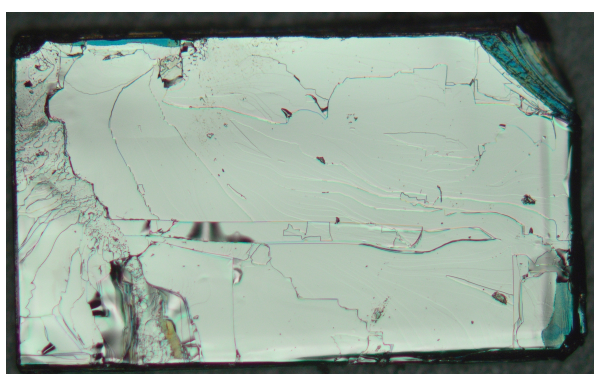

(b)

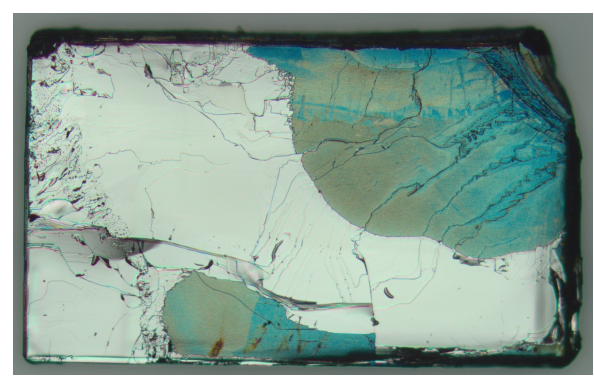

(d)

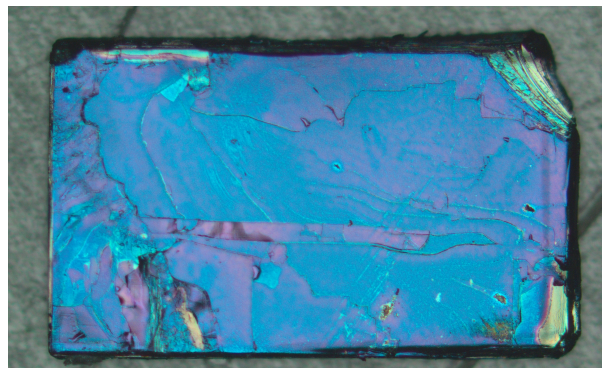

Figure 5.5: Optical microscope images of the stepwise exfoliated $\mathrm{FeSe}_{0.35} \mathrm{Te}_{0.65}$ single crystal at different exfoliation and annealing states: (a) as-grown $(197 \mu \mathrm{m})$, (b) airannealed, four times exfoliated $(113 \mu \mathrm{m})$, (c) air-annealed, five times exfoliated $(103 \mu \mathrm{m})$, (d) twice air-annealed, five times exfoliated $(103 \mu \mathrm{m})$.

typically observed for an as-grown sample (see chapter (4). After air annealing and exfoliating the surface layer four times, the thickness is reduced to $113 \mu \mathrm{m}$ and the sample surface is partially covered with an iron oxide layer (light blue layer, see section 4.3). This aligns with the observation of oxidised inner surfaces after air annealing as was shown in the previous chapter (see for instance figure 4.7). After another exfoliation step, the oxides were removed and the thickness is reduced to $103 \mu \mathrm{m}$. The last image on the bottom right shows the sample after the second annealing step. In this state, the surface is again fully covered by a blue oxide layer like after the first annealing.

Overall, the optical microscope images of the exfoliated sample reinforce that oxidation does not only take place on the top- and bottom surfaces but also in the inner parts of the sample where the surface is exposed. However, since the formation inside the bulk is determined by the gap and defect structure of the sample, it is not as uniform as the top and bottom surface. Therefore, the improvement in the superconducting properties is also likely to be less uniform in the bulk of the sample. 


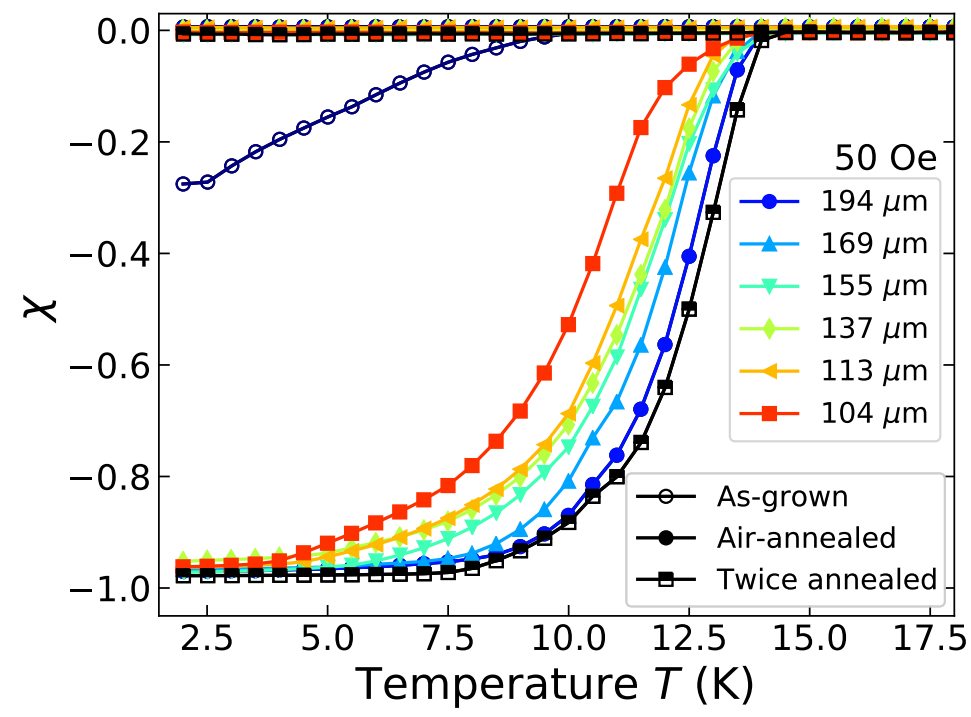

Figure 5.6: ZFC- and FC magnetisation of a $\mathrm{FeSe}_{0.35} \mathrm{Te}_{0.65}$ single crystal in the as-grown (empty circles), air-annealed (full circles), stepwise exfoliated (full symbols) and twice airannealed (top-filled square) state measured in perpendicular field at 50 Oe (@2021 IEEE).

\subsubsection{ZFC-FC susceptibility}

Figure 5.6] shows the demagnetisation corrected [Pro18] ZFC-FC susceptibility of the sample from the as-grown state, through to the twice annealed state and all exfoliation steps in-between. The as-grown sample exhibits a very broad superconducting transition, a low diamagnetic shielding fraction, and a $T_{\mathrm{c}}$ onset of $T_{\mathrm{c}}=13 \mathrm{~K}$. After air annealing, $T_{\mathrm{c}}$ is increased to $14.5 \mathrm{~K}$, the transition width is substantially reduced, and the sample exhibits perfect diamagnetic shielding $(\chi \approx-1)$. With reducing sample thickness via repeated exfoliation of the surface layers, the transition became broader as the sample got thinner. $T_{\mathrm{c}}$, however, remained unchanged and the sample still reached a full superconducting shielding fraction at $2 \mathrm{~K}$. After annealing for a second time, the transition width was significantly reduced to a width that is even less than that after the first annealing .

The observed broadening of the superconducting transition with reducing thickness is a key indicator of inhomogeneous superconducting properties. During the air annealing process, excess iron in the original near-surface region (beneath the iron oxide layer) was extracted, leading to a layer with homogeneous $T_{\mathrm{c}}$ and hence to a reduction 
in the transition width as observed in the ZFC susceptibility of the non-exfoliated, air-annealed state. However, once the top layer was removed, the remaining sample was no longer magnetically shielded by this homogeneous superconducting surface layer and the transition became broader due to an inhomogeneous $T_{\mathrm{c}}$ distribution of the sub-surface layer. Nevertheless, in comparison to the as-grown state, the transition width and $T_{\mathrm{c}}$ of the air-annealed sample is still improved at every thickness. Therefore, the excess iron concentration must, to some degree, have been reduced in the bulk of the sample as well. As shown in chapter 4 this is due to gap-like defects that allow oxygen to diffuse into the bulk of the sample and react with the iron on the exposed surfaces. This oxidation of the inner surfaces is best illustrated by the microscope image in figure 5.5(b): Even after exfoliation of the top oxide layers, the surface of the multiply exfoliated sample is partially oxidized (see blue oxide layer). However, contrary to oxidation of the top- and bottom surface, not the whole surface but only parts, where the oxygen was able to diffuse in, are oxidised. This inhomogeneous oxidation of inner parts of the sample leads to an inhomogeneous reduction of excess iron within the bulk of the sample and thus to an inhomogeneous increase of $T_{\mathrm{c}}$.

One could argue that the broadening of the superconducting transition is not the result of changes in the superconducting properties of the sample, but rather due to sample size- and geometry-dependent demagnetisation effects. In general, demagnetisation effects are expected to be more prominent and to widen the superconducting transition the thinner the sample becomes and thus could explain the observed broadening. However, as previously outlined in the experimental section 3.4.1, all ZFC-FC susceptibility measurements presented in this thesis have been demagnetisation corrected to account for these sample-geometry-dependent demagnetisation effects. Still, one could argue that the numerical demagnetisation correction corrects for macroscopic demagnetisation effects for a perfect slap with perfectly flat faces and perfect edges. In reality, this is of course not the case. For example, exfoliation of the surface layers is very likely to change the surface topography 
of the sample. Furthermore, the demagnetisation correction is based on numerical calculations with approximations, which naturally limits the accuracy [Pro18].

For this reason, to be able to unmistakably exclude geometry-dependent demagnetisation effects, the thin, exfoliated sample was air-annealed for a second time. If the change in the transition width would in fact only be driven by demagnetisation effects, annealing for a second time without changing the sample size should not severely change the transition width. However, the transition is indeed sharpened (half-filled squares in figure 5.6) and therefore the observed changes in the transition width cannot be due to demagnetisation. In fact, the sharpening of the transition width after the second air annealing reinforces the notion of homogeneous surface superconductivity beneath the oxide layer. During the second air annealing, iron oxides formed on the new surface of the sample extracting the excess iron in the region underneath. This created an excess iron-poor region with homogeneous superconducting properties as indicated by the significantly reduced transition width, that is now even smaller than that after the first annealing.

Lastly, it is worth noting that the critical temperature $\left(T_{\mathrm{c}}=13 \mathrm{~K}\right)$ is surprisingly high for an as-grown sample compared to other samples of the same $\mathrm{FeSe}_{0.35} \mathrm{Te}_{0.65}$ batch (see figure 6.3). This is likely to be an aging effect and the result of slow oxidation of the sample with time. The samples presented in figure 6.3 were measured shortly after the synthesis of the ingot and therefore are barely oxidised. The here presented sample, however, has been stored in an argon filled glovebox for two years. Even though the oxygen content is less than $0.2 \%$, the surface still slowly oxidised and the excess iron underneath was slightly reduced. This led to a higher $T_{\mathrm{c}}$ onset compared to the rest of the batch. A similar aging effect has previously been observed in electrical resistivity measurements of $\mathrm{FeTe}_{0.8} \mathrm{~S}_{0.2}$ Deg10. At first, the as-grown sample did not reach zero-resistivity. But after one day of exposure to air, the sample reached a zero-resistivity state and $T_{\mathrm{c}}$ increased continuously with increasing exposure time. Overall, however, the aging effect seems to have little effect on the superconducting properties of the air-annealed sample that still exhibits a sharp 


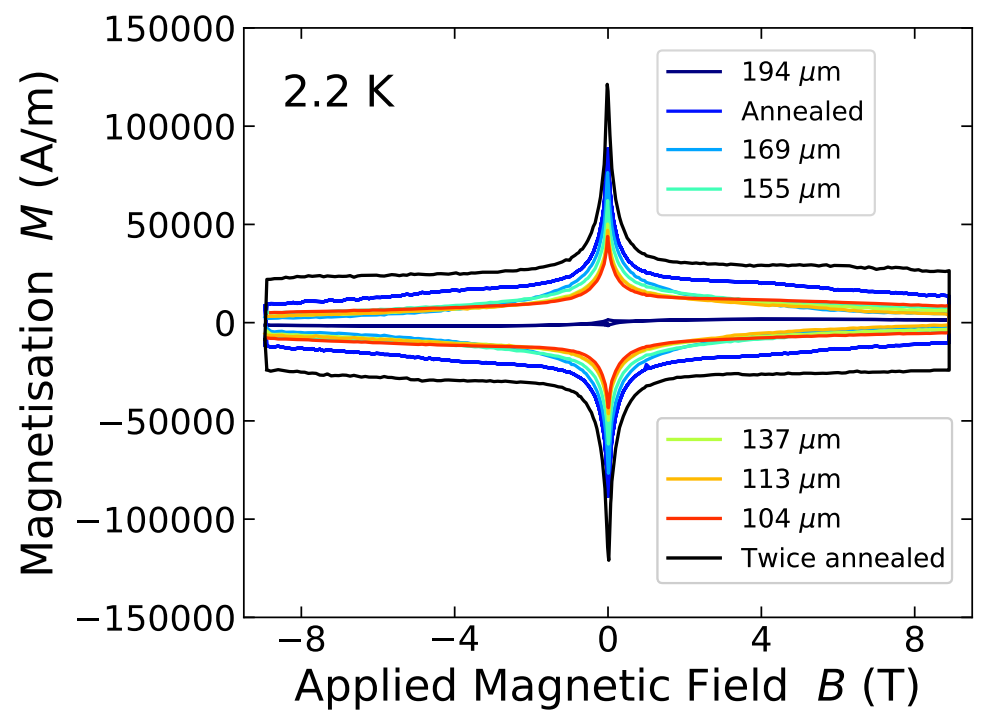

Figure 5.7: Magnetic hysteresis loops of the stepwise exfoliated $\mathrm{FeSe}_{0.35} \mathrm{Te}_{0.65}$ single crystal in the as-grown, air-annealed, and twice air-annealed state for different sample thicknesses measured at $2.2 \mathrm{~K}$. The label of the graph mark the thickness of the sample or the annealing state. For instance, '194 $\mu \mathrm{m}$ ' represents the as-grown, $194 \mu \mathrm{m}$-thick sample, 'Annealed 'the once annealed, $194 \mu \mathrm{m}$-thick sample, and 'Twice annealed 'the twice annealed, $104 \mu \mathrm{m}$-thick sample.

superconducting transition, a full superconducting shielding fraction, and an onset $T_{\mathrm{c}}$ of $14.5 \mathrm{~K}$ similar to what was observed for several air-annealed sample of this batch (see figure 6.3).

\subsubsection{Magnetic hysteresis loops}

Figure 5.7 shows the MHLs for the different annealing and exfoliation states of the sample at $2.2 \mathrm{~K}$. In analogy to the previous analysis of a sample of similar doping shown in figure 5.3(b), the as-grown sample only shows a small hysteresis loop that is greatly increased after air annealing. This of course aligns with the ZFC-FC susceptibility results presented in figure 5.6. the as-grown sample exhibits a wide superconducting transition and low diamagnetic shielding in comparison to the sharp transition and full diamagnetic shielding in the air-annealed state.

For a homogeneous superconductor, one would expect the MHLs to remain unchanged when reducing the thickness of the sample by consecutively exfoliating the 
top layer of the sample. However, the here presented MHLs demonstrate that the magnetisation at low fields continuously decreases with decreasing sample thickness. This aligns with the structural analysis in chapter 4 the reduction of excess iron is largest in the near surface region and hence the enlargement of the superconducting MHL is also largest in that region.

It is noteworthy that the change of the magnetisation at higher fields is not as monotonic as at low fields. That is, some of the thinner states exhibit a larger magnetisation at high fields than the thicker states. The high-field region of the magnetisation is highly dependent on the pinning landscape and the resulting pinning force of the sample. In fact, non-superconducting regions or regions with reduced $T_{\mathrm{c}}$, which can lead to a broadening of the superconducting transition as observed in the ZFC measurements in figure 5.6, can pin vortices and hence increase the magnetisation at high fields.

At $20 \mathrm{~K}$, the sample is not superconducting and the magnetic signal is no longer dominated by the contribution of the superconducting phase. Instead, the signal is characterised by ferromagnetic and paramagnetic contributions. Figure 5.8(a) shows the magnetisation versus applied magnetic field for all annealing- and exfoliation states at $20 \mathrm{~K}$. Overall, all sample states exhibit a typical ferromagnetic hysteresis loop with a paramagnetic background. The paramagnetic background seemingly declines with reducing sample thickness. However, this is the result of the diamagnetic background signal of the sample holder. This background is independent of sample size and therefore increasingly contributes to the total signal the smaller the sample and, thus the sample's magnetic signal, becomes. To correct for this, the magnetic moment of the empty capsule was measured and subtracted from the total magnetic moment of the actual sample measurements.

The resulting corrected magnetisation is shown in figure 5.8(b). All sample states now exhibit a relatively similar paramagnetic contribution. This clearly demonstrates that the large change in the paramagnetic signal in 5.8 (a) is not a result of annealing or exfoliation, but due to the parasitic background of the sample holder. 

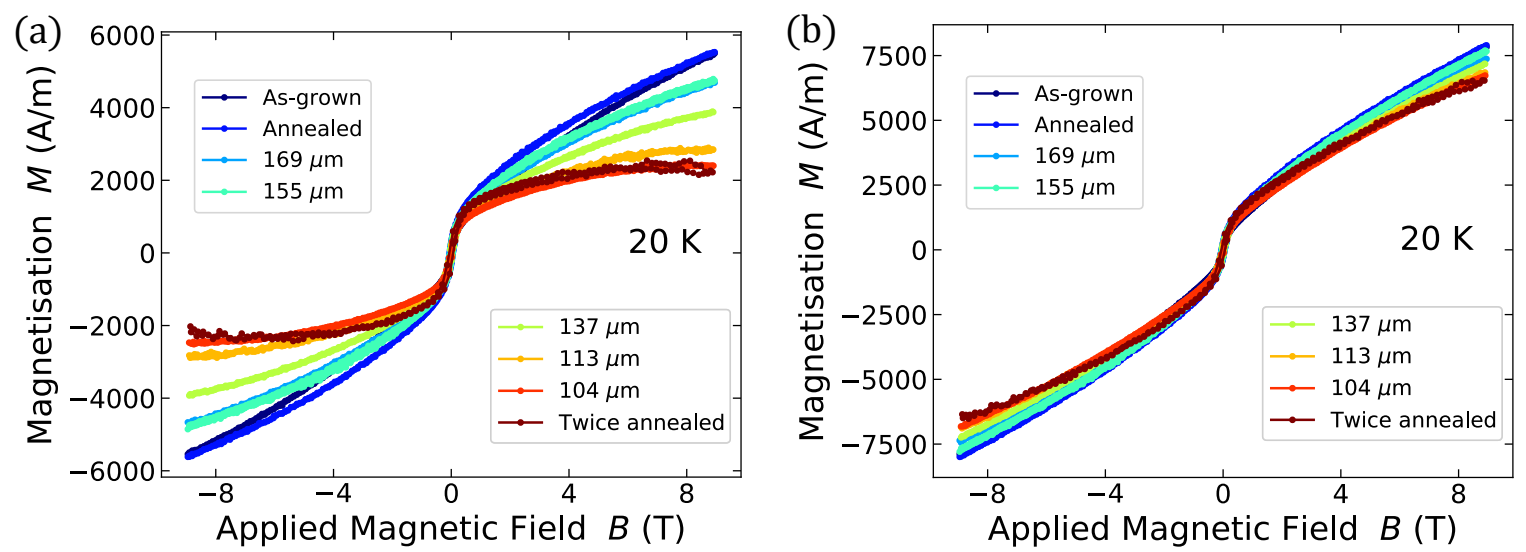

Figure 5.8: Magnetic hysteresis loops of the stepwise exfoliated $\mathrm{FeSe}_{0.35} \mathrm{Te}_{0.65}$ single crystal in the as-grown, air-annealed, and twice air-annealed state for different sample thicknesses measured at $20 \mathrm{~K}$. Figure (a) shows the measured total magnetisation and figure (b) the magnetisation after subtracting the signal of the empty sample holder.

Nevertheless, the paramagnetic signal of the sample in the different annealing and exfoliation states still varies slightly after the correction. This could however still be related to the limited accuracy of the background subtraction.

In general, the ferromagnetic signal is likely to be the result of the presence of different iron oxides. Therefore, the ferromagnetic signal is of special interest as it contains information on the oxidation level of the sample in the different annealing and exfoliation states. However, given that different iron oxides with different magnetic properties, such as hematite and magnetite, are formed, the analysis remains semi-quantitative and, as will be shown later, has in some cases to be taken with caution.

For ease of comparison and analysis of the ferromagnetic properties of the sample at the different states, the paramagnetic contribution has been estimated by fitting a linear curve to the magnetisation for fields above $6 \mathrm{~T}$ and subtracted. The remaining ferromagnetic signal after subtracting the fitted paramagnetic contribution is shown in figure 5.9(a). The as-grown sample exhibits the lowest saturation magnetisation, which increases after air annealing. Similarly, the twice annealed sample again exhibits an increased magnetisation in comparison to the different exfoliated states before the second annealing and, in fact, every state of the sample. The different 

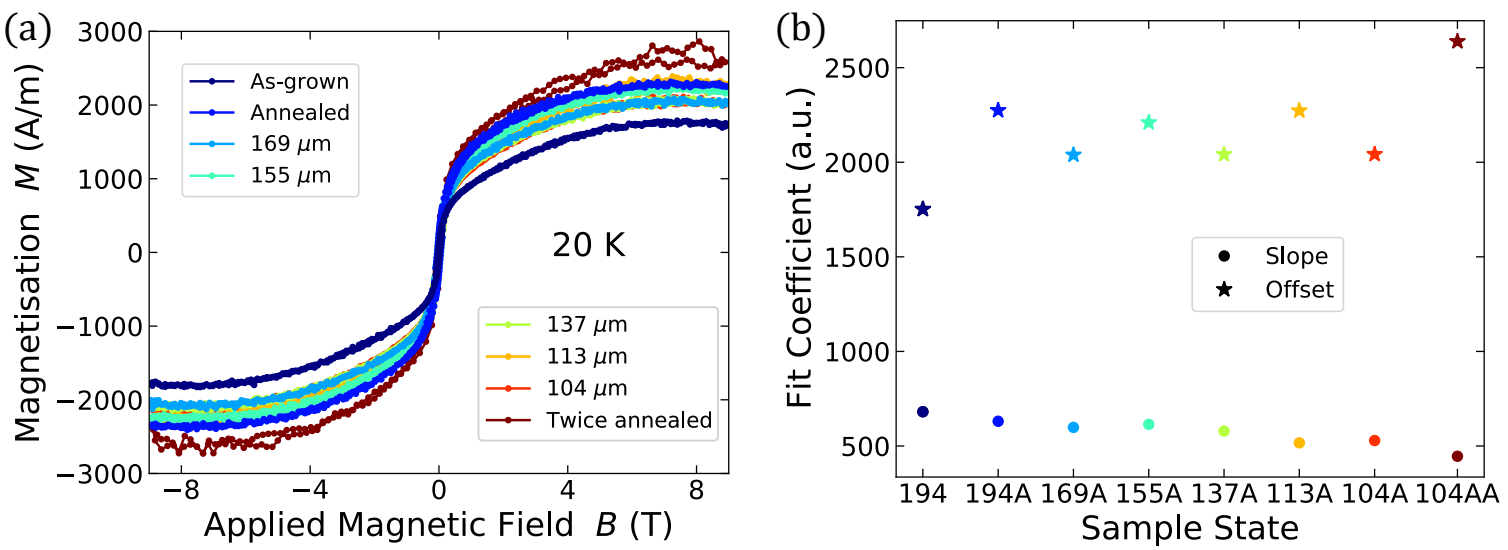

Figure 5.9: (a) Magnetic hysteresis loops of the stepwise exfoliated $\mathrm{FeSe}_{0.35} \mathrm{Te}_{0.65}$ single crystal in the as-grown, air-annealed, and twice air-annealed state after subtraction of the paramagnetic contribution for different sample thicknesses measured at $20 \mathrm{~K}$. (b) Fitting coefficients of the different sample states. The sample state is denoted as thickness in $\mu \mathrm{m}$ followed by 'A' for annealed once, and 'AA' for annealed twice.

exfoliation states, however, show no distinct trend with decreasing thickness. The change in magnetisation of the different sample states is also illustrated in figure 5.9 (b), which shows the fitted slope and offset for all samples states denoted as the thickness in $\mu \mathrm{m}$ followed by 'A' for air-annealed and 'AA' for twice air-annealed. As mentioned in the previous paragraph, the offset, which is basically the saturation magnetisation in A/m, exhibits a jump after the first air annealing (194A) and after the second air annealing (104AA). In both cases, the surface oxidised, iron oxides were formed, and thus the saturation magnetisation increased. Since the iron oxide layer is expected to be of similar thickness after the first and second annealing (see chapter 4 on the iron oxide layer thickness), the total saturation magnetisation of the twice annealed is larger due to the smaller remaining total sample volume. The saturation magnetisations of the different exfoliation states in between, however, show no distinct pattern but rather alternate between decreasing and increasing with decreasing thickness. One possible explanation for this could be the alternating removal of non-ferromagnetic and ferromagnetic layers. First, the oxidised surface layer of the air-annealed sample is removed and the magnetisation is decreased. In the next step, a non-oxidised, non-ferromagnetic layer is removed, which increases the relative amount of ferromagnetic volume and hence leads to an increase in magnetisation. 

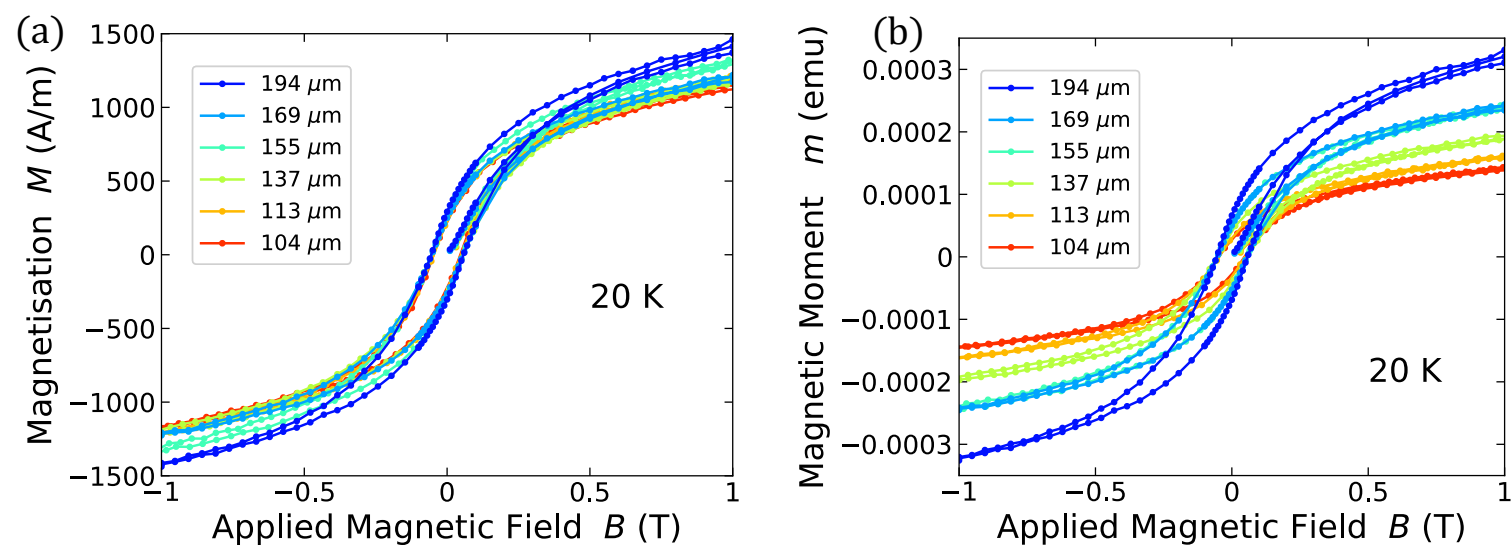

Figure 5.10: Low-field enlargement of the ferromagnetic contribution of the stepwise exfoliated $\mathrm{FeSe}_{0.35} \mathrm{Te}_{0.65}$ single crystal for all thicknesses measured at $20 \mathrm{~K}$. Figure (a) shows the magnetisation and (b) the magnetic moment.

The slope of the fit, which reflects the paramagnetic contribution, exhibits a general downward trend with decreasing thickness. This could either be due to an actual decrease in the paramagnetic signal of the sample or an artefact of the background subtraction that slightly underestimated the diamagnetic background.

Figure 5.10 (a) shows an enlargement of the MHLs of the sample at every exfoliation state for fields below $1 \mathrm{~T}$. Besides the freshly annealed $194 \mu \mathrm{m}$ thick sample state, all states exhibit almost identical hysteresis loops and even the $194 \mu \mathrm{m}$ state only slightly deviates. Given the definition of the volumetric magnetisation as $M=m / V$, where $m$ is the magnetic moment and $V$ the total volume of the sample, an almost constant value in the magnetisation implies that the magnetic moment decreases proportionally to the thickness of the sample. Therefore, the amount of iron oxide contributing to the ferromagnetic signal must also be equally distributed throughout the sample. This conclusion agrees well with the structural analysis and furthermore insinuates that the distribution of gap-like defects, which allow the oxidation of inner surfaces, are, from a macroscopic bulk perspective, relatively equally spread throughout the sample.

The change in ferromagnetic signal with thickness is further illustrated by the magnetic moment of the different exfoliation states shown in figure 5.10(b). As expected, 
the hysteresis loop of the air-annealed sample before exfoliation is largest. But even after the exfoliation of the blue iron oxide surface layer, the loop continues to shrink. This again illustrated the point made above, that the iron oxide that leads to the ferromagnetic contribution is not only on the very surface of the sample but also distributed in the bulk of the sample. There is one exception to this trend, which is the step from $169 \mu \mathrm{m}$ to $155 \mu \mathrm{m}$ at which the magnetic moment remained virtually unchanged. In general, as mentioned before, the oxidation of the inner parts of the sample depends on the distribution of gap-like defects. Given the, to some extent, uncontrollable nature of the exfoliation process and the expected random distribution of iron oxide throughout the sample, there is always a non-zero chance that a removed layer actually did not contain any ferromagnetic oxide species. This is likely to be the reason why there was no change in the magnetic moment between $169 \mu \mathrm{m}$ and $155 \mu \mathrm{m}$.

Figure 5.11(a) shows an enlargement of the MHLs before and after every annealing step for fields below $1 \mathrm{~T}$. As previously noted above, the saturation magnetisation is larger after both annealing steps in comparison to the state right before. Additionally, the magnetisation before and after the second annealing step are both larger than before and after the first annealing, respectively. This is expected and aligns with the structural analysis in the previous chapter 4. The original as-grown state contains the lowest amount of ferromagnetic oxide per volume. After the first air annealing, an iron oxide surface layer has formed on the top- and bottom surface and any inner surface exposed to air during annealing. As a result, the ferromagnetic signal is increased. When the top- and bottom surface layers were removed and the thickness was reduced from $194 \mu \mathrm{m}$ to $104 \mu \mathrm{m}$, the magnetisation decreased. Again, this is sensible, given that the oxidation of the inner regions of the sample is subject to random gap-defects, the oxide density is expected to be largest on the top- and bottom surface, where the top $100 \mathrm{~nm}$ consist purely of iron oxide (see chapter 4). Nevertheless, the magnetisation of the 104A state is still larger than in the as-grown state because of the ferromagnetic oxides that have formed on the exposed inner 

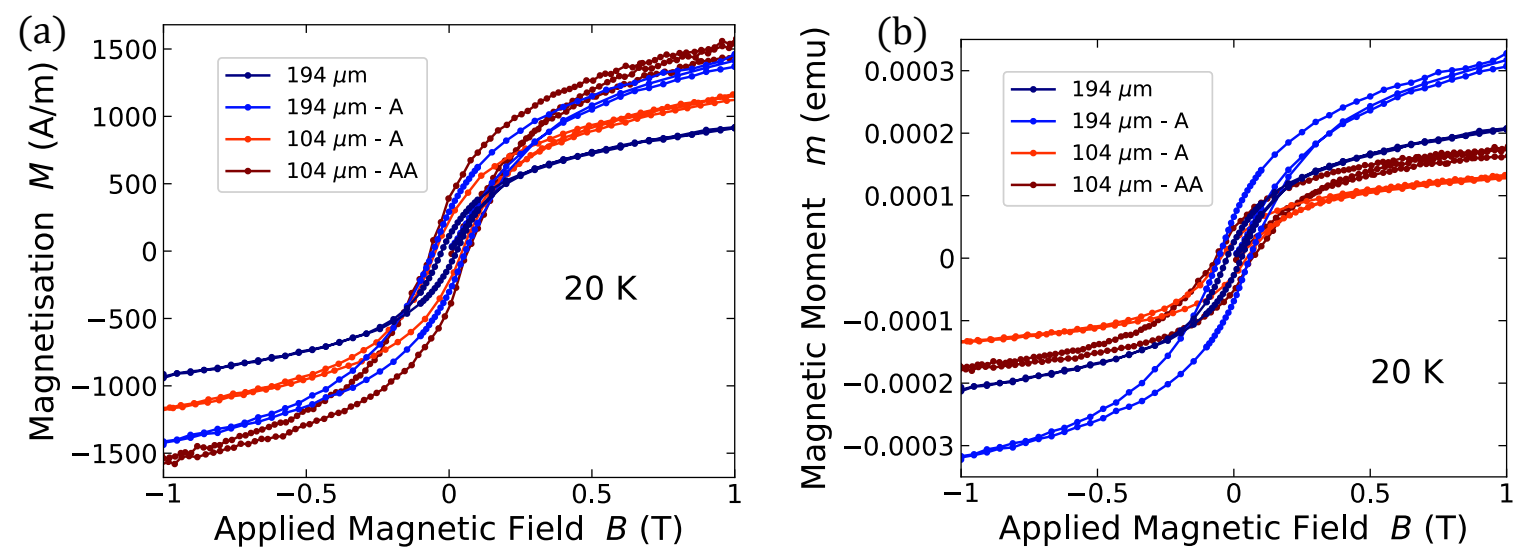

Figure 5.11: Low-field enlargement of the ferromagnetic contribution of the stepwise exfoliated $\mathrm{FeSe}_{0.35} \mathrm{Te}_{0.65}$ single crystal before and after the two annealing states measured at $20 \mathrm{~K}$. Figure (a) shows the magnetisation and (b) the magnetic moment.

surfaces of the sample.

Analogous to after the first annealing, a continuous iron oxide surface layer formed after the second annealing, thus, leading to an increase in the magnetisation. At this point, the total sample thickness is reduced almost by half and thus the top and bottom oxide layer now contribute a larger portion to the total signal. Additionally, some inner surfaces might have continued to oxidise or new gap-defects might have formed to further increase the iron oxide density. As a result of the two, the magnetisation of the twice annealed state is larger than the once annealed state.

So far, the analysis has been focused on the changes in magnetisation in different exfoliation or annealing states. However, when looking at the absolute values, it is most notable that even the as-grown sample exhibits a large ferromagnetic signal. In fact, the saturation magnetisation after both the first and second annealing only increased by about $20 \%$ compared to the state before. Therefore, the as-grown sample must already have contained a considerable amount of magnetic species. As stated before, the batch from which this specific sample was extracted has been synthesised two years before the actual characterisation. It is thus not surprising that the exposed surfaces of the as-grown sample have already started to oxidise before air annealing. This oxidation must have led to the removal of excess iron from 
the sample and hence should have induced an improvement in the superconducting properties. However, the observed improvement is quite minute in comparison to the air annealed state. Based on the Raman analysis in chapter 4 and the reaction conditions during annealing [Wri91], magnetite is believed to be the origin of the ferromagnetic signal. Therefore, using the saturation magnetisation of bulk magnetite, $M_{\text {sat,mag }}=446 \mathrm{kA} / \mathrm{m}[$ Kem16], the relative volume of the magnetite in the sample as a fraction of the total sample volume can be estimated as follows

$$
\frac{V_{\text {sample,mag }}}{V_{\text {sample }}}=\frac{M_{\text {sat,sample }}}{M_{\text {sat,mag }}}
$$

where $V_{\text {sample,mag }}$ is the volume of the magnetite in the sample, $V_{\text {sample }}$ is the total sample volume, and $M_{\text {sat,sample }}$ is the saturation magnetisation of the sample. From this, only approximately $0.4 \%$ of the total volume are expected to be magnetite. The iron content of this amount of magnetite is less than $1 \%$ of the total iron content of the sample (see table 4.4). Therefore, it is not too surprising that the superconducting properties have not been significantly improved as the typical excess iron concentration in this compound has been estimated to approximately $8 \%$ for this batch via SEM and is reported in the literature to be up to 14\% [Sun19]. Overall, this finding insinuates that the formation of magnetite due to exposure to oxygen at room temperature does not remove enough excess iron to significantly improve the superconducting properties. Higher temperatures are required to promote the formation of hematite [Wri91] and to allow iron from deeper inside the sample to diffuse towards an exposed surface. Both processes are vital for the removal of larger amount of excess iron and the resulting improvement the superconducting properties. In general, the improvement in the superconducting properties cannot be directly be inferred from the absolute value of the ferromagnetic signal of a sample. The change in the ferromagnetic signal from before to after annealing, however, can be indicative of increased oxidation and a resulting change in the superconducting properties. This is illustrated in figure 5.11(b), which shows the magnetic moment of the sample before 


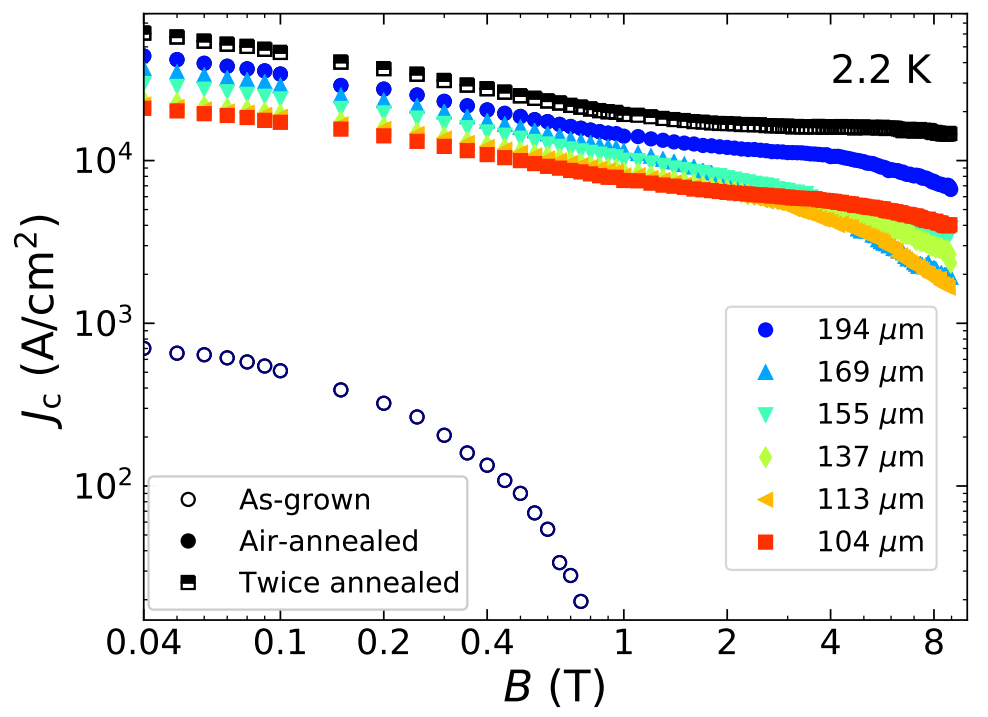

Figure 5.12: $J_{\mathrm{c}}$ versus $B$ of the stepwise exfoliated $\mathrm{FeSe}_{0.35} \mathrm{Te}_{0.65}$ single crystal in the asgrown, air-annealed, and twice air-annealed state for different sample thicknesses measured at $2.2 \mathrm{~K}$ (@2021 IEEE).

and after every annealing step for fields below $1 \mathrm{~T}$. The magnetic moment of the asgrown sample at $1 \mathrm{~T}$ is larger than both the once and twice annealed state $104 \mu \mathrm{m}$ thick state. However, the superconducting properties of the latter two are largely improved from the as-grown state.

\subsubsection{Critical Current Density}

Lastly, using Bean's critical state model [Bea62, Bea64, $J_{\mathrm{c}}$ at $2.2 \mathrm{~K}$ was extracted from the magnetic hysteresis loop measurements as presented for the different states of the sample in figure 5.12 . Overall, the critical current density exhibits a similar behaviour as the critical temperature. $J_{\mathrm{c}}$ is very low for the as-grown sample and is increased after the first air annealing. For fields below $1 \mathrm{~T}$, the exfoliation of the surface layers and reduction of the thickness of the sample led to a monotonic decrease in $J_{\mathrm{c}}$. At higher fields, $J_{\mathrm{c}}$ does not follow the same trend, which is likely due to a variation in the pinning landscape. Contrary to $T_{\mathrm{c}}, J_{\mathrm{c}}$ might actually benefit from inhomogeneity in the sample that can act as flux pinning centres at high fields. This effect is especially pronounced for the $104 \mu \mathrm{m}$ state for which $J_{\mathrm{c}}$ decreases less rapidly 
at high fields. Nevertheless, after the second annealing, the sample's $J_{\mathrm{c}}$ is again increased over the whole measured magnetic field range. Since the sample is now thinner than after the first annealing, the superconducting layer underneath the oxide contributes a larger portion to the overall signal leading to a larger critical current density than after the once annealed, thick sample. Both the ZFC-FC susceptibility and $J_{\mathrm{c}}$ therefore support the notion that the largest reduction of excess iron near the surface also leads to the largest improvement in the superconducting properties in the near-surface region.

\subsection{Conclusion}

In conclusion, air annealing has been shown to improve the superconducting properties of $\mathrm{FeSe}_{1-x} \mathrm{Te}_{x}$ for the four investigated selenium concentrations: 0.15, 0.35, 0.5, and 0.65. The $\mathrm{FeSe}_{0.15} \mathrm{Te}_{0.85}$ sample was non-superconducting in the as-grown state and became superconducting with a critical temperature of $12.8 \mathrm{~K}$ after air annealing. The critical temperature of the optimally doped sample, $\mathrm{FeSe}_{0.5} \mathrm{Te}_{0.5}$, was slightly reduced after annealing, but the critical current density improved by approximately $40 \%$ for fields up to $9 \mathrm{~T}$. The slightly under- and over doped samples both exhibited an increase in $T_{\mathrm{c}}$, sharpening of the superconducting transition, and an increase in $J_{\mathrm{c}}$ by more than one order of magnitude.

The analysis of a stepwise exfoliated sample showed that the improvement in superconducting properties, such as the superconducting transition width and $J_{\mathrm{c}}$, is largest in the near-surface region just beneath the iron oxide layer. This finding aligns with the earlier finding in the structural chapter 4 that showed the reduction in excess iron to be largest in the near surface region beneath the iron oxide. Furthermore, this also reinforces the reduction of excess iron as the driving factor in the improvement of the superconducting properties after annealing.

Overall, the results of this chapter show that post-processing of the as-grown single crystals by methods such as air-annealing is vital for the $\mathrm{FeSe}_{1-x} \mathrm{Te}_{x}$ family of su- 
perconductors. Furthermore, the analysis of the stepwise exfoliated sample and the finding of largest improvement in the near surface region illustrate that optimizing the annealing conditions to obtain greater $J_{\mathrm{c}}$ might also be achieved by considering the geometry and thickness of a sample and, for example, maximising the surface to volume ratio. 



\section{Effect of annealing in inert atmospheres on $\mathbf{F e S e}_{1-x} \mathbf{T e}_{x}$}

In the previous chapter, it was shown that annealing $\mathrm{FeSe}_{1-x} \mathrm{Te}_{x}$ single crystals in air improves both their critical temperature as well as their critical current density. This improvement was linked to the reduction of the excess iron concentration within the sample driven by the reaction of oxygen with iron during annealing. Similarly, annealing in various other atmospheres, such as Te [Kos13, Sun13a], Se [Sun13b], I [Rod11], S [Sun13b], and As [Zho14], have also been reported to lead to the formation of a thin iron-compound surface layer and a resulting improvement in the superconducting properties. However, the beneficial effect of annealing has also been reported for annealing in inert atmospheres such as nitrogen $[\mathrm{Hu} 12 \mathrm{a}$ ] and vacuum [Noj10. This is surprising, given that neither nitrogen [Ert79, Met87] nor vacuum annealing should lead to the formation of iron-compounds under typical annealing conditions. Therefore, the question arises if the observed improvements in the superconducting properties in these scenarios are also driven by a reduction in the amount of interstitial excess iron and, if so, what process would be driving it.

In this chapter, the effect of annealing in inert atmospheres (nitrogen, low- and high vacuum) is investigated with a focus on the surface chemistry and the resulting changes in the superconducting properties of $\mathrm{FeSe}_{0.35} \mathrm{Te}_{0.65}$. First, the emergence of a universal superconducting phase diagram for annealed $\mathrm{FeSe}_{1-x} \mathrm{Te}_{x}$ from this work and the literature is uncovered and discussed. Next, a comprehensive study of the surface chemistry and the superconducting properties of in nitrogen, air, highand low-vacuum annealed $\mathrm{FeSe}_{0.35} \mathrm{Te}_{0.65}$ single crystals is presented. Specifically, all samples were characterised using Raman spectroscopy, ZFC-FC susceptibility, and MHL measurements. Lastly, the amount of residual oxygen present during annealing is estimated using the ideal gas law. 

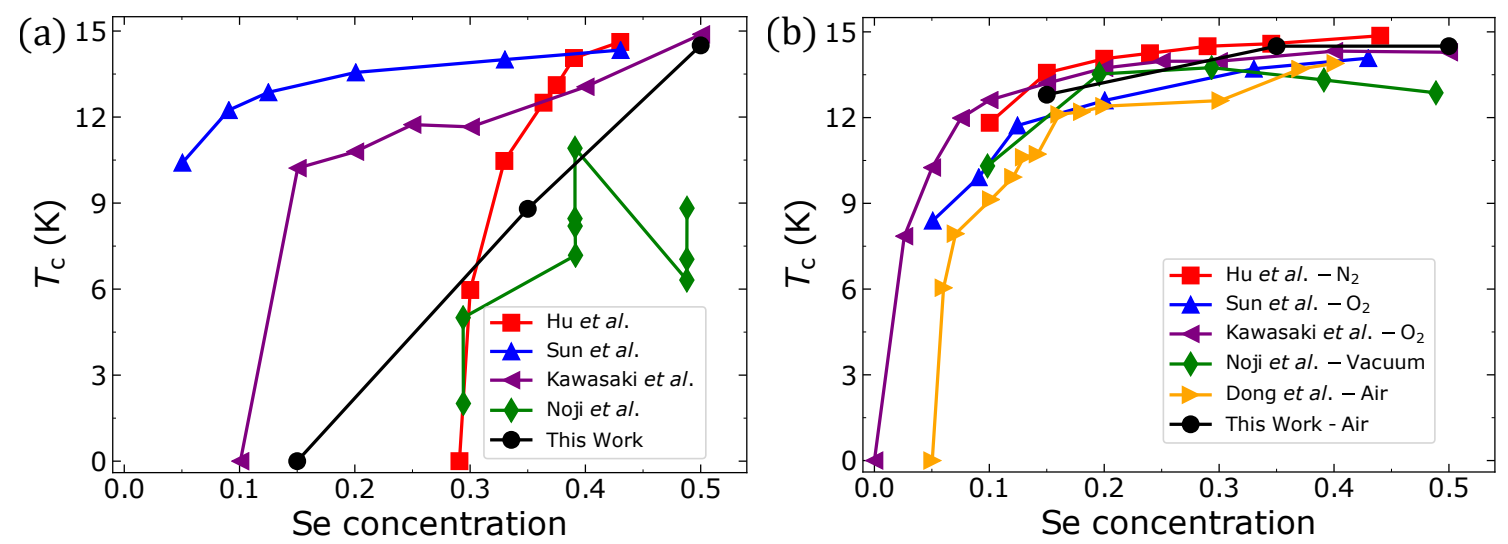

Figure 6.1: (a) Plot of $T_{\mathrm{c}}$ versus the Se concentration for as-grown $\mathrm{FeSe}_{1-x} \mathrm{Te}_{x}$ single crystals from the literature [Hu12a, Sun16, Kaw12, Noj10] and from this work (filled circles). (b) Plot of $T_{\mathrm{c}}$ versus the Se concentration for $\mathrm{FeSe}_{1-x} \mathrm{Te}_{x}$ single crystals from the same studies as in (a) [Hu12a, Sun16, Kaw12, Noj10, one additional study [Don11], and from this work (filled circles) after annealing in different atmospheres.

\subsection{Emergence of a universal superconducting phase dia- gram}

Figure 6.1(a) shows the critical temperature of as-grown $\mathrm{FeSe}_{1-x} \mathrm{Te}_{x}$ single crystals from several reports [Hu12a, Sun16, Kaw12, Noj10] and this work for different selenium concentrations. The critical temperatures of the as-grown samples with similar Se concentration are found to vary greatly between different reports. In fact, not only is there a wide variation in $T_{\mathrm{c}}$ between different reports, but as in the case of Noji et al. Noj10, even individual studies report a wide range of critical temperatures for samples with the same selenium concentration. Overall, $T_{\mathrm{c}}$ increases with increasing Se concentration reaching a maximum value around $14.5 \mathrm{~K}$ at optimum doping with a composition of $\mathrm{FeSe}_{0.5} \mathrm{Te}_{0.5}$. Figure 6.1(b) shows the corresponding $T_{\mathrm{c}}$ of the same reports [Hu12a, Sun16, Kaw12, Noj10, one additional report [Don11, and this work after the samples were annealed in various atmospheres or under vacuum. Besides the general increase in $T_{\mathrm{c}}$ after annealing, the figure also reveals that annealing in various atmospheres leads to a universal superconducting phase diagram that is seemingly independent of the initial properties of the as-grown samples. 
The absence of a universal superconducting phase diagram for the as-grown $\mathrm{FeSe}_{1-x} \mathrm{Te}_{x}$ samples is likely to be the result of different interstitial excess iron concentrations in the non-annealed state that arise from variations in the synthesis conditions. As previously mentioned, excess iron is known to suppress $T_{\mathrm{c}}$ McQ09, Zha09, Lei10, Liu09, Don11, and hence variations in the excess iron fraction lead to differences in $T_{\mathrm{c}}$. After annealing, the excess iron fraction is reduced to a common value, which leads to the emergence of the universal superconducting phase diagram for all six studies. This vividly illustrates that annealing is crucial to obtain reproducible high-quality samples as was also previously pointed out in other studies [Hu12b, Yam16, Lim18, Sun19].

Even though the beneficial effect of annealing has been widely studied [Hu12a, Sun16, Kaw12, Noj10, Don11, Sun19, the underlying physics of the process that leads to the observed improvement in the superconducting properties is still not fully understood. Specifically, figure 6.1(a) might suggest that annealing in different atmospheres has a similar effect on $T_{\mathrm{c}}$, but there is substantial disagreement in the literature over the beneficial effects of annealing in different atmospheres [Sun16, Kaw12]. For example, annealing in inert atmospheres such as vacuum or nitrogen have both been found to have a beneficial effect [Hu12a, Noj10, as well as no effect at all Sun13. To resolve this dispute in the literature, a series of experiments has been designed to systematically investigate the effect of annealing in air, nitrogen, low- and highvacuum on the surface chemistry using Raman spectroscopy. The latter technique has rarely been deployed in this field, but is highly suited for the investigation of the surface chemistry of annealed $\mathrm{FeSe}_{1-x} \mathrm{Te}_{x}$ single crystals especially because of the formation of iron oxide species (see chapter 4). The insights on the changes in the surface chemistry from the Raman analysis are then linked to changes in the superconducting properties such as the critical temperature, the superconducting transition width, and the critical current density. 


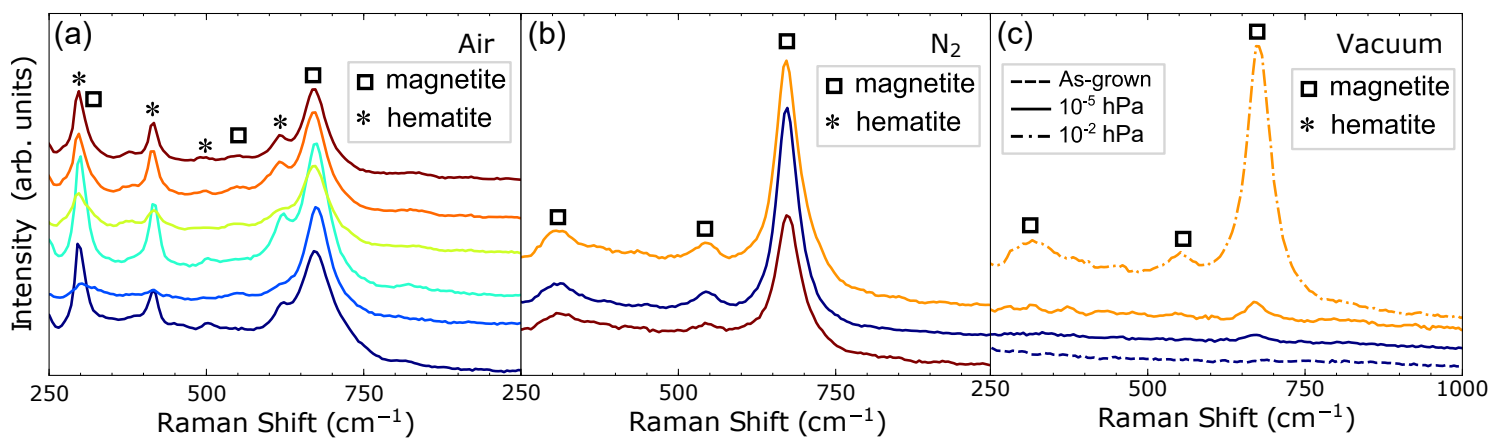

Figure 6.2: Raman spectra of several $\mathrm{FeSe}_{0.35} \mathrm{Te}_{0.65}$ samples after annealing in air (a), nitrogen (b), and under vacuum (c). For ease of comparison, the spectra of the different samples have been shifted on the vertical axis.

\subsection{Raman analysis of as-grown and annealed $\mathrm{FeSe}_{0.35} \mathrm{Te}_{0.65}$}

Figure 6.2 shows Raman spectra of several $\mathrm{FeSe}_{0.35} \mathrm{Te}_{0.65}$ single crystals in the asgrown state and after annealing in air [Boc1], nitrogen (oxygen $<5$ ppm ) Boc2] , low- $\left(p \approx 10^{-2} \mathrm{hPa}\right)$ and high vacuum conditions $\left(p<10^{-5} \mathrm{hPa}\right)$. As demonstrated in chapter 4, air annealing leads to the formation of magnetite $\left(\mathrm{Fe}_{3} \mathrm{O}_{4}\right)$ and hematite $\left(\alpha-\mathrm{Fe}_{2} \mathrm{O}_{3}\right)$ on the surface of the sample. This is can again be inferred from the Raman peaks exhibited by the six air-annealed samples show in figure 6.2(a).

The Raman spectra of three nitrogen annealed samples are shown in figure 6.2(b). Interestingly, iron oxide is again evident in the Raman spectra, but in this case, only magnetite, which has a lower iron oxidation state $\left(\mathrm{Fe}^{2+}\right.$ and $\left.\mathrm{Fe}^{3+}\right)$ compared to hematite (strictly $\mathrm{Fe}^{3+}$ ), was detected. Still, the appearance of any iron oxide on the surface of the samples is surprising. It suggests that even a low oxygen fraction (oxygen $<5 \mathrm{ppm}$ ) is enough to induce the formation of significant amounts of iron oxide during annealing.

The Raman spectra of low- and high-vacuum annealed samples are shown in figure 6.2(c). In the case of annealing under high-vacuum conditions, the Raman spectra are almost unchanged after annealing except for a minute peak from the magnetite Raman mode at $667 \mathrm{~cm}^{-1}$. In fact, one of the samples (figure 6.2(c), orange curve) was annealed for 24 hours to demonstrate that even prolonged annealing under high 
vacuum does not lead to the formation of substantial amounts of iron oxide. After the 24 hours of high-vacuum annealing and subsequent characterisation, the same sample was annealed under low vacuum conditions for 2 hours. In contrast to high vacuum annealing, two hours of low vacuum annealing sufficed to initiate the formation of an iron oxide surface layer of magnetite, similar to that observed for nitrogen annealing. Overall, the Raman analysis insinuates that there must still be a significant amount of residual oxygen present in the low vacuum and nitrogen annealing atmosphere to facilitate the formation of iron oxide. It is only under high-vacuum conditions that the amount of residual oxygen is reduced enough to prevent the formation of an iron oxide surface layer during annealing.

\subsection{ZFC-FC susceptibility of as-grown and annealed $\mathrm{FeSe}_{0.35} \mathrm{Te}_{0.65}$}

Figure 6.3 shows the ZFC-FC magnetic susceptibility of the same as-grown (dashed lines) and annealed (full lines) samples as presented in the Raman analysis in figure 6.2. For ease of comparison, the susceptibility and Raman data of each sample is represented using the same colour within the respective annealing conditions. Each susceptibility curve was corrected to account for demagnetisation effects following Prozorov et al. as described in detail in experimental section 3.4.1 [Pro18].

The as-grown samples show a significant variance in $T_{\mathrm{c}}$, the superconducting transition width, and only partial diamagnetic shielding at the lowest temperature. This again illustrates that, analogous to the report of Noji et al. Noj10, even samples from the same batch can exhibit a large variance in the superconducting properties. After annealing in air (see figure 6.3 (a)), the critical temperature, superconducting transition width, and diamagnetic shielding fraction are largely increased for all samples in analogy to what was presented in the previous chapter 5. Furthermore, all samples exhibit similar superconducting transitions with $\chi(2 \mathrm{~K}) \approx-1$ and $T_{\mathrm{c}} \approx 14 \mathrm{~K}$. 


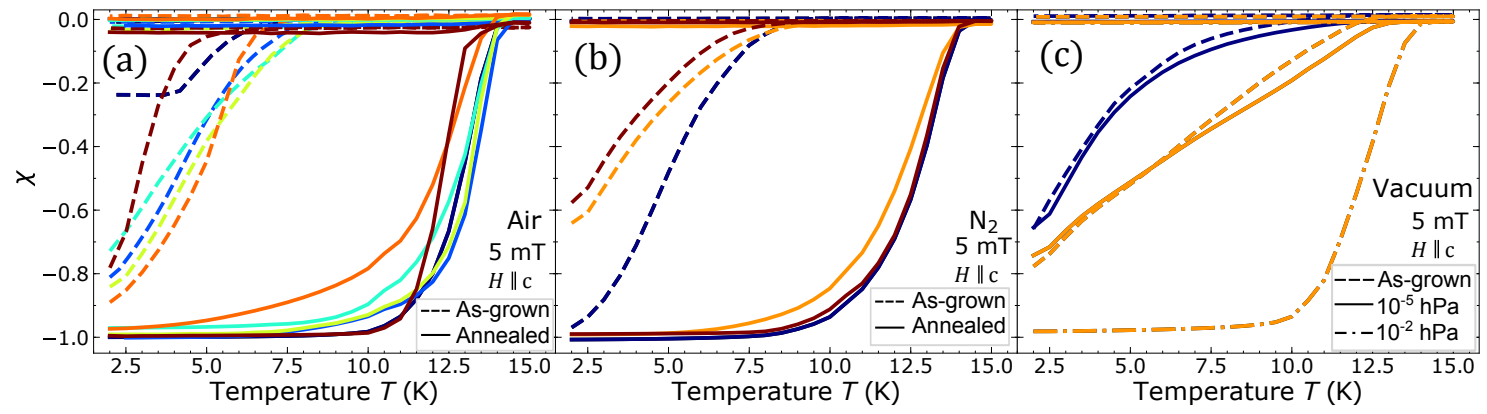

Figure 6.3: Plot of the demagnetisation-corrected ZFC-FC magnetic susceptibility of several $\mathrm{FeSe}_{0.35} \mathrm{Te}_{0.65}$ samples after annealing in air (a), nitrogen (b), and under vacuum (c). For ease of comparison, the data for each sample is represented by the same colour as in figure 6.2 for each respective annealing condition.

Interestingly, nitrogen annealing (see figure 6.3(b)) also led to an increase in $T_{\mathrm{c}}$, a reduction in the transition width, and perfect diamagnetic shielding, very similar to what is observed in the air-annealed samples. Additionally, the nitrogen-annealed samples also exhibit almost identical ZFC curves independent of the variance in all three properties in the as-grown state before annealing.

On the contrary, high-vacuum annealing (figure 6.3(c)) has little effect on $T_{\mathrm{c}}$, the temperature dependence of the ZFC susceptibility, and the diamagnetic shielding at the lowest temperature. However, annealing the same sample again under lowvacuum conditions increased $T_{\mathrm{c}}$, the diamagnetic shielding at $2 \mathrm{~K}$, and reduced the transition width similarly to nitrogen annealing.

\subsection{Critical current density of as-grown and annealed $\mathrm{FeSe}_{0.35} \mathrm{Te}_{0.65}$}

The effect of annealing in different atmospheres on $J_{\mathrm{c}}$ for the same samples as in the Raman spectroscopy and ZFC-FC susceptibility measurement above is presented in figure 6.4. Analogous to $T_{\mathrm{c}}, J_{\mathrm{c}}$ of the as-grown samples (dashed lines) is relatively low and also varies largely between $3 \times 10^{3} \mathrm{~A} / \mathrm{cm}^{2}$ and $2 \times 10^{4} \mathrm{~A} / \mathrm{cm}^{2}$ for the different samples. After annealing in air, the critical current density of all samples is increased 


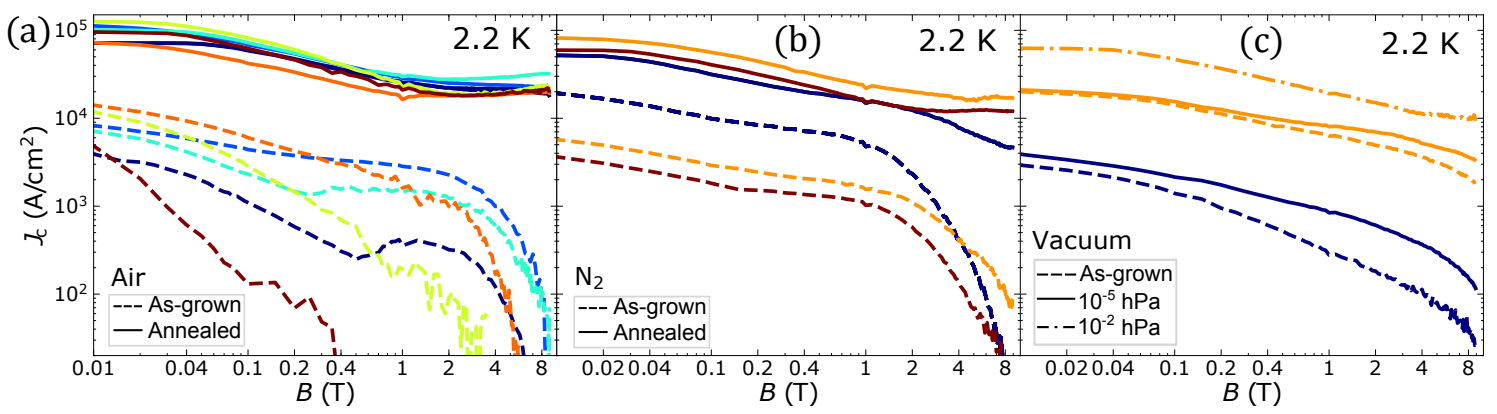

Figure 6.4: Plot of $J_{\mathrm{c}}$ of several $\mathrm{FeSe}_{0.35} \mathrm{Te}_{0.65}$ samples after annealing in air (left), nitrogen (center), and under vacuum (right). For ease of comparison, the data for each sample is represented by the same colour as in figure 6.2 and 6.3 for each respective annealing condition.

by one order of magnitude independent of the as-grown $J_{\mathrm{c}}$ similar to what was observed in chapter 5. Furthermore, the variance in $J_{\mathrm{c}}$ between the different samples in the as-grown state was also largely reduced. Annealing in nitrogen and low vacuum also resulted in an increase in $J_{\mathrm{c}}$. However, the increase is slightly smaller with $J_{\mathrm{c}}$ not exceeding $10^{5} \mathrm{~A} / \mathrm{cm}^{2}$ for all samples compared to the air-annealed samples that reach critical currents above this threshold. In contrast to all other annealing atmospheres, high vacuum annealing induced almost no change in the low-field $J_{\mathrm{c}}$ below $0.1 \mathrm{~T}$ even after 24 hours of annealing. There is a slight increase in $J_{\mathrm{c}}$ for higher fields, but it is still more than an order of magnitude lower than the $J_{\mathrm{c}}$ of air annealed samples.

\subsection{Summary}

Overall, the results from the Raman spectroscopy and the magnetic measurements present a clear link between the improvement in the superconducting properties and the formation of iron oxide on the surface: Air, nitrogen, and low vacuum annealing induced the formation of iron oxide and improved the superconducting properties whereas high vacuum annealing, after which only a very small amount of iron oxide was formed, had little effect on the superconducting properties. These results therefore imply that increasing homogeneity by annealing at high temperatures alone cannot be the driving factor for the improvement in the superconducting properties. 
Instead, the notion of interstitial excess iron reduction via the formation of iron oxides as the driving factor for the improvement in the superconducting properties is being reinforced.

Interestingly, the improvement in the superconducting properties after nitrogen and low vacuum annealing is smaller in comparison to air annealing. This correlates with the Raman analysis showing that hematite, with Fe in a higher oxidation state in comparison to magnetite, did not form after low vacuum or nitrogen annealing, but only after air annealing. Given that the sealed nitrogen, and low vacuum annealing atmosphere should only contain a limited amount of residual oxygen in comparison to air annealing with continuous air flow, it is intuitive that air annealing should lead to a higher degree of oxidation [Wri91]. As a result, more iron should be removed from the sample in the case of air annealing in comparison to nitrogen and low vacuum annealing, which led to a smaller improvement in the superconducting properties for the latter two.

\subsection{Estimation of residual oxygen present in different an- nealing atmospheres}

Based on the analysis above, the formation of iron oxide on the surface of the single crystals is the driving factor for the improvement in the superconducting properties of air, nitrogen, and low-vacuum annealed $\mathrm{FeSe}_{1-x} \mathrm{Te}_{x}$. This oxidation of the surface is expected for oxygen containing atmospheres like air, but surprising for low vacuum and nitrogen annealing. In this section, the notion that residual oxygen is inherently present in nitrogen and low vacuum atmospheres is reinforced by estimating the amount of residual oxygen present during annealing for the experimental setup used above. The following estimation of residual oxygen is based on two assumptions:

(1) The respective atmosphere can be described by the ideal gas law. 
(2) The number of atoms in the annealing chamber is conserved.

In our analysis, the two atmospheres of interest are nitrogen and air at reduced pressure (low-vacuum and high-vacuum) and room temperature. Under these conditions, both, nitrogen and air are well described by the ideal gas law [Bro99]. The second assumption concerns the particle conservation of the annealing atmosphere. Essentially, an ideal experimental setup that allows no air to diffuse into or out of the chamber during annealing is assumed. In reality, this is strictly speaking impossible. However, given the high vacuum conditions that were obtained with the setup used in this thesis, this assumption is a good approximation.

The ideal gas law is given by

$$
p V=N k_{\mathrm{B}} T
$$

where $k_{\mathrm{B}}$ is the Boltzmann constant, $p$ is the pressure, $V$ the volume, $T$ the temperature, and $N$ the number of atoms. For the case of the annealing atmosphere here, the relevant parameters are the temperature at which the furnace was filled with the annealing gas or pumped to low- or high vacuum, $T=T_{\text {room }}$, and the volume of the furnace, $V=V_{\text {fur }}$. The calculations were performed for the three annealing atmospheres used in the experiments presented above: nitrogen $(99.998 \%$ pure nitrogen with oxygen $<5 \mathrm{ppm})$, low vacuum $\left(p_{\text {vac,low }}=10^{-2} \mathrm{hPa}\right)$, and high vacuum $\left(p_{\text {vac,high }}=10^{-25} \mathrm{hPa}\right)$. All parameters for the calculation are summarised in table 6.1 .

Table 6.1: Parameters from the annealing setup used to estimate the residual oxygen in each atmosphere.

\begin{tabular}{r|c|c|c|c} 
Furnace Volume & $T_{\text {room }}$ & Low vacuum & High vacuum & Nitrogen pressure \\
\hline 0.741 & $298 \mathrm{~K}$ & $10^{-2} \mathrm{hPa}$ & $10^{-5} \mathrm{hPa}$ & $1013 \mathrm{hPa}$
\end{tabular}

The total number of atoms $N_{\text {atom }}$ in the annealing atmosphere can be calculated by 
rewriting the ideal gas law as such

$$
N_{\text {atom }}=\frac{k_{\mathrm{B}} T}{p V}
$$

Based on the total number of atoms $N_{\text {atom }}$ in the respective atmosphere, the amount of residual oxygen can be estimated by multiplying with the oxygen concentration of the atmosphere. In the case of vacuum, the relevant oxygen concentration is that of the residual air with an oxygen concentration of $21 \%$ Boc1. For nitrogen, an upper estimate of $5 \mathrm{ppm}$ is given by the manufacturer [Boc2]. The total number of atoms $N_{\text {atom }}$, the respective oxygen concentration, and the number of oxygen atoms $N_{\text {oxygen }}$ present in the respective atmosphere are summarised in table 6.2 .

Table 6.2: Total number of atoms $N_{\text {atom, }}$ oxygen concentration, and number of residual oxygen atoms $N_{\text {oxy }}$ for nitrogen, low vacuum, and high vacuum annealing.

\begin{tabular}{r|c|c|c} 
Atmosphere & $N_{\text {atom }}$ & Oxygen Concentration & $N_{\text {oxy }}$ \\
\hline Nitrogen $\left(10^{3} \mathrm{hPa}\right)$ & $1.8 \times 10^{22}$ & $0.005 \%$ & $9.1 \times 10^{16}$ \\
Low-vacuum $\left(10^{-2} \mathrm{hPa}\right)$ & $1.8 \times 10^{17}$ & $21 \%$ & $3.8 \times 10^{16}$ \\
High-vacuum $\left(10^{-5} \mathrm{hPa}\right)$ & $1.8 \times 10^{14}$ & $21 \%$ & $3.8 \times 10^{13}$
\end{tabular}

It can immediately be seen that the residual oxygen present in the low vacuum and nitrogen atmosphere are on the same order of magnitude $\left(10^{16}\right.$ atoms $)$. On the other hand, the amount of residual oxygen present under high vacuum is a factor 1000 lower than both low vacuum and nitrogen. These results are qualitatively in agreement with the observed experimental differences: both nitrogen and low-vacuum annealing lead to the formation of magnetite, whereas high vacuum annealing only led to the formation of minute amounts of magnetite. However, the number of residual oxygen atoms by itself is not a very insightful quantity for quantitative interpretation.

In analogy to the calculations performed in chapter 4.7.2, the amount of excess iron that could theoretically be extracted from a sample by oxidation with a given amount of residual oxygen and furthermore the thickness of the resulting iron oxide layer can be estimated based on a fixed sample volume. For the calculations, the following 
assumptions are made:

(1) Typical sample dimensions of $1000 \mu \mathrm{m} \times 1000 \mu \mathrm{m} \times 100 \mu \mathrm{m}$.

(2) Formation of magnetite only (based on the Raman analysis of the low vacuum and nitrogen annealed sample above in figure 6.2).

(3) All oxygen present in the furnace reacts with the iron.

The latter is of course an upper estimate, given that the likelihood of oxygen diffusing to the surface of the sample to react with iron continuously decreases the lower the amount of remaining oxygen gets. Furthermore, the iron oxide layer will eventually act as a blocking layer reducing any further oxidation. Therefore, the results presented in table 6.3 are estimates of the maximum amount of excess iron that could theoretically be extracted and the resulting maximum iron oxide layer thickness.

Table 6.3: Amount of iron that can theoretically be removed from the sample via the formation of magnetite and the related magnetite surface layer thickness for nitrogen, low-, and high vacuum annealing.

\begin{tabular}{r|c|c} 
Atmosphere & Excess iron & Surface layer thickness \\
\hline Nitrogen $\left(10^{3} \mathrm{hPa}\right)$ & $3.0 \%$ & $839 \mathrm{~nm}$ \\
Low-vacuum $\left(10^{-2} \mathrm{hPa}\right)$ & $1.2 \%$ & $348 \mathrm{~nm}$ \\
High-vacuum $\left(10^{-5} \mathrm{hPa}\right)$ & $0.001 \%$ & $0.35 \mathrm{~nm}$
\end{tabular}

Under these assumptions the estimated magnetite film thicknesses are, $840 \mathrm{~nm}(3.0 \%$ excess Fe reduction ) for nitrogen, $350 \mathrm{~nm}$ (1.2\% excess Fe reduction) for low vacuum, and $0.35 \mathrm{~nm}(0.001 \%$ excess Fe reduction) for high vacuum (see table 6.3). This simple calculation shows that nitrogen and low vacuum annealing contain sufficient amounts of residual oxygen to remove more than $1 \%$ of interstitial excess iron from the sample. On the other hand, the amount of residual oxygen present during high vacuum annealing is so minute that only $0.001 \%$ of iron can be removed.

Overall, these results agree well with the Raman analysis presented in figure 6.2 . Nitrogen and low vacuum annealing lead to the formation of a substantial iron oxide layer on the surface of the sample. In contrast, virtually no iron oxide was formed during high vacuum annealing. Additionally, the results are consistent with the 
notion that the reported improvements in the superconducting properties after annealing in supposedly 'inert atmospheres', such as nitrogen and low vacuum, are in fact due to the presence of residual oxygen during annealing.

\subsection{Conclusion}

In conclusion, the analysis of air, nitrogen, low- and high vacuum annealed samples indicates that the improvement in the superconducting properties is directly related to the formation of iron oxides, which reduces the amount of interstitial excess iron within the samples and thereby improves the superconducting properties. Consequently, improvements in the superconducting properties are only expected after annealing in an iron-reactant containing atmosphere. Theoretical estimates of the amount of residual oxygen in nitrogen, low vacuum, and high vacuum annealing atmospheres align with the oxidation of the surface as characterised by Raman spectroscopy. Overall, the results of this chapter suggest that the reported improvements in the superconducting properties after annealing in vacuum and nitrogen in the literature are due to the presence of residual oxygen during annealing and not due to increased homogeneity after annealing at elevated temperatures. 


\section{Asymmetric magnetic hysteresis loops in $\mathrm{FeSe}_{1-x} \mathrm{Te}_{x}$}

$\mathrm{FeSe}_{1-x} \mathrm{Te}_{x}$ single crystals typically exhibit symmetric magnetic hysteresis loops (MHLs), that is the magnitude of the magnetisation when increasing the magnetic field, $M_{\text {inc }}(B)$, and decreasing the field, $M_{\text {dec }}(B)$, is approximately equal, leading to a symmetric MHL with respect to $M=0$. This is, to the best of my knowledge, the case for all MHLs of $\mathrm{FeSe}_{1-x} \mathrm{Te}_{x}$ single crystals reported in the literature [Bon12, Das11, Zho14, Sun19] and the majority of the samples studied in this project (see also chapter 5 and 6). However, some of the investigated samples exhibit a pronounced asymmetric hysteresis loop with respect to the $M=0$ axis. Specifically, whilst $M_{\text {inc }}$ still exhibits a typical magnetisation profile, $M_{\text {dec }}$ is close to zero. The observation of such asymmetry is of special interest as it suggests abnormal pinning behaviour that is rarely reported in the iron-based superconductors [Cha09, Zhu12a, Zhu13, Nou21].

In the first part of this chapter, MHLs of four different samples that display this atypical, asymmetric behaviour are presented and analysed in terms of symmetry between positive and negative applied fields, and general reproducibility to exclude potential external disturbances as the origin of this asymmetric behaviour. Thereafter, the relaxation behaviour of one of the samples is studied using time-dependent magnetisation and sweep-rate-dependent MHL measurements. The asymmetric MHL and relaxation behaviour is then explained based on a proposed pinning model that combines both bulk- and surface pinning. Lastly, the results presented here are put in context with several reports on asymmetric MHLs in cuprate superconductors and polycrystalline iron-based superconductors. 


\subsection{Asymmetric magnetic hysteresis loops}

Figure 7.1 shows normalised, asymmetric MHLs of four different $\mathrm{FeSe}_{1-x} \mathrm{Te}_{x}$ samples measured at $2.2 \mathrm{~K}$ (DU14A: air-annealed $\mathrm{FeSe}_{0.15} \mathrm{Te}_{0.85}$, DU23AA: twice air-annealed $\mathrm{FeSe}_{0.35} \mathrm{Te}_{0.65}$, DU49N: nitrogen-annealed $\mathrm{FeSe}_{0.35} \mathrm{Te}_{0.65}, \mathrm{DU}$ 95A: airannealed $\left.\mathrm{FeSe}_{0.35} \mathrm{Te}_{0.65}\right)$. These samples are of different doping, have been annealed in different atmospheres, and are of different size, but still display very similar MHLs. Not only is the field dependence of all four samples almost identical, they also exhibit the same suppression of the magnetisation for the branches with decreasing field, $M_{\mathrm{dec}}$, at both positive and negative applied fields. More specifically, when the applied field is reduced from the maximum value around $\pm 9 \mathrm{~T}$ back to $0 \mathrm{~T}$, the magnetisation drops to almost zero until the applied field is reduced to about $|B|<0.2 \mathrm{~T}$. At this point, $\left|M_{\mathrm{dec}}\right|$ increases rapidly to a value similar to that of the increasing field branch. This behaviour is totally unexpected and has, to the best of my knowledge, only been reported in polycrystalline [Cha09, Zhu12a, Zhu13, Nou21] iron-based superconductors.

Before analysing this behaviour in greater detail, it is worth checking if this behaviour might not be the physical nature of the sample but rather a measurement artefact. Such measurement artefacts could be caused by external distortions or malfunctions of the measurement equipment. A very simple distortion for instance would be the unwanted movement of the sample during the measurement. As described in experimental section 3.3, the working principle of a VSM revolves around the vibration, that is fast upward and downward movement, of the sample. This intended movement can potentially lead to additional, unwanted movement of the sample due to the inertia of sample during the continuous vibration. In the worstcase scenario, unwanted movement could move the sample away from the centred position significantly decreasing the measured magnetisation. If the measurement would have been restricted to positive fields only, one could have argued that the sample might have jumped out of the centred position when the applied field was 


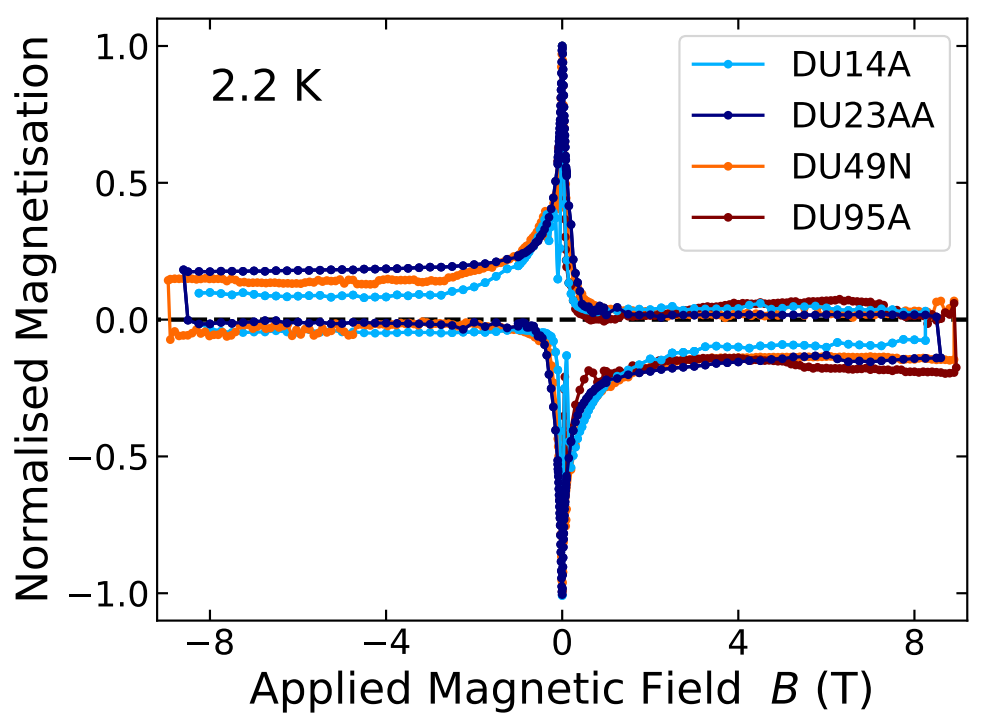

Figure 7.1: Normalised magnetisation versus applied magnetic field, $B$, for four different samples (DU14A: air-annealed $\mathrm{FeSe}_{0.15} \mathrm{Te}_{0.85}$, DU23AA: twice air-annealed $\mathrm{FeSe}_{0.35} \mathrm{Te}_{0.65}$, DU49N: nitrogen-annealed $\mathrm{FeSe}_{0.35} \mathrm{Te}_{0.65}$, DU95A: air-annealed $\mathrm{FeSe}_{0.35} \mathrm{Te}_{0.65}$ ). For ease of comparison of the different samples, the hysteresis loops of each sample have been normalised to the maximum absolute magnetisation of their respective MHL.

switched from increasing to decreasing field and that this is what truly led to the low magnetisation for decreasing fields. However, the fact, that the asymmetry between decreasing and increasing field is symmetric for both positive and negative applied fields excludes this narrative as a potential explanation as the sample would have to jump back and forth between the centred position and out of centre at the exact time of the field switch, which seems very unlikely.

In general, external distortion or malfunctions are typically characterised by shorttime, periodic, or continuous distortions. In the case of external distortions, these could be short, random, one-time distortions; short distortions occurring at a certain frequency like for instance $1 \mathrm{~Hz}$ cryocooler vibrations or $50 \mathrm{~Hz}$ grid noise; or continuous distortions such as large, parasitic, external magnetic fields that are not fully shielded from the sample. Because of this, it is sensible to look at the symmetry of the measurement for positive and negative applied fields more carefully. If distortions were to cause the asymmetric hysteresis loop, one would expect them to manifest in differences between the positive and negative applied field part of the MHL. 
(a)

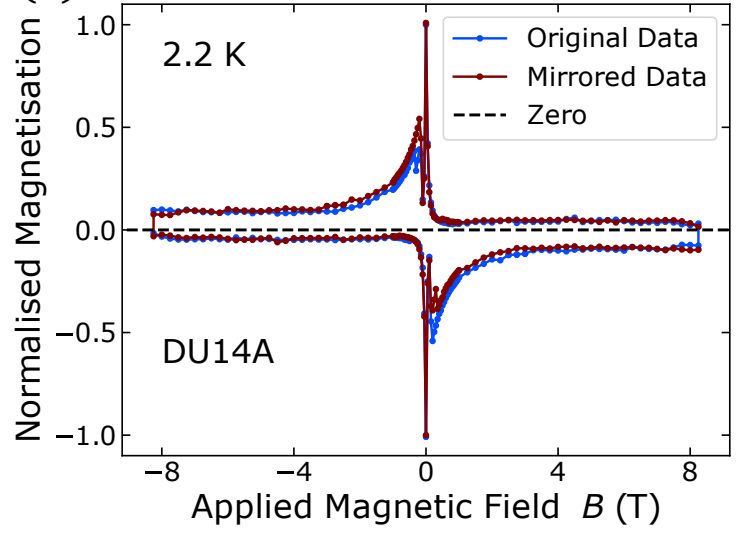

(b)

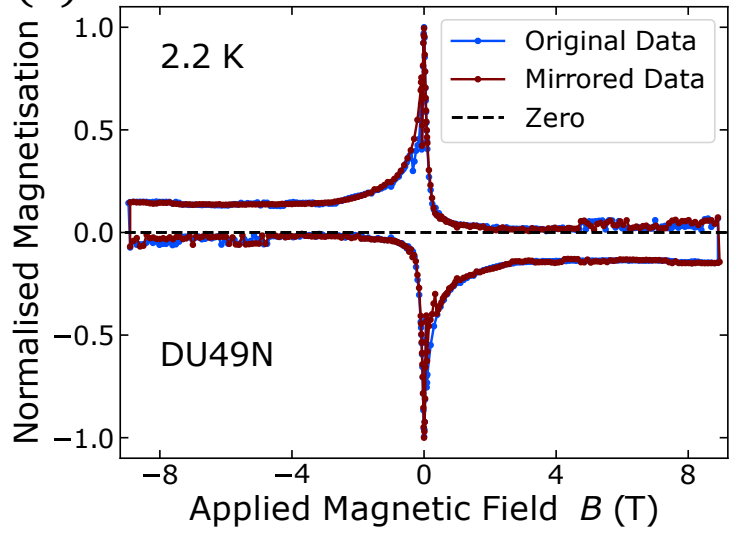

Figure 7.2: MHL and mirrored MHL $(B \rightarrow-B$ and $M \rightarrow-M)$ of DU014A (a) and DU49N (b) at $2.2 \mathrm{~K}$.

For this purpose, the MHL of DU14A (a) and DU49N (b) have been replotted in figure 7.2 ( blue curves) together with their mirrored data $(B \rightarrow-B$ and $M \rightarrow-M$, brown curves). It can immediately be seen that the original MHL of both samples almost perfectly matches their mirrored MHL. This becomes especially clear when comparing the distinct noise of $M_{\mathrm{dec}}$ that develops at fields above about $5 \mathrm{~T}$ in the case of the MHL of DU49N (see figure $7.2(\mathrm{~b})$ ). The noise pattern is almost identical and starts at the exact field for both positive and negative fields. Such behaviour cannot be coincidentally be caused by external distortions, especially given the MHL is measured over more than 7 hours. The comparison of the symmetry hence strongly reinforces the notion that the asymmetric magnetisation for decreasing and increasing field is not an artefact of the measurement but rather an intrinsic property of the samples.

Another key indicator for a correct measurement is reproducibility. As shown in figure 7.3, the MHLs of DU49N measured at four different days with almost a month between the first and last measurement still display the same asymmetry in all four measurements. This reproducibility reinforces that the observed MHL is in fact determined by the physical properties of the sample. Additionally, the fact that the four samples with asymmetric MHLs were measured years apart, when hundreds of other samples measured the same way in between did not exhibit the asymmetric 


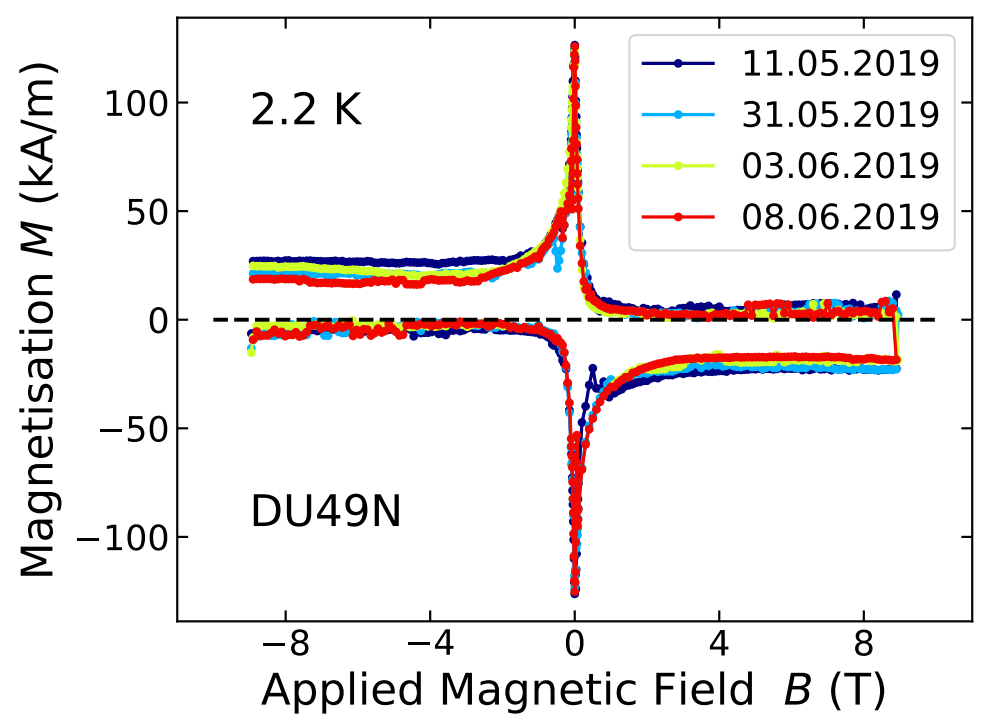

Figure 7.3: MHLs of DU049N at $2.2 \mathrm{~K}$ measured on four different dates.

MHL, again supports this argument.

\subsection{Instability of the asymmetric MHL state}

As mentioned before, asymmetric MHLs were only observed in four out of more than 100 measured $\mathrm{FeSe}_{1-x} \mathrm{Te}_{x}$ samples. Furthermore, trying to purposefully reproduce the asymmetric behaviour in other samples by preparing samples of similar width, thickness, and length combined with the same annealing procedure, has not been met with success. This insinuates that the asymmetric MHL must be very sensitive to slight variations in the structure and properties of the $\mathrm{FeSe}_{1-x} \mathrm{Te}_{x}$ samples.

This sensibility of the asymmetric state is further exemplified by its instability. Figure 7.4 shows the MHLs of DU23AA and DU49N when first measured (blue) and several months later (brown). The first measurement displays the asymmetric MHL as plotted above, but the measurement months later exhibits an essentially symmetric MHL with $M_{\text {inc }}$ basically unchanged but $M_{\text {dec }}$ now mirroring $M_{\text {inc }}$ and consequently doubling the hysteresis loop width. This behaviour illustrates that the properties necessary for the asymmetric MHL are volatile and very sensitive to any change. In 

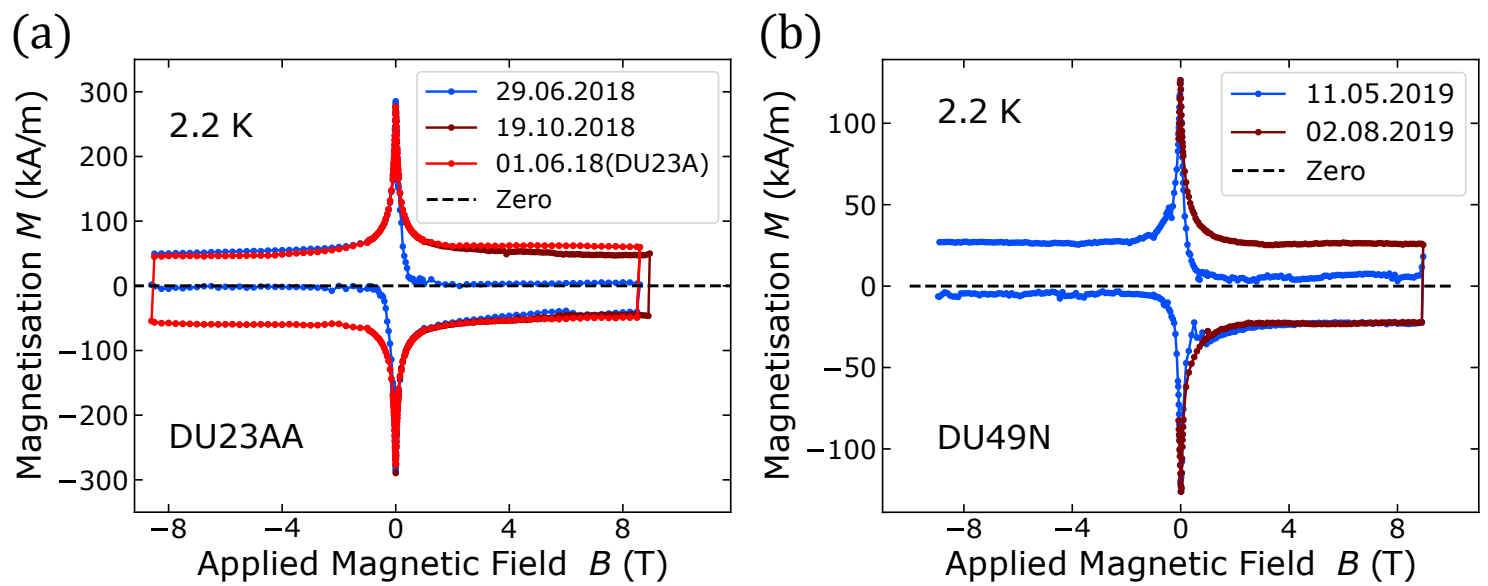

Figure 7.4: MHL of DU23AA (a) and DU49N (b) measured at two different points in time. At first (blue), both sample show highly asymmetric MHLs. Several months later (brown), the asymmetry is lost and both samples exhibit symmetric MHLs with almost twice the width $\Delta M$. Additionally, the MHL of DU23A (same sample before the second air annealing) plotted in red shows similar behaviour to the later MHL (brown) of DU23AA.

this case, further exposure to oxygen and humidity during transportation of the sample and other measurements such as Raman spectroscopy and SEM is likely to have slightly altered the properties of the sample, thus killing the asymmetric behaviour. Additionally, it is also worth pointing out that the asymmetry is not lost because the MHL shifted on the magnetisation axis but actually because the $M_{\mathrm{dec}}$ was largely increased to essentially the same value as $M_{\text {inc }}$, which itself remained unchanged.

To reiterate the sample nomenclature, sample DU23AA has been air-annealed twice with each A representing a separate air annealing step. What is interesting about this sample is the reversibility of the asymmetric hysteresis loop. After the first annealing (figure 7.4(a), red, DU23A), the sample exhibits a large symmetric MHL. This symmetry was initially lost after the second annealing but regained after 3 months of aging. Therefore, the second annealing must have altered the structure or properties of the sample in a way to exactly match what is needed for the asymmetric MHL. However, due to the instability of the asymmetric state, the asymmetry was again lost after a certain time of aging and the sample regained similar properties to that before the second annealing. 


\subsection{Sweep-rate-dependent MHLs}

Because of the high cost of low-temperature magnetisation measurements, measurements are typically performed by quickly sweeping the magnetic field to obtain as much data in a given time as possible. Fast sweeping is further encouraged as the measured magnetisation of a type II superconductor is not only a function of the applied magnetic field, the relative orientation of the sample to the applied field, and the magnetic history, but also the time between a change in the applied field and the measurement of the magnetisation at that field. Specifically, the faster the field is swept, the larger the measured magnetisation [Pus90, Sun92]. Since the measured magnetisation is directly related to $J_{\mathrm{c}}$, the figure of merit that everyone is trying to increase, via Bean's model [Bea62, Bea64], faster sweep rates lead to seemingly larger $J_{\mathrm{c}}$. This is why caution is always warranted when directly comparing the magnitude of $J_{\mathrm{c}}$ obtained from magnetisation measurements without taking the measurement mode into consideration. This is especially true when the measurement mode is not reported, which it always should be [Yes96]. Because of the high cost of low-temperature magnetisation measurements, measurements are typically performed by quickly sweeping the magnetic field to obtain as much data in a given time as possible. Fast sweeping is further encouraged as the measured magnetisation of a type II superconductor is not only a function of the applied magnetic field, the relative orientation of the sample to the applied field, and the magnetic history, but also the time between a change in the applied field and the measurement of the magnetisation at that field. Specifically, the faster the field is swept, the larger the measured magnetisation Pus90, Sun92]. Since the measured magnetisation is directly related to $J_{\mathrm{c}}$, the figure of merit that everyone is trying to increase, via Bean's model [Bea62, Bea64], faster sweep rates lead to seemingly larger $J_{\mathrm{c}}$. This is why caution is always warranted when directly comparing the magnitude of $J_{\mathrm{c}}$ obtained from magnetisation measurements without taking the measurement mode into consideration. This is especially true when the measurement mode is not reported, which it always should be [Yes96]. 
From a research point of view, this effect is of smaller concern as long as it only leads to some variance in the magnitude of the reported $J_{\mathrm{c}}$ depending on the different sweep rates and the relaxation behaviour of the sample. However, as will be shown in this section, fast sweep rates can potentially disguise major properties of a sample that extend further than a simple variation in the reported magnitude of $J_{\mathrm{c}}$. In fact, this might be the main reason why asymmetric MHLs are rarely reported in iron-based superconductor.

Figure 7.5(a) shows MHLs of DU49N measured at four different sweep rates and at persistent fields. The MHL measured at the fastest sweep rate, $150 \mathrm{Oe} / \mathrm{s}$, exhibits the largest MHL at all fields in comparison to the slower sweep rates or the persistent field measurement. Overall, the slower the field was swept, the lower the measured magnetisation. As introduced above and in theory section 2.1.9, this is expected and not particularly special to this sample. However, what is more interesting and special to this sample: The reduction in magnetisation is different for the increasing and decreasing field branch of the loop. At a sweep rate of $150 \mathrm{Oe} / \mathrm{s}$, the magnitude of the magnetisation of the increasing field and decreasing field branch of the MHL are still very similar. With decreasing sweep rate, the difference between the two branches, $\delta M=\left|M_{\text {inc }}\right|-\left|M_{\text {dec }}\right|$, increases as $\left|M_{\text {dec }}\right|$ declines more rapidly than $\left|M_{\text {inc }}\right|$. This is most vividly illustrated by the persistent field measurement where $M_{\mathrm{dec}}$ is close to zero. Furthermore, the noise level of $M_{\mathrm{dec}}$ is also increased in comparison to $M_{\text {inc }}$. Since this increase in noise is not the result of external distortions or increased measurement noise (see section 7.1), it insinuates that the magnetic state of the sample is inherently unstable for $M_{\mathrm{dec}}$. Lastly, it is also worth noting that $M_{\mathrm{inc}}$ exhibits flux jumps at specific applied fields when measured with sweeping field. These jumps are however not present in the persistent measurement and $M_{\mathrm{dec}}$. This suggest, that the pinning determining the magnetisation of $M_{\mathrm{inc}}$ and $M_{\mathrm{dec}}$, and the sweep and persistent field measurements might be of different origin.

To compare the change in the sweep-rate-dependent behaviour after the loss of the asymmetric MHL, the asymmetric MHL measured at 150 Oe/s from (a) is reproduced 

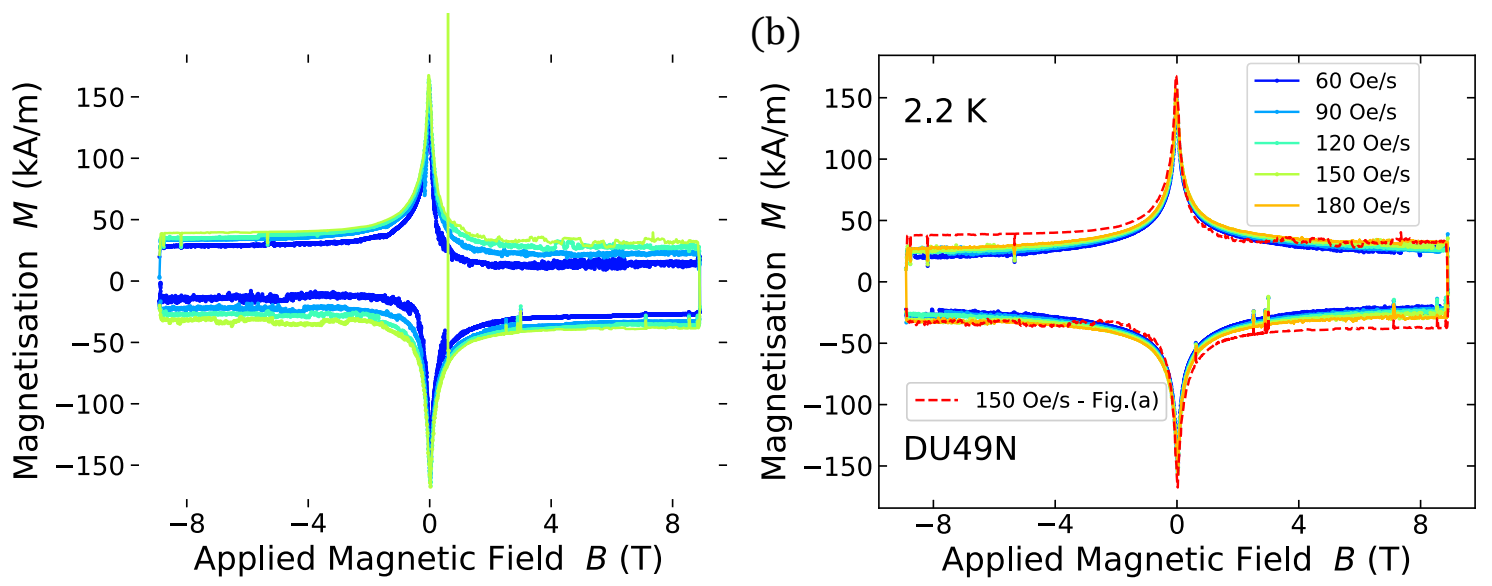

Figure 7.5: Magnetic hysteresis loops of DU049N in the asymmetric state (a) and the symmetric state (b) that was observed in a later measurement (see also figure 7.4(b). The MHLs were measured at different sweep rates at $2.2 \mathrm{~K}$.

in figure 7.5(b) together with the MHLs after the sample has lost the asymmetric behaviour. Again, the magnitude of the magnetisation decreases with reducing sweep rate, but now, the decrease is symmetric for the increasing and decreasing field branches. What is more interesting, the MHL of the fast sweep-rate measurement at the time when the sample showed the asymmetric MHL (red dashed line) is relatively similar to the MHL measured when the asymmetry was lost. In fact, even the flux jumps of $M_{\text {inc }}$ are at the exact same fields (for instance $2.5 \mathrm{~T}, 3.0 \mathrm{~T},-5.3 \mathrm{~T}$, and $-8.2 \mathrm{~T})$. This insinuates that the magnetisation for increasing fields is still largely determined by the same pinning landscape.

\subsection{Time-dependent magnetic relaxation}

Before presenting the data of the time-dependent magnetisation at all measured fields, it is sensible to first analyse the characteristic behaviour as exhibited at three characteristic fields. Figure 7.6 shows 30 -minute time relaxation measurements of the magnetisation of the increasing- and decreasing field branch of the MHL at three characteristic fields: $0.05 \mathrm{~T}, 1 \mathrm{~T}$, and $5 \mathrm{~T}$. These fields have been selected based on the characteristic shape of the hysteresis loop in figure 7.5(a). At $0.05 \mathrm{~T}$, the difference 
between $M_{\text {inc }}$ and $M_{\text {dec }}$ is smallest and the asymmetry is not yet pronounced. At $1 \mathrm{~T}$, the MHL transitions to a more asymmetric behaviour that is fully pronounced at $5 \mathrm{~T}$. The graphs on the left $(a, c, e)$ show the absolute value of the magnetisation and the ones on the right $(b, d, f)$ the magnetisation normalised to the initial magnetisation $M_{0}$ at $t_{0}$, the start of the measurement.

Starting with $\left|M_{\text {inc }}(t)\right|$, the magnetisation curves at $0.05 \mathrm{~T}, 1 \mathrm{~T}$, and $5 \mathrm{~T}$ all exhibit a monotonic decrease in amplitude with time as is expected due to flux creep (see section 2.1.9p. However, the initial decay of the magnetisation is rather slow and does not show a logarithmic time dependence as would be expected based on thermally activated flux creep [And62, And64, Kim62, Yes96]. This initial deviation from the logarithmic time dependence is due to inherent limitations of relaxation measurements. As pointed out by Jirsa et al. [Jir93], the initial measured magnetisation is influenced by the history of the applied magnetic field and is thus not described by flux creep models. Nevertheless, after about $100 \mathrm{~s}$, the influence of the magnetic history diminishes and the magnetisation starts following a logarithmic time dependence. This behaviour is exhibited at all three applied fields.

Like $M_{\mathrm{dec}}$ of the MHLs presented in figure 7.5 , the relaxation of $M_{\mathrm{dec}}$ also exhibits increased noise in comparison to $M_{\text {inc }}$. Still, $M_{\text {dec }}$ at $0.05 \mathrm{~T}$ follows a qualitatively similar magnetic relaxation with time as $M_{\text {inc }}$. At first, the magnetisation decays with a non-logarithmic time dependence, but after about $100 \mathrm{~s}$ the decay again follows a somewhat logarithmic behaviour. At this field, the absolute magnetisation at $t_{0}$, the time at the start of the measurement, is also relatively similar to that of $M_{\text {inc }}: 98 \mathrm{kA} / \mathrm{m}$ versus $120 \mathrm{kA} / \mathrm{m}$. However, over the 30 minutes of relaxation, $M_{\mathrm{dec}}$ decreased by more than $30 \mathrm{kA} / \mathrm{m}(30 \%)$ versus only $15 \mathrm{kA} / \mathrm{m}(12 \%)$ in the case of $M_{\text {inc }}$. This insinuates that the relaxation of $M_{\mathrm{dec}}$ is considerably faster than that of $M_{\text {inc }}$.

The next plotted applied field of $1 \mathrm{~T}$ is just at the transition from the almost symmetric low-field behaviour to the strongly asymmetric behaviour. At this field, $M_{\text {inc }}$ still displays logarithmic flux creep similar to that at $0.05 \mathrm{~T}$. On the contrary, $M_{\mathrm{dec}}$ 

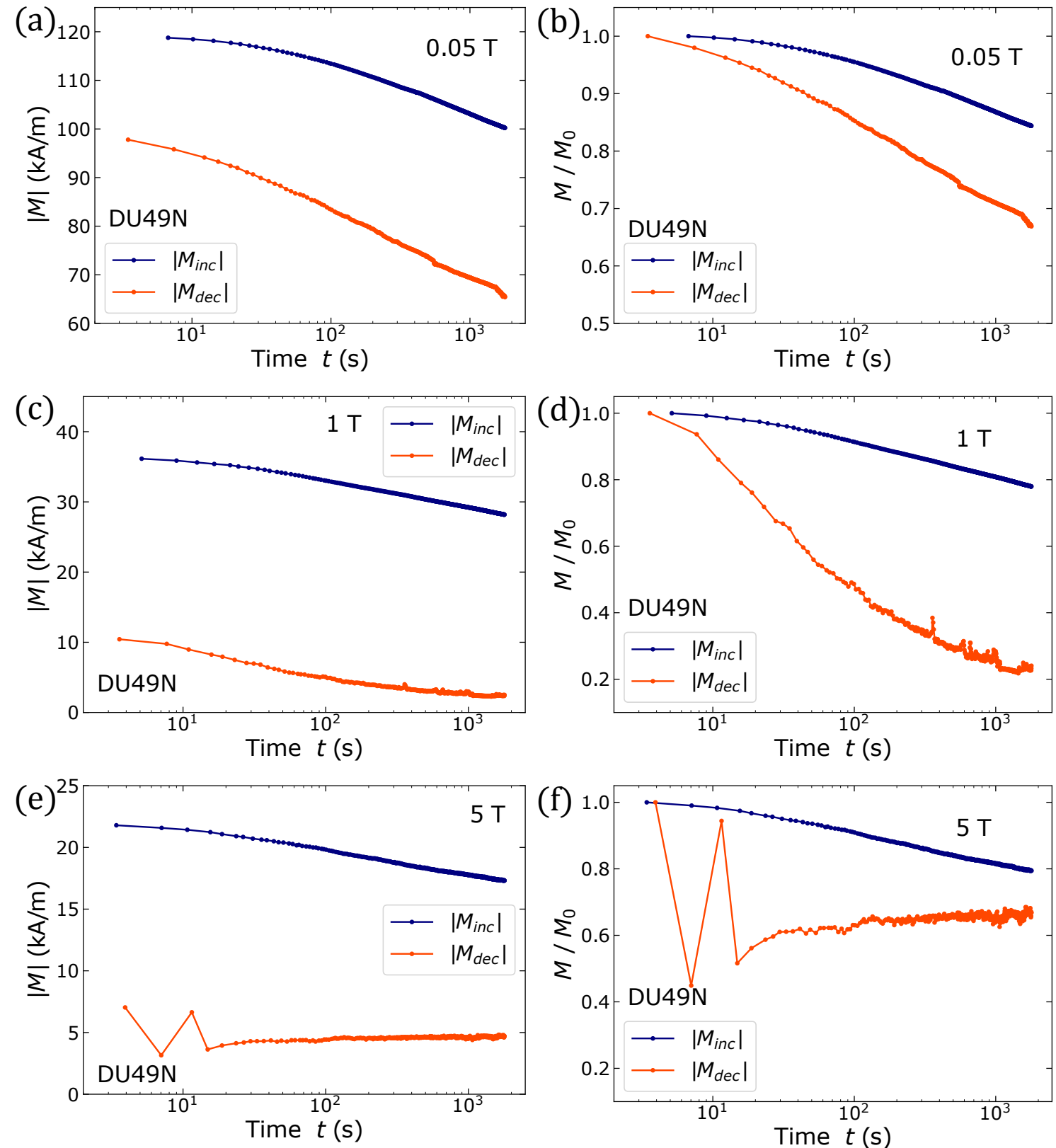

Figure 7.6: Time-dependent magnetisation measurements of DU49N at $2 \mathrm{~K}$ for increasing and decreasing field at $0.05 \mathrm{~T}, 1 \mathrm{~T}$, and $5 \mathrm{~T}$. The graphs on the left $(\mathrm{a}, \mathrm{c}, \mathrm{e})$ show the absolute value of the magnetisation. The graphs on the right (b,d,f) show the normalised magnetisation for ease of comparison of the relative change with time between $\left|M_{\text {inc }}\right|$ and $\left|M_{\mathrm{dec}}\right|$.

does not exhibit a logarithmic decay with time anymore. Instead, as time passes, the relaxation slows down approaching a magnetisation close to zero $(\approx 2.25 \mathrm{kA} / \mathrm{m})$. In other words, the magnetisation approaches a steady state with zero relaxation. 
The remaining moment, $M=2.25 \mathrm{kA} / \mathrm{m}$ is likely due to the parasitic ferromagnetic signal of the iron oxide $\left(M_{\mathrm{dec}}(20 \mathrm{~K}, 1 \mathrm{~T})=2.24 \mathrm{kA} / \mathrm{m}\right)$.

This steady state without magnetic relaxation is even more prominent in $M_{\text {dec }}$ at $5 \mathrm{~T}$. At this field, $M_{\mathrm{dec}}$ does not decrease at all but is basically constant at just under $5 \mathrm{kA} / \mathrm{m}$. This behaviour is highly unusual as any irreversible magnetisation of a superconductor is expected to decrease with time due to relaxation processes [Yes96]. Consequently, the measured magnetisation at this point is solely determined by parasitic ferro- and paramagnetic background contributions and the reversible equilibrium magnetisation.

Figure 7.7 shows the whole set of relaxation measurements taken at 11 different fields for both $M_{\text {inc }}$ and $M_{\text {dec }}$ at $2 \mathrm{~K}, 3 \mathrm{~K}$, and $4 \mathrm{~K}$. In analogy to the behaviour of $\left|M_{\text {inc }}(t)\right|$ at $2 \mathrm{~K}$ for the three different applied fields described above, the decay at most fields and temperatures shows an approximately logarithmic time dependence after $100 \mathrm{~s}$. The only exception to this is the low field magnetisation at $4 \mathrm{~K}$ that follows a similar trend as $\left|M_{\mathrm{dec}}(t)\right|$ at those fields. On the contrary, $\left|M_{\mathrm{dec}}(t)\right|$ exhibits unusual behaviour at almost all fields and temperatures. Starting with $M_{\mathrm{dec}}$ at $2 \mathrm{~K}$, only the low field measurements below $1 \mathrm{~T}$ show a remotely considerable logarithmic decay rate. But even then, instead of one constant decay rate, many display a steplike trend with different decay rates. At higher fields, there is no relaxation at all and the magnetisation remains practically constant at a value below $5 \mathrm{kA} / \mathrm{m}$. To reiterate, this constant magnetisation without relaxation is a key indicator for zero irreversible magnetisation: no flux is pinned at the surface or in the bulk of the superconductor. On a closer look, one notices that the magnitude of the remaining constant magnetisation increases with increasing applied field. However, this is due to the parasitic paramagnetic and ferromagnetic background that increases with increasing field and not related to the superconducting properties of the sample.

At $3 \mathrm{~K}$ and $4 \mathrm{~K}$, this behaviour is even more pronounced as the threshold of the applied field with non-zero relaxation decreases with increasing temperature. In the case of $4 \mathrm{~K}$, only the lowest four applied fields exhibit any relaxation at all. Above 

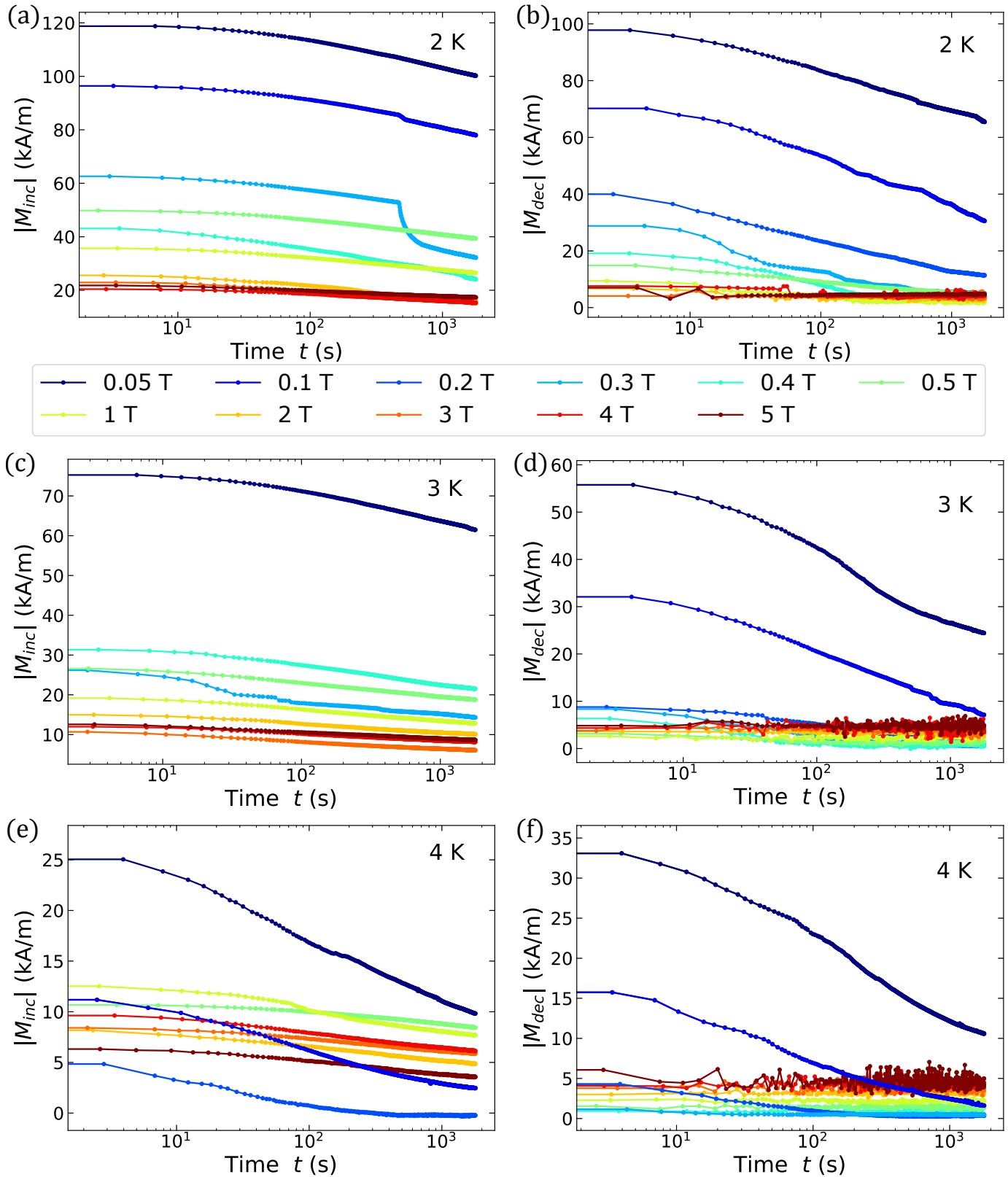

Figure 7.7: Time-dependent magnetisation measurements of DU49N for increasing and decreasing field at $2 \mathrm{~K}, 3 \mathrm{~K}$, and $4 \mathrm{~K}$ at several applied fields. The graphs on the left (a,c,e) show the increasing field magnetisation, $\left|M_{\text {inc }}\right|$. The graphs on the right $(\mathrm{b}, \mathrm{d}, \mathrm{f})$ show the decreasing field magnetisation, $\left|M_{\text {dec }}\right|$. The colour legend between the graphs applies to all graphs.

$0.3 \mathrm{~T}$, the magnetisation remains unchanged over the whole 30 minutes of measurement. The magnitude of the remaining constant magnetisation increases slightly with increasing applied field, which, again, is likely due to the combination of a 
paramagnetic and ferromagnetic background. At first, the magnitude of the background changes quickly with applied field until the ferromagnetic signal is saturated (from $M(0.3 \mathrm{~T})=0.48 \mathrm{kA} / \mathrm{m}$ to $M(2 \mathrm{~T})=3.2 \mathrm{kA} / \mathrm{m})$. At higher fields above $2 \mathrm{~T}$, the background only changes slowly according to a paramagnetic background (from $M(2 \mathrm{~T})=3.2 \mathrm{kA} / \mathrm{m}$ to $M(5 \mathrm{~T})=4.4 \mathrm{kA} / \mathrm{m})$.

To quantitatively analyse the relaxation rate, the magnetisation after $200 \mathrm{~s}$ is fitted to the logarithmic function

$$
|M(t)|=R \ln (t)+M_{0}
$$

where $R$ equals the relaxation rate $\mathrm{d} M / \mathrm{d} \ln (t)$ and $M_{0}$ is the magnetisation offset. Since the parasitic background and the reversible magnetisation do not relaxate [Yes96], only the irreversible magnetisation contributes to the relaxation rate $R$. The results of the fits are shown in figure 7.8 for $\left|M_{\text {inc }}\right|$ and $\left|M_{\text {dec }}\right|$ based on the relaxation measurements of the asymmetric state of the sample (a) from figure 7.7 and for a recent measurement when the sample exhibited symmetric MHLs (b). As mentioned before, some of the measurements exhibit stepwise relaxation. In this case, the data was still fitted as the relaxation rate is still representative of the average relaxation. However, measurements that deviated too much from the logarithmic behaviour have not been fitted.

In general, the temperature- and field-dependent change in the relaxation rate of high $T_{\mathrm{c}}$ superconductor is highly non-trivial and has been extensively researched [Bur92, Das11, Sun14, Wen95, Yes89, Yes96]. However, it is not the main concern of this discussion. Instead, the focus is on the comparison of the increasing- and decreasing field branches of the asymmetric state in comparison to the symmetric state.

Starting with the asymmetric state in figure $7.8(\mathrm{a})$, the relaxation rate is highest at low fields and decreases with increasing field. This is observed for both $\left|M_{\text {inc }}\right|$ and $\left|M_{\text {dec }}\right|$ and at all temperatures. Overall, $R_{\text {inc }}$ and $R_{\text {dec }}$ are of similar magnitude at low 
applied fields. However, as pointed out before, the relaxation of the decreasing field branch comes to a halt at fields above $1 \mathrm{~T}$. Moreover, the applied field at which the relaxation hits zero is dependent on the temperature: the higher the temperature, the lower the field.

Typically, the irreversibility field, $H_{\text {irr }}$ of a superconductor is estimated as the applied field at which the difference between $M_{\mathrm{inc}}$ and $M_{\mathrm{dec}}$ becomes smaller than a certain, chosen threshold. For most iron-based superconductors, this is only feasible at temperatures relatively close to $T_{\mathrm{c}}$ because of the large $H_{\mathrm{irr}}$ that typically exceeds experimental constraints. However, in the case of the asymmetric hysteresis loop and the zero relaxation above a certain temperature-dependent applied field observed here, the irreversibility field can also be estimated from relaxation measurements. Moreover, since the relaxation differs from $M_{\text {inc }}$, which still relaxes, to $M_{\text {dec }}$ with zero relaxation, one can differentiate between $H_{\text {irr,inc }}$ and $H_{\text {irr,dec }}$. $H_{\text {irr,inc }}$ still exceeds the maximum applied field, but $H_{\text {irr,dec }}$ can be estimated for $2 \mathrm{~K}, 3 \mathrm{~K}$, and $4 \mathrm{~K}$ as the field above which the relaxation drops to zero. However, because of the coarse applied field steps, this only leads to a rough estimate: $\left(H_{\text {irr,dec }}(2 \mathrm{~K})<1 \mathrm{~T}\right.$, $\left.H_{\text {irr,dec }}(3 \mathrm{~K})<0.5 \mathrm{~T}, H_{\text {irr,dec }}(4 \mathrm{~K})<0.3 \mathrm{~T}\right)$.

Comparing these results to the symmetric state, one immediately notices that the zero-relaxation behaviour is completely lost. Instead, the increasing and decreasing field branches at both $2 \mathrm{~K}$ and $3 \mathrm{~K}$ are basically identical. Both start at a high relaxation rate at low fields which decreases with increasing field similar to the relaxation behaviour of $M_{\text {inc }}$ in the asymmetric state. The sample has hence lost the atypical relaxation that is connected with the asymmetric MHL. Furthermore, the relaxation rate of the symmetric state is overall lower than that observed in the asymmetric state. 
(a)

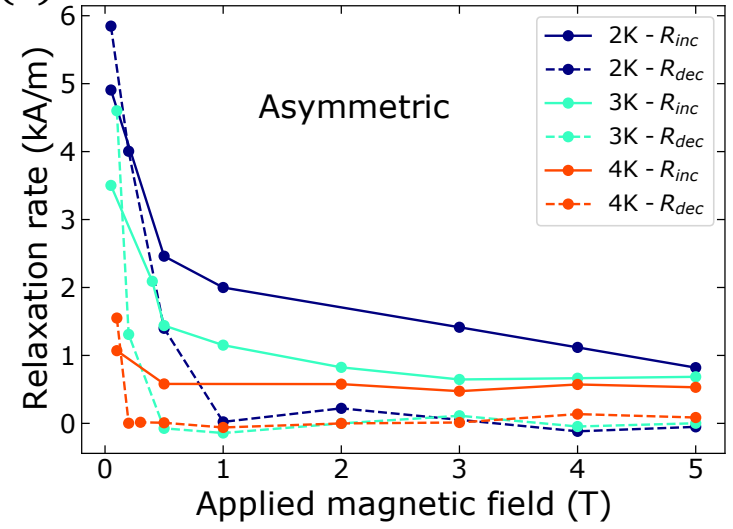

(b)

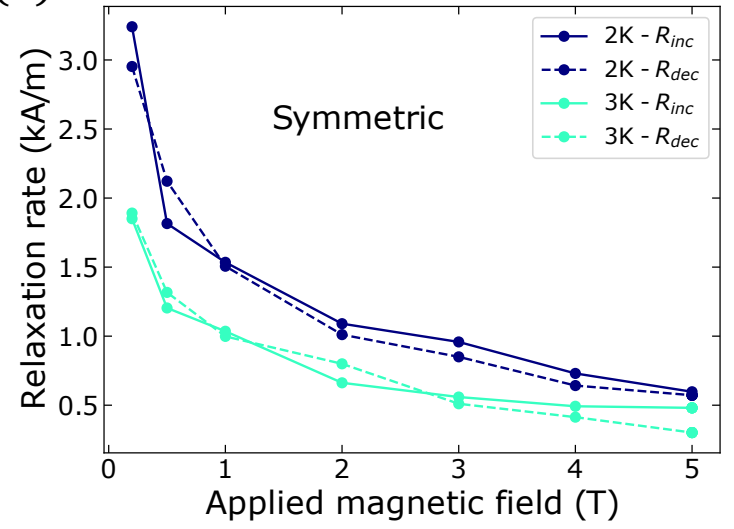

Figure 7.8: Relaxation rate versus applied field for the asymmetric (a) and symmetric state (b) of DU49N.

\subsection{Sweep-rate-dependent magnetic relaxation}

In the previous section, it was shown that the descending magnetisation branch only shows relaxation at low fields. This suggests that there is no irreversible magnetisation for fields above the temperature depend threshold field, $H_{\mathrm{irr}, \mathrm{dec}}$. This is in contrast with the sweep-rate-dependent MHLs in figure 7.5 that show an irreversible magnetisation up to the maximum applied field for both $M_{\mathrm{inc}}$ and $M_{\mathrm{dec}}$. Thus, some kind of relaxation of $M_{\mathrm{dec}}$ must occur on a time scale before the time-dependent magnetic relaxation measurements.

It has been shown by Pust et al. [Pus90, Jir93, Sun92] that the change in magnetisation with sweep rate $\mathrm{d} M / \mathrm{d} \ln (K)$ is directly related to the relaxation rate $\mathrm{d} M / \mathrm{d} \ln (t)$ obtained by time-dependent magnetic-relaxation measurements. This is best illustrated in figure 7.9 that depicts the sweep-rate-dependent MHLs together with the magnetisation from the time relaxation measurements (red vertical lines). The initial measured magnetisation in the relaxation measurements roughly line up with the persistent field MHL. The difference is likely to be a result of the slight difference in temperature at which the two measurements were performed $(2 \mathrm{~K}$ versus $2.2 \mathrm{~K}$ ). From there, the magnetisation of the MHLs from the sweep measurements continuously increases with increasing sweep rate. The analysis of the sweep-rate- 


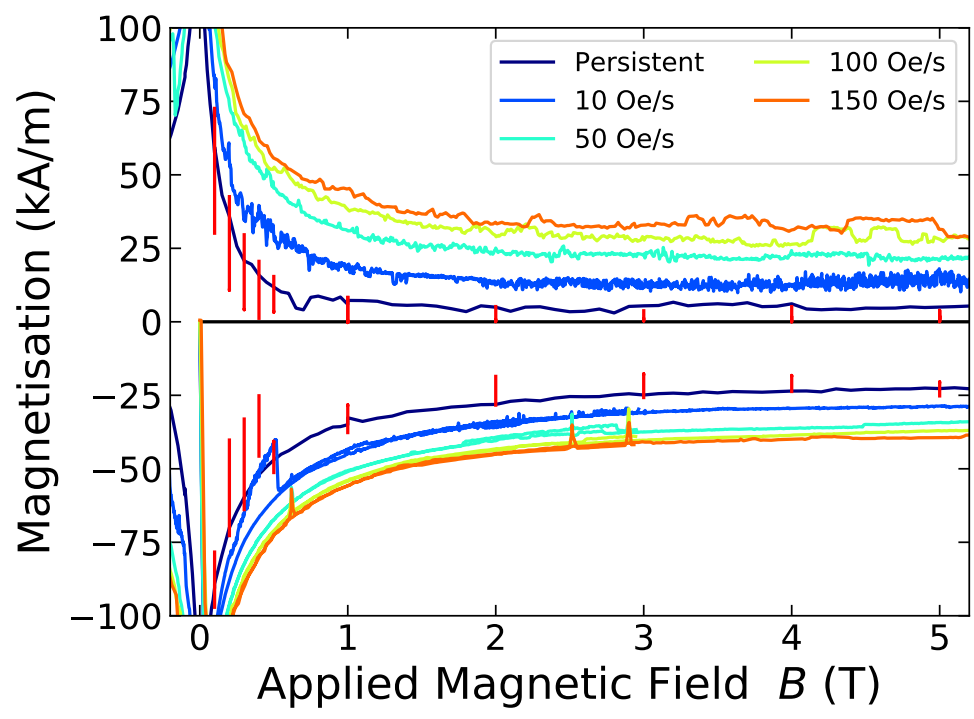

Figure 7.9: MHLs of DU49N at $2.2 \mathrm{~K}$ measured at different sweep rates and persistent field. Additionally, the magnetisation of the time-dependent magnetisation measurement from figure 7.7 are plotted as red vertical lines.

dependent magnetisation therefore allows to extend the relaxation measurements to shorter time scales that are not accessible with classical time relaxation measurements [Pus90, Jir93, Sun92, Yes96].

Based on this connection, the magnetisation obtained in the MHLs is plotted as a function of the logarithm of the sweep rate, $\ln (K)$. Through this, analysis of the magnetic relaxation at a time before the time scale of the time-dependent magnetisation measurements is possible. This is of special interest, since the relaxation of $M_{\text {dec }}$ at higher fields seems to happen before the intrinsic time limit of the relaxation technique. Additionally, since the MHL measurements are not limited to one fixed applied field at a time but are measured for all accessible fields, the analysis can be performed for an almost continuous function of the applied field (depending on the step size of the applied field of the MHL and potential interpolation).

To demonstrate the procedure of the analysis, the case of the relaxation of $\left|M_{\text {inc }}\right|$ and $\left|M_{\mathrm{dec}}\right|$ at applied fields of $4 \mathrm{~T}$ and $-4 \mathrm{~T}$ depicted in figure 7.10 is described in detail. The fields of $4 \mathrm{~T}$ and $-4 \mathrm{~T}$ were chosen for two reasons. First, the asymmetry is largest at fields above $1 \mathrm{~T}$. Secondly and more importantly, the magnetisation in 


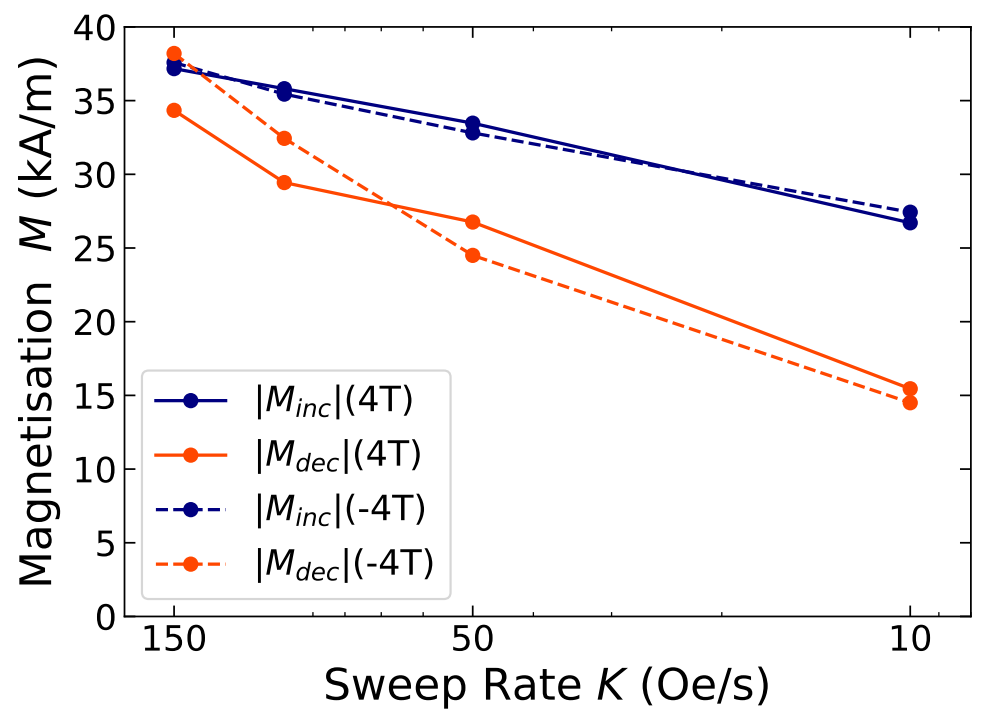

Figure 7.10: Magnetisation of the MHL of DU049N measured at $4 \mathrm{~T}$ and $-4 \mathrm{~T}$ for different sweep rates.

this region is relatively independent of the applied field, which minimises the impact of intrinsic time delays of the measurement equipment [Pus90].

Overall, the magnetisation for increasing and decreasing fields at both positive and negative applied field follow a very similar logarithmic sweep rate dependence, respectively. The slightly larger variance of the two branches of decreasing applied field are simply due to the increased noise in those branches as can be seen in figure 7.5(a). For high sweep rates, $\left|M_{\text {inc }}\right|$ and $\left|M_{\text {dec }}\right|$ have similar amplitudes. However, as the sweep rate decreases, the difference between the two grows larger. At the lowest sweep rate, $\left|M_{\mathrm{dec}}\right|$ is less than half of the initial magnetisation whereas $\left|M_{\mathrm{inc}}\right|$ only decreased by about $25 \%$.

Following the analysis of Pust et al. Pus90] and Sun et al. Sun92] for cuprates, the measured magnetisation can now be fitted as a function of the logarithm of the sweep rate

$$
M(K)=R \ln (K)+C,
$$

where $K$ is the sweep rate, $R$ is the relaxation rate, and $C$ is the magnetisation offset. This fitting procedure has been performed for all applied fields of the MHLs, 
which, for comparison, includes the fields of the time-dependent magnetic relaxation measurements in figure 7.8 .

Figure 7.11(a) shows the relaxation rate $R=|\mathrm{d} M / \mathrm{d} \ln (K)|$ obtained from fitting equation 7.2 to the magnetisation of the different sweep rates at a given field. Firstly, it is worth noting that, as a check for consistency, negative and positive applied fields lead to essentially identical relaxation rates. The relaxation of $M_{\mathrm{inc}}$ exhibits dips in the relaxation due to flux jumps in the MHL but besides that shows consistent relaxation rates. $R_{\text {dec }}$ generally shows a larger variance due to the increased noise in $M_{\text {dec }}$ that also translates into more noise in the fit results.

Comparing the results to the relaxation estimated from the time relaxation measurements, one notes that both $R_{\text {inc }}$ and $R_{\text {dec }}$ are again largest at low fields and decrease with increasing field. The relaxation rate of $M_{\text {inc }}$ follows a similar magnetic field dependence as that of the time relaxation, but the magnitude is overall higher. However, there is one distinct difference between the sweep-rate- and time-dependent measurements: $R_{\text {dec }}$ at higher fields does not drop to zero but instead slowly decreases with field to a finite value of more than 1.5 times that of $R_{\text {inc }}$. Therefore, the sample still exhibits an irreversible magnetisation at this point in time that was not observable in the time relaxation measurements. This vividly demonstrates the benefit of the sweep rate analysis that enables access to a shorter time scale at which the relaxation of the sample can be analysed for both magnetisation branches.

The absolute magnetisation measured at a sweep rate of $150 \mathrm{Oe} / \mathrm{s}$ in figure 7.11 (b) shows that even at high sweep rates there is still a difference between increasing field and decreasing field. Since the magnetisation is background corrected by subtracting the MHL measured at $20 \mathrm{~K}$, the difference here must be the result of an additional contribution in the $M_{\text {inc }}$ branch that is missing in $M_{\text {dec }}$. As will be shown later, this additional contribution is likely due to a surface barrier that leads to an increased magnetisation in the increasing field branch but close to zero in the decreasing branch Cam01. 
(a)

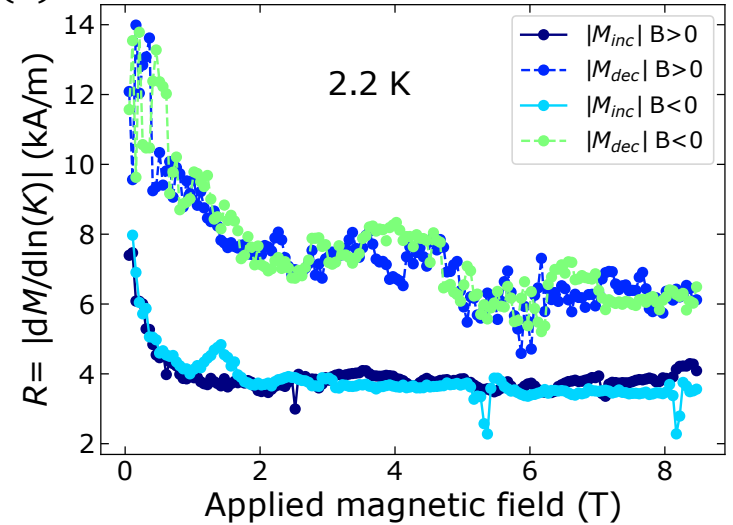

(b)

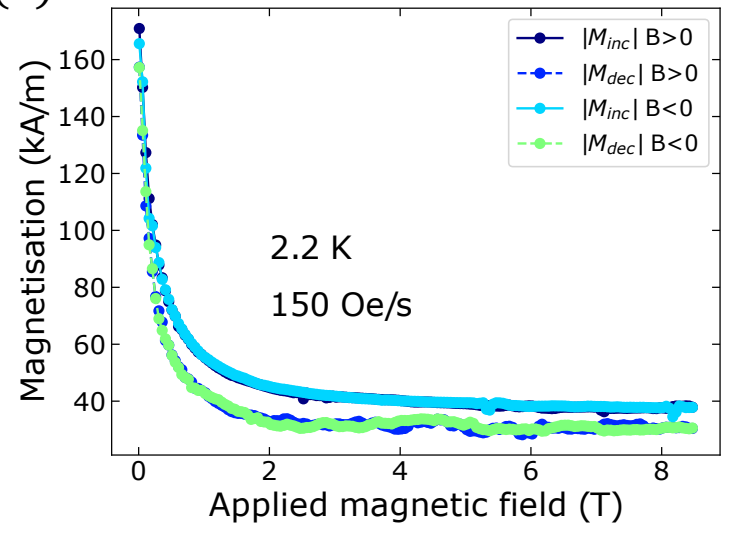

Figure 7.11: (a) Relaxation rate $R=|\mathrm{d} M / \mathrm{d} \ln (K)|$ for $M_{\text {inc }}$ and $M_{\mathrm{dec}}$ at positive and negative applied field. (b) Magnetisation at $150 \mathrm{Oe} / \mathrm{s}$ obtained from the fit function.

As introduced in theory section 2.1.9, it is sensible to not only compare the absolute relaxation rate, $R$, but also the normalised relaxation rate

$$
S=\frac{1}{M} \frac{\mathrm{d} M}{\mathrm{~d} \ln (K)}=\frac{\mathrm{d} \ln (M)}{\mathrm{d} \ln (K)}=\frac{\mathrm{d} \ln (M)}{\mathrm{d} \ln (t)}
$$

which contains information on the actual pinning behaviour of the sample and can be related to the effective barrier height, $U_{\text {eff }}=-k_{\mathrm{B}} T / S$. Figure 7.12 shows $S$ versus applied field for the relaxation obtained from the sweep-rate-dependent MHLs (a) and from the time relaxation rate (b).

Starting with the normalised relaxation rate obtained from the sweep-rate-dependent MHLs at low fields, $S_{\text {dec }}$ is already almost twice as large as $S_{\text {inc }}\left(S_{\text {dec }}(0.1 \mathrm{~T})=0.10\right.$ versus $\left.S_{\text {inc }}(0.1 \mathrm{~T})=0.6\right)$. With increasing applied magnetic field, the relaxation of $M_{\text {inc }}$ steadily increases to about 0.12 at $5 \mathrm{~T}$. On the contrary, $S_{\mathrm{dec}}$ rapidly increases by a factor of three from $=0.1 \mathrm{~T}$ to $B=0.5 \mathrm{~T}$ and remains at an average value around 0.35 for fields up to $5 \mathrm{~T}$. For higher fields, $S_{\text {dec }}$ drops slightly to 0.3 .

In terms of pinning, the effective energy barrier for $M_{\text {dec }}$ at fields above $0.5 \mathrm{~T}$ has to be very small in comparison to that for increasing fields. This difference in the effective barrier height and the resulting relaxation is the origin behind the faster decay of $M_{\text {dec }}$ in comparison to $M_{\text {inc }}$ and ultimately leads to the asymmetric hysteresis loop. 

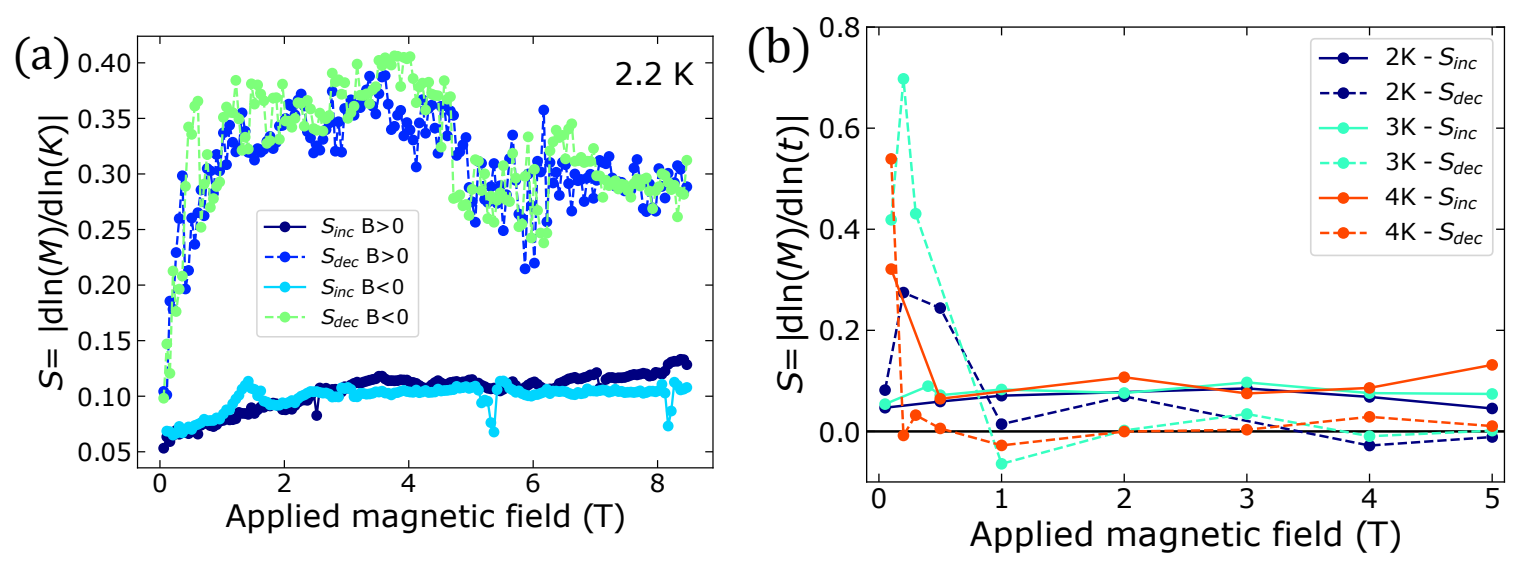

Figure 7.12: (a) Normalised relaxation rate $S$ for $M_{\text {inc }}$ and $M_{\mathrm{dec}}$ at positive and negative applied field obtained from sweep-rate-dependent MHL measurements. (b) Normalised relaxation rate $S$ for $M_{\text {inc }}$ and $M_{\text {dec }}$ obtained from the time-dependent magnetisation measurements.

Furthermore, the slower relaxation of $M_{\text {dec }}$ at low fields aligns with the relatively large magnetisation of $M_{\mathrm{dec}}$ even for persistent field measurements.

Comparing the sweep rate relaxation to the relaxation obtained from the timedependent magnetisation measurements, the differences of the different time scale become very obvious. At short time scales (sweep rate relaxation) and fields above $0.5 \mathrm{~T}, S_{\mathrm{dec}}$ is large and consequently the irreversible magnetisation of $M_{\mathrm{dec}}$ decreases rapidly. As a result, $M_{\mathrm{dec}}(B>0.5 \mathrm{~T})$ has essentially fully decayed at the time of the time-relaxation measurement and the sample exhibits a normalised relaxation rate of effectively zero. Due to the lower short-time relaxation rate at fields below $0.5 \mathrm{~T}$, the sample still exhibits an irreversible magnetisation and a large normalised magnetisation decreasing fields even in the time-dependent measurements.

The normalised relaxation for increasing fields is of finite value for all applied fields. At low fields, the normalised relaxation rate of the two measurements is very similar $\left(S_{\text {inc }}^{\text {time }}(0.05 \mathrm{~T})=0.047\right.$ versus $\left.S_{\text {inc }}^{\text {sweep }}(0.05 \mathrm{~T})=0.054\right)$. With increasing field, $S_{\text {inc }}^{\text {time }}$ also increases similarly to the sweep rate relaxation. However, at fields above $3 \mathrm{~T}$, $S_{\text {inc }}^{\text {time }}$ starts to decrease whereas $S_{\text {inc }}^{\text {sweep }}$ still increases. Overall, the normalised relaxation at both time scales is on the same order of magnitude and the differences are considerably smaller in the case of $S_{\mathrm{inc}}$ in comparison to $S_{\mathrm{dec}}$. As will be presented 
below, this is likely due to the surface pinning that is present at both time scale for $S_{\text {inc }}$, but does not contribute to $S_{\mathrm{dec}}$.

The normalised relaxation rate of similarly doped $\mathrm{FeSe}_{1-x} \mathrm{Te}_{x}$ single crystals has been reported by Das et al. [Das11] and Sun et al. [Sun13c]. Their normalised relaxation rates are on the order of 0.02 to 0.05 , which is on the same order magnitude as the relaxation obtained for increasing field $S_{\text {inc }}$ stated above. However, they are considerably lower than $S_{\text {dec }}^{\text {time }}$ at low fields and $S_{\text {dec }}^{\text {sweep }}$ at fields above $0.5 \mathrm{~T}$ for the sample showing the asymmetric MHL. This reinforces the atypical relaxation of $S_{\text {dec }}$ and consequently a small effective barrier energy.

\subsection{Pinning model for the asymmetric MHL and relaxation}

The analysis of the previous sections has shown that the magnetisation and the related relaxation of the decreasing field branches differs substantially from that of the increasing field branches. Moreover, the difference in the relaxation rate estimated from the time-dependent magnetisation measurements and from the sweeprate MHLs insinuates that there are two regimes that are governed by different pinning behaviours.

In the following, a model based on the interplay of bulk and surface pinning is proposed to explain the observed asymmetric behaviour and the different relaxation regimes. In this model, the total magnetisation is given by the sum of the contribution due to surface pinning and bulk pinning, $M=M_{\text {surf }}+M_{\text {bulk }}$. To avoid unnecessary complication, the bulk pinning is described by the simple Bean model, where the flux gradient is assumed to be independent of the applied field and the surface barrier is assumed to be independent of the applied field and the flux profile inside the superconductor. This model is motivated by the work of Burlachkov [Bur92, Bur93, Kopylov et al. Kop90, Weir [Wei91, Gokhfeld [Gok11, and the potential presence of Bean-Livingston barriers [Bea64a] that are expected to be very pronounced in HTS [Yes96, Kon92]. 
Figure 7.13 shows a schematic of the flux density profile based on the proposed combination of bulk and surface pinning for increasing (a) and decreasing (b) applied field, $B$. As the applied field is increased from zero, the superconductor remains fieldfree until the applied field exceeds $B_{1}$, the effective field for flux entry due to the surface barrier with $B_{1}>B_{\mathrm{c} 1}$ Che93. At $B>B_{1}$, flux starts to penetrate into the sample with increasing applied field according to the Bean model introduced in section 2.1.8. However, the effective field at the boundary of the surface is not equal to the applied field but reduced to an effective field, $B_{\text {eff. }}$ In the case of a field independent surface barrier, the effective field is given by $B_{\text {eff }}=B-B_{1}$. At some field, $B^{*}$, the flux fully penetrates the sample and the maximum bulk magnetisation is obtained (assuming a simple Bean model). Over time, this magnetic state relaxes over both the surface barrier und the bulk of the sample. The rate at which the two relaxation processes take place depends on their respective energy barrier. For the proposed model, it is assumed that the surface barrier dominates over the bulk pinning $\left(U_{\text {surf }}>U_{\text {bulk }}\right)$. This leads to the relaxation profile depicted in figure 7.13(a) at the applied field of $B=B^{*}$ [Bur93]. First, the bulk magnetisation (filled in shades of blue) decreases until a certain crossover time $t^{*}$, at which the bulk magnetisation has completely vanished, is reached. At this point in time, the surface magnetisation (shades of red), which has so far only slightly decreased, equals the total magnetisation of the superconductor. From here, any further relaxation happens over the surface barrier. The time $t^{*}$ in this model is thus representative for the crossover from bulk- and surface pinning to solely surface pinning.

Figure 7.13(b) depicts the changes in the flux density profile as the applied field is decreased from the maximum applied field $B^{*}$ of (a) to $\tilde{B}$. The initial state in (b) at $B^{*}$ before the field is decreased is equal to that of (a) and both the surface and bulk magnetisation contribute to the total magnetisation. Since the surface barrier is assumed to be of Bean-Livingston type, only flux entry is prevented but not flux exit [Bea64a, Kon92]. As a consequence, the initial reduction of the applied field is not changing the effective field seen by the bulk of the superconductor but only 
(b)
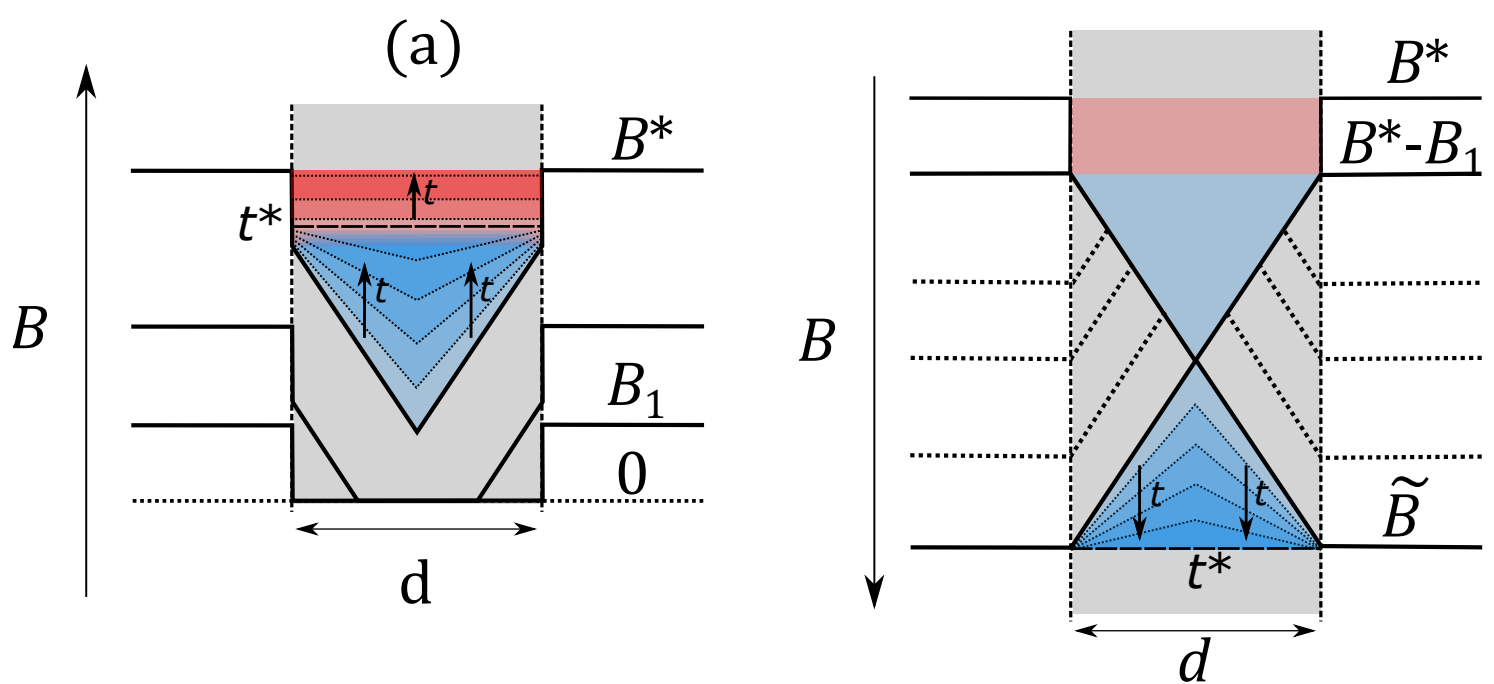

Figure 7.13: Proposed pinning model for a superconducting sample of width $d$ that incorporates both bulk (blue) and surface pinning (red). Figure (a) shows the magnetic flux density for increasing field and (b) for decreasing field.

reduces the surface magnetisation until the field reaches $B=B^{*}-B_{1}$. At this point, the surface magnetisation has vanished and only bulk pinning remains. When the applied field is further reduced, flux starts exiting from the surface region of the sample with the flux in the bulk being trapped as described by the classic Bean model in section 2.1.8. At some applied field, $\tilde{B}$, the flux gradient is fully reversed and the magnetisation is now of opposite polarity. Since there is no surface magnetisation, any relaxation of the magnetisation results from the bulk relaxation (shades of blue in figure $7.13(\mathrm{~b}))$. At $t^{*}$, the bulk pinning has fully relaxed and the sample lost all magnetisation. This is in contrast to (a), where the surface pinning remained at $t \geq t^{*}$ due to the larger barrier energy.

\section{Asymmetric MHLs}

This model can be applied to describe the asymmetric MHL and relaxation behaviour presented above. Starting with the case of the asymmetric hysteresis loop in the persistent field measurements that depends on the direction of the change 
in magnetic field. Persistent field measurements are inherently slow since the field needs to be stabilised at every set point without greatly overshooting. Specifically, the set field is typically approached with a slow field change rate, which inherently leads to relaxation even during the change in field. Consequently, the effective time of the measurement is assumed to be best represented by $t \geq t^{*}$. Following the model of figure 7.13 , the bulk magnetisation has essentially decayed at $t \geq t^{*}$ and the measured magnetisation is almost solely determined by the surface magnetisation. Since the surface barrier is only present for increasing fields, the total magnetisation for decreasing fields is essentially zero. This is exactly what is observed in the asymmetric MHL of the four different samples presented above: a finite magnetisation $M_{\text {inc }}$, but close to zero $M_{\mathrm{dec}}$.

Furthermore, this model also explains the difference between $\left|M_{\text {dec }}\right|$ and $\left|M_{\text {inc }}\right|$ in the case of the fast $150 \mathrm{Oe} / \mathrm{s}$ sweep measurement in figure $7.11(\mathrm{~b})$. Due to the fast sweep rate, both still exhibit bulk pinning and the resulting magnetisation. However, the surface barrier only contributes to the magnetisation of $M_{\text {inc }}$ leading to a slight difference between the two that is on the same order of magnitude as the persistent field $M_{\text {inc }}$.

There is one minor difference, which is not taking into account for in the model. At low fields (e.g. $B<0.2 \mathrm{~T}$ for DU49N), $\left|M_{\text {dec }}\right|$ starts to increase rapidly almost matching $\left|M_{\mathrm{inc}}\right|$. This is a result of the self-field regime, at which the flux density is still relatively small. Thus, even a small amount of pinning centres is able to pin the flux leading to a finite magnetisation. Nevertheless, as the applied field is increased above $0.2 \mathrm{~T}$, the pinning centres are too weak to keep on pinning the increasing amount of flux entering the sample and the effective energy barrier decreases leading to the large relaxation. 


\section{Time- versus sweep-rate-dependent relaxation}

Using this model, the normalised relaxation rate obtained from the time-dependent magnetisation and the sweep-rate-dependent MHLs (see figure 7.12) and their differences can also be explained. To reiterate, the two different techniques both contain information on the pinning behaviour and the relaxation rate, but the time scale at which the two operate differs. In the time-dependent relaxation, the scenario is very much like the persistent field measurement (see initial magnetisation in figure 7.9). The applied field is increased (or decreased) to a certain set field, which is a very slow process. Moreover, the initial magnetic state is not yet in equilibrium but in a state that depends on the exact magnetic history [Yes96. As a result, the logarithmic time dependence only sets in after about $100 \mathrm{~s}$ and thus the obtained relaxation rate is also on that time scale. In terms of the proposed pinning model, this relates to a measurement at a time, $t>t^{*}$. At this time, the bulk magnetisation is fully relaxed and only surface relaxation remains. For increasing fields, this leads to a relatively field-independent normalised relaxation rate. On the contrary, $S_{\mathrm{dec}}$ for fields above 0.5 $\mathrm{T}$ is zero since there is no surface barrier and hence no irreversible magnetisation that would lead to any relaxation. The low field regime differs from this behaviour since very few pinning centres suffice to pin the flux. However, the barrier energy is relative low and the relaxation rate very large at these fields.

Contrary to the time-dependent magnetisation, sweep-rate-dependent MHLs are on a short time scale in terms of relaxation. The field is never stabilised but continuously swept at the given rate: the faster the rate, the shorter the effective time of the measurement [Pus90, Yes96]. In the context of the model, this state would be representative of a state at $t<t^{*}$. Bulk pinning is still present in both the increasing and decreasing branch and thus both exhibit relaxation at all applied fields. For $S_{\text {dec }}$ at fields above $0.5 \mathrm{~T}$, the relaxation is only determined by the bulk relaxation. Since the bulk energy barrier is very low, the normalised relaxation is very fast in that regime (almost ten times as fast as literature values of the same compound 

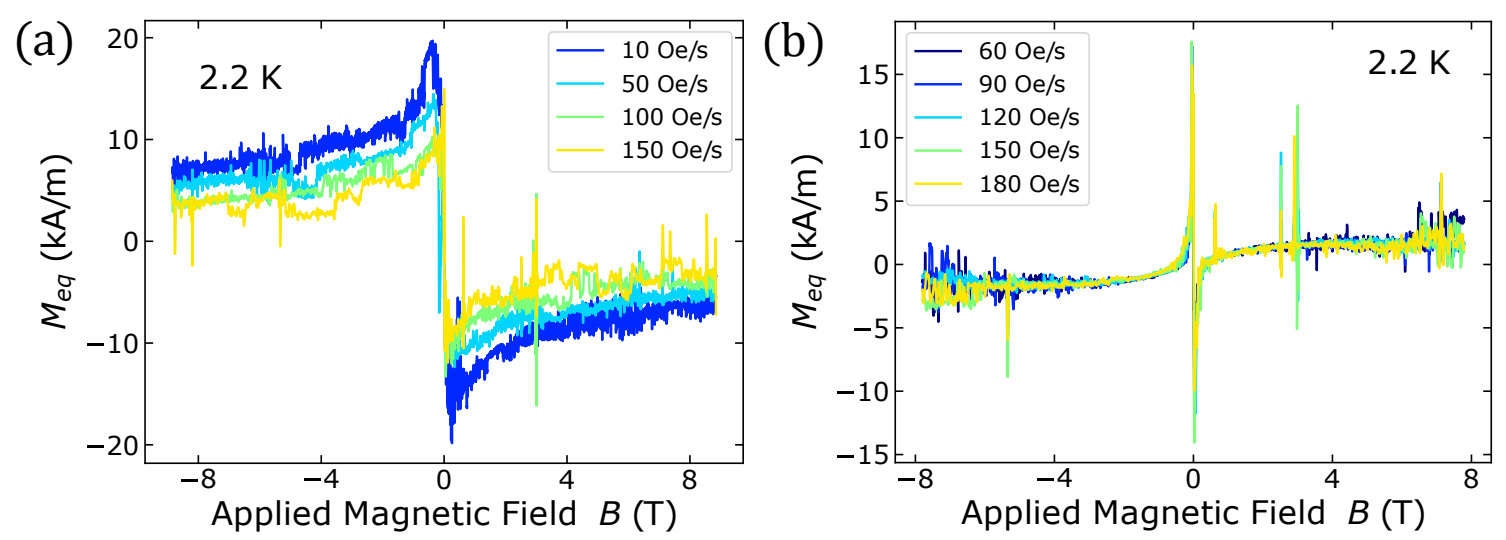

Figure 7.14: Equilibrium magnetisation $M_{\mathrm{eq}}=\left(M_{\mathrm{inc}}+M_{\mathrm{dec}}\right) / 2$ calculated from the MHLs of the asymmetric (a) and symmetric (b) state of DU49N.

[Das11, Sun13c] ). At lower fields, the relaxation is reduced as the pinning centres present suffice to prevent large flux creep at small flux densities. For increasing field, the normalised flux rate is slightly larger than that obtained at $t>t^{*}$. This is due to the combination of both bulk and surface relaxation.

Overall, it is worth noting that the relaxation rate of $S_{\mathrm{inc}}$ is still considerably smaller than that of $S_{\mathrm{dec}}$ even though the model suggests that both should be dominated by fast bulk relaxation. This insinuates that the surface barrier also affects the bulk behaviour of the sample. In one way, this is taken into account for in the model by the reduced effective field experience by the bulk. However, the real interaction between the bulk and surface behaviour is likely to be more complex than what is described by this model. Generally, the model still captures most of the qualitative features observed in the experiments and deviations can be explained based on simplifications and resulting shortcomings of the model.

\section{Critical current density}

Lastly, it is also worth noting that the critical current density cannot be extracted from asymmetric hysteresis loops via the Bean model. As pointed out above, the total magnetisation is given by the sum of the surface and the bulk contribution. In the Bean model, this magnetisation is translated into a flux gradient that is 
proportional to $J_{\mathrm{c}}$. However, this is only correct if the whole magnetisation is a result of this flux gradient. In the case here with a considerable surface barrier, the gradient would be overestimated since the gradient-dependent bulk magnetisation would have to compensate for the surface magnetisation as well. This is best illustrated by the equilibrium magnetisation, $M_{\mathrm{eq}}=\left(M_{\mathrm{inc}}+M_{\mathrm{dec}}\right) / 2$, estimated from the sweeprate dependent MHLs in the asymmetric (a) and symmetric (b) state depicted in figure 7.14. As mentioned before, the equilibrium magnetisation is expected to be independent of the sweep-rate of the MHL measurement [Yes96]. This is the case for $M_{\text {eq }}$ calculated from the symmetric hysteresis loops after the asymmetry was lost (figure 7.14 (b)). However, in the case of the asymmetric hysteresis loops (figure 7.14 (a)), the supposed $M_{\text {eq }}$ is decreasing with increasing sweep-rate. Since $M_{\text {eq }}$ is not sweep-rate-dependent, $M_{\text {eq }}$ must still contain additional contributions and can thus not be correctly estimated from $M_{\mathrm{eq}}=\left(M_{\mathrm{inc}}+M_{\mathrm{dec}}\right) / 2$. In analogy, the simple estimate of the irreversible magnetisation via $M_{\mathrm{irr}}=\left(M_{\mathrm{dec}}-M_{\mathrm{inc}}\right) / 2$, that is used for the estimation of $J_{c}$, is also incorrect in the presence of a pronounced surface barrier Yes96.

\subsection{Asymmetric hysteresis loops in the literature}

The results presented above are to the best of my knowledge the first detailed reports on asymmetric MHLs in single-crystalline iron-based superconductors. However, similar behaviour has occasionally been reported in the cuprate superconductors [Gok11, Kim95, Che93, Che93a, Sen91, Zhu12b] and polycrystalline iron-based superconductors [Zhu12a, Cha09, Nou21]. In the following, the results presented above will be put in context to several such studies that showed similar asymmetric behaviour. 


\subsubsection{Asymmetric MHLs in polycrystalline samples}

In the reports of Senoussi et al. SSen91, Gokhfeld et al. Gok11, Chen et al. Che93, Che93a, and Zhuang et al. [Zhu12a the MHLs taken at low temperatures show symmetric behaviour for increasing and decreasing applied fields. However, as the temperature is increased, the MHLs become increasingly asymmetric. More specifically, the closer the temperatures is to $T_{\mathrm{c}}$, the more pronounced the asymmetry becomes. This behaviour has been explained by all authors by extending the classical Bean model to include the effects of a surface barrier and the equilibrium magnetisation [Che93, Che93a, Sen91, Gok11, Zhu12a. In fact, in the papers of Senoussi [Sen91], Gokhfeld et al. [Gok11], and Chen [Che93, Che93a], they showed that the extended Bean model can be used to calculate theoretical MHLs that match the observed experimental results.

The asymmetric behaviour observed in these reports is very similar to what is observed in the $\mathrm{FeSe}_{1-x} \mathrm{Te}_{x}$ samples described above. This reinforces the suggestion of a surface barrier as the origin of the asymmetry. However, the physical origin of this barrier has to be of different origin since the $\mathrm{FeSe}_{1-x} \mathrm{Te}_{x}$ samples investigated above are not of granular nature but rather single crystalline. Moreover, the asymmetric state is created by annealing and lost in time for the same sample. Even if one were to assume that the annealed samples have become polycrystalline (asymmetric MHL in the annealed state), aging would be very unlikely to induce a transition back to a crystalline state (symmetric MHL in the aged state). Instead, it is more likely that the iron oxide layers, that form on the exposed surfaces of the sample during the annealing process, create barriers between the superconducting regions that act similar to the grain boundaries of the polycrystalline samples. Given the fact that the asymmetry is only observed in a few samples and is also lost after short periods of air-exposure, the nature, most likely the thickness, of the oxide layer has to match certain criteria to act this way.

On another note, the extend of the asymmetry in the reports was shown to depend 
on the temperature at which the MHL is taken. This raises the question if the $\mathrm{FeSe}_{1-x} \mathrm{Te}_{x}$ samples with asymmetric MHLs at $2.2 \mathrm{~K}$ would also show symmetric MHLs at lower temperatures. Given the large difference in $T_{c}$ between $\mathrm{FeSe}_{1-x} \mathrm{Te}_{x}$ and the compounds of the reports, it is sensible to look at similar reduced temperatures, $t=T / T_{\mathrm{c}}$. The reduced temperatures at which the polycrystalline samples of the reports have shown symmetric MHLs are on the order of 0.05. For $\mathrm{FeSe}_{1-x} \mathrm{Te}_{x}$, this would be equal to $0.7 \mathrm{~K}$, which unfortunately is below the lowest measurable temperature of the VSM used in this work.

\subsubsection{Interaction between ferromagnetism and superconductivity}

Zhu et al. [Zhu12b] reported MHLs of YBCO nanoparticles at $5 \mathrm{~K}$ and $300 \mathrm{~K}$. This report is of special interest, because they do not only observe the same asymmetric hysteresis loops in the superconducting state at low temperatures, but also a ferromagnetic signal at room-temperature. This ferromagnetic signal is highly unusual for inherently non-ferromagnetic $\mathrm{YBCO}$ and has been attributed to surface oxygen on the YBCO nanoparticles [Zhu12b]. Overall, their particles are structurally similar to the annealed $\mathrm{FeSe}_{1-x} \mathrm{Te}_{x}$ single crystals: the superconducting bulk is surrounded by a ferromagnetic surface layers. This raises the question if the iron oxide layer might be connected to the pinning behaviour and the origin of the asymmetric MHL. Unfortunately, the author did not dwell on the asymmetry of their MHLs and no mechanism is proposed.

On a side note, it is worth mentioning that their measurements have also been performed on a VSM and, based on the point density, using persistent field measurements. Consequently, their measurements are also expected to be on a similar time scale at an effective measurement time $t>t^{*}$ (see figure 7.13). 


\subsection{Conclusion}

The analysis of this chapter has shown that under certain conditions, annealed $\mathrm{FeSe}_{1-x} \mathrm{Te}_{x}$ single crystals display asymmetric MHLs. These MHLs are not measurement artefacts but intrinsic to the nature of several investigated samples. Furthermore, the asymmetric state was found to be inherently unstable when exposing the sample to atmosphere for too long. The sweep-rate-dependent MHL and relaxation rate analysis has shown that the asymmetry results from weak effective pinning barrier that leads to a fast-decaying magnetisation in the descending field branch. Lastly, a model was proposed to explain the asymmetric MHL and magnetic relaxation behaviour based on the combination of weak bulk pinning and a strong surface barrier. These results were found to agree with earlier reports on cuprate superconductors. 



\section{Conclusion}

In short, this work has shown that the extend of the beneficial effect of annealing on the superconducting properties of $\mathrm{FeSe}_{1-x} \mathrm{Te}_{x}$ depends on the annealing atmosphere, the doping-, the size-, and the micro-structure of the sample. Furthermore, in rare cases, annealing was found to alter the pinning characteristics in a way that induces asymmetric magnetic hysteresis loops.

The structural analysis of as-grown and air-annealed $\mathrm{FeSe}_{1-x} \mathrm{Te}_{x}$ single crystals has shown that air annealing leads to the formation of thin iron oxide layers on the topand bottom surface of the sample. Furthermore, it was shown that inner surfaces can also oxidise if oxygen is able to diffuse into the sample through gap-defects. This oxidation of the inner surfaces is a common if not inevitable occurrence in samples of this compound due to the layered structure. As a result of the formation of iron oxides on the outer- and exposed inner surfaces, the excess iron concentration of the $\mathrm{FeSe}_{1-x} \mathrm{Te}_{x}$ phase is reduced. However, the detailed analysis of the cross section via SEM and STEM as well as depth-profiling XPS analysis revealed that the reduction in the excess iron concentration is not homogeneously distributed in the sample but largest in the region just underneath the oxide layers. This finding suggests that a reduction in the excess iron concentration in the inner parts of the sample must result from the oxidation of the exposed inner surfaces.

In terms of the superconducting properties of differently doped $\mathrm{FeSe}_{1-x} \mathrm{Te}_{x}$ single crystals, air annealing was generally found to have a beneficial effect for all dopings. This improvement of the superconducting properties is directly connected to the formation of the iron oxide layers and the consequential reduction in the excess iron concentration. Ironically, defects that allow the oxidation of inner surfaces are critical for the iron reduction in the inner parts of the sample and the resulting improvement of the superconducting properties in the bulk of the sample. This was 
further illustrated by the study of a stepwise exfoliated, air-annealed sample that showed that the improvement in the superconducting properties after air-annealing is largest in the near surface region, but the superconducting bulk properties are also enhanced compared to the properties of the as-grown crystal.

The summary of doping-dependent superconducting phase diagrams from the literature and our work showed that the critical temperatures of as-grown $\mathrm{FeSe}_{1-x} \mathrm{Te}_{x}$ single crystals vary widely. This is likely to be the result of different synthesis conditions that consequently led to different levels of excess iron. After annealing, the amount of excess iron was reduced to a common level and the critical temperatures of all reports follow a universal superconducting phase diagram. The investigation of annealing in different atmospheres showed that it is the presence of oxygen, or any other iron-reactant, that drives the improvement in the superconducting properties after annealing. In this context, the beneficial effect of supposedly inert atmospheres like nitrogen and low vacuum is connected to the presence of small, but sufficient amounts of residual oxygen during annealing.

The last chapter of this work concerned the rare occurrence of asymmetric hysteresis loops in some of the annealed $\mathrm{FeSe}_{1-x} \mathrm{Te}_{x}$ samples. This asymmetric state was found to be extremely volatile to exposure to air and for this reason was only rarely observed. The origin of this atypical hysteresis loop was analysed using timedependent- and sweep-rate-dependent magnetisation measurements. From this, it was found that the bulk pinning of these samples was very weak and due to flux creep lost very quickly. The irreversible magnetisation that remained during the persistentfield MHL measurements resulted from strong surface pinning. The comparison with similar reports on cuprate superconductors and polycrystalline iron-based superconductors showed that the strong surface pinning is likely to result from the iron oxide layers.

Overall, the analysis presented in this thesis unambiguously connects the improvement of the superconducting properties after annealing to the formation of iron compounds that reduce the amount of interstitial excess iron within the $\mathrm{FeSe}_{1-x} \mathrm{Te}_{x}$ 
phase. In this context, oxygen containing annealing atmospheres were found to be very effective in improving the superconducting properties due to the formation of iron oxides. Furthermore, the reduction in excess iron and improvement in the superconducting properties was shown to be largest just underneath the newly formed iron oxide layer. These results clarify the underlying physics of the annealing process in this relatively young superconductor and enable further in detail studies of the intrinsic properties of $\mathrm{FeSe}_{1-x} \mathrm{Te}_{x}$.

\section{Future work}

The findings on the nature of the annealing process, such as the oxidation of exposed inner surfaces and the largest improvement in the superconducting properties in the region just underneath the oxide layer, motivate further investigation of the annealing procedure to optimise the superconducting properties. More specifically, the study of the thickness- and micro-structure-dependence of the optimal annealing conditions might further reduce the excess iron in the bulk of the sample leading to a larger increase in the overall critical current density. One potential route to improve the annealing process would be repeated annealing and exfoliation steps, where the oxide layer is removed between the annealing steps. This should minimise the amount of excess iron within the sample and thus lead to a maximum improvement in the superconducting properties.

In general, annealed single crystals also enable the study of the effect of other additional post-processing procedures on the superconducting properties of $\mathrm{FeSe}_{1-x} \mathrm{Te}_{x}$ without the suppression of superconductivity due to the high excess iron concentration in the as-grown state. In this context, nano-structured ion implantation of single crystals has been performed towards the end of this thesis. In first preliminary test, a honeycomb structure of gold ions was successfully implanted using a shadow mask and a local ion source. However, the implanted ions are limited to the surface region due to their low energy and no significant change in the superconducting properties 
was observed. In future work, these studies need to be extended by investigating the parameter space in terms of higher implantation energy, variation in fluence, and magnetic and non-magnetic ions.

Lastly, the studies presented in this work were focused on the effect of annealing on $\mathrm{FeSe}_{1-x} \mathrm{Te}_{x}$ crystals. Naturally, the next step is to extend the study to $\mathrm{FeSe}_{1-x} \mathrm{Te}_{x}$ thin films. First attempts to grow undoped FeSe thin films have been made during the course of this thesis using RF-magnetron sputtering. However, even with variation of the growth conditions and substrates, the FeSe films formed in the nonsuperconducting phase and were also relatively brittle. The growth of high-quality FeSe thin films using magnetron sputtering therefore seems relatively challenging. Instead, pulse laser deposition, which is commonly used in the literature, is likely to better suited for the growth of FeSe. 


\section{Publications}

The following lists all peer-reviewed journal articles and conference presentations that have been published or presented during the course of this thesis. The work presented in the first three journal articles is also featured in this thesis.

\section{Peer-reviewed journal articles}

Uhrig D M, Williams G V M, Bioletti G and Chong S V, 2019, Supercond. Sci. Technol., 32(7), 074002 .

Uhrig D M, Williams G V M, Bioletti G and Chong S V, 2020, Supercond. Sci. Technol., 33(5), 055006.

Uhrig D M, Williams G V M, Bioletti G and Chong S V, 2021, IEEE Trans. Appl. Supercond., 31(5), 7300404.

Bioletti G, Williams G V M, Susner M A, Haugen T J, Uhrig D M and Chong S V, 2019, Supercond. Sci. Technol., 32, 064001.

Chong S V, Trompetter W J, Leveneur J, Robinson F, Leuw B, Rumsey B, McCordy M, Turner J, Uhrig D M, Spencer S, Kennedy J and Long N J, 2021, Mater. Sci. Eng. B, 264, 114928. 


\section{Conference presentations}

Uhrig D M, Williams G V M, Bioletti G and Chong S V, International Cryogenic Engineering Conference and International Cryogenic Materials Conference, ICEC/ICMC, September 2018, Oxford, United Kingdom.

Uhrig D M, Williams G V M, Bioletti G and Chong S V, Australian Institute of Physics Congress, AIP, December 2018, Perth, Australia.

Uhrig D M, Williams G V M, Bioletti G and Chong S V, 9th International Conference on Advanced Materials and Nanotechnology, AMN9, February 2019, Wellington, New Zealand.

Uhrig D M, Williams G V M, Bioletti G and Chong S V, New Zealand Institute of Physics, NZIP2019, April 2019, Christchurch, New Zealand.

Uhrig D M, Williams G V M, Bioletti G and Chong S V, European Conference on Applied Superconductivity, EUCAS, September 2019, United Kingdom.

Uhrig D M, Williams G V M, Bioletti G and Chong S V, Applied Superconductivity Conference, ASC2020, November 2020, Tampa (virtual), United States. 


\section{Bibliography}

[Abr57] Abrikosov A A, 1957, J. Exp. Theor. Phys., 5(6), 1174-1182.

[Abr73] Abrikosov A A, 1973, Phys. Today, 26(1), 56-60.

[Ahl69] Ahlborn H, Hornbogen E and Koester U, 1969, J. Mater. Sci., 4, 944-950.

[And62] Anderson P W, 1962, Phys. Rev. Lett., 9(7), 309-311.

[And64] Anderson P W and Kim Y B, 1964, Rev. Mod. Phys., 36(39), 39-43.

[Bao09] Bao W et al., 2009, Phys. Rev. Lett., 102, 247001.

[Bar57] Bardeen J, Cooper N and Schrieffer J R, 1957, Physical Review, 108(5), 1175-1204.

[Bea62] Bean C P, 1962, Phys. Rev. Lett., 8(6), 250-253.

[Bea64] Bean C P, 1964, Rev. Mod. Phys., 36, 31-39.

[Bea64a] Bean C P and Livingston J D, 1964, Phys. Rev. B, 12(1), 14.

[Bea69] Beasley M R, Labusch R and Webb W W, 1969, Phys. Rev., 181(2), 682700.

[Bed86] Bednorz J G and Mueller K A, 1986, Z. Phys. B, 64, 189-193.

[Bla94] Blatter G, Feigelman M V, Geshkenbein V B, Larkin A I and Vinokur V M, 1994, Rev. Mod. Phys., 66(4), 1125-1388.

[Bla04] Blatter G, Geshkenbein V B and Koopmann J A G, 2004, Phys. Rev. Lett., 92(6), 067009.

[Boc1] Dry Air, Gas Code 108, BOC, 27/33 Seaview Road, Seaview, Lower Hutt 5010, New Zealand.

[Boc2] Zero Grade Nitrogen, Gas Code 802, BOC, 27/33 Seaview Road, Seaview, Lower Hutt 5010, New Zealand.

[Bon12] Bonura M, Giannini E, Viennois R and Senatore C, 2012, Phys. Rev. B, 85, 134532. 
[Bra13] Bragg W H and Bragg W L, 1913, Proc. R. Soc. Lond. A, 88, 428-438.

[Bro99] Brown E T, LeMay E, Bursten B E, Murphy C, Woodward P and Stoltzfus M E, 1999, Chemistry: The Central Science, Pearson Education.

[Bur92] Burlachkov L, Yeshurun Y, Konczykowski M and Holtzberg F, 1992, Phys. Rev. B, 45(14), 8193-8196.

[Bur93] Burlachkov L, 1993, Phys. Rev. B, 47(13), 8056-8064.

[Bur96] Burlachkov L, Koshelev A E and Vinokur V M, 1996, Phys. Rev. B, 54(9), 6750-6757.

[Cam91] Campbell A M, Blunt F J, Johnson J D and Freeman P A, 1991, Cryogenics, 31, 732-737.

[Cam01] Campbell A M and Evetts J E, 2001, Adv. Phys., 50(8), 1249-1449.

[Cha05] Chamritski I and Burns G, 2005, J. Phys. Chem. B, 109, 4965-4968.

[Cha09] Chang B C, Hsu C H, Hsu Y Y, Wei Z, Ruan K Q, Li X G and Ku H C , 2009, EPL, 84, 67014 .

[Che93] Chen D X, Cross R W and Sanchez A, 1993, Cryogenics, 33(7), 695-703.

[Che93a] Chen D X, Goldfarb R B, Cross R W and Sanchez A, 1993, Phys. Rev. B, 48(9), 6426-6430.

[Che08] Chen X H, Wu T, Wu G, Liu R H, Chen H and Fang D F, 2008, Nature, 453, 761-762.

[Cle93] Clem J R and Hao Zhidong, 1993, Phys. Rev. B, 48(18), 13774-13783.

[Coo56] Cooper L N, 1956, Phys. Rev., 104(4), 1189-1190.

[Das11] Das P, Thakur A D, Yadav A K, Tomy C V, Lees M R, Balakrishnan G, Ramakrishnan S and Grover A K, 2011, Phys. Rev. B, 84, 214526.

[DeF97] De Faria D L A, Silva S V and De Oliveira M T, 1997, J. Raman Spectrosc., 28, 873-878.

[Deg10] Deguchi K, Mizuguchi Y, Ogawara S, Watanabe T, Tsuda S, Yamaguchi T and Takano Y, 2010, Physica C, 470, 340-341.

[Dew88] Dew-Hughes D, 1988, Cryogenics, 28, 674.

[Dew96] Dewhurst C D, Cardwell D A, Campbell A M, Doyle R A, Balakrishnan G and Paul D McK, 1996, Phys. Rev. B, 53(21), 594-600. 
[Dew01] Dew-Hughes D, 2001, Low Temp. Phys., 27, 713.

[Don11] Dong C, Wang H, Li Z, Chen J, Yuan H Q and Fang M, 2011, Phys. Rev. $B, \mathbf{8 4}, 224506$.

[Don19] Dong L, Zhao H, Zeljkovic I, Wilson S D and Harter J W, 2019, Phys. Rev. Mater, 3, 114801.

[Dur18] Durrell J, Ainslie M D, Zhou D, Vanderbemden P, Bradshaw T, Speller S, Filipenko M and Cardwell D, 2018, Supercond. Sci. Technol., 31, 103501.

[Ens05] Enss C and Hunklinger S, Low-Temperature Physics, Springer, Berlin, Heidelberg, 2005.

[Eis17] Eisterer M, 2017, Supercond. Sci. Technol., 31, 013001.

[Ert79] Ertl G, Huber M and Thiele N, 1979, Z. Nat. forsch., 34a, 30-9, (http: //zfn.mpdl.mpg.de/data/Reihe_A/34/ZNA-1979-34a-0030.pdf).

[Fei89] Feigelman M V, Geshkenbein V B, Larkin A I and Vinokur V M, 1989, Phys. Rev. Lett., 63(20), 2303-2306.

[Fie64] Fietz W A, Beasley M R, Silcox J and Webb W W, 1964, Phys. Rev., 136, A335.

[Fru75] Fruchart D, Convert P, Wolfers P, Madar R, Senateur J P and Fruchart R, 1975, MRS Bull., 10(3), 169-174.

[Fuj99] Fuji T, de Groot F M F, Sawatzky G A, Voogt F C, Hibma T and Okada K, 1999, Phys. Rev. B, 59(4), 3195-3202.

[Gar81] Garbassi F, Bart J C J and Petrini G, 1981, J Electron Spectros Relat Phenomena, 22(2), 95-107.

[Ge14] Ge J-F, Liu Z-L, Liu C, Gao C-L, Qian D, Xue Q-K, Liu Y and Jia J-F, 2014, Nat. Mater., 14, 285-288.

[Ges89] Geshkenbein V,Larkin A, Feigelman A, and Vinokur V, 1989, Physica C, $162,239$.

[Gha16] Gharahcheshmeh M H, Galstyan E, Xu A, Kukunuru J, Katta R, Zhang Y, Majkic G, Li X F and Selvamanickam V, 2017, Supercond. Sci. Technol., 30, 015016.

[Gin50] Ginzburg V L and Landau L D, 1950, Zh. Eksperim. i Teor. Fiz., 20, 1064. 
[Gok11] Gokhfeld D M, Balaev D A, Petrov M I, Poplov S I, Shaykhutdinov K A and Valkov V V, 2011, J. Appl. Phys., 109, 033904.

[Gol18] Goldstein J I, Newbury D E, Michael J R, Ritchie N W M, Scott J H J and Joy D C, 2018, Scanning Electron Microscopy and X-Ray Microanalysis, forth edition, 978-1-4939-6676-9.

[Gra96] Graat P and Somers M A J, 1996, Appl. Surf. Sci, 1OO, 36-40.

[Gra98] Graat P and Somers M A J, 1998, Surf. Interface Anal., 26, 773-782.

[Gri08] Griffiths D J, Introduction to Electrodynamics, Pearson Benjamin Cummings, San Francisco, 2008.

[Hah19] Hahn S et al., 2019, Nature, 570, 496-499.

[Hes89] Hess H F, Robinson R B, Dynes R C, Valles Jr J M and Waszczak J V, 1989, Phys. Rev. Lett., 62(2), 214-216.

[Hik87] Hikami S, Hirai T and Kagoshima S, 1987, Jpn. J. Appl. Phys., 26, L314.

[Hos16] Hossaini S J, Ghorbani S R, Arabi H, Wang X L and Lin C T, 2016, Solid State Commun., 246, 29-32.

[Hsu08] Hsu F C et al., 2008, Proc. Natl. Acad. Sci. U.S.A, 105(38), 14262-14264.

[Hu11] Hu H, Zuo J-M, Wen J, Xu Z, Lin Z, Li Q, Gu G, Park W K and Greene L H, 2011, New J. Phys., 13, 053031.

[Hu12a] Hu J, Wang G C, Qian B and Mao Z Q, 2012, Supercond. Sci. Technol., 25, 084011.

[Hu12b] Hu H et al, 2012, Phys. Rev. B, 85, 064504.

[Hul81] HUL81-Hulm J K, Kunzler J E and Matthias B T, 1981, Phys. Today, 34(1), 34.

[Jir93] Jirsa M, Pust L, Schnack H G and Griessen R, 1993, Physica C, 207, 85-96.

[Jir97] Jirsa M, Pust L, Dlouhy D and Koblischka M R, 1997, Phys. Rev. B, 55(5), 3276-3284.

[Joh95] Johansen T and Bratsberg H, 1995, J. Appl. Phys., 77(8), 3945-3952.

[Joh10] Johnston D C, 2010, Adv. Phys., 59(6), 803-1061.

[Joh15] Johnson P D, Xu G and Yin W-G, Iron-based Superconductivity, Springer Switzerland, 2015. 
[Jos99] Joshi P C and Cole M W, 1999, J. Appl. Phys, 86, 871.

[Jub10] Jubb A M and Allen H C, 2010, ACS Appl. Mater. Interfaces, 2, 2804-2812.

[Kam06] Kamihara Y, Hiramatsu H, Hirano M, Kawamura R, Yanagi H, Kamiya T and Hosono H, 2006, J. Am. Chem. Soc., 128, 10012.

[Kam08] Kamerlingh Onnes H, 1908, KNAW Proceedings, 11, 168-185.

[Kam08a] Kamihara Y, Watanabe T, Hirano M and Hosono H, 2008, J. Am. Chem. Soc., 130, 3296-3297.

[Kam11] Kamerlingh Onnes H, 1911, KNAW Proceedings, 14(2), 818-821.

[Kam13] Kamerlingh Onnes H, 1911, KNAW Proceedings, 15(2), 1406-1430.

[Kam14] Kamerlingh Onnes H, 1914, KNAW Proceedings, 16(2), 987-992.

[Kaw12] Kawasaki Y, Deguchi K, Demura S, Watanabe T, Okazaki H, Ozaki T, Yamaguchi T, Takeya H and Takano Y, 2012, Solid State Commun., 152, 1135-1138.

[Kem16] Kemp S J, Ferguson R M, Khandhar A P and Krishnan K M, 2016, RSC Adv., 6, 77452-77464.

[Kih10] Kihou K et al., 2010, J. Phys. Soc. Japan, 79(12), 124713.

[Kim62] Kim Y B, Hempstead C F and Strnad A R, 1962, Phys. Rev. Lett., 9(7), 306-309.

[Kim63] Kim Y B, Hempstead C F and Strnad A R, 1963, Phys. Rev., 129, 528-535.

[Kim95] Kim Y C, Thompson J R, Christen D K, Sun Y R, Paranthaman M and Specht E D, 1995, Phys. Rev. B, 52(6), 4438-4445.

[Kit08] Kito H, Eisaki H and Iyo A, 2008, J. Phys. Soc. Jpn., 77(6), 063707.

[Kon91] Konczykowski M, Burlachkov L I, Yeshurun Y and Holtzberg F, 1991, Phys. Rev. B, 43(16), 13707-13710.

[Kon92] Konczykowski M, Burlachkov L I, Yeshurun Y and Holtzberg F, 1991, Physica $C, \mathbf{1 9 4}, 155-156$.

[Kop90] Kopylov V N, Koshelev A E and Schegolev I F, 1999, Physica C, 170, 291-297.

[Kos13] Koshika Y, Tomohiro U, Adachi S, Watanabe T, Sakano K, Simayi S and Yoshizawa M, 2013, J. Phys. Soc. Japan, 82, 023703. 
[Kou18] Kouno S et al., 2018, Sci. Rep., 8, 14731.

[Lau14] Laughlin D and Hono K, Physical Metallurgy, Elsevier, 0444537708, 2014.

[Lei10] Lei H, Hu R, Choi E S, Warren J B and Petrovic C, 2010, Phys. Rev. B, 81, 094518.

[Lei16] Lei H et al., 2016, Phys. Rev. Lett., 116, 077002.

[Lim18] Lima M S 1, ElMassalami M, Deguchi K, Takeya H and Takano Y, 2018, J. Phys.: Conf. Ser., 969, 012056.

[Lin15] Lin W, Ganesh P, Gianfranscesco A, Wang J, Berlijn T, Maier T A, Kalinin S V, Sales B C and Pan P, 2015, Phys. Rev. B, 91, 060513.

[Liu09] Liu T J et al., 2009, Phys. Rev. B, 80, 174509.

[Llo87] Lloyd G E, 1987, Mineral. Mag., 51(359), 3-19.

[Lon35] London F and London H, 1935, Proc. R. Soc. Lond. A, 149, 71-88.

[Lut91] Lutz H D and Mueller B, 1991, Phys. Chem. Miner., 18, 265-268.

[Mae88] Maeda H, Tanaka Y, Fukutomi M and Asano T, 1988, Jpn. J. Appl. Phys, 27(2), L209-L210.

[Mah15] Maheshwari P K, Jha R, Gahtori B and Awana V P S, 2015, AIP Advances, 5, 097112.

[Mal05] Malozemoff A P, Mannhart J and Scalapino D, 2005, Physics Today, 58(5), 41-47.

[Med09] Medvedev S et al., 2009, Nat. Mat., 8, 630-630.

[Mei33] Meissner W and Ochsenfeld R, 1933, Naturwissenschaften, 21, 787-788.

[Met87] Metin E and Inal O T, 1987, J. Mater. Sci, 22, 2783-8.

[McQ09] McQueen T M et al., 2009, Phys. Rev. B, 79, 014522.

[Miz08] Mizuguchi Y, Tomioka F, Tsuda S, Yamaguchi T and Takano Y, 2008, Appl. Phys. Lett., 93, 152505.

[Miz10] Mizuguchi Y and Takano Y, 2010, J. Phys. Soc. Jpn., 79, 102001.

[Mom11] Mommma K and Izumi F, 2011, J. Appl. Crystallogr., 44, 1272-1276. 
[Mou92] Moulder J F, Stickle W F, Sobol P E and Bomben K D, Handbook of Xray Photoelectron Spectroscopy, Perkin-Elmer Corporation, Eden Prairie, Minnesota, United States of America, 1992.

[Nel08] Nellist P D and Pennycook S J, 2008, Adv. Imaging Electron Phys., 113(2000), pp 147-203.

[Noj10] Noji T, Suzuki T, Abe H, Adachi T, Kato M and Koike Y, 2010, J. Phys. Soc. Jpn., 8, 084711.

[Nou21] Nouailhetas Q et al., 2021, AIP Advances, 11, 015230.

[Oka91] Okamoto H, 1991, Journal of Phase Equilibria, 12(3), 383-389.

[Oka11] Okazaki K, Sugai S, Niitaka S and Takagi H, 2011, Phys. Rev. B, 83, 035103.

[Oza16] Ozaki T, Wu L, Zhang C, Jaroszynski J, Si W, Zhou J, Zhu Y and Li Q, 2016, Nat. Commun., 7, 13036.

[Oza18] Ozaki T, Wu L, Zhang C, Si W, Jie Q and Li Q, 2018, Supercond. Sci. Technol., 31, 024002.

[Pal15] Pallecchi I, Eisterer M, Malagoli A and Putti M, 2015, Supercond. Sci. Technol., 28, 114005.

[Pip53] Pippard A B, 1953, Proc. Roy. Soc., 216, 547.

[Pra16] Prakash T, Williams G V M, Kennedy J and Rubanov S, 2016, J. Appl. Phys., 120, 123905.

[Pro18] Prozorov R and Kogan V G, Kennedy J and Rubanov S, 2018, Phys. Rev. Appl., 10, 014030.

[Pus90] Pust L, Kadlecova J, Jirsa M and Durcok S, 1990, J. Low Temp. Phys., 78, 179-186.

[Put10] Putti M et al., 2010, Supercond. Sci. Technol., 23, 034003.

[Qua08] Quantum Design08, 2008, VSM Option User Manual, Rev A3.

[Qui62] Quinn D J and Ittner W B, 1962, J. Appl. Phys., 33, 748.

[Ram28] Raman C V and Krishnan K S, 1928, Nature, 121(3048), 501-502.

[Rei14] Reifert D, Hasan N, Doering S, Schmidt S, Monecke M, Feltz M, Schmidl F, Tympel V, Wisniewski W and Moench I, 2014, Supercond. Sci. Technol., 27, 085003. 
[Rod93] Rodriguez-Carvajal J, 1993, Physica B, 192, 55-69.

[Rod11] Rodriguez E E, Stick C, Hsieh P-Y, Butch N P, Paglione J and Green M A, 2011, Chem. Sci., 2, 1782.

[Rot08] Rotter M, Tegel M and Johrendt D, 2008, Phys. Rev. Lett., 101, 107006.

[Sal09] Sales B C, Sefat A S, McGuire M A, Jin R Y and Mandrus D, 2009, Phys. Rev. B, 79, 094521.

[Sen91] Senoussi S, Aguillon C and Manuel P, 1991, Physica C, 175, 202-214.

[Sen92] Senoussi S, 1992, J. Phys. France, 2, 1041-1257.

[Sha99] Shantsev D V, Galperin Y M, Johansen T H, 1999, Phys. Rev. B, 61, 9699.

[She03] Shebanova O N and Lazor P, 2003, J. Solid State Chem., 174, 094521.

[Shi14] Shimoyama J, 2013, Supercond. Sci. Technol., 27, 044002.

[Si13] Si W, Han S J, Shi X, Ehrlich S N, Jaroszynski J, Goyal A and Li Q, 2013, Nat. Commun., 4, 1347.

[Sun92] Sun Y R, Thompson J R, Christen D K, Ossandon J G, Chen Y J and Goyal A, 1992, Phys. Rev. B, 46(13), 8480-8486.

[Sun13] Sun Y, Taen T, Tsuchiya Y, Ding Q, Pyon S, Shi Z and Tamegai T, 2013, Appl. Phys. Express, 6, 043101.

[Sun13a] Sun Y, Tsuchiya Y, Yamada T, Taen T, Pyon S, Shi Z and Tamegai T, 2013, J. Phys. Soc. Japan, 82, 093705.

[Sun13b] Sun Y, Tsuchiya Y, Yamada T, Taen T, Pyon S, Shi Z and Tamegai T, 2013, J. Phys. Soc. Japan, 82, 115002.

[Sun13c] Sun Y, Taen T, Tsuchiya Y, Pyon S, Shi Z and Tamegai T, 2013, EPL, 103, 115002.

[Sun13d] Sun Y, Taen T, Tsuchiya Y, Shi Z and Tamegai T, 2013, Supercond. Sci. Technol., 26, 015015.

[Sun14] Sun Y, Tsuchiya Y, Taen T, Yamada T, Pyon S, Sugimoto A, Ekino T, Shi Z and Tamegai T, 2014, Sci. Rep., 4, 57013.

[Sun16] Sun Y, Yamada T, Pyon S and Tamegai T, 2016, Sci. Rep., 6, 32290.

[Sun19] Sun Y, Shi Z and Tamegai T, 2019, Supercond. Sci. Technol., 32, 103001. 
[Syl18] Sylva G et al., 2018, Supercond. Sci. Technol., 31, 054001.

[Tae09] Taen T, Tsuchiya Y, Nakajima Y and Tamegai T, 2009, Phys. Rev. B, 80, 092502 .

[Tam12] Tamegai T, Toshihiro T, Hidenori Y, Yuji T, Shyam M, Tomotaka T, Yasuyuki N, Satoru O, Masato S and Hisashi K, 2012, Supercond. Sci. Technol., 25, 084008 .

[Tam16] Tamegai T, Sun Y, Yamada T and Pyon S, 2016, IEEE Trans. Appl. Supercond., 26, 7300205.

[Tel12] Telesca D, Nie Y, Budnick J I, Wells B O and Sinkovic B, 2012, Phys. Rev. $B, 85,214517$.

[Tin96] Tinkham M, 1996, Introduction to Superconductivity - Second edition, ISBN13: 978-0-486-43503-9.

[Tsu11] Tsurkan V, Deisenhofer J, Guenther A, Kant Ch, Klemm M, Krug von Nidda H A, Schrettle F and Loidl A, 2011, Eur. Phys. J. B, 79, 289-299.

[Ug119] Uglietti D, 2019, Supercond. Sci. Technol., 32(7), 053001.

[Vie10] Viennois R, Giannini E, Van der Marel D and Cerny R, 2010, J. Solid State Chem., 183, 769.

[Wan08] Wang X C, Liu Q Q, Gao W B, Zang L X, Yu R C, Li F Y and Jin C Q, 2008, Solid State Commun., 148, 538-540.

[Was11] Waseda, Y, Matsubara, E and Shinoda K, 2011, X-ray diffraction crystallography: introduction, examples and solved problems, Springer Science and Business Media.

[Wat07] Watanabe T, Yanagi H, Kamiya T, Kamihara Y, Hiramatsu H, Hirano M and Hosono H, 2007, Inorg. Chem., 46, 7719-7721.

[Wei91] Weir S T, Nellis W J, Dalichaeouch Y, Lee B W, Maple M B, Liu J Z and Shelton R N, 1991, Phys. Rev. B, 43(4), 3034-3041.

[Wen95] Wen H, Schnack H G, Griessen R, Dam B and Rector J, 1995, Physica C, 241, 353-374.

[Wil18] Williams G V M, Prakash T, Kennedy J, Chong S V and Rubanov S, 2018, J. Magn. Magn. Mater, 460, 229-233.

[Wri91] Wriedt H A, 1991, J. Phase Equilibria , 12(2), 170-200. 
[Wu87] Wu M K, Ashburn J R, Torng C J, Hor P H, Meng R L, Gao L, Huang Z J, Wang Y Q and Chu C W, 1987, Phys. Rev. Lett., 58(9), 908-910.

[Xia09] Xia T L et al., 2009, Phys. Rev. B, 79, 140510.

[Xu93] Xu M, Finnemore D K, Crabtree G W, Vinokur V M, Dabrowski B, Hinks D G and Zhang K, 1993, Phys. Rev. B, 48(14), 10630-10633.

[Yad11] Yadav C S and Paulose P L, 2011, Solid State Commun., 151, 216-218.

[Yam16] Yamada T, Sun Y, Pyon S and Tamegai T, 2016, J. Phys. Soc. Japan, 85, 024712.

[Yes88] Yeshurun Y and Malozemoff A P, 1988, Phys. Rev. Lett., 60, 2202.

[Yes89] Yeshurun Y, Malozemoff A P, Worthington T K, Yandrofski R M, KrusinElbaum L, Holtzberg F H, Dinger T R and Chandrashekhar G V, 1989, Cryogenics, 29, 258-262.

[Yes96] Yeshurun Y, Malozemoff A P and Shaulov A, 1996, Rev. Mod. Phys., 68(3).

[Zha06] Zhang L, Xu X B, Qiao Q, Yao X, Wang Q H and Ding S Y, 2006, Supercond. Sci. Technol., 19, 443-446.

[Zha09] Zhang L, Singh D J and Du M H, 2009, Phys. Rev. B, 79, 012506.

[Zha18] Zhang L et al., 2018, Science, 360(6385), 182-186.

[Zha19] Zhang L et al., 2019, Nature Physics, 15, 41-47.

[Zhu12a] Zhuang J C, Sun Y, Ding Y, Yuan F F, Liu J T and Shi Z X, 2012, Mod. Phys. Lett. B, 26(30), 1250197.

[Zhu12b] Zhu Z et al., 2012, Phys. Chem. Chem. Phys., 14, 3859-3863.

[Zhu13] Zhuang J C, Sun Y, Ding Y, Yuan F F, Jia X, Shi Z X and Huang H Y, 2013, J. Low Temp. Phys., 172, 113-121.

[Zho14] Zhou W, Sun Y, Zhang S, Zhuang J, Yuan F, Li X, Shi Z, Yamada T, Tsuchiya Y and Tamega T, 2014, J. Phys. Soc. Japan, 83, 064704. 